This document is downloaded from DR-NTU (https://dr.ntu.edu.sg) Nanyang Technological University, Singapore.

Designed antimicrobial peptides :

structure-activity correlations and mode of action

Harini Mohanram

2013

https://hdl.handle.net/10356/61757

https://doi.org/10.32657/10356/61757 

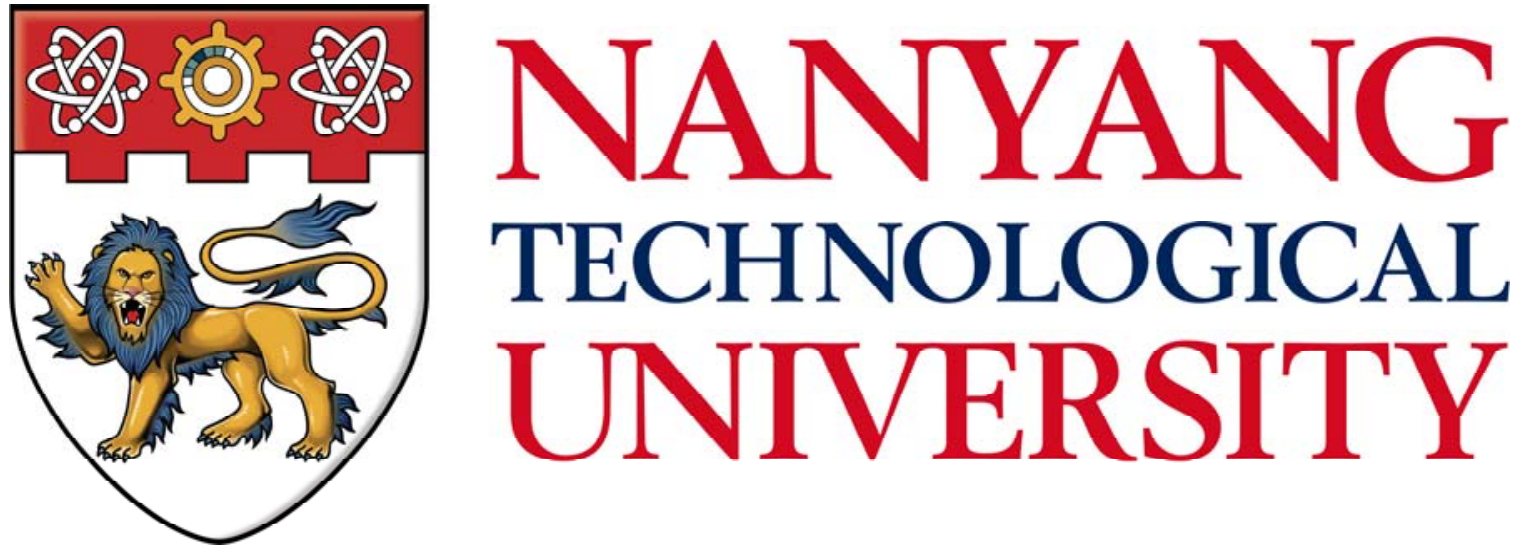

DESIGNED ANTIMICROBIAL PEPTIDES: STRUCTURE-ACTIVITY CORRELATIONS AND MODE OF ACTION

\author{
HARINI MOHANRAM \\ SCHOOL OF BIOLOGICAL SCIENCES \\ 2013
}




\title{
DESIGNED ANTIMICROBIAL PEPTIDES: STRUCTURE-ACTIVITY CORRELATIONS AND MODE OF ACTION
}

\author{
HARINI MOHANRAM
}

SCHOOL OF BIOLOGICAL SCIENCES

A thesis submitted to the Nanyang Technological University in partial fulfillment of the requirement for the degree of Doctor of Philosophy

2013 


\section{DESIGNED ANTIMICROBIAL PEPTIDES: STRUCTURE-ACTIVITY CORRELATIONS AND MODE OF ACTION}

A thesis for the degree of Doctor of Philosophy at Nanyang Technological University

\section{HARINI MOHANRAM}

Supervised by A/P. Surajit Bhattacharjya

School of Biological Sciences

Nanyang Technological University 


\section{ACKNOWLEDGEMENTS}

First of all, I am greatly indebted to my supervisor Associate Professor. Surajit Bhattacharjya for extending his helping hands to provide me a platform to learn and research under his guidance. His incredible patience and meticulous support through out these years has been the driving force in successfully completing my Ph.D. I am also equally grateful to Dr. Anirban Bhunia whose encouragement and supportive words made me to apply for $\mathrm{PhD}$. I take this opportunity to thank him for both online and offline support.

There are no words to explain the patience and support put forth by my husband Mr.Balaji Vignesh Chand and I am also equally indebted to thank my kids, Nivetha and Kirthi, who helped me to smile during most difficult times. I would also like to extend my sincere thanks to my Parents, Parent-in-laws and my extended family for the support and encouragement.

My thanking session would not get completed if I don't thank my friends who have been my pillar of strength. First of all, I would like to thank Rosi for fruitful lunchtime

discussions and provide me emotional support. I would also like to acknowledge all my lab members, Sebanti, Mukesh, GeokLin for the support and a pleasant and joyful working environment.

Lastly, I would also like to thank School of Biological Sciences, NTU for giving me the chance of research and NTU for Scholarship. 


\section{Table of contents}

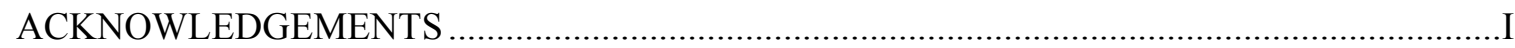

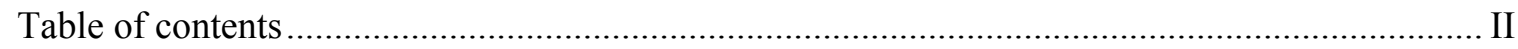

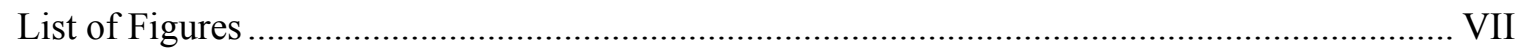

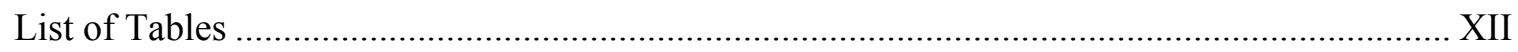

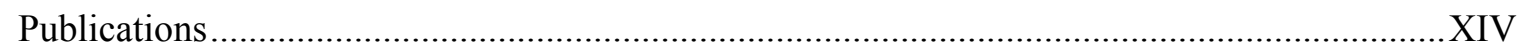

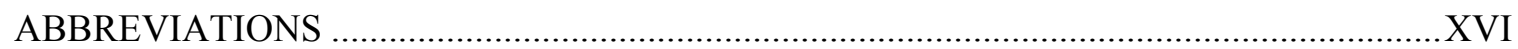

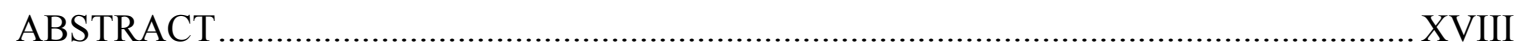

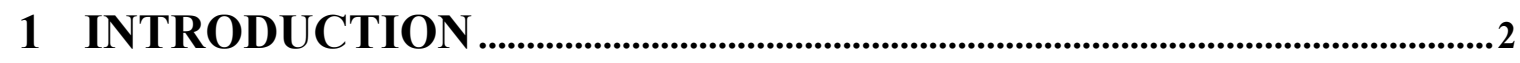

1.1 Antibiotic resistance and emergence of Antimicrobial peptides (AMPs): ..................2

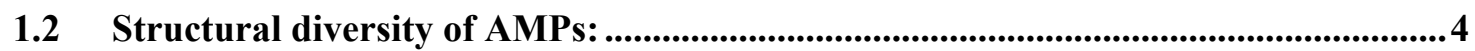

1.2.1 Alpha helical antimicrobial peptides:............................................................. 4

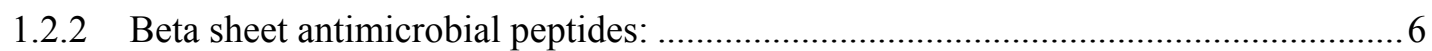

1.2.3 Extended and Loop structures of AMPs: .............................................................. 7

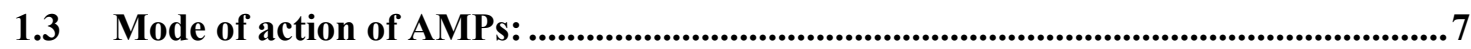

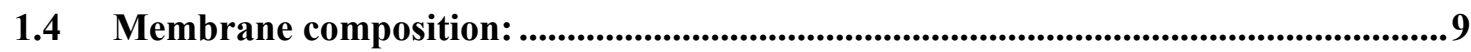

1.5 Membrane lipids and curvature strains - an insight into membrane permeation

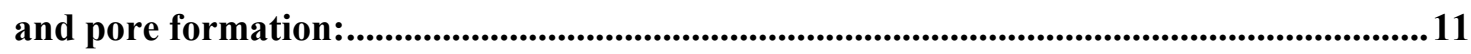

1.6 Cell selectivity of AMPs and role of cholesterol: ..........................................................13

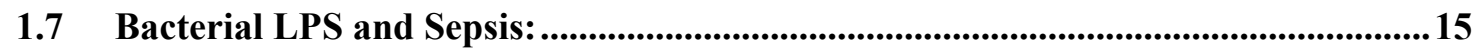

1.8 LPS as outer membrane permeability barrier: .......................................................17

1.9 Antiendotoxic antimicrobial peptides: ............................................................................. 18

1.9.1 Bactericidal permeability increasing protein (BPI): ............................................. 19

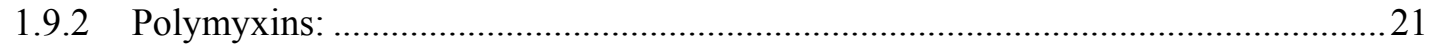

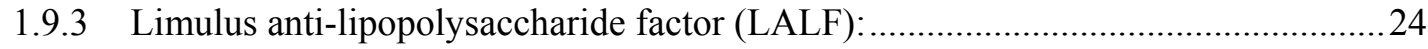

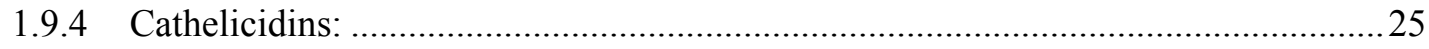

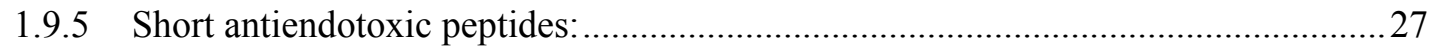

1.10 Strategies for the design of antimicrobial and antiendotoxic peptides: ..................28

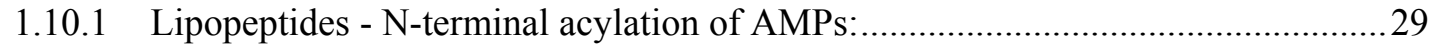

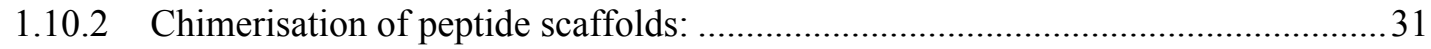

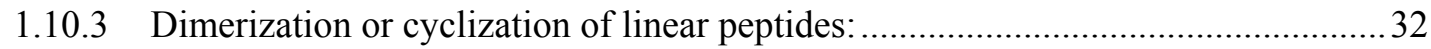

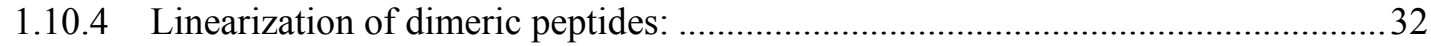

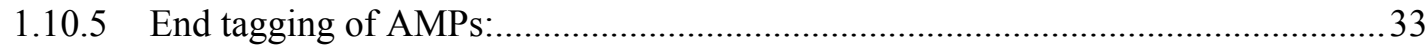




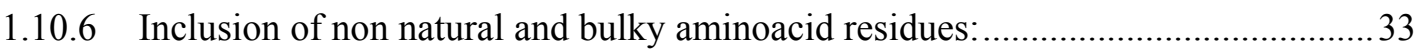

1.11 NMR structures aid in the design of novel antiendotoxic AMPs:...........................33

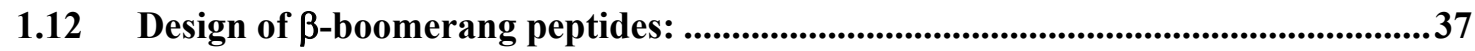

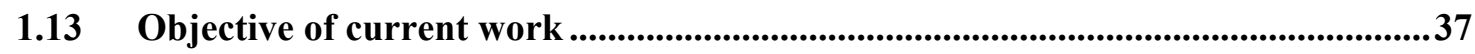

2 MATERIALS AND METHODS ……………....................................................42

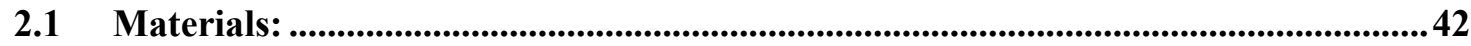

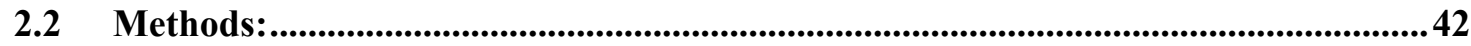

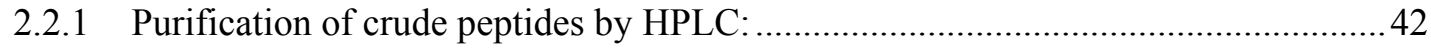

2.2.2 Oxidation of third generation $\beta$-boomerang hybrids: .......................................... 43

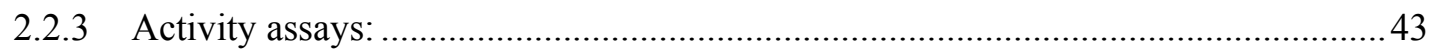

2.2.3.1 Minimum inhibitory concentration determination (MIC): .................................43

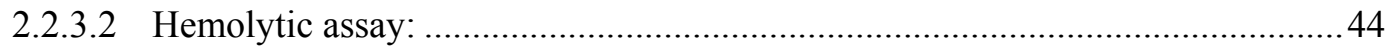

2.2.3.3 Limulus amoebocyte lysate (LAL) assay:........................................................ 44

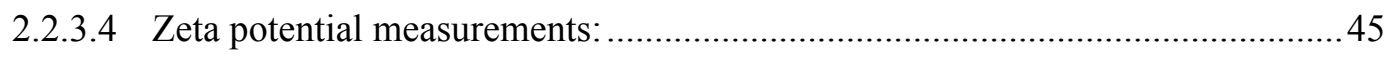

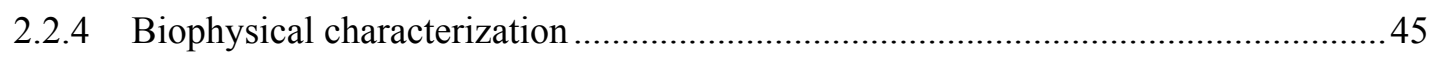

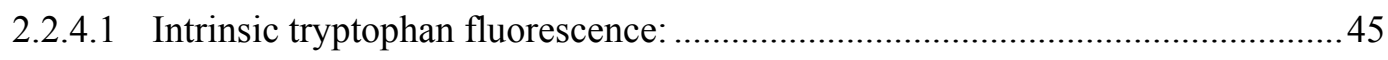

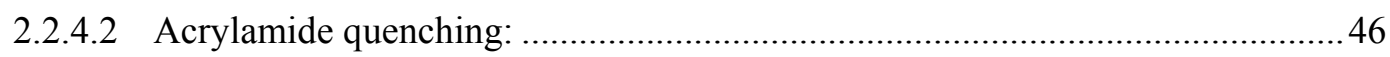

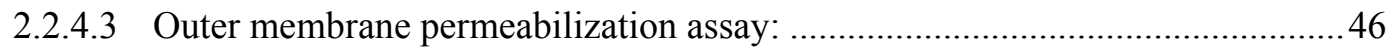

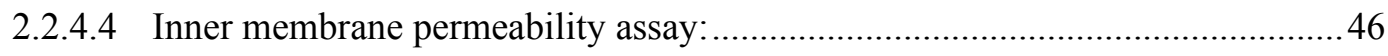

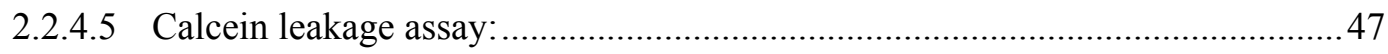

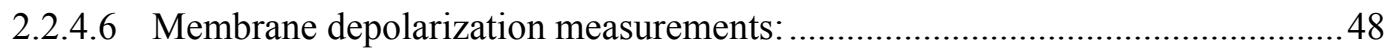

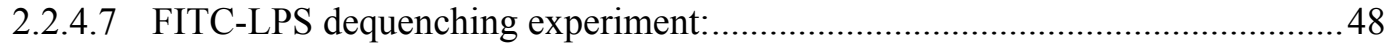

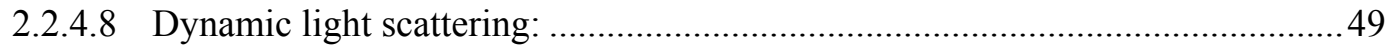

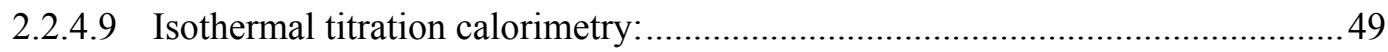

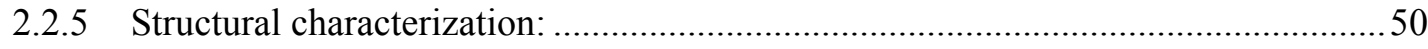

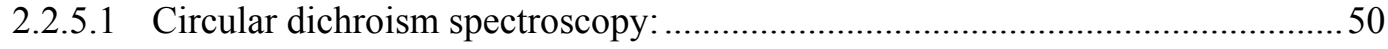

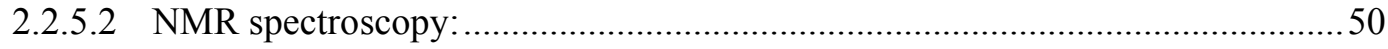

2.2.5.3 NMR derived structure calculations:.............................................................51

3 RESULTS AND DISCUSSION ................................................................................53

3.1 Dimerized $\beta$-boomerang lipopeptides ...........................................................................53

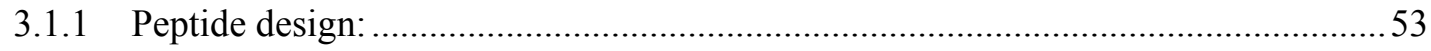

3.1.2 Dimerization of cystiene containing peptides and HPLC purification: .....................51

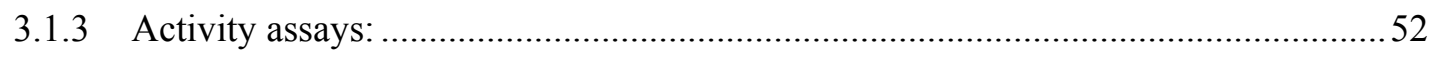


3.1.3.1 Determination of Minimum Inhibitory Concentration (MIC):...........................52

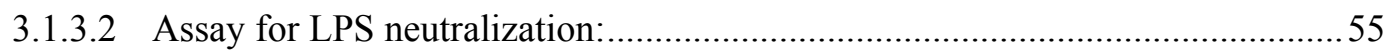

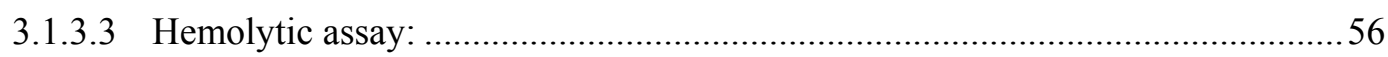

3.1.3.4 Surface charge measurements by Zeta potential: ..............................................57

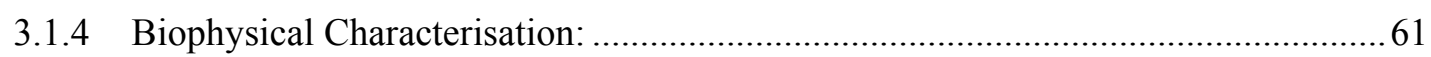

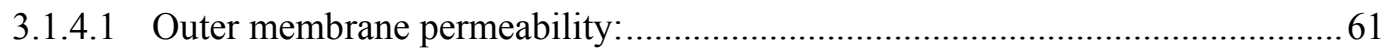

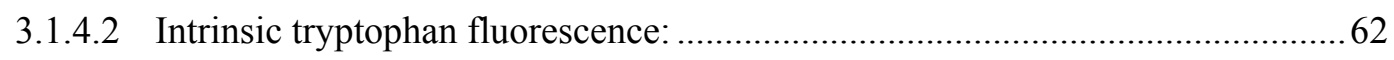

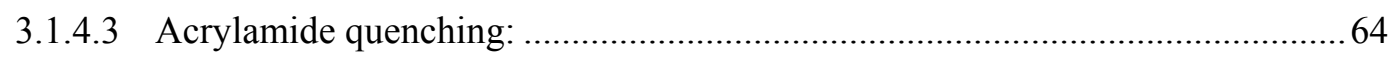

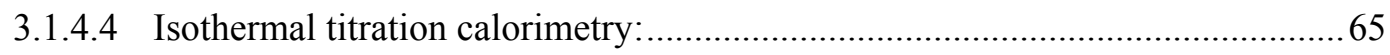

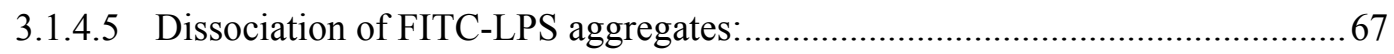

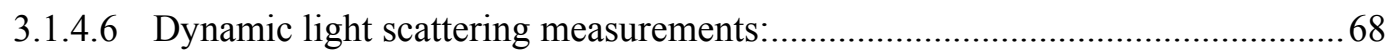

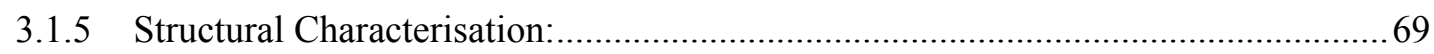

3.1.5.1 Solution NMR spectroscopy of representative cysteine and isoleucine

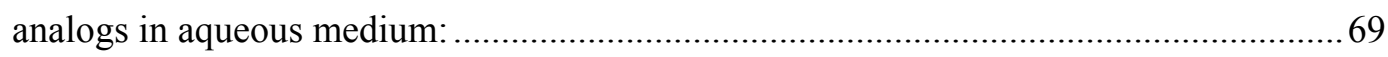

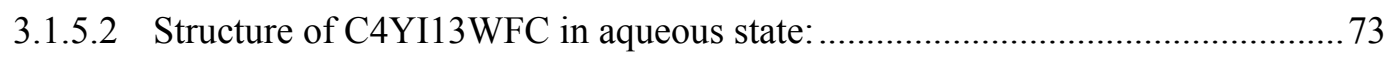

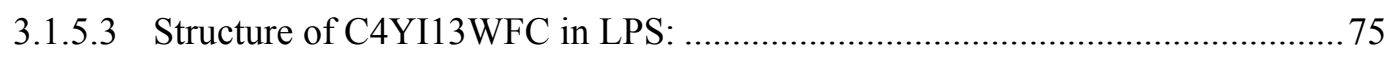

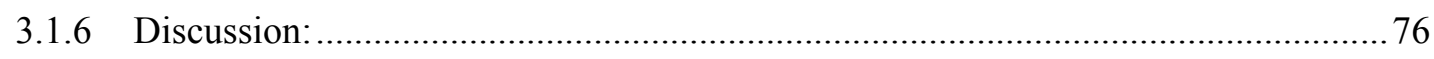

$3.2 \beta$-boomerang fold to conquer LPS trap...........................................................80

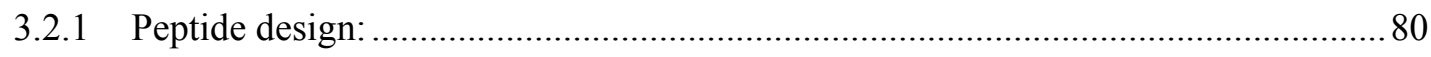

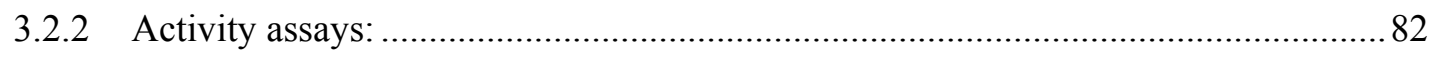

3.2.2.1 Minimum inhibitory concentration determination: ........................................ 82

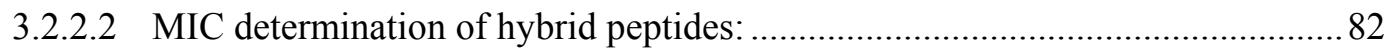

3.2.2.3 Determination of MIC for alanine and truncated mutants of LG21:................. 84

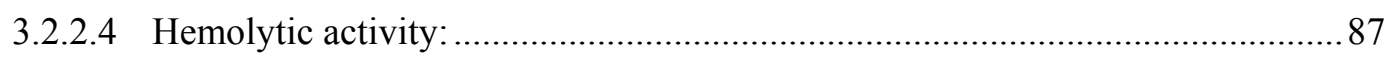

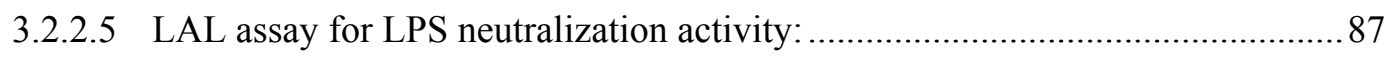

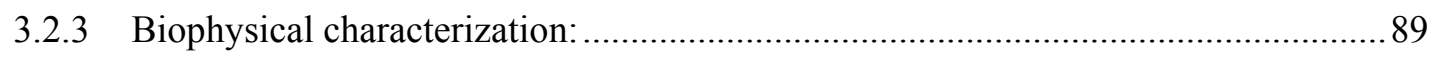

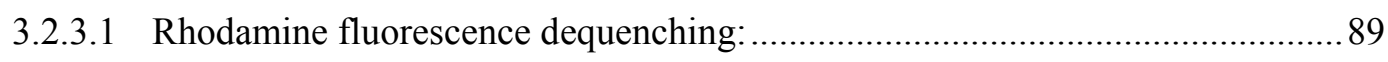

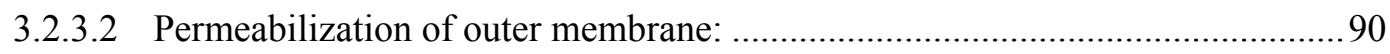

3.2.3.3 Permeabilization of both outer and inner membranes: ..................................... 91

3.2.3.4 Membrane permeabilization invitro - Calcein leakage assay: .......................... 92

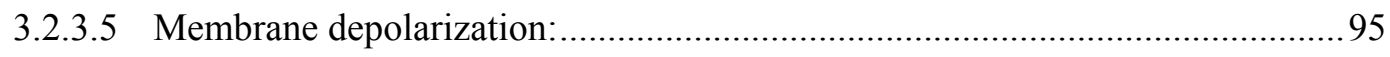

3.2.3.6 Intrinsic tryptophan fluorescence measurements: ....................................... 96

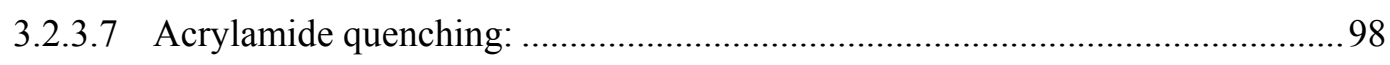

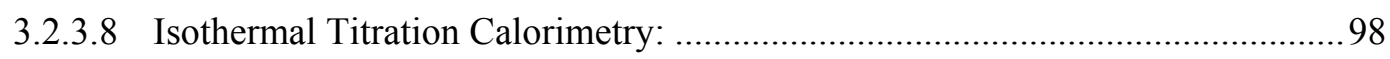




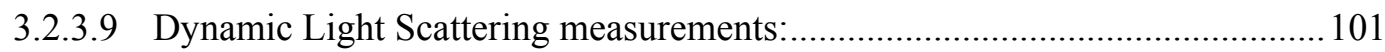

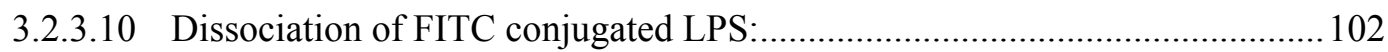

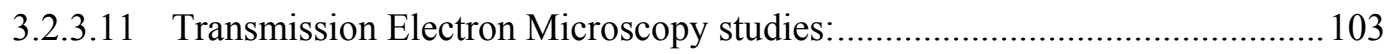

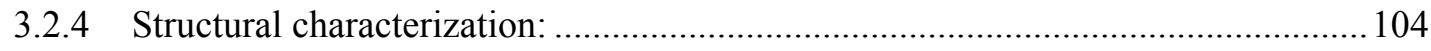

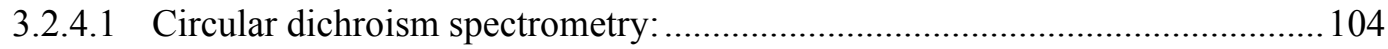

3.2.4.2 Nuclear Magnetic Resonance Spectroscopy: ................................................ 105

3.2.4.3 NMR studies of LG21 and LG21R19A in the presence of LPS micelles: ........ 106

3.2.4.4 Structural studies of LG21 in DPC micelles: .................................................. 108

3.2.4.5 Solution structure of LG21 in DPC micelles:................................................... 112

3.2.4.6 NMR spectroscopy of LG21R19A in DPC micelles:..................................... 116

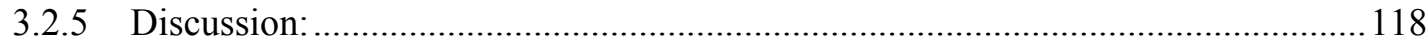

3.3 Cysteine deleted analogs of Protegrins ...............................................................121

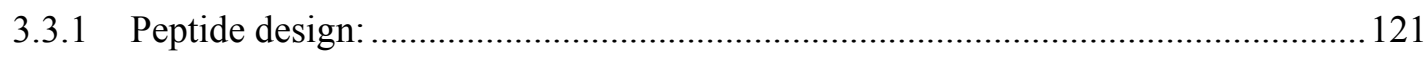

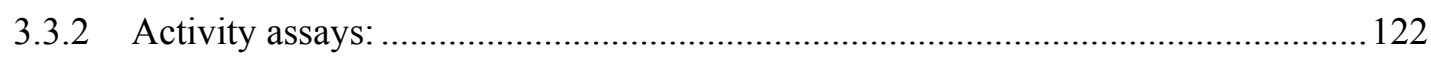

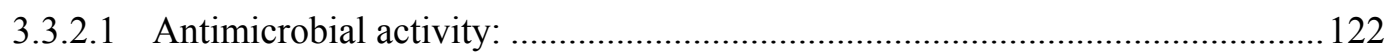

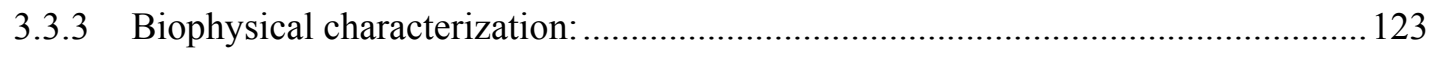

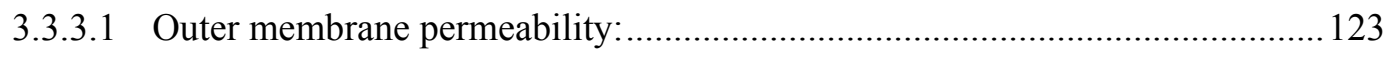

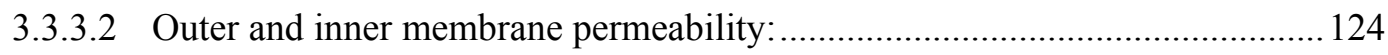

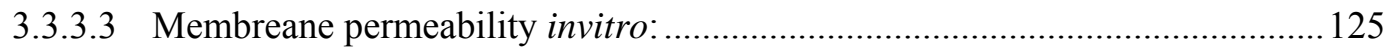

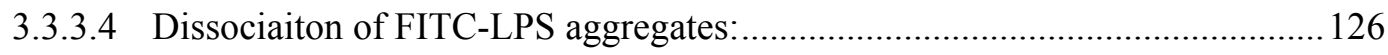

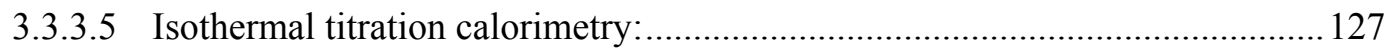

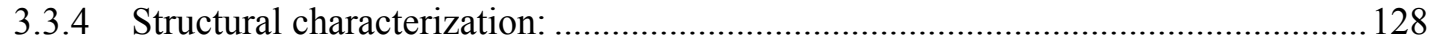

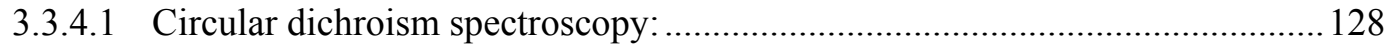

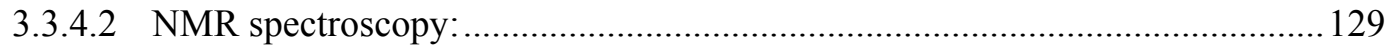

3.3.4.3 Three dimensional structures of the peptides in LPS micelles:........................ 135

3.3.4.4 Structure determination of peptides in DPC micelles: ...................................... 139

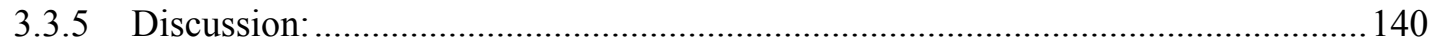

3.4 Design of arginine rich salt resistant peptides ............................................143

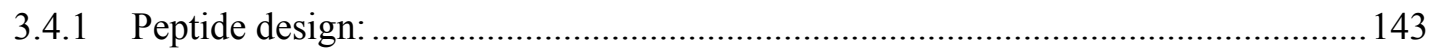

3.4.2 Minimum inhibitory concentration (MIC) determination:..................................... 145

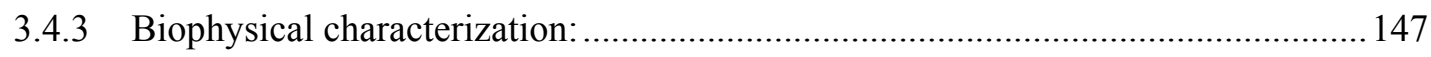

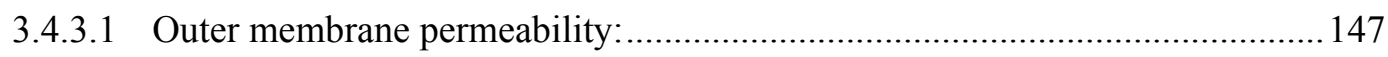

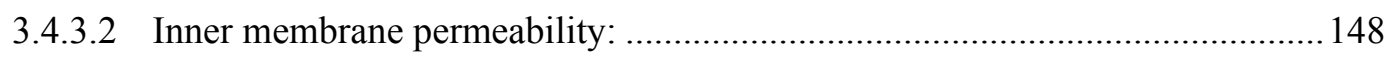

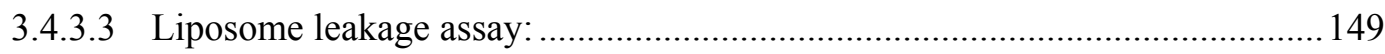


3.4.3.4 Dissociation of FITC-LPS aggregates:....................................................... 150

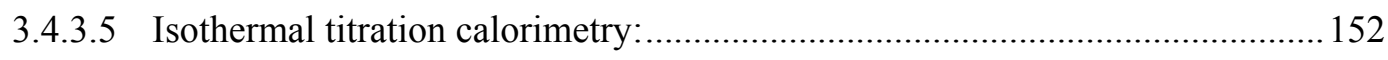

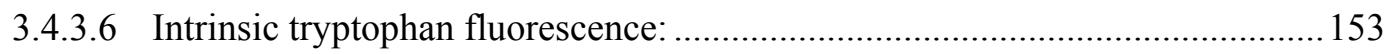

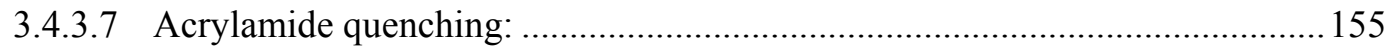

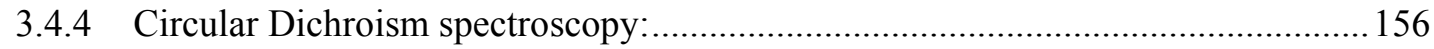

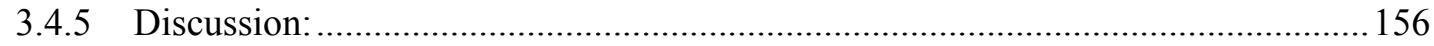

3.5 Conclusion summary …...........................................................................................159

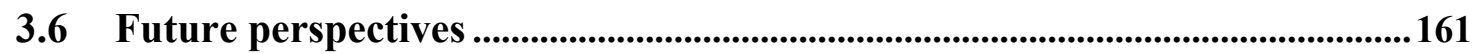

$\begin{array}{ll}\text { Appendix 1: } & 162\end{array}$

$\begin{array}{ll}\text { BIBLIOGRAPHY } & 163\end{array}$ 


\section{List of Figures}

\section{Chapter 1: Introduction}

Figure 1.1-1: Accelerated increase in the existence of drug resistant bacteria .................................2

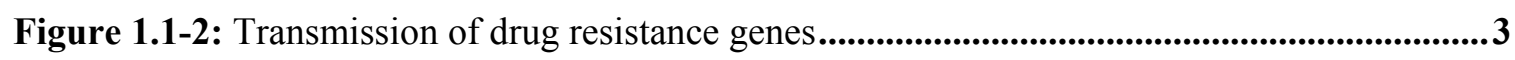

Figure 1.2-1: Structural diversity of AMPs. ..........................................................................................5

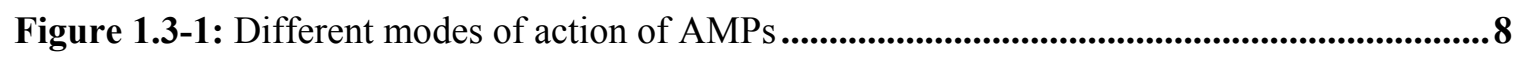

Figure 1.4-1: Interpretation of Gram negative .........................................................................................10

Figure 1.5-1: (A) Positive curvature strain or $\mathrm{H}_{\mathrm{I}}$ phase ....................................................................12

Figure 1.6-1: Mechanism of cell selectivity by AMPs ....................................................................14

Figure 1.7-1: Structure of Lipopolysaccharide .................................................................................15

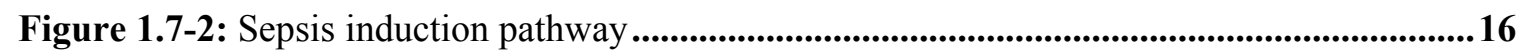

Figure 1.8-1: Heterogeneity of LPS ...................................................................................................18

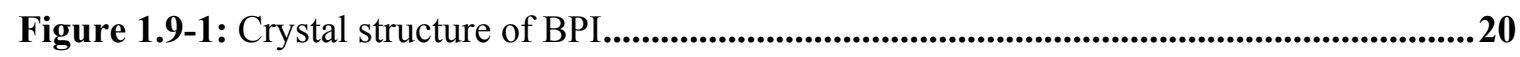

Figure 1.9-2: The N-terminal recombinant rBPI21 ................................................................................21

Figure 1.9-3: The basic molecular representation of Polymyxins ..............................................22

Figure 1.9-4: Three - dimensional structures of PMB in LPS ........................................................23

Figure 1.9-5: Crystal structure of LALF ........................................................................................24

Figure 1.9-6: Cathelicidins from different origin...........................................................................26

Figure 1.9-7: Three dimensional solution state NMR structures of short peptides.........................28

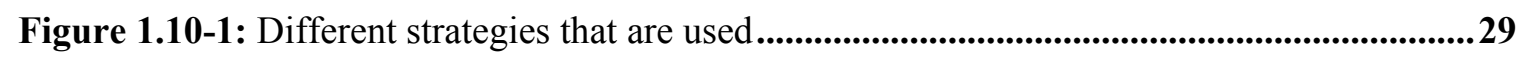

Figure 1.11-1: SAR studies of LF11, a human lactoferrin based peptide.........................................34

Figure 1.11-2: SAR studies of MSI594, a hybrid peptide of MSI78 and Meltitin..........................35 
Figure 1.12-1: Design of $\beta$-boomerang peptides

Figure 1.12-1: Design of $\beta$-boomerang peptides .....................................................................38

Figure 1.13-1: Objectives of current work...................................................................................................38

\section{Chapter 3: Results and Discussion}

\section{1: Dimerized $\beta$-boomerang lipopeptides}

Figure 3.1-1: A representative HPLC purification profile. .52

Figure 3.1-2: Bar diagrams representing the LPS neutralization activity .......................................56

Figure 3.1-3: Surface charge measurement of E.coli cells...........................................................60

Figure 3.1-4: Permeability of fluorescent probe, NPN ..........................................................61

Figure 3.1-5: Intrinsic tryptophan fluorescence measurement..............................................63

Figure 3.1-6: Binding profiles of isoleucine analogs and cysteine analogs...................................65

Figure 3.1-7: Dequenching of FITC fluorescence in FITC-LPS ..........................................67

Figure 3.1-8: Dynamic light scattering measurements ................................................................68

Figure 3.1-9: Sequence specific resonance assignment. ...................................................................70

Figure 3.1-10: Selected regions of two-dimensional ${ }^{1} \mathrm{H}-{ }^{1} \mathrm{H}$ NOESY spectra .................................71

Figure 3.1-11: Bar diagram summarizing NOEs .................................................................................72

Figure 3.1-12: Selected regions of two-dimensional ${ }^{1} \mathrm{H}-{ }^{1} \mathrm{H}$ NOESY spectra ..................................73

Figure 3.1-13: (A) Single model representation and (B) electrostatic surface representation ......75

Figure 3.1-14: Two dimensional ${ }^{1} \mathrm{H}-{ }^{1} \mathrm{H}$ spectra of C4YI13WFC free in the presence of LPS.....76

\section{2: $\beta$-boomerang fold to conquer LPS trap}

Figure 3.2-1: A bar diagram representing the LPS neutralization activity ..................................88

Figure 3.2-2: Rhodamine fluorescence dequenching...........................................................................90

Figure 3.2-3: Outer membrane permeabilizing ability......................................................................91 
Figure 3.2-4: Complete membrane disrupting ability.

Figure 3.2-5: Membrane permeabilization invitro ...................................................................................93

Figure 3.2-6: Membrane permeabilization invitro ..............................................................................94

Figure 3.2-7: Membrane depolarization of intact E.coli (A) and (B) spheroplasts........................95

Figure 3.2-8: Intrinsic tryptophan fluorescence measurement...........................................................97

Figure 3.2-9: Binding profiles of hybrid peptides........................................................................100

Figure 3.2-9: LPS micelle dissociation by hybrid peptides ...........................................................101

Figure 3.2-10: Dissociation of FITC conjugated LPS .....................................................................102

Figure 3.2-12: Electron micrographs of E.coli ................................................................................103

Figure 3.2-13: Far-UV CD spectra ...........................................................................................................105

Figure 3.2-14: Low field amide region of 1-D proton spectra of LG21 .......................................106

Figure 3.2-15: Overlay of two-dimensional ${ }^{1} \mathrm{H}-{ }^{1} \mathrm{H}$ NOESY spectra of $0.5 \mathrm{mM}$ LG21 ............... 107

Figure 3.2-16: Comparison of the shift in indole ring proton of tryptophan ................................108

Figure 3.2-17: Sequence specific resonance assignment DPC bound LG21...............................109

Figure 3.2-18: NH-NH region of two dimensional ${ }^{1} \mathrm{H}-{ }^{1} \mathrm{H}$ NOESY spectrum .............................109

Figure 3.2-19: (A) Resonance assignment (B) spectra showing aliphatic-aromatic side chain..110

Figure 3.2-20: Right side: R19 residue experiencing abnormal ...............................................111

Figure 3.2-21: Difference of observed chemical shift deviations ..................................................112

Figure 3.2-22: Bar diagram representing NOE summary .................................................................114

Figure 3.2-23: Superimposition of backbone of 20 lowest energy structures...............................115

Figure 3.2-24: Surface presentation of LG21 in DPC micelles ...................................................116

Figure 3.2-25: Resonance assignment of two dimensional.........................................................117 
Figure 3.2-26: Model explaining the rationale behind the synthesis of hybrid peptides.

\section{3 : Cysteine deleted analogs of Protegrins}

Figure 3.3-1: Outer membrane permeability of deleted analogs of PG-1 in E.coli

Figure 3.3-2: Complete membrane disrtuption by deleted analogs of PG-1 in E.coli cells........125

Figure 3.3-3: Membrane permeabilization invitro by deleted analogs of PG-1..........................126

Figure 3.3-4: Dissociation of FITC conjugated ...................................................................................126

Figure 3.3-5: Thermondynamic binding profiles of cysteine deleted analogs of PG-1 ..............127

Figure 3.3-6: Far UV CD spectra.................................................................................................................129

Figure 3.3-7: Sequence specific resonance assignment.................................................................130

Figure 3.3-8: Selected region of two dimensional ........................................................................131

Figure 3.3-9: NOE summary of RR14 in LPS micelles..............................................................132

Figure 3.3-10: (A) Selected region of two dimensional ${ }^{1} \mathrm{H}-{ }^{1} \mathrm{H}$ tr-NOESY spectrum ...................133

Figure 3.3-11: (A) NOE summary of RR11 in LPS micelles. ..................................................134

Figure 3.3-12: (A) Sequnece specific assignment of aminoacid residues of LR10 .....................134

Figure 3.3-13: (A) NOE summary of LR10 in LPS micelles. ............................................................135

Figure 3.3-14: Ensemble of twenty lowest energy structures .................................................137

Figure 3.3-15: Single model representations ............................................................................................138

Figure 3.3-16: Electrostatic surface representation.......................................................................139

Figure 3.3-17: Selected region of two dimensional ${ }^{1} \mathrm{H}-{ }^{1} \mathrm{H}$ NOESY spectra ..................................140

\section{4: Design of arginine rich salt resistant peptides}

Figure 3.4-1: Helical wheel projection of designed peptides............................................................144 
Figure 3.4-2: Outer membrane permeability of mid log phase grown E.coli cells

Figure 3.4-3: Inner membrane permeability of mid log phase grown E.coli cells......................149

Figure 3.4-4: Leakage of calcein from POPC:LPS LUVs .................................................................150

Figure 3.4-5: Dissociation of FITC-LPS aggregates ................................................................151

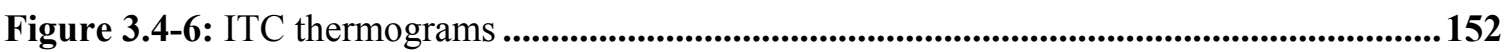

Figure 3.4-6: Intrinsic tryptophan fluorescence measurement of designed peptides ...................154

Figure 3.4-8: Far UV CD measurements ............................................................................156 


\section{List of Tables}

Table 1.4-1: Composition of different lipids.........................................................................11

Table 2.2-1: Bacterial strains and respective ATCC numbers.....................................................43

Table 3.1-1: Third generation of $\beta$-boomerang peptides and their primary structure....................54

Table 3.1-2: Retention time of the peptides purified using HPLC .............................................53

Table 3.1-3: Minimum inhibitory concentration (in $\mu \mathrm{M}$ ) .......................................................54

Table 3.1-4: Minimum inhibitory concentration (in $\mu \mathrm{M})$..............................................................54

Table 3.1-5: Percentage of hemolysis ........................................................................................57

Table 3.1-6: Difference in the emission maximum of $\operatorname{Trp}$ fluorescence......................................63

Table 3.1-7: Quenching of tryptophan residues of the peptides by acrylamide..............................64

Table 3.1-8: Thermodynamic parameters of cystiene and isoleucine analogs...............................66

Table 3.1-9: Dissociation of aggregates of LPS micelles .........................................................69

Table 3.1-10: Summary of structural statistics of 20 lowest energy structures..............................74

Table 3.2-1: Primary structure of designed peptides.................................................................................81

Table 3.2-2: Minimum inhibitory concentration(in $\mu \mathrm{M})$ determination ...........................................83

Table 3.2-3: Comparison of MIC (in $\mu \mathrm{M})$ values for hybrids with Temporins ............................83

Table 3.2-4: Minimum inhibitory concentration (in $\mu \mathrm{M})$...............................................................84

Table 3.2-5: MIC values (in $\mu \mathrm{M})$ of truncated LG21 mutants .......................................................85

Table 3.2-6: MIC values (in $\mathrm{mM}$ ) of alanine mutants of LG21 .......................................................85

Table 3.2-7: MIC values (in $\mathrm{mM}$ ) of alanine mutants of LG21 .............................................86

Table 3.2-8: Percentage of hemolysis of peptides......................................................................................87

Table 3.2-9: Difference in the emission maximum of $\operatorname{Trp}$ fluorescence $(\Delta \lambda \max )$ 
Table 3.2-10: Stern-Volmer (Ksv) quenching constants..........................................................98

Table 3.2-11: Thermodynamic parameters for hybrid peptides ......................................................99

Table 3.2-11: Dissociation of variously sized LPS micelles...........................................................102

Table 3.2-13: Table summarizing structural statistics of 20 lowest energy structures ................113

Table 3.3-1: Peptides designed based on Protegrin PG-1 and their aminoacid sequences. .........121

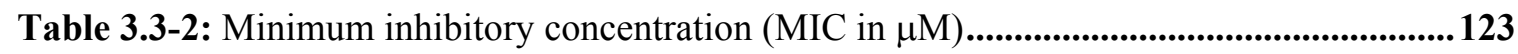

Table 3.3-3: Calculation of thermodynamic parameters ............................................................128

Table 3.3-4: Summary of structural statistics of 20 lowest energy structures...............................136

Table 3.4-1: Primary structure of designed peptides used in this study.......................................143

Table 3.4-2: Determination of MIC $(\mu \mathrm{M})$ values for designed peptides.......................................146

Table 3.4-3: Determination of MIC $(\mu \mathrm{M})$ values for designed peptides.......................................146

Table 3.4-4: Calculation of thermodynamic parameters ...................................................................153

Table 3.4-4: Emission maximum of Trp fluorescence $(\lambda \max )$ of peptides....................................154

Table 3.4-5: Quenching of tryptophan residue......................................................................155 


\section{Publications.}

1. Mohanram H, Bhattacharjya S. Salt resistant alpha-helical antimicrobial peptides. (Manuscript under preparation).

2. Mohanram H, Bhattacharjya S. Cysteine Deleted Protegrin-1 (CDP-1): Antibacterial Activity, Outer-Membrane Disruption and Selectivity. (Manuscript under revision).

3. Mohanram H, Bhattacharjya S. $\beta$-Boomerang Antimicrobial and Antiendotoxic Peptides: Lipidation and Disulfide Bond Effects on Activity and Structure. Pharmaceuticals (Basel). 2014 Apr 21;7(4):482-501.

4. Mohanram H, Bhattacharjya S. Resurrecting inactive antimicrobial peptides from the lipopolysaccharide trap. Antimicrob Agents Chemother. 2014 Apr;58(4):1987-96.

5. Saravanan R, Joshi M, Mohanram H, Bhunia A, Mangoni ML, Bhattacharjya S. NMR structure of temporin-1 ta in lipopolysaccharide micelles: mechanistic insight into inactivation by outer membrane. PLoS One. 2013 Sep 9;8(9):e72718.

6. Saravanan R, Li X, Lim K, Mohanram H, Peng L, Mishra B, Basu A, Lee JM, Bhattacharjya S, Leong SS. Design of short membrane selective antimicrobial peptides containing tryptophan and arginine residues for improved activity, salt-resistance, and biocompatibility. Biotechnol Bioeng. 2014 Jan;111(1):37-49.

7. Mohanram H, Nip A, Domadia PN, Bhunia A, Bhattacharjya S. NMR structure, localization, and vesicle fusion of Chikungunya virus fusion peptide. Biochemistry. 2012 Oct 9;51(40):7863-72.

8. Liu F, Soh Yan Ni A, Lim Y, Mohanram H, Bhattacharjya S, Xing B. Lipopolysaccharide neutralizing peptide-porphyrin conjugates for effective photoinactivation and intracellular imaging of gram-negative bacteria strains. Bioconjug Chem. 2012 Aug 15;23(8):1639-47.

9. Saravanan R, Mohanram H, Joshi M, Domadia PN, Torres J, Ruedl C, Bhattacharjya S. Structure, activity and interactions of the cysteine deleted analog of tachyplesin-1 with lipopolysaccharide micelle: Mechanistic insights 
into outer-membrane permeabilization and endotoxin neutralization. Biochim Biophys Acta. 2012 Jul;1818(7):1613-24.

10. Bhunia A, Mohanram H, Bhattacharjya S. Structural determinants of the specificity of a membrane binding domain of the scaffold protein Ste5 of budding yeast: implications in signaling by the scaffold protein in MAPK pathway. Biochim Biophys Acta. 2012 May;1818(5):1250-60.

11. Bhunia A, Saravanan R, Mohanram H, Mangoni ML, Bhattacharjya S. NMR structures and interactions of temporin-1Tl and temporin-1Tb with lipopolysaccharide micelles: mechanistic insights into outer membrane permeabilization and synergistic activity. J Biol Chem. 2011 Jul 8;286(27):24394-406.

12. Domadia PN, Li YF, Bhunia A, Mohanram H, Tan SM, Bhattacharjya S. Functional and structural characterization of the talin F0F1 domain. Biochem Biophys Res Commun. 2010 Jan 1;391(1):159-65.

13. Bhunia A, Mohanram H, Domadia PN, Torres J, Bhattacharjya S. Designed beta-boomerang antiendotoxic and antimicrobial peptides: structures and activities in lipopolysaccharide. J Biol Chem. 2009 Aug 14;284(33):219912004.

14. Bhunia A, Tang XY, Mohanram H, Tan SM, Bhattacharjya S. NMR solution conformations and interactions of integrin alphaLbeta2 cytoplasmic tails. J Biol Chem. 2009 Feb 6;284(6):3873-84.

15. Bhunia A, Mohanram H, Bhattacharjya S. Lipopolysaccharide bound structures of the active fragments of fowlicidin-1, a cathelicidin family of antimicrobial and antiendotoxic peptide from chicken, determined by transferred nuclear Overhauser effect spectroscopy. Biopolymers. 2009;92(1):9-22.

16. Bhunia A, Domadia PN, Mohanram H, Bhattacharjya S. NMR structural studies of the Ste11 SAM domain in the dodecyl phosphocholine micelle. Proteins. 2009 Feb 1;74(2):328-43. 


\section{ABBREVIATIONS}

ATCC

AMP

MDR

FDA

LB

$\mathrm{MH}$

MIC

$\mathrm{RBC}$

LPS

$\mathrm{KDO}$

EU

EDTA

DPC

SDS

FITC-LPS

Rho

NPN

Di-S-C3

LAL

GUV

LUV

SUV

POPC

POPG

HPLC

TFA

ITC

CD

DLS

TEM
- American Type Culture Collection

- Anti-microbial Peptides

- Multi Drug Resistance

- Food and Development Authority

- Luria-Bertanii broth

- Mueller-Hinton broth

- Minimum Inhibitory Concentration

- Red Blood Cells

- LipoPolySaccharides

- Keto Deoxy Octulosonate

- Endotoxin Units

- EthyleneDiamineTetraAcetic acid

- DodecylPhosphoCholine

- Sodium Dodecyl Sulfate

- Fluorescein IsoThioCyanate LPS

- Rhodamine

- 1-N-Phenyl Naphtylamine

- 3,3`-DiethylthiodicarboCyanine iodide

- Limulus Ameoboctye Lysate

- Giant Unilamellar Vesicles

- Large Unilamellar Vesicles

- Small Unilamellar Vesicles

- 1-palmitoyl-2-oleoyl-sn-glycero-3-phosphocholine

- 1-palmitoyl-2-oleolyl-sn-glycero-3-phosphoglycerol

- High Performance Liquid Chromatography

- TriFluoroAceticacid

- Isothermal Titration Calorimetry

- Circular Dichroism

- Dynamic Light Scattering

- Transmission Electron Microscopy 


$\begin{array}{ll}\text { NMR } & \text { - Nuclear Magnetic Resonance spectroscopy } \\ \text { STD-NMR } & \text { - Saturation Transfer Difference NMR } \\ \text { NOESY } & \text { - Nuclear Overhauser SpectroscopY } \\ \text { TOCSY } & \text { - TOtal Correlation SpectroscopY } \\ \text { Tr-NOESY } & \text { - Transfer NOESY } \\ \text { 1-D } & \text { - One-Dimensional } \\ 2-D & \text { - Two-Dimensional } \\ { }^{1} \mathrm{H} & \text { - Proton } \\ \text { NOE } & \text { - Nuclear Overhauser Effect } \\ \text { DSS } & \text { - 2,2-Dimethyl-2-Silapentane 5-Sulfonate sodium salt } \\ \text { E.coli } & \text { - Escherichia coli } \\ \text { P.aeruginosa } & \text { - Pseudomonas aeruginosa } \\ \text { Trp } & \text { - Tryptophan } \\ \text { Arg } & \text { - Arginine } \\ \mathrm{K}_{\mathrm{a}} & \text { - Association constant } \\ \mathrm{K}_{\mathrm{d}} & \text { - Dissociation constant } \\ \mathrm{K}_{\mathrm{sv}} & \text { - Stern-Volmer constant } \\ \mathrm{mM} & \text { - milliMolar } \\ \mu \mathrm{M} & \text { - microMolar } \\ \text { HBD } & \text { - Human Beta Defensins } \\ \text { BPI } & \text { - Bactericidal Permeability Increasing protein } \\ \text { LBP } & \text { - Lipopolysaccharide Binding Protein } \\ \text { CD14 } & \text { - Cluster of Differentiation 14 } \\ \text { TLR } & \text { - Toll Like Receptor } \\ \text { PBS } & \text { - Phosphate Buffered Saline } \\ & \end{array}$




\section{ABSTRACT}

The discovery of antibiotics and its incredible usage in saving human lives has been considered as one of the major discoveries in medicine. However, fight against bacteria has not ended due to the emergence of resistant microbes. Super bugs or Multi- drug resistance (MDR) pathogens have become a common occurrence now. The situation is further complicated by the outburst of new diseases like H1N1, Dengue etc and its associated illnesses. The immune suppression of host system during such illness also invites other hospital infections and hence the treatment involves usage of multiple drugs. The mode of action of antibiotics is receptor mediated and hence there are high chances for the microbes to mutate such receptors and become resistance to that drug. Under such circumstances, the discovery of antimicrobial peptides incited a new path in development of future drugs, which the microbes would find it difficult to develop resistance.

Lipopolysaccharides (LPS) are present in the outer layer of outer membrane of Gram negative bacteria. It serves dual role, as an important component in maintaining structural integrity of bacteria and also as membrane impermeability barrier to antibiotics and other hydrophobic substances. LPS is released in to the blood stream either during cell division or by the action of antibiotics. Such circulating LPS when accumulated in higher amounts were recognized to stimulate inflammatory pathway provoking septic shock syndrome. Sepsis is identified as one of the major mortality causing condition in hospital Intensive care units (ICU). Activity studies of antimicrobial peptides (AMPs) revealed that they could bind and neutralize the LPS before exerting its killing activity. Hence these AMPs from natural sources can be used as template for the synthesis of effective antiendotoxic antimicrobial peptides.

In the current work, antimicrobial peptides were designed to characterize the structural features that govern the endotoxin neutralization and bacterial killing. First and second generation $\beta$-boomerang peptides showed promising antimicrobial activity with LPS neutralization. Since N-terminal acylation and dimerization was found to increase antimicrobial activity. $\beta$-boomerang peptides were acylated and dimerized through disulfide bridges in third generation. Isoleucine analogs (devoid of cysteine mediated disulphide link, YI13WF) and Cysteine analogs (YI13WFC) were further 
characterized for antiendotoxic antimicrobial activity. Both the analogs were also acylated with different length of acyl chains to define the appropriate hydrophobicity limit for the activity of the lipopeptides thus designed. Structure- activity correlation studies of the peptides explained that the cysteine analogs exhibited more efficient anti microbial activity than the isoleucine analogs. Further, the dimerized lipopeptides (acylated cysteine analogs) demonstrated broad spectrum of antibacterial activity with efficient LPS perturbation. Among the different chain lengths for acylation, C4 was found to be more appropriate as the increase in hydrophobicity complemented with antimicrobial antiendotoxic activity with reduced hemolytic activity. Atomic resolution structure of C4YI13WFC in aqueous state revealed that the peptide folds into characteristic boomerang shape with disulfide linkage between two cysteine residue bringing the residues $\mathrm{W} 4$ and F12 close together to form the aromatic fold.

In the second project, the structured LPS binding motif (GG8WF) identified in second generation of $\beta$-boomerang peptides was conjugated with the peptides that are found to aggregate on LPS layer, but active once trespasses the LPS layer. GG8WF peptide also found to fold into characteristic boomerang shape with aromatic fold between W2 and F7 upon interaction with LPS. GG8WF peptide was conjugated at C-terminus of Temporin A, Temporin B and synthetic K5L7 peptides that are found to aggregate on LPS layer. Three dimensional structure determination of the hybrid peptide revealed that the peptide folds into alpha helix upon membrane interaction and the positive charges in GG8 peptide initiates the binding of peptide with outer membrane. It was also found that the conjugation rendered alpha helix to boomerang motif also, but instead of the conserved aromatic lock between W2 and F7, cation- $\pi$ interaction between W15 and R19 (in hybrid peptides) accounted for the remarkable activity of the hybrid peptides. When this R19 was mutated to alanine, it produced an inactive peptide.

In the third project, cysteine deleted analogs of Protegrin-1 peptide, isolated from porcine leukocytes were characterized. The full length analog without cysteine, RR14 was found to be active followed by truncated analogs RR11 and LR10. The beta hairpin fold with $\pi$ - $\pi$ interactions between Tyr and Phe was found to be conserved, even after deletion of cysteine residue. SAR studies of these analogs revealed that the 
beta hairpin fold is important in exerting the antibacterial activity and that the first four residues RGGR provides the initial ionic interaction with outer membrane. When RGGR was deleted, the peptide showed impaired antibacterial activity.

In the last project, antimicrobial peptides rich in arginine with appropriately placed tryptophan, leucine and isoleucine residues were designed. Increase in hydrophobic surface of the peptide confers salt resistance to antimicrobial peptides. Pharmacological development of antimicrobial peptides requires the peptide to be salt tolerant. These series of designed peptides were found to be salt tolerant and adopt alpha helix upon interaction with LPS outer membrane. 


\section{CHAPTER 1}

\section{INTRODUCTION}




\section{INTRODUCTION}

\subsection{Antibiotic resistance and emergence of Antimicrobial peptides (AMPs):}

Penicillin was one of the first antibiotics to be discovered by Alexander Fleming. Even though Penicillin was discovered as early as 1928, the successful application of penicillin as therapeutic agent was developed only in 1940 [1]. But preceding its introduction, penicillinase synthesizing bacteria were reported in 1940s [2]. In the same manner, resistant strains of bacteria to sulfonamides and streptomycin were reported soon after its introduction as successful therapeutic agents [1]. From then the number of multiple drug resistant (MDR) bacteria were found to increase with decrease in the number of antibiotics being approved by FDA (Figure 1.1-1).
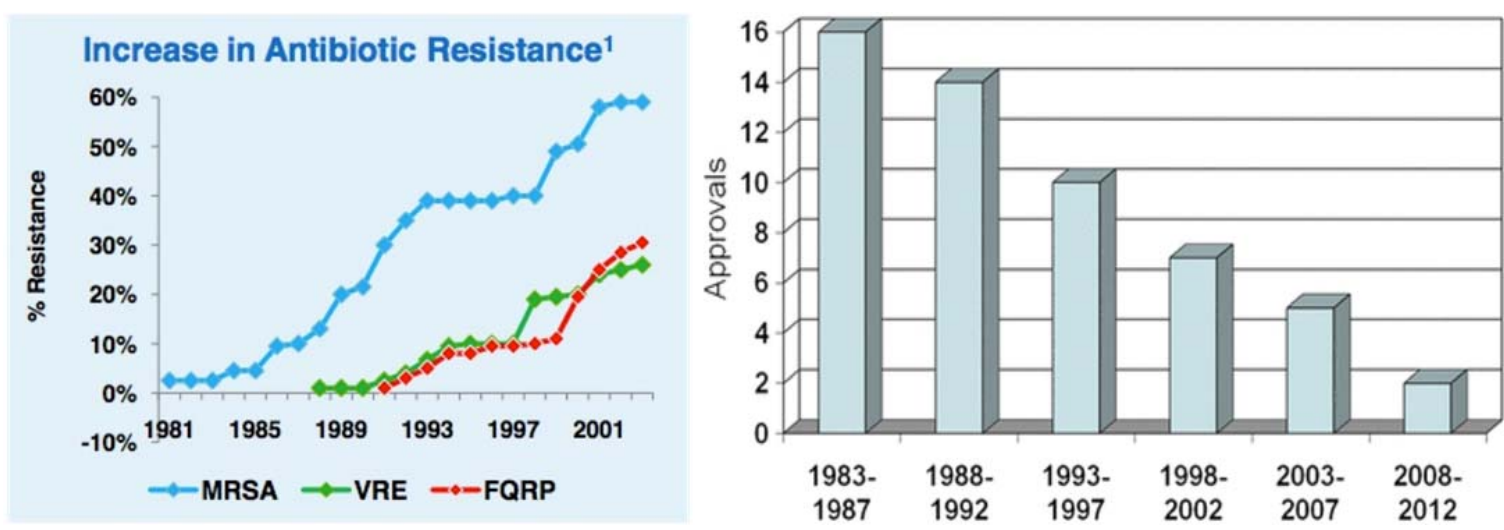

Figure 1.1-1: Accelerated increase in the existence of drug resistant bacteria and creeping decrease in the approval of drugs by FDA (U.S.A). Taken from ref [3], [4].

It was thought that the antibiotic resistance crops up from over or unwanted usage of drugs. The antibiotics are not only used for treating diseases in humans but also in food providing animals for control and combat diseases they develop. Hence the more the usage of antibiotics, the chances are more likely to develop resistance [5]. The existence of penicillinase even before its introduction as therapeutic agent surprised the researchers that the resistance ' $r$ ' genes exist naturally in communities of bacteria [1]. 
One of the prominent mechanisms of procurement of antibiotic resistance is horizontal gene transfer (Figure 1.1-2) [6]. When bacteria carrying ' $r$ ' genes co-exist with bacteria that are susceptible to certain antibiotics, gene transfer occurs via conjugation or transformation. The transfer of genes can also occur through the phages that have acquired ' $r$ ' genes while infecting resistant bacteria. These phages then transmit the resistance genes to susceptible bacteria through transduction. Spontaneous mutation can also help to generate resistance genes [6]. Apart from this extrinsic acquisition of resistant genes, antibiotic resistance may also stem from drug efflux mechanisms, altered drug receptors or synthesis of metabolic pathways that develop resistance [7].

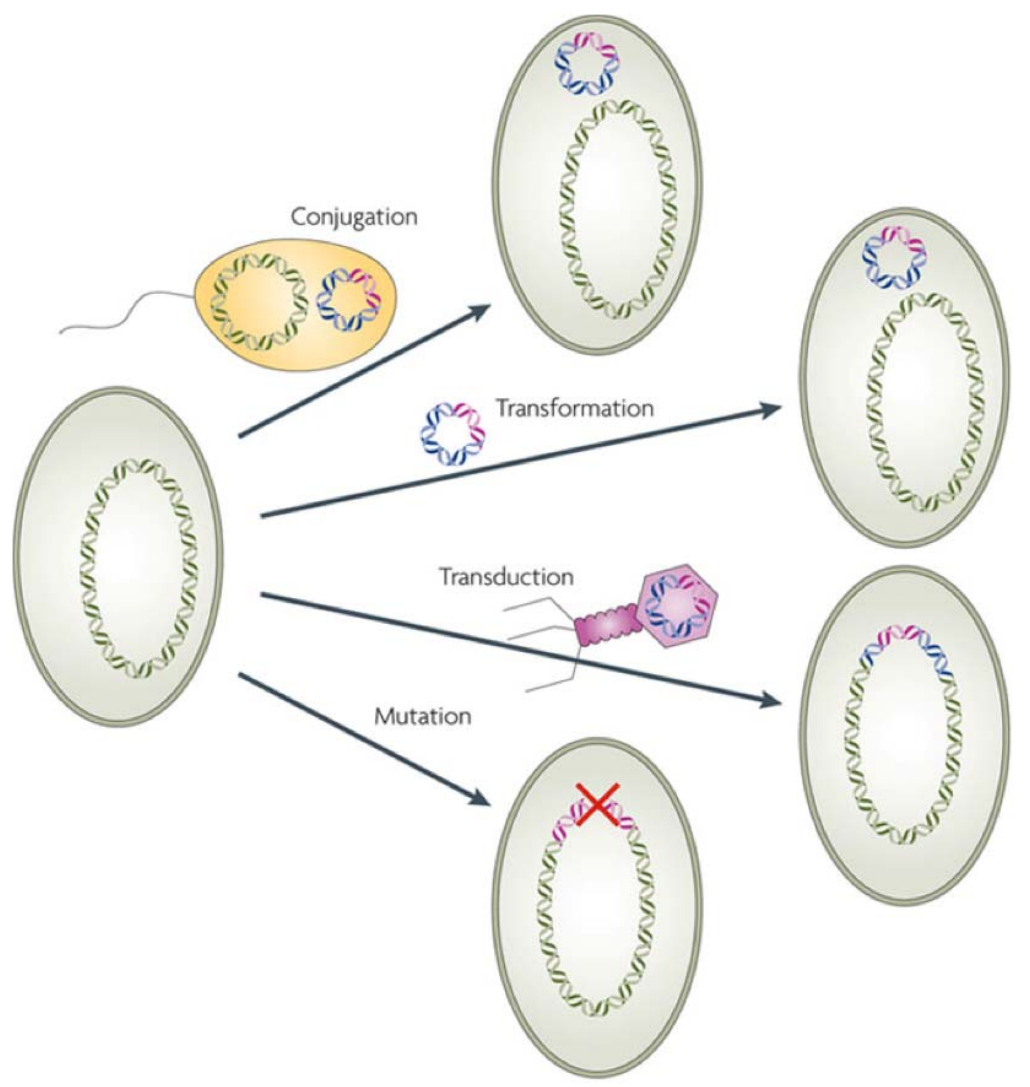

Figure 1.1-2: Transmission of drug resistance genes: Antibiotic resistance can spread through the horizontal gene transfer (HGT) via conjugation or transformation of resistance gene carrying bacteria with other susceptible bacteria, transduction through phages or by random mutation of hotspots. Taken from ref [6]. 
Cationic antimicrobial peptides (AMPs) are the compounds that are secreted by a wide variety of living organisms as endogenous antibiotics [8]. The milestone inventions of Melittin [9], Cecropins [10], Defensins [11], Magainins [12] blossomed as new hope of life when the world has been grieving with antibiotic resistance. In the competition of the survival of the fittest, human kind is exposed to wide variety of microbes in day-to-day life. Yet the infection is established only when the infectious agents reach a threshold concentration. The primary line of defense against such infectious agents is brought about by innate immune mechanism and these antimicrobial peptides are identified as one of such compounds involved in host defense [13]. AMPs are preferred over antibiotics because of its specific mode of action. AMPs act via non receptor mediated mechanism where the target is either the membrane or any intracellular component of microbes [14].

\subsection{Structural diversity of AMPs:}

Antimicrobial peptides belong to diverse group of peptides characterized by broad spectrum of activity. They are typified by short sequences with appropriate proportion of polar and hydrophobic aminoacid residues [14]. Hence at physiological $\mathrm{pH}$, the

presence of Arg and Lys residues imparts a net positive charge that helps the peptide to get attracted towards negatively charged bacterial membranes [14]. Amphipathicity i.e. segregation of hydrophilic and hydrophobic residues into different faces of the peptide conformation, is also considered as one of the important characters of AMPs [15].

The AMPs are structurally divergent that they can be categorized based on the structures they adopt upon membrane interaction [16]. The four most prominent ones being (a) $\alpha$-helix, (b) $\beta$-sheets stabilized by disulphide bridges (c) extended and (d) loops with or without disulphide bonds (Figure 1.2-1).

\subsubsection{Alpha helical antimicrobial peptides:}

Most naturally available AMPs adopt alpha helical conformation upon interaction with membranes [17]. They are mostly short, linear and folds in to amphipathic structures required for innate defense [18]. These peptides mostly exist in random coil 
conformation in aqueous environment because of the competition that exist between hydrogen bond of the solvent with its own hydrogen bonds [19]. In other words, the peptides cannot afford the entropy loss when it folds in to helix in aqueous environment. Upon interaction with membranes, the peptide enters into hydrophobic environment in stabilized conformation [19].

\begin{tabular}{c}
\multicolumn{3}{c}{ Structural diversity of antimicrobial peptides } \\
\hline Helix
\end{tabular}

Figure 1.2-1: Structural diversity of AMPs: Representative model of helix as in Magainin-2 (PDB ID: 2MAG); helical hairpin as in MSI-594 (PDB ID: 2K98); $\beta$ sheet with 2 disulphide bridges as in Protegrin-1 (PDB ID: 1PG1); $\beta$-sheet with 3 disulphide bridges as in human $\beta$-defensin-2 (PDB ID: 1FQQ); extended structure as in Indolicidin (PDB ID: 1G89); disulphide loop as in Thanatin (PDB ID: 8TFV) and loop structure as in Gramicidin A (PDB ID: 1MAG).

Magainins are a group of AMPs, isolated from frog skin in 1987, exhibit broad spectrum of activity against microbes [12]. Solid state NMR studies of magainin 2 revealed that the peptide adopted complete alpha helix that lies parallel to membrane 
(Figure 1.2-1) [20]. This peptide is found to depolarize the membrane potential by the efflux of $\mathrm{K}^{+}$ions [21]. These peptides were also found to be membrane selective and very high concentration of $1 \mathrm{mg} / \mathrm{ml}$ was needed to lyse erythrocytes [22].

Alpha helical antimicrobial peptides are also characterized by the presence of kinks or hinge [17]. Cecropins, isolated from hemolymph of diptera insects falls under this category [23]. These peptides were also found to be mostly active against Gram negative bacteria and non hemolytic at MIC concentrations. Cecropins adopt alpha helix at N-terminal and C-terminal separated by a flexible hinge [18]. Melittin, extracted from bee venom, exhibits broad spectrum of activity against bacteria [18]. But this 26-residue peptide is found to be highly hemolytic. Melittin was found to acquire tetrameric helical conformation in crystal form. The monomers of melittin were alpha helical with T11 and G12 forming a kink supporting helices at N and Cterminus. This kink binds together to form a tetramer with hydrophilic residues exposed and hydrophobic buried [24]. When this kink was deleted, the peptide retained antimicrobial activity with reduced hemolytic activity [25].

Helical hairpins are another important structural class under alpha helical peptides. Pardaxin, isolated from fish and MSI-594, a hybrid peptide of MSI-78 and melittin are examples of helical hairpins (Figure 1.2-1). Solution NMR studies of these two peptides in LPS micelles revealed that the peptides adopt helical conformations at $\mathrm{N}$ and $\mathrm{C}$ terminus with a bend forming helical hairpins. Both these peptides also found to exhibit broad spectrum of activity [26, 27].

\subsubsection{Beta sheet antimicrobial peptides:}

Beta sheets stabilized with 2 or more disulfide bridges occupy the next prominent motif for biologically active peptides. These peptides fold into beta sheets in aqueous environment itself with the help of disulfide bridges that results in less entropy loss [17].

Defensins are group of mini proteins isolated from polymorpho neutrophils of mammals, insects and plants [14]. They are rich in cystiene and arginine residues [28]. Defensins are also characterized by the presence of higher number of disulfide bridges that help them to stabilize in membrane environments [29] (Figure 1.2-1). Defensins 
fold into conserved three anti-parallel beta strands with three disulphide bridges and a short helix [30]. In humans alone as much as 34 beta defensins have been isolated and characterized. Among the beta defensins, HBD-3 is known to be active even at physiological salt concentrations [31].

Tachyplesins are another group of beta sheet AMPs derived from horse shoe crab hemocytes. They are so unique that the disulphide bonding pattern is conserved among all members of tachyplesins [22]. Cysteine deleted linear analog of tachyplesin was also found to acquire beta hairpin structure [32]. It was found to abolish hemolytic activity with retained antimicrobial and LPS binding activity [33]. On the other hand Protegrins, derived from porcine leucocytes, loses cell selectivity and salt tolerant antimicrobial activity upon mutation or deletion of cysteines [34] (Figure $1.2-1)$.

\subsubsection{Extended and Loop structures of AMPs:}

Apart from helical and beta sheet motifs, AMPs also adopt extended or loop conformations with broad spectrum activity. Such peptides were found to be rich in one or more specific aminoacids. Indolicidins are derived from bovine neutrophils and are found to be rich in tryptophan residues. It is found to be membrane active but acquire extended conformation even after membrane interaction (Figure 1.2-1). Tritripticin, a cathelicin derived peptide, also rich in Trp residue adopt extended conformation [35].

Thanatin is an insect defense peptide known to adopt loop structure with a disulphide bridge (Figure 1.2-1). It is found to be bactericidal and fungicidal [36]. Gramicidin A is a, channel forming peptide. CD studies suggest that the peptide adopt parallel and antiparallel intertwined double helices [37]. X-ray crystallography studies of Gramicidin/cesium crystals suggested that the peptide folds into double helical dimer (Figure 1.2-1).

\subsection{Mode of action of AMPs:}

The mechanism of action of antimicrobial peptides is so unique involving outer and cytoplasmic membranes. Since the permeabilization of membranes does not require 
any receptors, possibility of development of resistance is likely to be uncommon [38]. At low peptide/lipid ratio, AMPs lie parallel to the membrane. After reaching threshold concentration or higher peptide/lipid ratio, AMPs may aggregate or insert perpendicular to the lipid bilayer and exert killing mechanism [39]. Three common modes of action of AMPs include (a) Barrel-stave pores, (b) Toroidal pores and (c) Carpet mechanism (Figure 1.3-1).

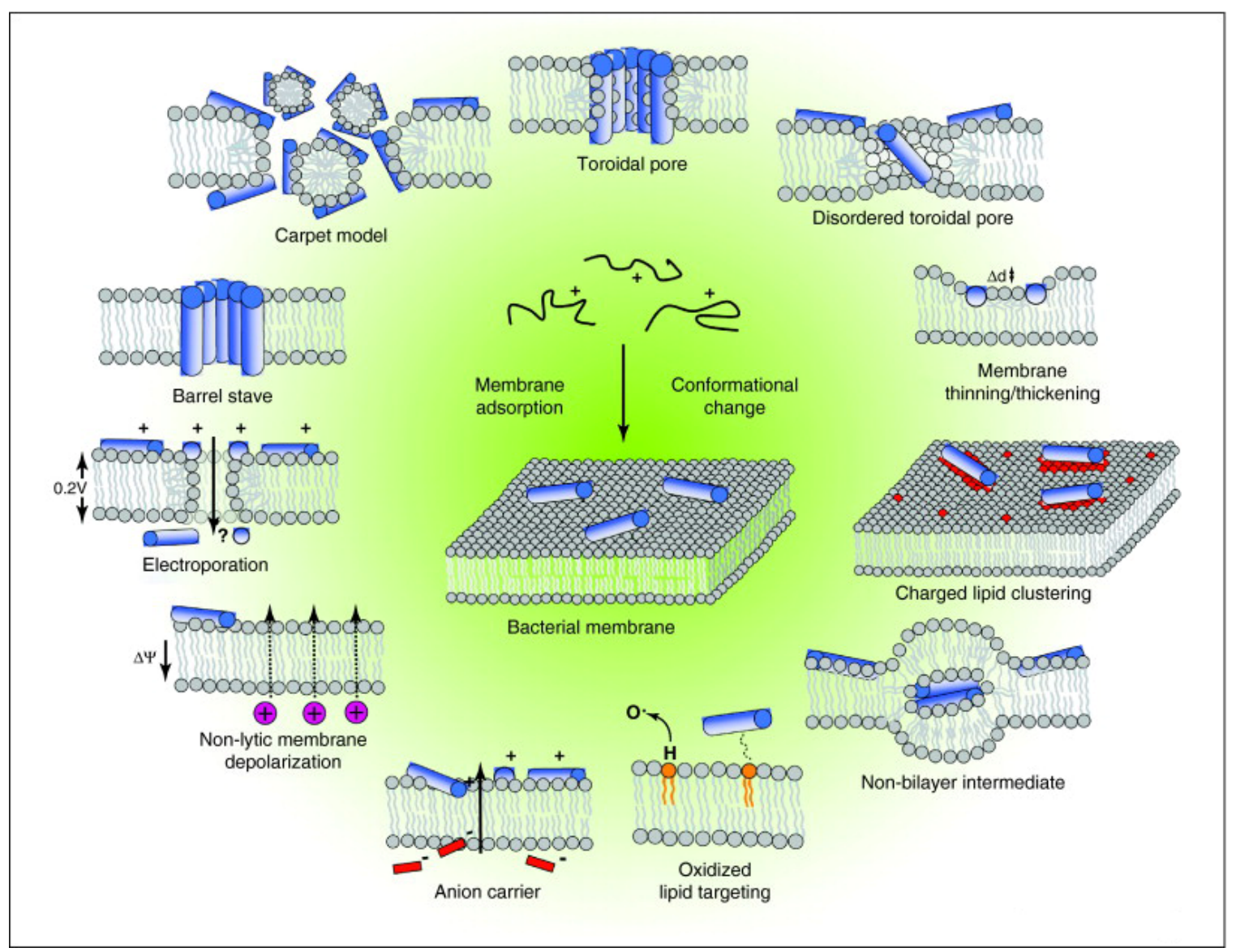

\section{Figure 1.3-1: Different modes of action of AMPs. Taken from ref [14].}

Upon membrane interaction, peptides that adopt alpha helices tend to form pores. In the barrel-stave pores, alpha helices oligomerize and form transmembrane bundle that inserts perpendicularly into the bilayer. The hydrophobic core of the amphipathic helix interacts with hydrophobic lipid core while the hydrophilic part forms the lumen of the barrel [38]. Long peptide helices are needed to insert completely into the bilayer structure. Alamethicin is known to form ion pore channels and adapt this barrel-stave mechanism [38]. 
After acquiring alpha helical conformation, some peptides like magainin, LL-37 form pores lined with helices perpendicularly inserted in to bilayer [38]. Unlike barrel stave pores, in toroidal pores, the polar residues of the peptides interacts with phospholipid head group of the bilayer [14]. Molecular dynamics simulation studies of magainin analog peptide revealed a disordered toroidal pore model where only one peptide helix was found in the center of the pore (Figure 1.3-1). Other peptide helices lie parallel to the bilayer [39].

Most of the antimicrobial peptides were found to act via carpet or detergent like mechanism. Irrespective of the length, conformation or orientation of the peptide, carpet mechanism explains mode of action of AMPs before the formation of pores [40]. The cationic aminoacid residues of the peptides are electrostatically attracted towards the negatively charged phospholipid bilayer thereby inducing micellization of the membrane [38]. When the peptide reaches threshold concentration, toroidal pores are formed (Figure 1.3-1).

Apart from these three classical mechanisms of killing, some peptides act through alternative mechanisms like oxidized lipid targeting, electroporation, membrane depolarization etc [14] (Figure 1.3-1). All of these mechanisms explain the mode of action of AMPs that acquire alpha helical conformations. The beta sheet peptides that are stabilized with disulphide bridges acquire this conformation in aqueous solution itself and hence upon membrane interaction, gets stabilized and exert its killing activity through toroidal pores [41].

It has also been found that membranes are not the only active targets for AMPs. Nucleic acids has been the target for indolicidins where it was found to interfere with DNA synthesis [42]. Buforin II localizes in the cytoplasm by passing through the cytoplasmic membrane without completely destroying it [43].

\subsection{Membrane composition:}

Eventhough the target of AMPs are membranes rather than specific receptors, it can specifically target bacterial membranes when compared to mammalian membranes. Bacterial membranes are rich in negatively charged lipids like cardiolipin, phosphatidyl glycerol and asymmetric LPS (Table 1.4-1). 
Bacteria can be categorized into Gram negative and Gram positive bacteria depending on its membrane composition. Gram negative bacteria are characterized by the presence of thick outer membrane followed by a periplasmic space, peptidoglycan with periplasmic space and inner membrane (Figure 1.4-1). The outer membrane of Gram negative bacteria is indeed made up of two leaflets; asymmetric LPS on the outer leaflet and PE on the inner leaflet [44]. On the other hand, Gram positive bacteria have peptidoglycan layer followed by periplasmic space and inner membrane (Figure 1.4-1).

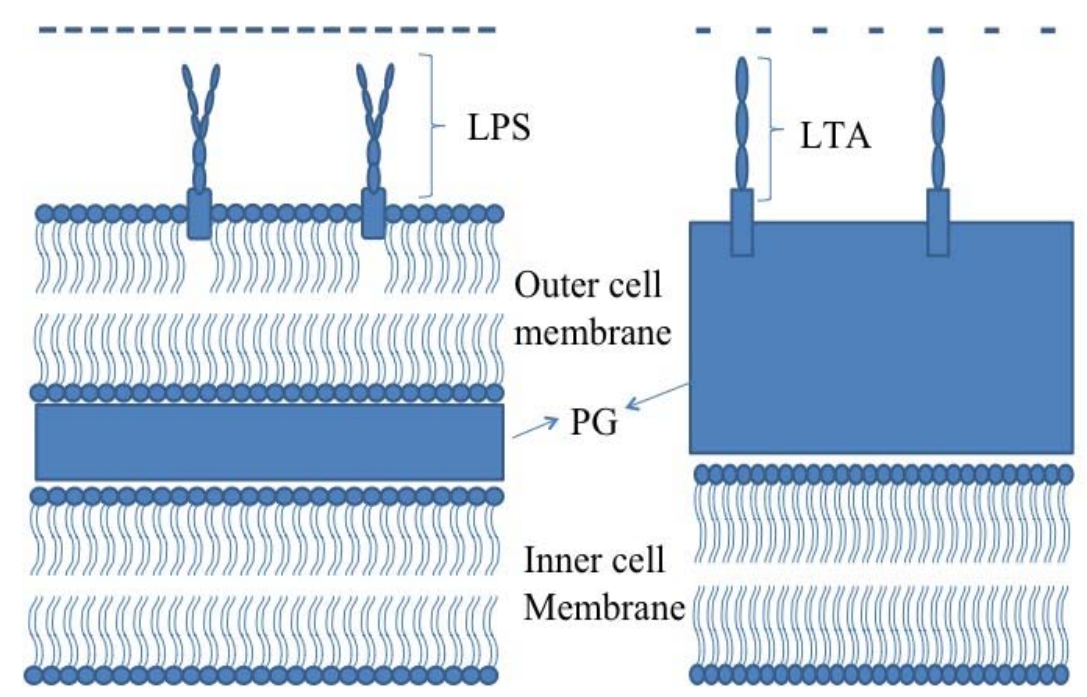

Figure 1.4-1: Interpretation of Gram negative and Gram positive cell wall and its respective charges.

Mammalian membranes are in turn made up of zwitterionic phospholipids like phosphatidylcholine and has cholesterol in both inner and outer membrane [45] (Table 1.4-1). The presence of cholesterol adds extra strength to the bilayer that it cannot bend or tilt upon AMP binding [46]. 
Table 1.4-1: Composition of different lipids in the membrane of bacteria and mammalian cells (PE-Phosphatidyl ethanolamine, LPS- Lipopolysaccharide, LTA- Lipoteichoic acid, PG-Phosphatidyl glycerol, PC-Phosphatidylcholine, PSPhosphatidyl serine).

\begin{tabular}{|l|l|l|l|}
\hline $\begin{array}{l}\text { Membran } \\
\text { e type }\end{array}$ & \multicolumn{2}{|l|}{ Bacteria } & Mammals \\
\cline { 2 - 4 } & Gram negative & $\begin{array}{l}\text { Gram } \\
\text { positive }\end{array}$ & \\
\hline $\begin{array}{l}\text { Outer } \\
\text { membrane }\end{array}$ & PE+LPS+cardiolipi & LTA & $\begin{array}{l}\text { Cholesterol+sphingomyelin+PC+P } \\
\text { E }\end{array}$ \\
\hline $\begin{array}{l}\text { Inner } \\
\text { membrane }\end{array}$ & PE+cardiolipin+PG & $\begin{array}{l}\text { LysoPG+P } \\
\text { G }\end{array}$ & PE+PC+sphingomyelin+PS \\
\hline
\end{tabular}

\subsection{Membrane lipids and curvature strains - an insight into membrane permeation and pore formation:}

Membranes are the protective covering of the cellular components and also act as barrier for the permeation of compounds in and out of the cell. Apart from specific folding of AMPs to be membrane active, permeation of AMPs across membrane highly depends on membrane lipid composition also [47]. There exists polymorphism between the lipids present in the membrane. It was found that the lipids in the bilayer state can aggregate to form non lamellar state under extreme conditions [48]. One such extreme condition were the stress imposed by the binding of AMPs that induce lamellar phase lipids to form non lamellar phases [49]. Lamellar lipid phase (L $\alpha)$ i.e. arrangement of lipids in bilayer phase is considered to be biologically relevant stabilized structure. In this phase, the acyl chains are in melted form that allows dynamic movement of lipids [49]. Induction of curvature stress in this lamellar phase will destabilize the membrane [50]. 

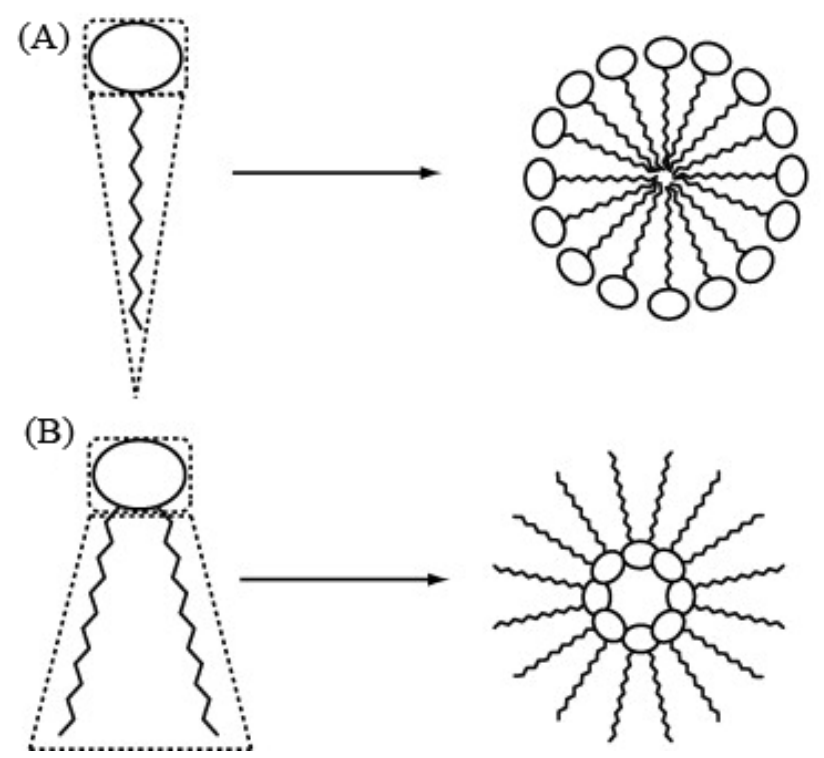

\section{Figure 1.5-1: (A) Positive curvature strain or $H_{I}$ phase and (B) negative curvature strain or $H_{I I}$ phase. Taken from ref [49].}

The non lamellar lipids phases can be categorized into two types depending on the curvature of the head groups and acyl chains it adopts upon stress.

* If the head group area exceeds the area occupied by the acyl chains, micellization results. This is indicated as $\mathrm{H}_{\mathrm{I}}$ phase or positive curvature strain (Figure 1.5-1).

* If the area occupied by head group is smaller than the acyl chains, then the lipids assume inverted cone shape. This is indicated as $\mathrm{H}_{\text {II }}$ phase or negative curvature strain (Figure 1.5-1).

Transition temperature of lipids can be defined as the temperature that brings about critical change in phase of the lipids. $\mathrm{L} \alpha-\mathrm{H}_{\mathrm{II}}$ transition temperature is generally taken as a measure to determine the type of curvature strain imposed by AMPs upon membrane interaction thereby recognizing the mode of action of AMPs [49]. Positive curvature strain is accompanied by increase in $\mathrm{L} \alpha-\mathrm{H}_{\mathrm{II}}$ transition temperature and negative curvature, by decrease in $\mathrm{L} \alpha-\mathrm{H}_{\mathrm{II}}$ transition temperature. Studies on AMPs like Magainin, MSI78 that forms toroidal pores are found to impart positive curvature strain of the lipids in the membrane [51]. The formation of toroidal pores require the fusion of both inner and outer membrane lipids that result in continuous bending of 
the membrane [38]. This fusion is brought about by the appropriate interaction of charged residues of the peptide with polar head group of lipids and hydrophobic residues of AMPs buried in hydrophobic core of lipids [49].

The AMPs like buforin-II, Polyphemusin-1 that localizes in the cytoplasm by penetrating through outer membrane exhibit negative curvature strain. These peptides exhibit broad spectrum of excellent antimicrobial activity accompanied with reduced calcein leakage from liposome models and decrased $\mathrm{L} \alpha-\mathrm{H}_{\mathrm{II}}$ transition temperature $[52]$.

\subsection{Cell selectivity of AMPs and role of cholesterol:}

The most fascinating property of some antimicrobial peptides is their cell selectivity. AMPs like Cecropins, Magainins are selectively active towards bacteria while peptides like Melittin, Gramicidins affect both bacteria and normal cells [53]. The cell selectivity is mainly based on composition of different membranes. Mammalian cell membranes are rich in zwitterionic phospholipids in outer leaflet and cholesterol in both outer and inner leaflets. Cholesterol is completely absent in bacterial membrane [53]. The bacterial membrane exhibit a net negative charge because of the presence of anionic phsopholipids that electrostatically attract the positively charged AMPs [54]. Cholesterol is found to decrease the area that is occupied by phospholipids that results in increase in rigiditiy of the membrane [55]. This reduces the membrane interacting ability of AMPs (Figure 1.6-1).

Bacterial membranes also contain zwitterionic PE (phosphatidylethanolamine) in both leaflets of outermembrane (Table 1.4-1), yet susceptible to action of AMPs. The probable reason could be that AMPs like Magainins induce positive curvature strain on bacterial membrane that imparts bending and fusion of phospholipids with peptide helices resulting in the formation of toroidal pores. Whereas in mammalian membranes, the presence of cholesterol prevent such bending [46]. The high hydrophobic content of peptides like Melittin and Gramicidin has been found to be the reason behind their cytotoxicities. In Melittin, a leucine zipper like motif has been found to be responsible for its high hemolytic activity, mutation of which reduced hemolytic activity by retaining antimicrobial activity [56]. In another study, deletion 
of residues in the hinge region of Melittin retained antimicrobial activity with reduced hemolytic activity [57].
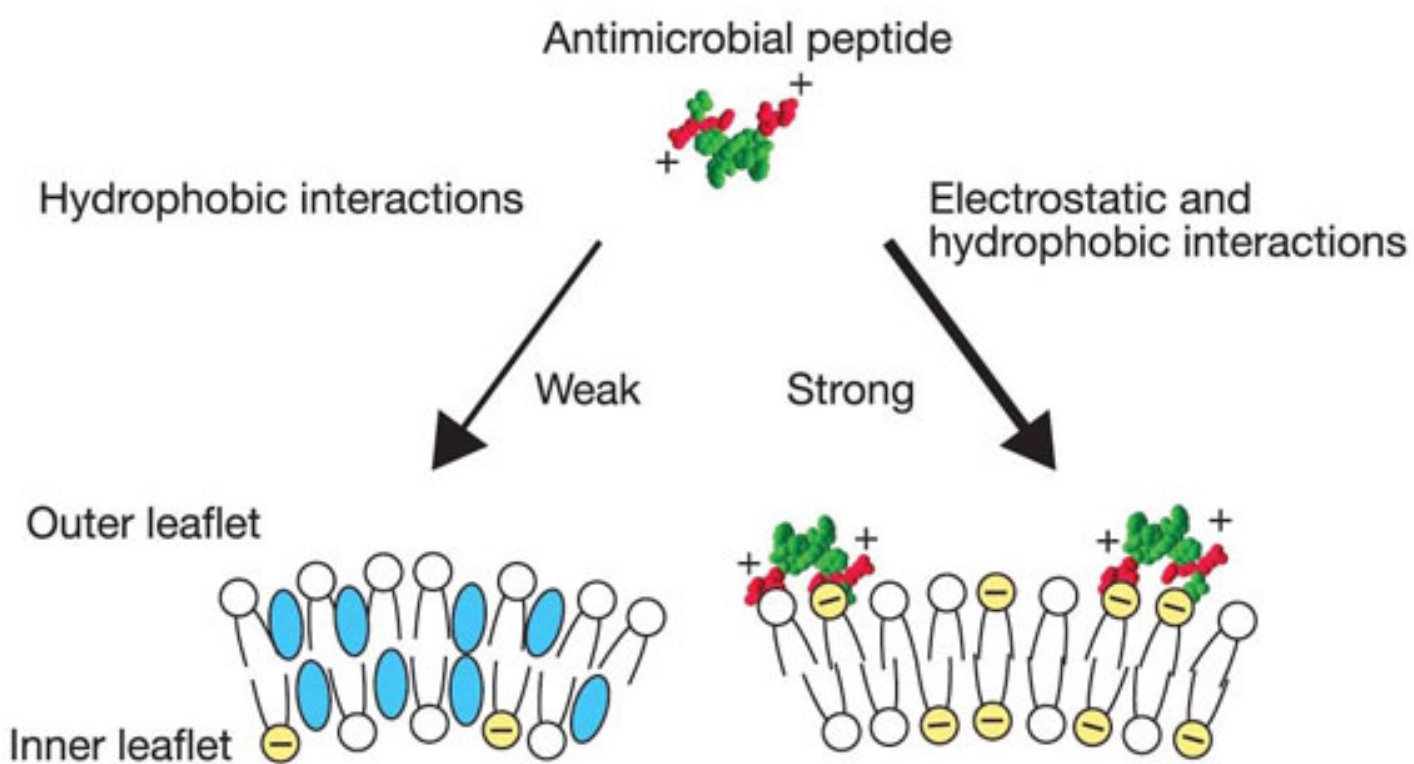

Prototypic plasma membrane of a Bacterial cytoplasmic membrane multicellular animal (erythrocyte)
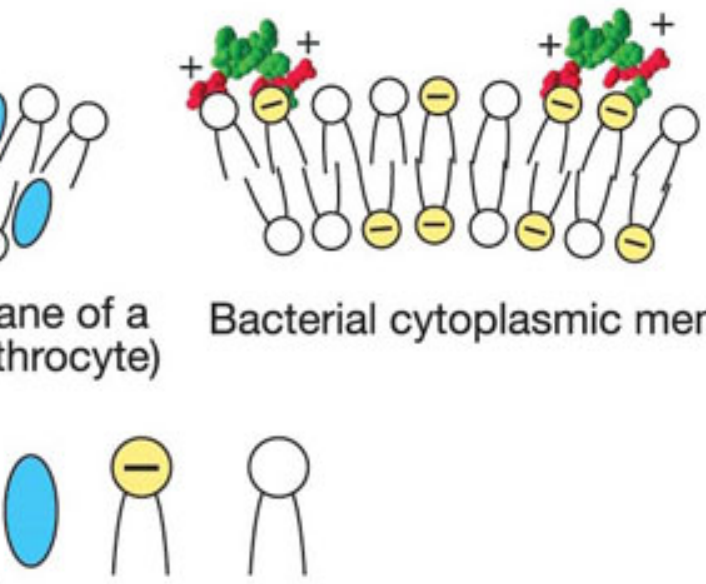

Cholesterol

Acidic phospholipids

Zwitterionic phospholipids

Figure 1.6-1: Mechanism of cell selectivity by AMPs: Net positively charged AMPs are attracted towards anionic bacterial membrane followed by hydrophobic interactions and membrane disruption. Whereas weak hydrophobic interactions govern the binding of AMPs to mammalian membranes and presence of cholesterol further hinders toxicity. Taken from ref [58].

Studies of Gramicidins also demonstrated that reduction of hydrophobicity of the peptide lead to reduced hemolytic activity [59]. It has also been postulated that aminoacid residues need not to be inserted into hydrophobic core of the membrane for bacterial membrane disruption [60]. Rather the interaction of hydrophobic residues of 
the peptide with hydrophobic core of the lipids in lipid-water interface is sufficient of antimicrobial action [61].

Taken together all these results suggest those AMPs that have appropriate propotion of basic and hydrophobic residues can selectively kill bacterial cells and exhibit reduced toxicity to mammalian cells.

\subsection{Bacterial LPS and Sepsis:}

Bacteria produce two types of toxins, exotoxins that are secreted by bacteria and endotoxins where any cell component of bacteria acts as toxin eliciting immune response. The pathogenicity of Gram negative bacteria stems from LPS present in the outer layer of outer membrane. LPS is an endotoxin that is responsible for the activation of innate immune response in host cells. LPS is in turn divided into three regions namely the endotoxic principle - LipidA, non repeating units of core oligosaccharides and n-repeats of O-antigen (Figure 1.7-1).

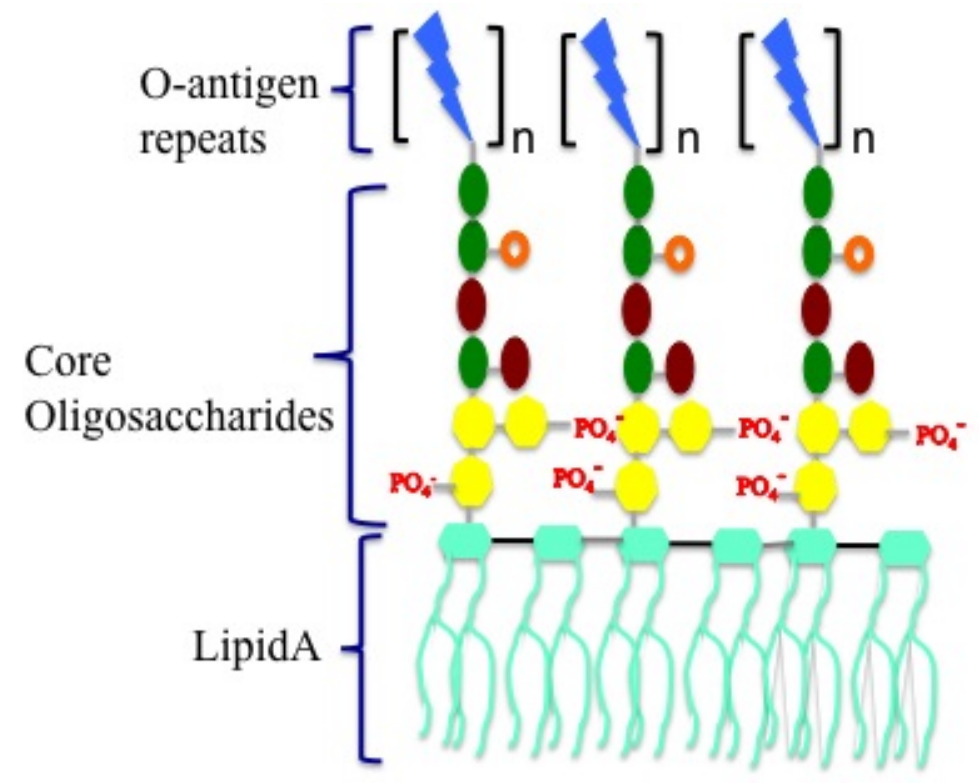

Figure 1.7-1: Structure of Lipopolysaccharide: LPS is highly asymmetric that it contains three well defined regions namely (a) O-antigen repeats of approximately 40 units, (b) core oligosaccharides that contain specialized sugar molecules such as ${\mathrm{KDO}-\mathrm{PO}_{4}}_{4}$ and (c) Lipid A, the endotoxic portion of LPS. Adapted from ref [62]. 
The negative charge on Gram negative bacteria is imparted by anionic sugars (KDO) in core oligosaccharides and additional phosphate groups in LipidA portion of LPS. These anionic groups are held together by divalent cations such as $\mathrm{Mg}^{2+}$, chelation of which results in the disintegration of LPS and subsequently outer membrane [63]. The antibiotic treatment for killing of bacteria releases LPS, which in elevated levels leads to a cascade of events leading to sepsis, endotoxic shock.

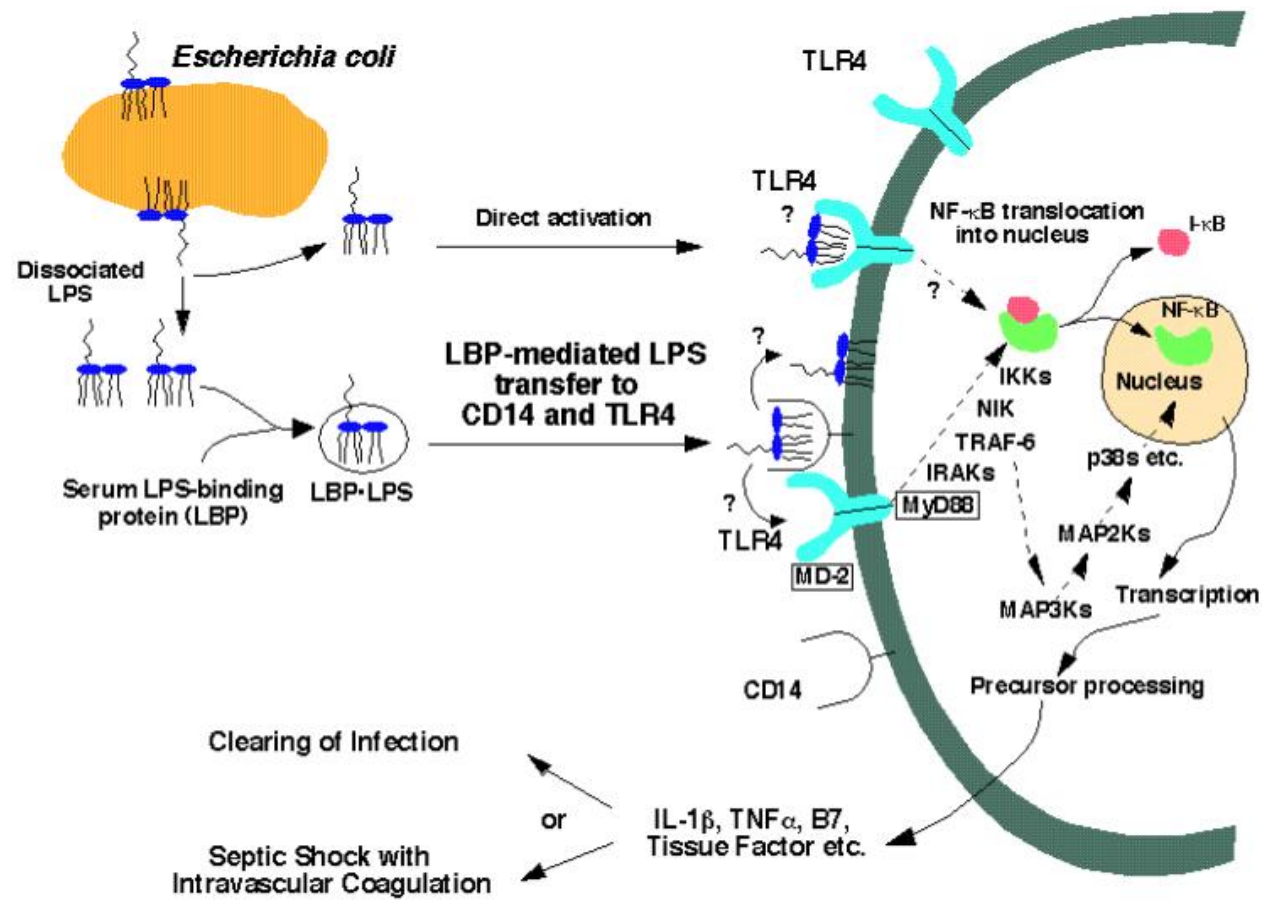

Figure 1.7-2: Sepsis induction pathway: Recognition of free circulating LPS by LBP, CD-14 and subsequently by TLR-4 that mediates a cascade of events leading to sepsis. Taken from ref [64].

Sepsis is an acute medical condition of inflammation resulting from the hyperactivation of innate immune molecules [65]. Sepsis or septicemia also occupies tenth position in the dreadful diseases list and recent epidemiological reviews on sepsis show that there are no significant improvement in the treatment of sepsis [66]. The ability of LPS to initiate sepsis inducing pathway is well formulated $[30,67]$. The killing of pathogenic Gram negative bacteria or its cell division sometimes results in 
the release of LPS (Figure 1.7-2). This circulating LPS molecule is recognized and bound by LBP (LPS binding protein) and CD14. LPS-LBP complex is then transferred to TLR-4 and subsequently to MD-2 (myeloid differentiation protein-2) which in turn initiates the cascade of events that synthesizes inflammatory cytokines in huge amounts resulting in multiple organ failure via sepsis [68] (Figure 1.7-2).

\subsection{LPS as outer membrane permeability barrier:}

Apart from the involvement of LPS in the induction of inflammatory pathway, it also acts as permeability barrier for many of the hydrophobic components [69]. X-ray diffraction analysis of LPS from Salmonella strains revealed that LPS also acquires the bilayer lamellar state as the normal phospholipids in the bilayer arrangement of the outer layer. But the lipidA, the endotoxic principle of LPS acquires higher state of order, where the fatty acyl chains of lipidA are arranged perpendicular to the membrane surface and the sugar backbone is tilted imparting a shed roof like shape [70]. This arrangement of lipidA confers rigidity to LPS in the same way as cholesterol imparting on mammalian membranes [44]. Moreover, the acyl chains of lipidA also differs from normal phospholipids in that all the acyl chains are saturated and some are hyroxy derivatives of fatty acids [69]. It was also envisioned that the divalent cations form tight cross bridges with adjacent LPS molecules forming a rigid structure [71].

Besides this rigid packing of lipidA, heterogeneity of LPS molecules also found to act as an important contributing factor towards its barrier property. LPS is also called as glycolipid where the polysaccharide region is made up of $\mathrm{O}$-antigen and core polysaccharides and lipid for lipidA region. The polysaccharide region is mainly required for the survival of Gram negative pathogens. The bacteria that have complete LPS molecule produces smooth colonies on agar plates and hence called smooth type mutants (Figure 1.8-1A). On the other hand, the mutants that lack O-antigen are called Rough type and mutants that lack O-antigen and outer core of core oligosaccharides as Deep-rough mutants (Figure 1.8-1A). Mutants that completely lack the polysaccharide region cannot maintain the structural integrity and hence found to be not viable [64]. 
(A)

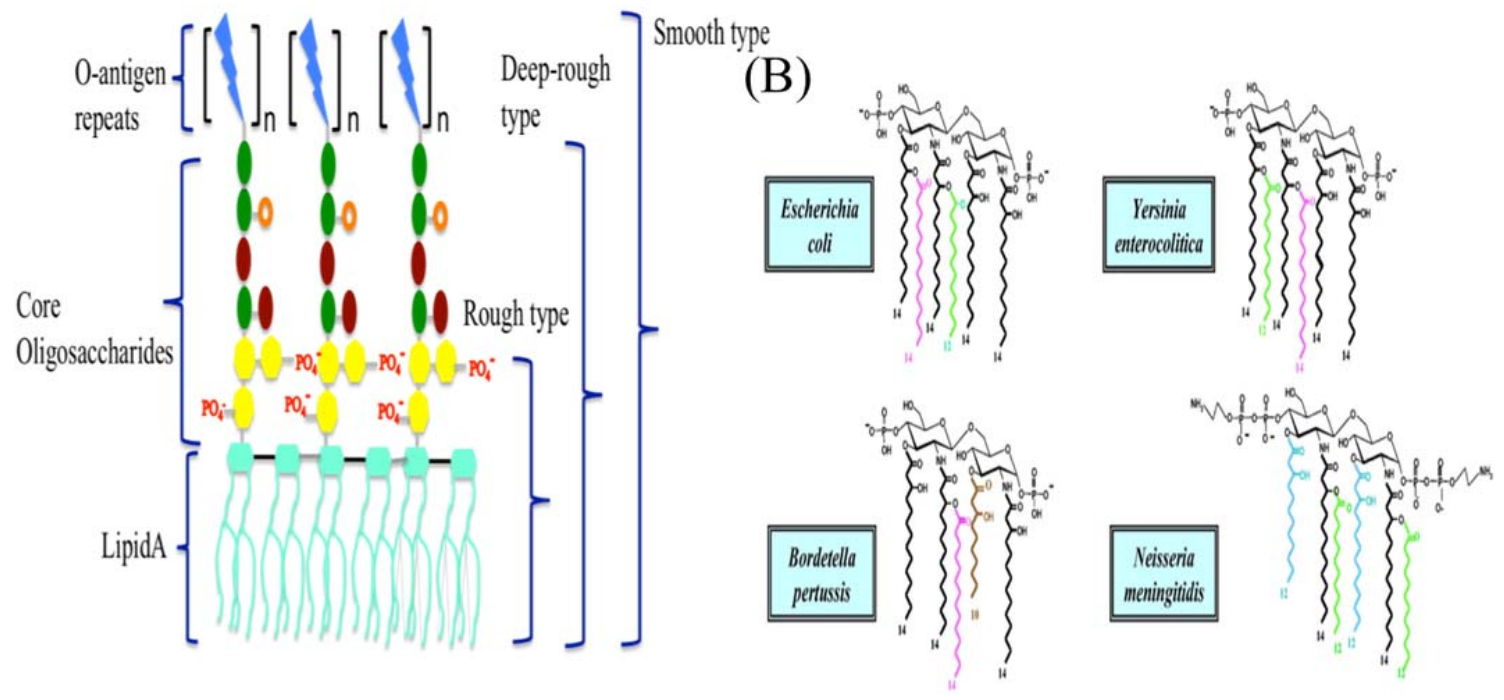

Figure 1.8-1: Heterogeneity of LPS: (A) Mutations in polysaccharide regions of LPS appears to be directly related to structural integrity and survival of the pathogen; Adapted and modified from ref [72], (B) Difference in lipidA acylation for the permeability barrier; Taken from ref [73].

Additionally the heterogeneity of LPS molecules also arise from different degree of fatty acid acylation (Figure 1.8-1, B). Such defects generally arise from the mutations in fatty acyl transferases during post translational modifications [73]. Hence clue for the fact that LPS acts as permeability barrier arises from the careful examination of these mutants. It was found that the mutants that lack phosphate groups in oligosaccharide region (rfaP-) are highly susceptible to novobiocin and chelation of divalent metal ions have been found to increase the susceptibility of deep rough mutants [74]. Epifluorescence micrograph studies of LPS GUVs demonstrated the presence of more lipid - condensed region when compared to PE/PG GUVs which are rich in lipid - expanded region [75]. Such condensed regions act as trap for the passage of highly aggregated structures of AMPs.

\subsection{Antiendotoxic antimicrobial peptides:}

One of the important steps in the execution of killing of bacteria by most antimicrobial peptides is the outer membrane permeabilization. It should also be noted that the 
endotoxin neutralization property and bacterial killing are two different mechanisms and the AMPs that are successful in killing bacteria may not be successful endotoxin neutralizers [76]. To induce the production of cytokines, ciruculating LPS must be recognized by LBP (Lipopolysaccharide binding protein) that flaunts positive charge on its surface and assumes transmembrane configuration [77]. The diphosphorylated lipidA with six fatty acyl chains in asymmetric distribution that assumes conical shape has been found to be the most active form for the activation of immune cells [78]. On the otherhand, the precursor of lipidA, compound 406 that is short of two fattyacyl chains was found to have opposite reaction to lipidA [64]. Studies on compounds like cardiolipin and phosphatidyl glycerol that are structurally similar to compound 406 revealed that the phsopholipids compete with lipidA to bind to LBP and hence interfere with the binding of LPS to LBP [79]. It was also noted that only negatively charged phospholipids competes with LPS to bind to LBP and not the zwitterion lipids [79].

Host defense cationic AMPs that shows remarkable antimicrobial activity has been found to dissociate LPS and its stimulation of inflammatory pathway leading to release of cytokines [80]. Some examples of AMPs from natural sources, which can also act as antiendotoxic agents, are discussed below.

\subsubsection{Bactericidal permeability increasing protein (BPI):}

The polymorphonuclear leucocytes and azurophil granules have been found to be the manufacturing and storing sites for a large number of defense proteins like BPI, BP, CAP57 etc [81]. Bactericidal/permeability increasing (BPI) protein was found to specifically interact with Gram negative bacteria and kill them [81]. It was also found to exhibit about $45 \%$ sequence homology with Lipopolysaccharide binding protein (LBP) [82]. The crystal structure of BPI protein reveals a unique boomerang shape with binding pockets of acyl chains in the middle (Figure 1.9-1). It was revealed that BPI forms three structural elements with barrel $\mathrm{N}$, barrel $\mathrm{C}$ and a central $\beta$-linker. The conserved residues for LBP and BPI were found to be highest in central linker followed by barrel $\mathrm{N}$ and barrel $\mathrm{C}$ [83]. The side chain topology of barrel $\mathrm{N}$ revealed 
that it is composed of lysine residues that is responsible for antibacterial activity and barrel C promoting phagocytosis of bacteria [83] (Figure 1.9-1).

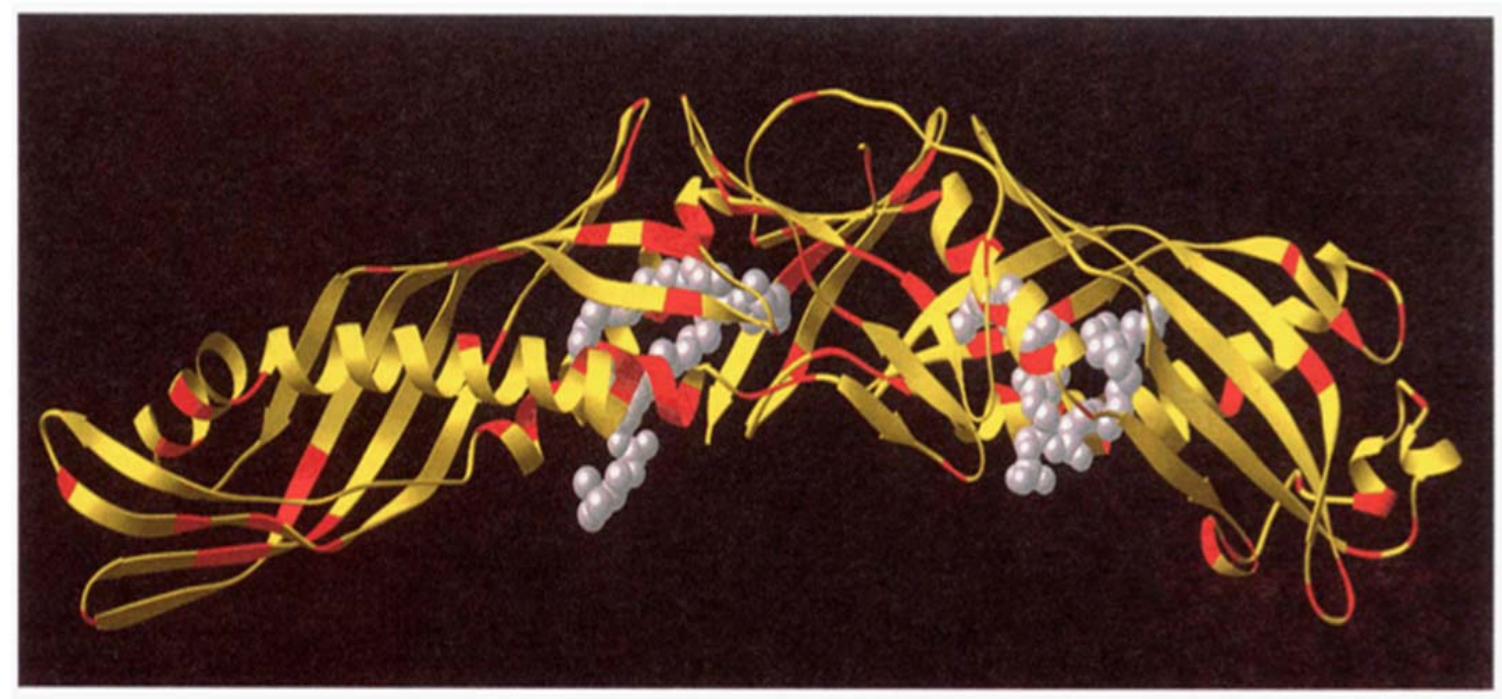

Figure 1.9-1: Crystal structure of BPI: Unique boomerang shape BPI with binding pockets for acyl chain in the middle. The conserved regions for LBP and BPI are highlighted in red color. Taken from ref [83].

Studies on recombinant proteins of N-terminal residues of BPI $\left(\mathrm{rBPI}_{21}\right)$ and LBP $\left(\mathrm{rLBP}_{25}\right)$ clearly revealed the difference between these two mammalian proteins. $\mathrm{rBPI}_{21}$ was found to have antibacterial and anti endotoxic activity which were absent in $\mathrm{rLBP}_{25}$. The LBP were mainly involved in delivering LPS to CD14 whereas BPI proteins were involved in its detoxifying and phagocytosing activities [82]. Sucrose gradient sedimentation analyses of BPI and LBP with LPS revealed that BPI increased sedimentation velocity of LPS by aggregating LPS into larger structures, whereas LBP breaks down aggregates [84] (Figure 1.9-2). 


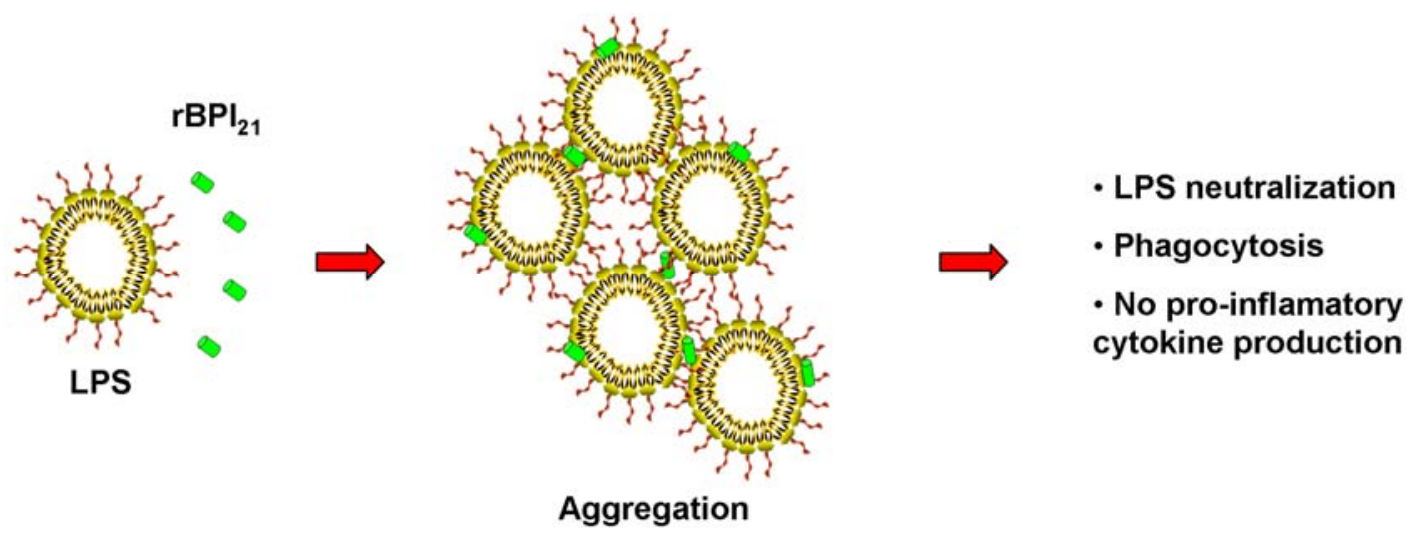

Figure 1.9-2: The N-terminal recombinant rBPI $_{21}$ selectively interacts and aggregates negatively charged LPS thereby bringing about killing of Gram negative bacteria. Taken from ref [85].

Liposome leakage studies of the recombinant protein of N-terminal domain of BPI, $\mathrm{rBPI}_{21}$ disclosed that the protein induces $100 \%$ leakage from negatively charged PG liposomes at concentration as low as $1 \mu \mathrm{M}$. On the other hand, there was little effect on POPC liposomes. Zeta potential studies also revealed that they selectively interact and insert in to hydrophobic environment of anionic phospholipids. These studies explained that the protein mediates the aggregation of negatively charged LPS molecules resulting in the formation of larger aggregates that is easily recognized by phagocytes even before its interaction with LBP [85].

\subsubsection{Polymyxins:}

Polymyxins are lipopeptides identified as secondary metabolites in Bacillus polymyxa [86]. All polymyxins share the same structure except that Polymyxin B (PMB) has DPhe and Polymyxin E (PME) has D-Leu in position 6 of cationic ring (marked as R in Figure 1.9-3). Polymyxins were also found to be active against Gram negative pathogens and the Gram positive bacilli were mostly resistant [87]. The mode of action of polymyxins involves outer membrane permeability and found to specifically interact with lipidA portion of LPS [86]. Interaction studies of PMB with E. coli lipid extracts and red blood cells indicate that the peptide selectively interacts only with $E$. coli lipid extract. The selectivity was found to be based on the interaction of polar head groups of lipids in different membrane [87]. PME was also found to disrupt 
bacterial membranes selectively and found to be even active against MDR P.aeruginosa strains [88].

Isothermal titration calorimetric studies of PMB and PME with LPS and lipidA reveal that the reaction is endothermic and entropy driven [89]. Moreover hydrophobic interactions were found to play prominent role in the binding of the peptides with LPS since the stoichiometry of the reaction was much stable [89]. FTIR studies of head group region of LPS in susceptible and resistant mutants disclosed the difference in interaction of PMB. The bands from phosphate and sugar groups of susceptible strains found to decrease in intensity when compared to resistant strains. Thus the steric changes because of the presence of bulky arabinose residues in resistant strains hinders the binding of PMB [90].

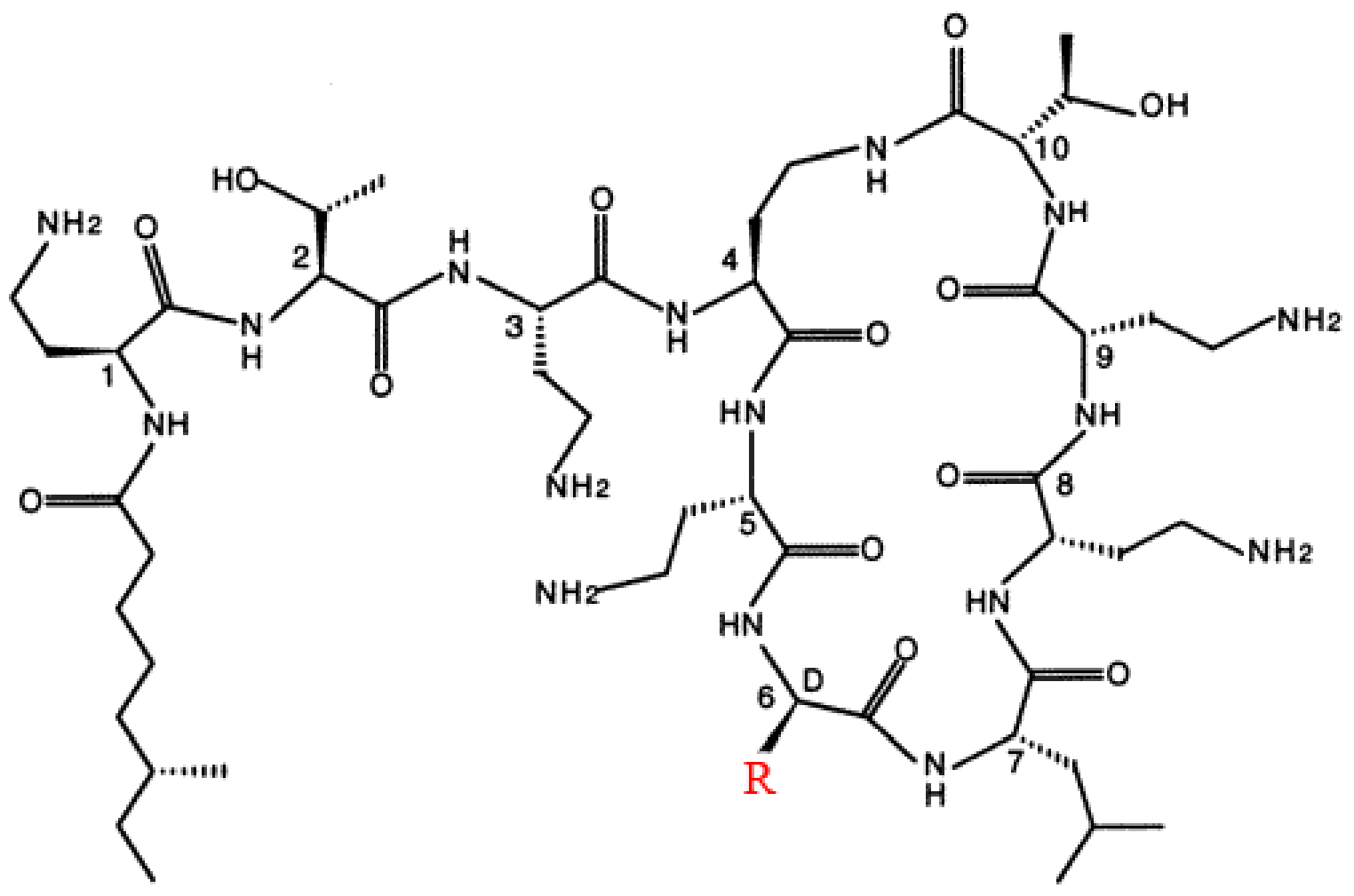

Figure 1.9-3: The basic molecular representation of Polymyxins: A polycationic ring with tripeptide fatty acyl chain. $R$ refers to D-Phe in PMB and D-Leu in PME. Taken from ref [91].

Transfer NOESY NMR experiments on PMB and PME revealed that the peptides adopted amphiphillic conformations where the hydrophobic residues (F6 and L7) were segregated from the cationic Dab (diamino butyric acid) residues forming envelope 
like fold in the ring (Figure 1.9-4, A). Thus the electrostatic interactions between anionic phosphate groups of lipidA and positively charged Dab residues and hydrophobic interaction between residue F6 and L7 (Figure 1.9-4, A) with fatty acyl chains of lipid A explains the entropic mode of action of PMB with LPS [91].

(A)

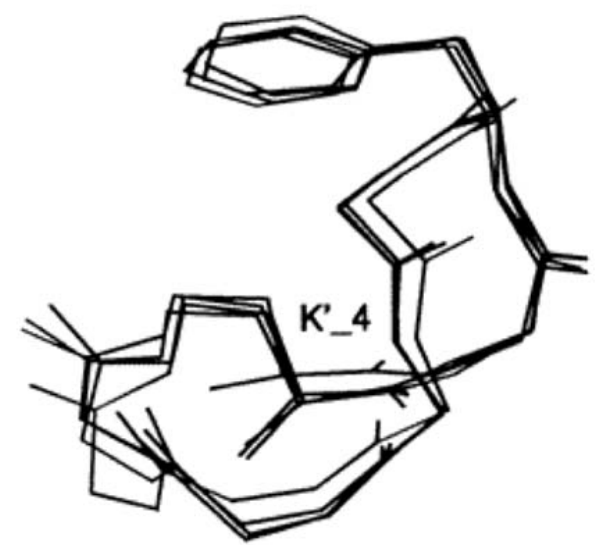

(B)

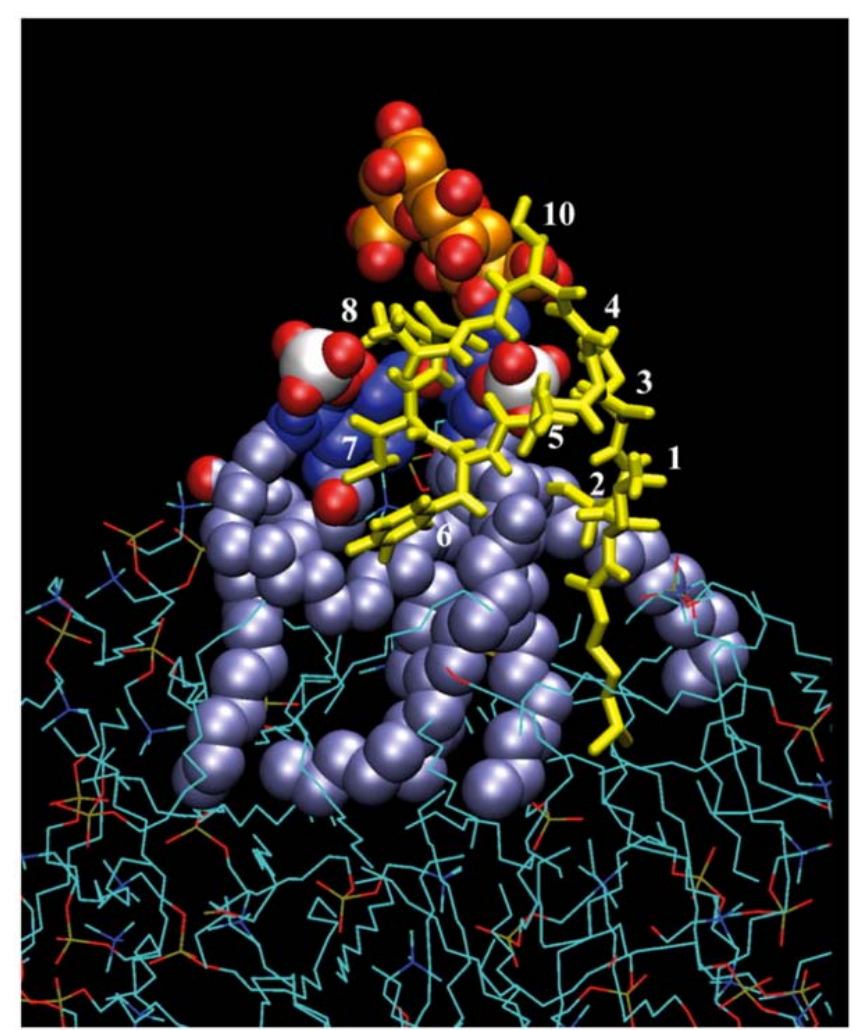

Figure 1.9-4: Three-dimensional structures of PMB in LPS: (A) Overlay of backbone of PMB in complex with LPS derived from distance constraints in trNOESY NMR experiments. Taken from ref [91]. (B) Structure of PMB in complex with LPS in the presence of DPC micelles calculated from Molecular Dynamics. PMB is show in yellow sticks, LPS in spheres and DPC micelles in lines. Taken from ref [92].

NMR studies were also carried out with PMB and LPS complexes in membrane environment. NMR chemical shifts along with MD structure restraints were used for this study, wherein LPS molecules were first inserted into DPC micelles followed by PMB (Figure 1.9-4, B). This study disclosed the interaction of additional hydrophobic binding of residue $\mathrm{L} 7$ to KDO residues (Figure 1.9-4, B). The structure derived clearly 
helped to envision the binding of cyclic Dab residues with phosphate groups of lipidA and residue F6 and L7 with fatty acyl chains of lipid A [92].

\subsubsection{Limulus anti-lipopolysaccharide factor (LALF):}

Limulus anti-LPS factor is originally isolated from horse-shoe crabs and is a small protein known to detoxify LPS in a manner similar to BPI [93]. Crystal structure of LALF revealed that the protein adopts wedge shape with N-terminal helix followed by 4 antiparallel $\beta$-strands and two helices at C-terminal (Figure 1.9-5, A).
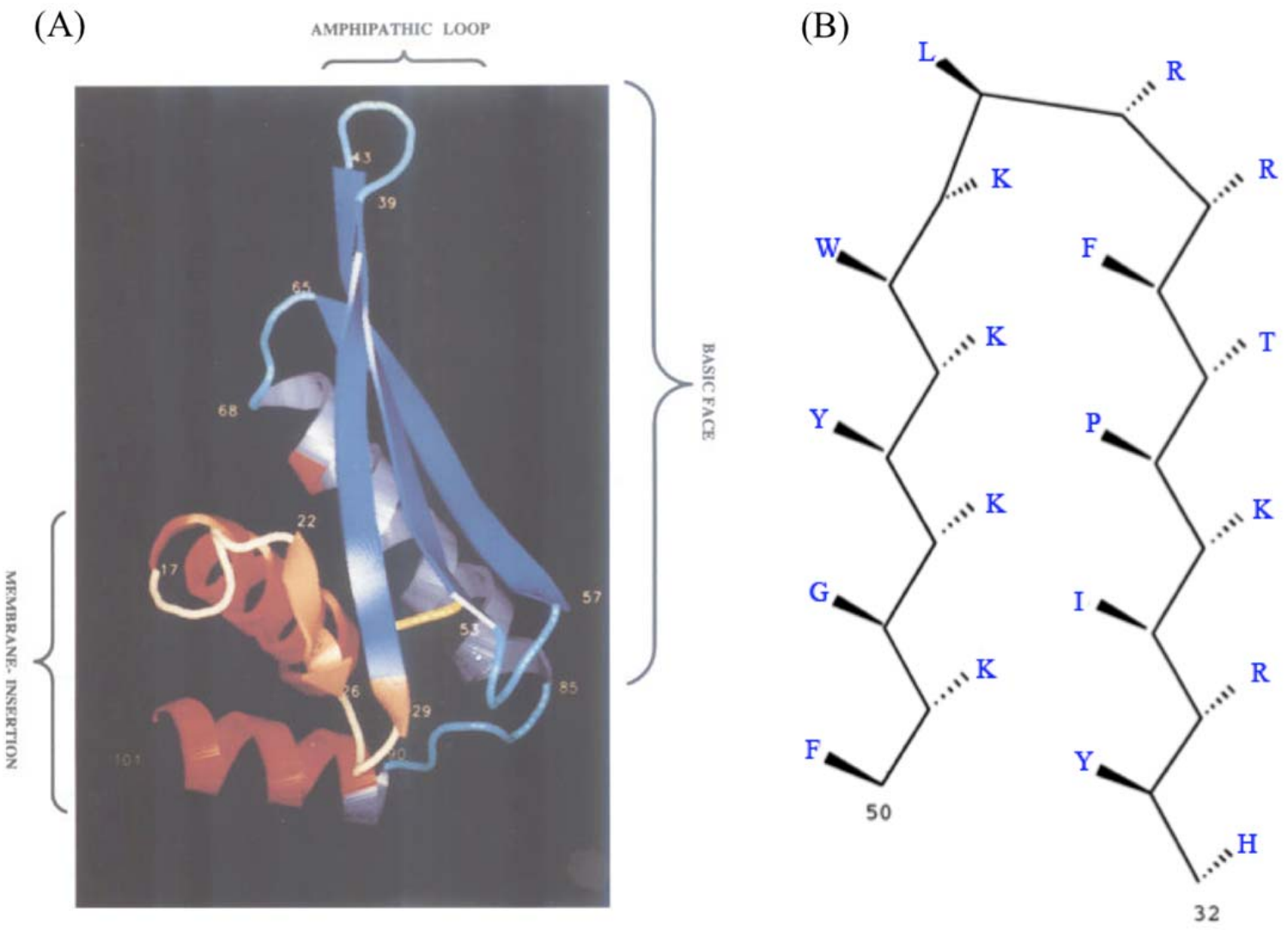

Figure 1.9-5: Crystal structure of LALF: (A) Wedge shaped LALF and (B) Amphipathic loop with aminoacid residues labeled. Taken and modified from ref [94].

The crystal structure also helped to dissect the regions responsible for antimicrobial and antiendotoxic activity. The amphipathic loop was identified for lipidA binding and this loop is found to exhibit $44 \%$ sequence homology with LBP and BPI proteins 
(Figure 1.9-5, B) [94]. Recombinant studies on amphipathic loop, rLALF22 was found to bind to LPS molecules selectively. This loop structure comprises of two $\beta$-strands with hairpin on one end and cyclized with disulfide bond on other end (Figure 1.9-5, B) [93]. Synthesis of overlapping shortened mutants and cyclization of hairpin region alone identified that the cLALF14 was equally active in neutralizing LPS to full length loop [93]. NMR studies of cLALF14 (GCKPTFRRLKWKYKCG) revealed that the central RR residues bind to one of the phosphoryl chain of lipidA while the other chain is bound by lysine residues [95].

Mode of action of cyclized full length loop cLALF22 revealed that the cubic active form of LPS changes to multilamellar inactive forms upon binding [96]. In another study, peptides were synthesized based on the loop sequence. The active peptide was found to interact specifically with LPS by fluidizing the cubic structure of LPS to multilamellar state. But in the case of zwitterion mammalian membrane, the peptide causes rigidification and hence cannot bind [97].

\subsubsection{Cathelicidins:}

Cathelicidins constitute an important class of AMPs that are derived from a wide variety of origins ranging from mammals (humans, monkeys, mice, rabbit, sheep) [98] to non-mammals like Hagfish [99]. The cathelicidins also conserve a cathelin domain at its N-terminus with antibacterial domain at C-terminus [100]. Cathelicidins of chicken origin were named Fowlicidins and three analogues were isolated. Among them, Fowlicidin-1 (RK26) was found to exhibit broad range of antibacterial activity and LPS neutralizing activity [101]. Deletion analog studies of RK26 identified two LPS binding sites residing in V5-P7 and G16-I23. Three dimensional structure determinations of deletion analogs, RG16, LK19 and AK12 in LPS micelles revealed topology of helix binding to LPS (Figure 1.9-6, top panel A, B, C). Docking of these structures disclosed that RG16 and AK12 helices lie parallel to LPS axis whereas LK19 remain diagonally bend [102]. One of the deletion analog, VK22 exhibited antibacterial and LPS binding activity similar to RK26. Atomic resolution structure of VK22 in DPC micelles revealed that the peptide adopts tetrameric structure with 
hydrophobic residues in the center and basic residues at the termini (Figure 1.9-6, top panel D).

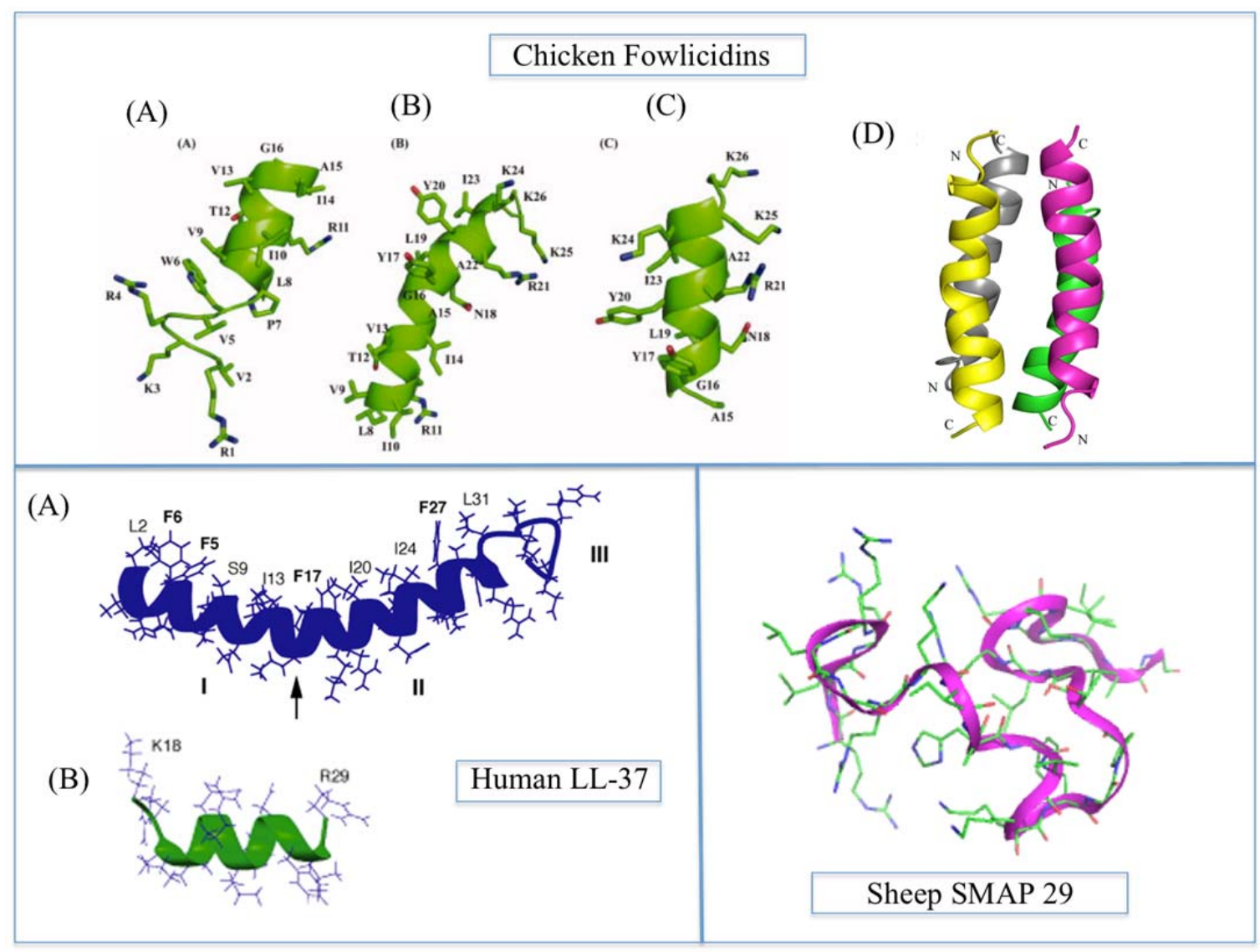

Figure 1.9-6: Cathelicidins from different origin: Top panel: Three dimensional structures of Chicken fowlicidins (A) RG16, (B) LK19, (C) AK12 in complex with LPS (taken from ref [102]) and (D) VK22 in DPC micelles (taken from ref [100]), Bottom panel: Only known human cathelicidin LL37 in SDS micelles and its short active fragment KR12 in D8PG micelles (taken from ref [103]) and sheep SMAP-29 in TFE buffer (modified from PDB ID: 1FRY, ref [104]).

Human cationic antimicrobial protein (hCAP18) releases the only human cathelicidin LL-37 upon bacterial infection [105]. Solid-state NMR studies has revealed that the amphipathic structure of LL-37 interacts in the bilayer interface region and induces disorder in the lipid acyl region [106]. LL-37 is found to oligomerize in zwitterionic membranes but appear as monomers in anionic phosphatidylglycerol lipids. This 
effective membrane selective mechanism has made it a suitable therapeutic agent for further characterization (Figure 1.9-6, bottom panel A). Atomic resolution structure of LL37 in SDS micelles revealed complete helix structure with slight bend at residue 14-16 [103]. This structure divides LL-37 helix into three parts namely, the Nterminal helix that is found to be responsible for chemotaxis and hemolytic activity while C-terminal that is found to be flexible in anionic lipids and the middle bend [103]. Synthetic peptides were designed with various lengths after the bend. Among the peptides, KR12 were found to be highly active and found to adopt helical conformation in D8PG micelles [103] (Figure 1.9-6, bottom panel B).

SMAP-29 is broad spectrum antimicrobial peptide isolated from sheep leucocytes (Figure 1.9-6, bottom panel) [107]. Analysis of structure of SMAP-29 in TFE revealed two LPS binding site at each terminal. The highly cationic N-terminal was found to interact with phosphate groups of lipidA, deletion of which resulted in annulled LPS activity [104].

\subsubsection{Short antiendotoxic peptides:}

The mode of endotoxic binding and LPS neutralization of BPI, Polymyxins and LALF were revealed based on its three dimensional structures. Certain peptide scaffolds were identified and synthetic peptides were generated using such scaffolds as templates [30]. Several other peptides from natural sources were also found to exhibit potent antimicrobial and antiendotoxic activity.

Melittin from bee venom was found to exhibit broad spectrum of activity. The threedimensional structure of Melittin in complex with LPS revealed that it delineates alpha helix at its C-terminus and its entire N-terminal remains flexible (Figure 1.9-7, A). STD-NMR experiments mapped that the residues in C-terminal helix are in close contact with LPS [25].

Pardaxin Pa4, derived from fishes are efficient pore forming AMP and is 33 aminoacids long (Figure 1.9-7, B). Structure determination of Pa4 in complex with LPS showed that the peptide adopts horse - shoe shaped helical structure. STD-NMR experiments reveal that the residues $\mathrm{K} 8$ and $\mathrm{K} 16$ are in close proximity can 
electrostatically attract the lipidA with S11 and T17 supporting them (Figure 1.9-7, B) [27].

(A)

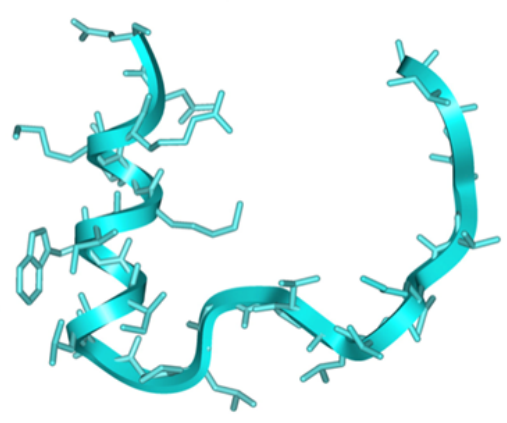

(B)

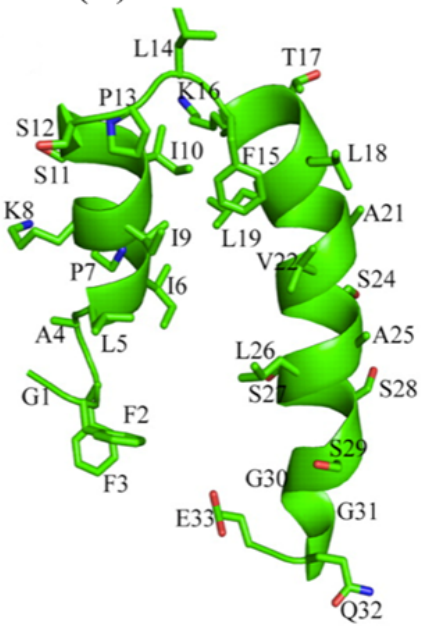

(C)

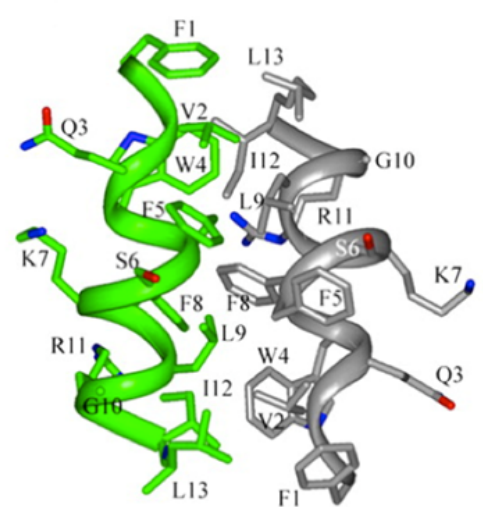

Figure 1.9-7: Three dimensional solution state NMR structures of short peptides, (A) Melittin, (B) Pardaxin and (C) Temporin L, in complex with LPS micelles. Taken from ref [25, 27, 108].

Temporins are short AMPs isolated from frog and are found to be potent bacterial sterilizers even though the net positive charge can range from none to three [109]. Among them, TemporinL (TL) was found to be independently active and Temporin A and Temporin B are synergistically active with TL. The aggregation of TB and TA into higher order oligomers was found to be the reason behind its inactiveness. Analysis of tr-NOESY NMR spectra of TL in LPS bound form revealed that the peptide adopts antiparallel dimer conformation in a basket shape (Figure 1.9-7, C). This segregates the cationic and hydrophobic residues on opposite sides of the basket shape exhibiting amphipathicity required for bacterial killing [108].

\subsection{Strategies for the design of antimicrobial and antiendotoxic peptides:}

The peptides from natural sources that show wide range of antimicrobial activity may not be suitable for pharmaceutical development [110]. In spite of its broad spectrum 
activity, AMPs suffer from several drawbacks that includes high cost, susceptible to proteases and inactivation at physiological salt concentration [111]. Template based design is one of the widely accepted strategy for the development of antimicrobial, non-hemolytic peptides [112]. AMPs from natural sources serve as templates for the design of bacterial selective peptides with modifications such as N-terminal acylation [113], inclusion of d-enantiomers of aminoacid residues for protease stability [114], increase in hydrophobic surface for salt resistance [115], chimerisation of active peptide scaffolds [116], endtagging of AMPs [117], inclusion on non-natural aminoacids [118] etc (Figure 1.10-1).

\section{End-tagging}

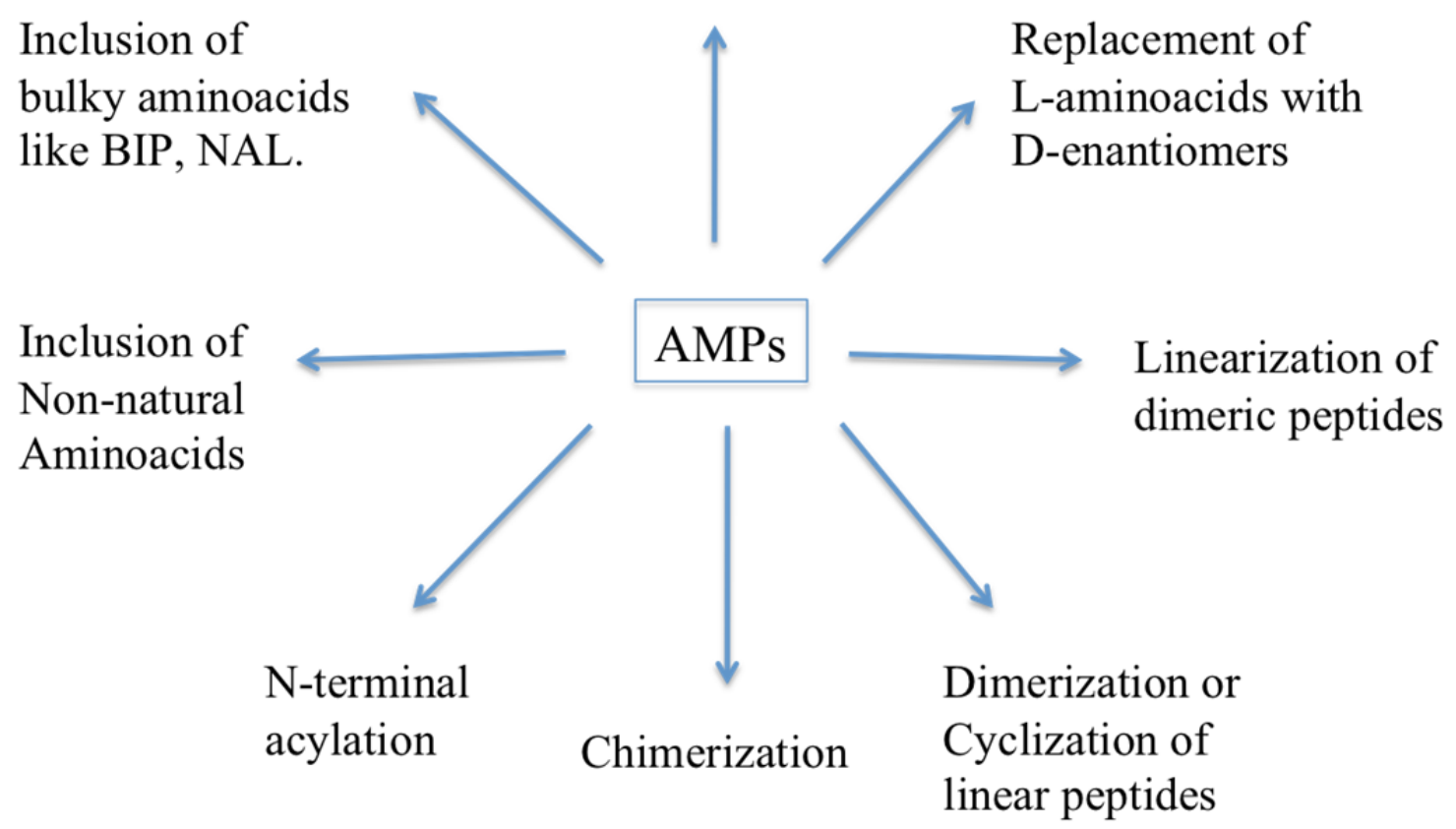

Figure 1.10-1: Different strategies that are used to design antimicrobial peptides to enhance bacterial selectivity, salt tolerance and protease resistance.

\subsubsection{Lipopeptides - N-terminal acylation of AMPs:}

Addition of fatty acyl chains of different chain lengths to AMPs shows increased microbial sterilization probably by increasing incorporation of peptides deeply into the membrane [119]. N-terminal acylation of peptides was also influenced from the efficient antimicrobial and anti endotoxic activity of Polymyxin B, which is a lipopeptide obtained from natural sources [120]. Lactoferricin is the antimicrobial 
product of invivo cleavage of Lactoferrin and is found to exhibit excellent LPS detoxifying activity, particularly 11 residues from its N-terminal named LF11. Nterminal acylation of LF11 revealed that chain length of 12 acyl chains are optimum for its activity, above which the peptide becomes inactive [121]. When the activity of acylated C12-LF11 was compared with nonacylated LF11, C12-LF11 exhibited enhanced activity and LPS neutralization [121]. Studies with model membranes of DPPG and DPPC lipids revealed that the lipopeptide C12-LF11 strongly penetrate into the phospholipid bilayer regardless of the polar head group. Also a hydrophobic mismatch is found to occur between $\mathrm{C} 12$ chain of peptide with $\mathrm{C} 16$ chain of lipids followed by the insertion of hydrophobic residues of LF11. This results in unfavourable energy interaction [122].

In another study, synthetic 12-mer peptides that have both D and $\mathrm{L}$ enantiomers of Leu/Lys residues were tested for the appropriate cationic charge and acylation of the peptides for appropriate acyl chain length [113]. Negatively stained electron micrographs of the peptides in the presence and absence of LPS revealed that the acylated peptide aggregates to oligomeric micelles in the absence of LPS and induces structural changes in LPS [113]. Aggregation of peptides in LPS layer has been shown to result in impermeability of the peptide into the cells resulting in inactiveness of the peptide [123]. Based on the results of 12-mer peptides, it was postulated that the aggregate formation was formed mostly on the fatty acyl chain since the 12-mer peptides remain in monomeric form because of the presence of mixture of $\mathrm{D}$ and $\mathrm{L}$ forms. The fatty acyl aggregates can easily dissociate upon contact with lipidA. Whereas in the case of aggregation of non acylated peptides, oligomerization is induced in the peptide chain itself, which makes it difficult to traverse the LPS layer [113].

SC-4 is another 12-mer peptide, which shows remarkable activity against Gram negative bacteria but moderate activity towards Gram positive bacteria [124]. Acylation of SC4 peptide showed about 30-fold increase in activity towards Gram positive bacteria including the drug resistant S.aureus strain. The increased hydrophobicity resulting from the acylation is found to overcome the thick peptidoglycan layer in Gram positive bacteria [124]. Studies on acylated analogs of 
Mastoporan X, isolated from wasp venom revealed that an elevated levels of hydrophobicity beyond threshold levels results in loss of membrane selectivity [125].

On the whole, acylation enhances bactericidal activity and LPS neutralization activity only when the chain is of appropriate length, which differs for different AMPs.

\subsubsection{Chimerisation of peptide scaffolds:}

Synergism of antimicrobial peptides can be defined as the interaction of two or more AMPs in combination than as individual peptides itself [126]. This kind of complimentary interaction was first noted in Magainin 2A and PGLa, as both these peptides were present in the same tissue in frog skin [127]. Later on, the concept of synergism were found in other AMPs derived from frog like Temporins [109]. This idea of synergism incited a new concept of forming hybrid or chimera peptides [128]. When Magainin-2 was crosslinked with PGLa with cysteine residue at both terminus resulted in the formation of heterodimers that were more active than the individual parent peptides itself [128].

Conjugation of AMPs of different origin were started to emerge soon. Hybrid peptides composed of Cecropin A from insect moth and Magainin 2 from frog skin exhibited highest antimicrobial activity with no hemolytic activity [129]. This conjugation and its analog peptides also exhibited anti tumor and antiviral properties. Likewise hybrid peptides of N-terminal residues of Cecropin A and Melittin also resulted in enhanced antibacterial peptides with no hemolytic activity at its MIC concentration [129]. Combinatorial chemistry approach of Cecropin A - Melittin hybrid generated BP100, which was active against a variety of phytopathogens [130]. BP100 was active against only Gram negative bacteria and mutation of all of its lysine to arginine residues rendered it to exhibit broad spectrum of activity with less cytotoxicity [131].

In Human beta defensins, HBD-2 and HBD-3, apart from the conserved disulphide bridging, two glycine residues were also found to be present in the same positions. Furthermore, HBD2 was found to be active against E.coli cells at $100 \mathrm{mM} \mathrm{NaCl}$, but inactive against S.aureus. The opposite was found to be true for HBD3[132]. Chimerisation of HBD3 and HBD2 resulted in the identification of two hybrids, C3composed of N-terminal HBD3 until first glycine and rest of HBD2 and C5-composed 
of N-terminal and C-terminal HBD3 with central HBD2. These two hybrids were found to be salt tolerant and found to exhibit broad spectrum of activity even against hospital microbial strains [133].

\subsubsection{Dimerization or cyclization of linear peptides:}

Cyclization is one of the modifications of AMPs that help in protease resistance and mediates the clustering of aromatic residues that aid in enhanced activity [134]. The cyclization is initiated by the addition of cysteine residues at both ends of the linear peptides and then cyclized through disulfide bridges in head to tail fashion. Cyclized melittin exhibited enhanced antimicrobial activity with reduced hemolytic activity, whereas cyclized magainin had reduced antimicrobial activity when compared to linear peptides [135]. The interaction of cyclized peptides with zwitterionic PC membranes was greatly reduced when compared to linear peptides. In fact, the cytolytic activity of cyclized magainins reduced in both bacteria and mammalian cells, because of the reduction in helix content when compared to linear peptides. Whereas in the case of melittin, upon cyclization, all of the cationic residues are confined to small part of the ring and rest of the ring is occupied with hydrophobic residues [135]. Cyclization of insect AMPs, Drosocin and Apidaecin, rendered the peptides inactive against both Gram negative and Gram positive bacteria. The extended central domain of the peptides that are realized as the bioactive center of the peptide was lost during cyclization [136].

\subsubsection{Linearization of dimeric peptides:}

The peptides that bear disulphide bonds fold in aqueous state itself, which upon membrane interaction gets stabilized. Deletion of cysteines in Tachyplesin, a horse shoe crab AMP, retained broad spectrum antibacterial activity but reduced hemolytic activity [137]. But deletion of all cysteines in human neutrophil defensins, HNP-1 abolished antimicrobial activity completely [138]. 


\subsubsection{End tagging of AMPs:}

End tagging of antimicrobial peptides with hydrophobic stretches has been found to be one of the remarkable modifications. Even though increase in overall hydrophobicity can be achieved through various other means, cholesterol excludes such tag insertion. Hence selectivity can be attained with protease and salt resistance activity with end tagging of AMPs [139]. Ultra short AMPs suffer from hydrophobic deficiency even though they can bind to membranes. In such cases, stretches of W/F can compensate for the hydrophobicity needed to kill membranes even in higher salt concentrations [140]. Such a modification was applied to KNK-10 peptide derived from Kininogen. Even if the peptide is truncated to four residues with tryptophan tag, the peptide showed remarkable antibacterial activity with reduced hemolytic activity [140].

\subsubsection{Inclusion of non natural and bulky aminoacid residues:}

Like the inclusion of D-enantiomers, replacement of $\alpha$-aminoacids with $\beta$-aminoacids also provides extensive stability towards proteases and other enzymes [141]. Studies on ultra short lipopeptides like C16-KGGK, C16-KKKK, C16-KAAK peptides retained their antimicrobial activity with the introduction of $\beta$-aminoacids [141]. In the same way, inclusion of bulky aminoacids such as $\beta$-naphthylalanine (Nal) or Biphenylalanine (Bip) to short peptides increases hydrophobic surface of the peptides and thus provides AMPs with salt and serum stability [142].

\subsection{NMR structures aid in the design of novel antiendotoxic AMPs:}

In order to understand the interaction of antimicrobial peptides on membranes and to resolve its mode of action, atomic resolution of AMPs in membrane environment is essential [143]. Out of the several methods in practice, solution state NMR has been considered as well accomplished tool to determine the three dimensional structures of AMPs in membrane models. The NMR structures identifies the position of each aminoacid residue and also the presence of certain structural motifs that can be used for the further improvement of AMPs [144]. 
Liposomes are generally considered as excellent membrane mimics because of homogeneity and lamellar arrangement of lipids. SUVs and LUVs result in quite broad NMR signals because of their large size and slow tumbling rate in NMR time scale [143].
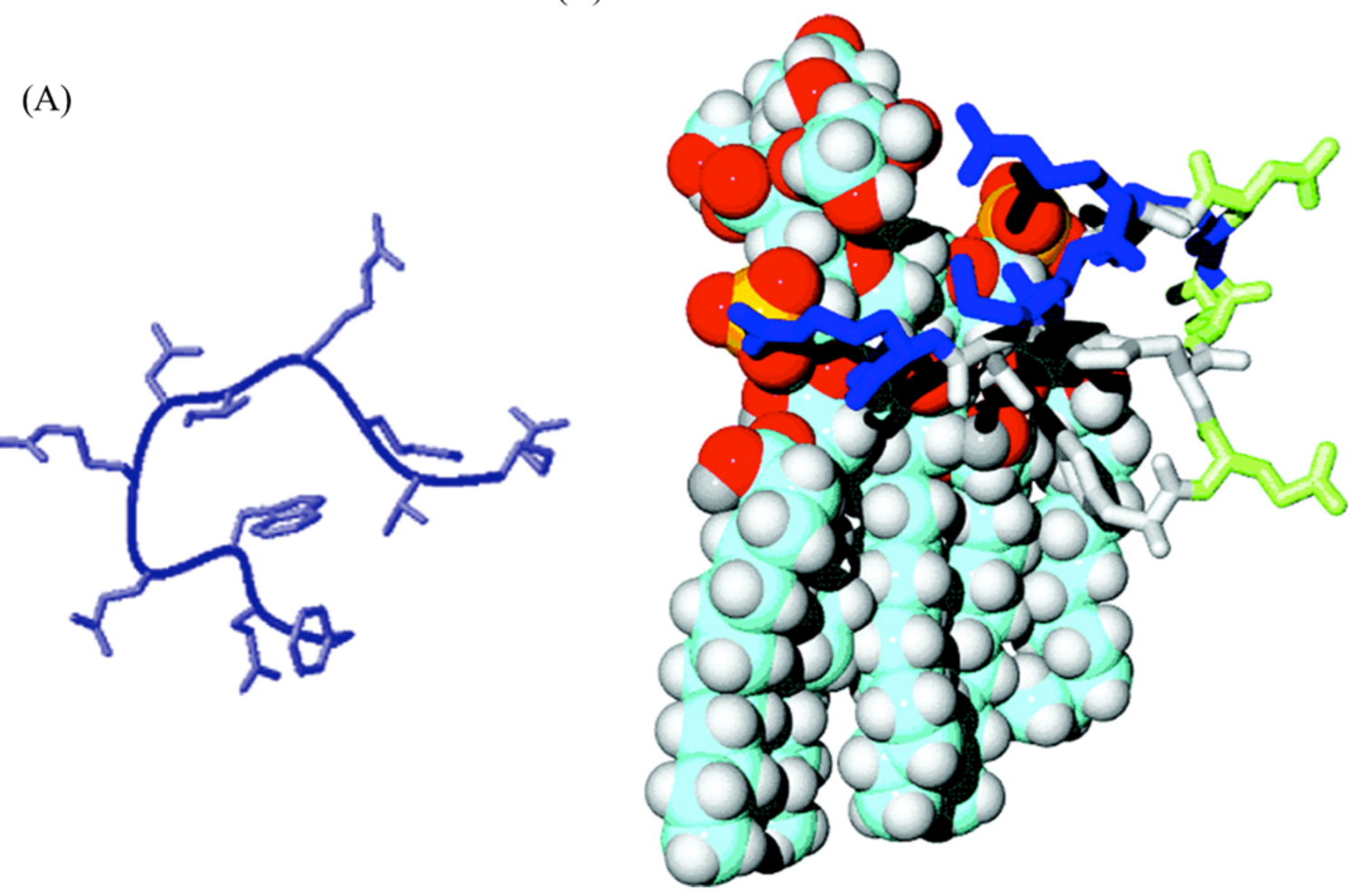

Figure 1.11-1: SAR studies of LF11, a human lactoferrin based peptide: (A) single model of LF11 peptide bound to LPS micelles and (B) Docked model of LF11 in LPS micelle. The color codes of LF11 are blue for cationic residues, green for polar residues and white for hydrophobic residues. Taken from ref [145].

Hence the determination of structures is carried out in micelles, because of its small size and faster tumbling rate that display reasonable NMR spectra for analysis. SDS, anionic micelle that resembles bacterial membranes and DPC, zwitterionic micelle for eukaryotic membranes, generally provides the membrane environment for AMP structure determination. Eventhough LPS also known to form micelles, it forms remarkably huge aggregates that produce broad NMR signals. Transfer-NOESY experiments emerged to be highly successful in such cases. Typically, low 
concentration of LPS micelles is titrated into peptide solutions of higher concentration. If the peptides are binding to LPS with moderate affinity, then the dissociation will be faster giving rise to tr-NOE peaks. The intensity of tr-NOE peaks is enhanced by the difference between rotational correlation time between free peptide and LPS-peptide complex. Alternatively, if the peptides are binding tightly, the dissociation will be much slower in NMR time scale that precludes the observation of tr-NOEs [62].

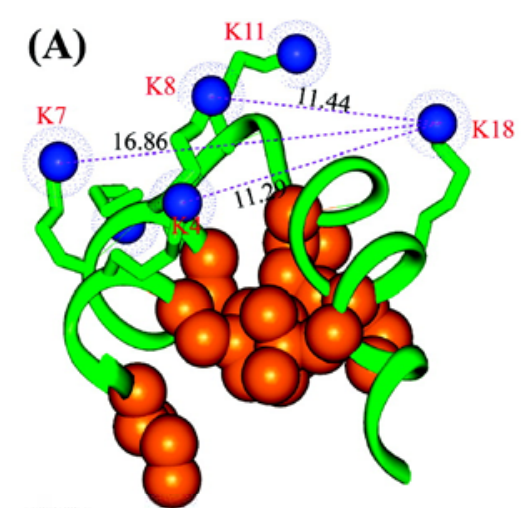

(B)

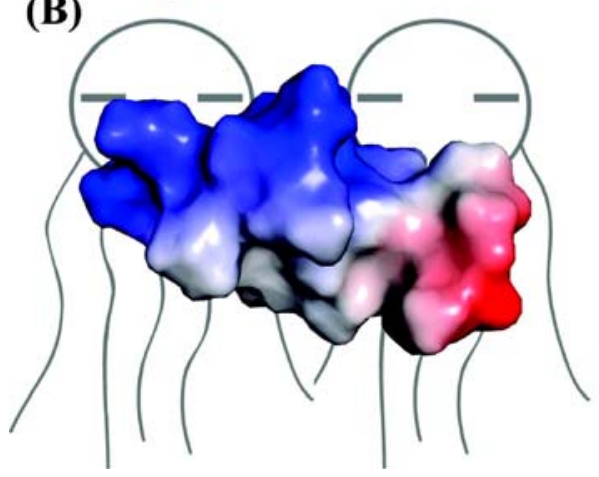

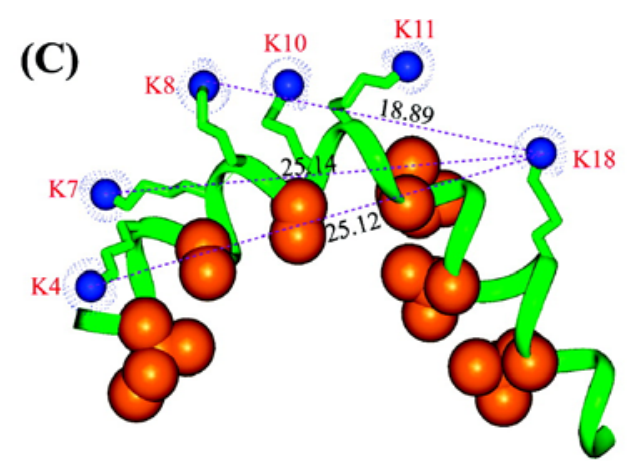

(D)

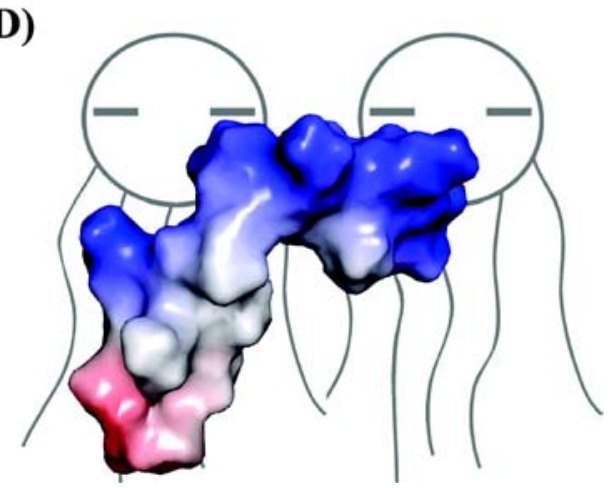

Figure 1.11-2: SAR studies of MSI594, a hybrid peptide of MSI78 and Meltitin and its mutant MSI594F5A: (A) Model of MSI594 bound to LPS micelles with space filling representation of residue $F 5$ in helix 1 with residues in helix 2. (B) Mode of interaction of MSI594 with LPS micelles. (C) Model of MSI594F5A bound to LPS micelles. Distance between nitrogen atoms of side chains of lysine is also mentioned. (D) Mode of interaction of MSI594F5A with LPS micelles. Taken from ref [146].

Structure-Activity relationship studies (SAR) of antimicrobial peptides reveal the specific fold of the peptide upon membrane interaction and involvement of individual 
residues in such interactions [144]. Atomic resolution structure of LF11, a peptide identified from human lactoferrin, in complex with LPS revealed a unique T-fold (Figure 1.11-1, A). The hydrophobic core formed by F1, W3 and I7 occupies the stalk of T-shape and two hydrophilic clusters of Q4R5N6 and R8K9R11 occupy the two sides along the horizontal axis of T-fold (Figure 1.11-1,A) [145]. Docking studies of LF11 structure to LPS disclosed that two arginine residues R1 and R5 interact with phosphoryl group of lipidA and guanidino group in R8 interacting with carboxylate group of KDO (Figure 1.11-1, B).

The three dimensional structure of LF11 in complex with LPS was also found to resemble the LPS interacting interface of FhuA, an outer membrane protein of E.coli. Tryptophan residue, W3 in LF11 is found to be critical in interacting with LPS and also with other hydrophobic residues of the peptide [145]. Hence biophysical and activity characterization of peptides disclose only the activity of the peptides and it can be directly correlated with the atomic resolution structure derived from NMR constraints.

SAR studies of MSI594 and its mutant peptide MSI594F5A serves as another striking example that acknowledges its importance. MSI 594 is a hybrid peptide of MSI78 - an analogue of Magainin and Melittin. Pexiganan or MSI78 exhibit broad spectrum of antibacterial activity and also entered clinical trials for the treatment of diabetic ulcers [147]. The highly cationic face of N-terminus of MSI78 was found to get along with hydrophobic face of N-terminus of Melittin. Hence MSI594 was found to exhibit broad spectrum of antimicrobial activity than MSI78 and reduced hemolytic activity than Melittin [26]. Inorder to reveal the structural fold of this peptide, NMR studies were carried out in LPS micelles. Three dimensional structure of MSI594 in complex with LPS revealed a unique helical hairpin fold with Phe5 in helix 1 interacting with Ileu13, Val16 and Leu17 in helix 2 (Figure 1.11-2, A). The amphipathicity was induced by the exposure of all lysine residues on one side and interaction of hydrophobic residues on both helices on other side (Figure 1.11-2, B) [26]. Thus it was revealed that this amphipathic structure of unique helical hairpin fold act as driving force behind the enhance membrane permeability and activity. Eventhough MSI594 was found to adopt helical structure with a bend in DPC micelles, the packing 
interactions between helices in helical hairpin fold are found to confine to LPS micelles.

In order to ascertain the importance of Phe5 residue in MSI 594, it was mutated to alanine to form MSI594F5A. The antimicrobial activity against Gram negative bacteria was found to be reduced by several folds when compared to MSI594. Also the outer membrane permeability was affected as the affinity for binding to LPS decreased [146]. But the global conformation by CD revealed alpha helical structure for MSI594F5A. Hence the exact reason behind the lowered activity was revealed only by three dimensional structure determination in LPS. The peptide MSI594F5A adopted "V" shaped helical conformation. But the close contact of residues in two helices as observed in MSI594 was not present in MSI594F5A because of the mutation of critical residue F5 (Figure 1.11-2, C). Even though the side chain of lysine residues are exposed on one side of the helix, the distance between them was greater when compared to MSI 594 (Figure 1.11-2, C). Since because the cationic distribution has scattered, the peptide cannot efficiently overcome the outer membrane permeability barrier (Figure 1.11-2, D) [146].

\subsection{Design of $\beta$-boomerang peptides:}

Atomic resolution co-crystal structure of Fhu A, outer membrane protein of E.coli in complex with LPS displayed a clear understanding of interfacial residues of the

protein that make close contact with LPS (Figure 1.12-1, A). It revealed that the protein adopted $\beta$-barrel in $\mathrm{C}$-terminal and cork domain in $\mathrm{N}$-terminal [148]. The residues in C-terminal $\beta$-barrel were found to make close contact with LPS. The basic residues K351, R384 K431 and K441 were found to interact with diphosphoryl groups of lipidA. Aromatic and hydrophobic residues like F302, F355 and F380 were found to interact with upper part of lipidA acyl chain and L300, Y284 and F235 were found to interact with lower acyl chains (Figure 1.12-1, A). The four basic residues that bind to phosphoryl group were found to be common among other LPS binding proteins such as BPI and LALF [148]. 
(A)

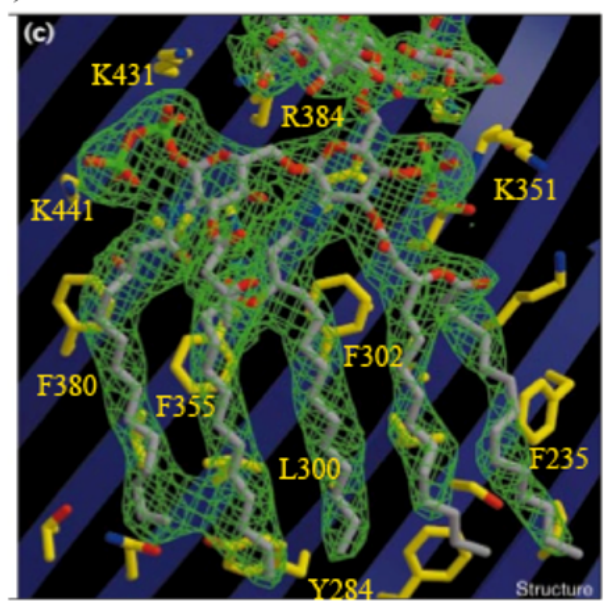

(B)

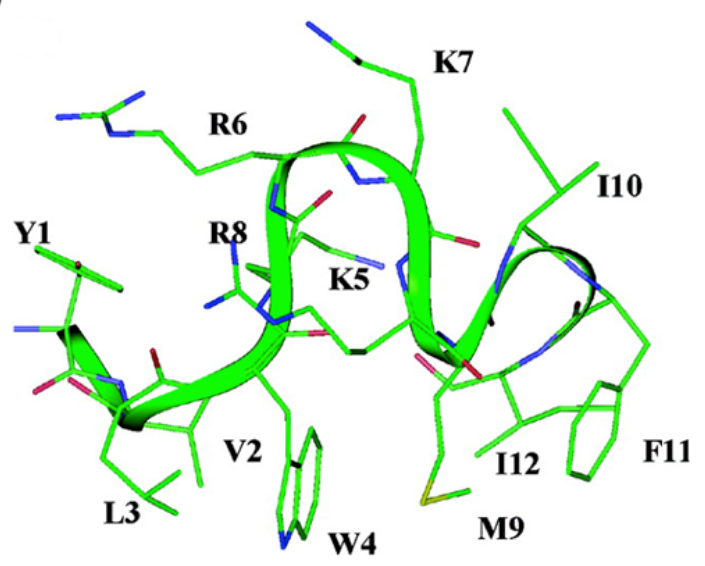

(C)

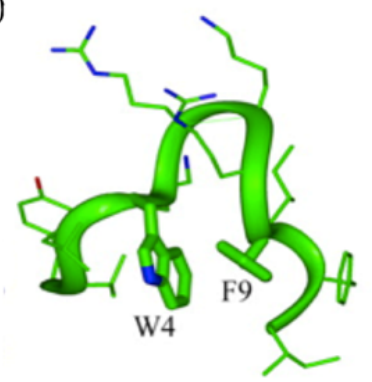

(D)

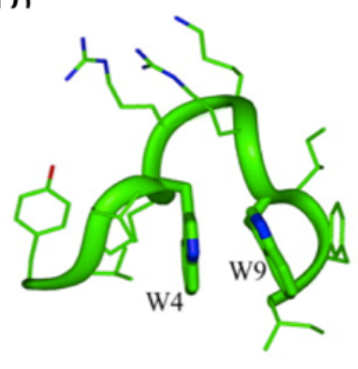

(E)

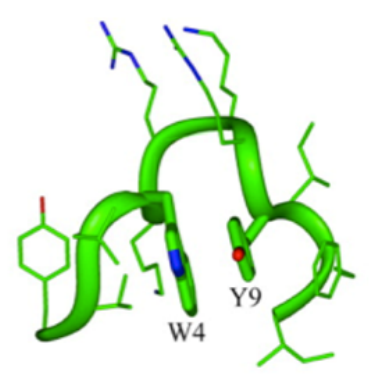

Figure 1.12-1: Design of $\beta$-boomerang peptides: Top panel: (A) Electron density map of interfacial residues of FhuA, an outer membrane protein (marked with yellow color) with LPS (displayed as mesh). Taken from ref [148]. (B) Single model representation of YI12 peptide, parent peptide of $\beta$-boomerang. Taken from ref [149]. Bottom panel: Single model representations of second generation of $\beta$-boomerang peptides, (C) YI12WF, (D) YI12WW and (E) YI12WY. Taken from ref [150].

An antimicrobial peptide (YI12 - YVLWKRKRMIFI) was designed based on this LPS binding residues of FhuA protein (Figure 1.12-1, B). Atomic resolution structure of this peptide in LPS micelles revealed that the peptide folds into a unique shape with all of the four cationic residues on one side with hydrophobic residues on the other side [149]. Interestingly, close contact between W4 and M9 residue was observed imparting the peptide, a boomerang fold. This peptide was found to be active against bacteria with moderate LPS neutralization potency [149]. 
In the second generation, M9 residue was replaced with aromatic aminoacids, Phe (YI12WF), Trp (YI12WW) and Tyr (YI12WY) in order to enhance packing of the hydrophobic residues for improved LPS detoxification activities (Figure 1.12-1, Bottom panel). Three-dimensional structure determination of these peptides in LPS micelles revealed that all of the peptides conserved the unique boomerang fold with enhanced aromatic packing between position 4 and 9 [150]. Activity studies and biophysical characterization of all these peptides disclosed that these peptides can detoxify LPS at concentrations as low as $10 \mathrm{nM}$ [150].

The second generation peptide studies also identified a structured LPS binding motif composed of W-K-R-K-R-F, when the aromatic lock with cationic residues of YI12WF peptide was truncated with glycine (GG8WF) [150]. This peptide was found to interact with LPS probably because of the presence of cationic residues but the hydrophobic interactions required to kill bacteria were not met because of the truncation. Structure of GG8WF peptide in LPS micelles revealed that the peptide folds into characteristic boomerang shape with aromatic look between W2 and F7. Hence this motif was recognized as structured LPS binding motif [150].

In this work, strategies like N-terminal acylation, chimerization, dimerization through cysteine disulfide bridges of $\beta$-boomerang peptides were tested for the successful design of antimicrobial antiendotoxic peptides.

\subsection{Objectives of the current work:}

Lipopolysaccharides, LPS, an integral glycolipid of outer membrane of Gram negative bacteria serves to trap hydrophobic agents such as antimicrobial peptides, modification of which has been shown to involve in antibiotic resistance [86]. Structured LPS binding motif identified from a library of $\beta$-boomerang YI12 peptides [150] is utilized here for further refinement towards development of antimicrobial antiendotoxic agents 


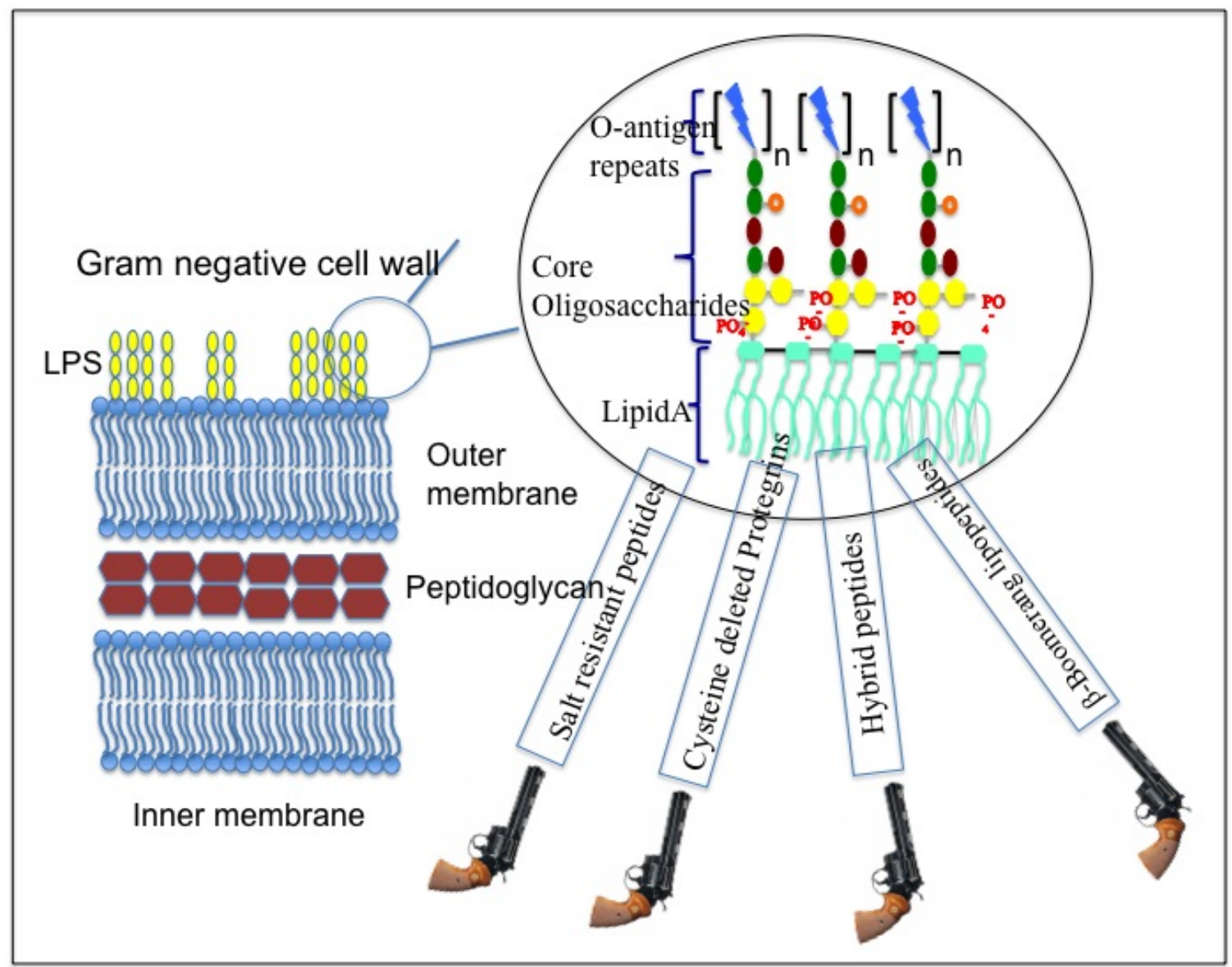

Figure 1.13-1: Schematic representation of the objective of current work: Lipopolysaccharides, LPS that are present in the outer layer of outer membrane of Gram-negative bacteria is targeted towards the design of antimicrobial and antiendotoxic agents.

. Four different rationales were devised in the current work that involves

* N-terminal acylation and C-terminal dimerization of $\beta$-boomerang peptide

* Hybrid peptides that involves conjugation of LPS binding motif with LPSinactive peptides

* Cysteine deleted Protegrin-1 analogs and

* Salt resistant alpha helical peptides. 
CHAPTER 2

\section{MATERIALS AND METHODS}




\section{MATERIALS AND METHODS}

\subsection{Materials:}

Chemicals like DMSO, $\mathrm{NaCl}, \mathrm{KCl}$, Tris buffer, EDTA, Glucose, Acrylamide, HEPES buffer, $\mathrm{Na}_{2} \mathrm{HPO}_{4}, \mathrm{NaHPO}_{4}$ were ordered from Sigma-Aldrich (St.Louis, MO). LPS from E.coli 0111:B4, FITC-LPS from E.coli 055:B5, SDS and fluorescent dyes like NPN, Calcein were also purchased from Sigma-Aldrich (St.Louis, MO). SYTOX green was purchased from Invitrogen and LAL kit was purchased from Lonza. Lipids like POPC, POPG, DPC and the extruder that was used for preparing liposomes were purchased from Avanti Polar Lipids (Alabaster, AL). Di-S-C 3 dye was procured from Fluka Biochemika. NMR reagents like DSS, $\mathrm{D}_{2} \mathrm{O}$ and DPC- $\mathrm{d}_{38}$ were purchased from Cambridge Isotope Laboratories, Inc (Massachusetts, USA).

\subsection{Methods:}

\subsubsection{Purification of crude peptides by HPLC:}

The crude peptides from GL Biochem, Shangai, China were dissolved in $90 \%$ water and $10 \%$ acetonitrile with $0.1 \%$ TFA. The dissolved samples were filtered through $0.45 \mu \mathrm{m}$ filters to get rid of fine undissolved particles. The filtered samples were then injected into semi-preparative cell of reverse phase HPLC (Waters) that is connected to a $\mathrm{C} 18$ column (300 $\AA$ pore size, $5 \mu \mathrm{m}$ particle size). A linear gradient of $90 \%$ water, $10 \%$ acetonitrile, both with $0.1 \%$ TFA and a flow rate of $2 \mathrm{ml} / \mathrm{min}$ was used to purify the injected peptide. The various peptide components according to its hydrophobicity get separated in the column and passes through the UV monitor. The purification process is monitored through the chromatograms at $220 \mathrm{~nm}$ and $278 \mathrm{~nm}$ for peptide bonds and tryptophan absorbance (if at all present) respectively. The major peaks eluting with high absorbance were collected, lyophilized and used for further experiments. 


\subsubsection{Oxidation of third generation $\beta$-boomerang hybrids:}

Peptides that contain cysteine were oxidized with $20 \%$ DMSO as explained in [151]. The crude peptides were dissolved in $20 \%$ DMSO and the $\mathrm{pH}$ was raised to 9.0 to enhance the formation of disulfide bonds. This mixture was incubated for $18 \mathrm{hrs}$ and then stopped by adding water acetonitrile mixture. The samples were then lyophilized to remove DMSO. The lyophilized samples were then purified by semi preparative HPLC as mentioned above.

\subsubsection{Activity assays:}

\subsubsection{Minimum inhibitory concentration determination (MIC):}

Antimicrobial activities of the peptides were determined using broth dilution method. The bacterial strains except E.coli and B.subtilis used were procured from American type culture collection, (Rockville, MD) and their ATCC numbers are listed below.

Table 2.2-1: Bacterial strains and respective ATCC numbers.

\begin{tabular}{|l|l|}
\hline Bacteria & ATCC numbers \\
\hline Escherichia coli & Lab strain \\
\hline Pseudomonas aeruginosa & ATCC 27853 \\
\hline Klebsiella pneumoniae & ATCC 13883 \\
\hline Salmonella enterica & ATCC 14028 \\
\hline Bacillus subtilis & Lab strain \\
\hline Staphylococcus aureus & ATCC 25923 \\
\hline Streptococcus pyogenes & ATCC 19615 \\
\hline Enterococcus faecalis & ATCC 29212 \\
\hline
\end{tabular}

Frozen glycerol stocks of these strains were thawed and streaked onto LB agar plates to accomplish single colonies. One of the single colonies was then grown overnight in LB broth at $37^{\circ} \mathrm{C}$. Next day, mid-log phase cultures were obtained from overnight grown bacteria cells in $\mathrm{MH}$ broth. They were then diluted in $\mathrm{MH}$ broth to an $\mathrm{OD}_{600 \mathrm{~nm}}$ of 0.01. Minimum inhibitory concentration determination assay was initiated by 
preparing around $50 \mu \mathrm{L}$ of two fold dilution of the peptides in concentration ranging from $0.2 \mu \mathrm{M}$ to $200 \mu \mathrm{M}$ in water in 96-well micro titer plates. Equal volumes of the above-diluted bacteria were added to these plates and this mixture was then incubated for $16 \mathrm{hrs}$ at $37^{\circ} \mathrm{C}$. Wells with only water in the place of peptides were used as negative control. MIC was determined by measuring the OD of the plates and the concentration at which there is complete inhibition of bacterial growth was recorded as the MIC of the peptide. For the determination of MIC values in the presence of salt, mid-logarithmic phase of bacterial cells were obtained in $\mathrm{MH}$ broth with appropriate concentration of $\mathrm{NaCl}$ and further dilutions were carried out in same broth.

To differentiate between bacteriostatic and bactericidal effect of the peptides, the wells that show constant decrease in OD was plated in $\mathrm{MH}$ agar plates and incubated overnight. The plates were then examined for bacterial growth and compared with MIC results.

\subsubsection{Hemolytic assay:}

The ability of the peptides to disrupt red blood cells was assayed using the protocol as described by Shai et.al [152]. Briefly, the RBCs with EDTA were centrifuged at $800 \mathrm{~g}$ for $10 \mathrm{~min}$ to remove the buffy plasma coat layer. Then the resulting RBCs were resuspended in PBS and washed three times. Around $50 \mu \mathrm{L}$ of suspended RBCs were added to equal volume of two-fold dilution of the peptides in 96-well micro titer plates and incubated for one hour. The final erythrocyte concentration is $4 \%(\mathrm{~V} / \mathrm{V})$. After one hour, the mixture was centrifuged and the release of hemoglobin in the supernatant was determined spectrophotometrically at $\mathrm{OD}_{540 \mathrm{~nm}}$. Buffer in the place of peptides served as negative control and $1 \%$ triton-X served as positive control. The percentage of hemolysis was calculated using the following formula:

$\%$ of hemolysis $=\frac{\text { ODpeptide }- \text { ODblank }}{\text { ODtritonX }- \text { ODblank }} \times 100$

\subsubsection{Limulus amoebocyte lysate (LAL) assay:}

The strength of the peptides to neutralize LPS was assayed using commercially available LAL chromogenic kit (QCL 100 Cambrex). The protocol explained in the 
manufacturer's instructions was strictly adhered. The endotoxic principle, LPS in Gram negative bacteria activates a proenzyme in Limulus amoebocyte lysate (LAL). This activated enzyme in turn catalytically splits colored product pNA from the colorless substrate Ac-Ile-Glu-Ala-Arg-pNA and is detected spectrophotometrically at $\mathrm{OD}_{410 \mathrm{~nm}}$. The peptides were dissolved in the pyrogen free water supplied with the kit and $\mathrm{pH}$ was adjusted to 7.0 with $1 \mathrm{~N} \mathrm{HCl}$ or $1 \mathrm{~N} \mathrm{NaOH}$ (which is also prepared in pyrogen free water). The increasing concentrations of the peptides were incubated with 1.0 EU (Endotoxin units) in a total volume of $50 \mu \mathrm{L}$ for 30 minutes at $37^{\circ} \mathrm{C}$. About $50 \mu \mathrm{L}$ of LAL reagent was added to peptide-EU complex and further incubated for 10 minutes followed by addition of $100 \mu \mathrm{L}$ substrate. After incubation of six minutes for the reaction, the release of colored product was recorded at $\mathrm{OD}_{410 \mathrm{~nm}}$. Water in the place of peptides served as negative control (blank) that is considered as $0 \%$ inhibition and percentage of LPS neutralization was calculated by

$\%$ of LPS neutralization $=\frac{O D(\text { blank })-O D(\text { peptide })}{O D(\text { blank })} \times 100$

\subsubsection{Zeta potential measurements:}

The zeta potential measurements were carried out on a Zeta sizer Nano ZS (Malvern Instruments, Worcestershire, UK) equipped with a $633 \mathrm{~nm}$ He lasers. For bacterial cell measurements, bacteria were grown to mid log phase in LB media and diluted to an $\mathrm{OD}_{600 \mathrm{~nm}}$ of 0.2 . Zeta potential of around $750 \mu \mathrm{L}$ of bacterial cells were first measured followed by the addition of increasing concentration of peptides. The measurements were made in disposable zeta cells with gold electrodes and the samples were allowed to equilibrate for $120 \mathrm{sec}$ at $25^{\circ} \mathrm{C}$. A total of 5 measurements of 100 runs each were carried out for all dilutions.

\subsubsection{Biophysical characterization}

\subsubsection{Intrinsic tryptophan fluorescence:}

All of the fluorescence measurements were carried out with Cary Eclipse fluorescence spectrophotometer (Varian Inc) equipped with dual monochromators. Measurements were made using a $0.1 \mathrm{~cm}$ path length quartz cuvette. 
The intrinsic tryptophan fluorescence spectra of the peptides were measured in $10 \mathrm{mM}$ phosphate buffer, $\mathrm{pH} 7.0$ unless otherwise specified. The peptides were excited at 280 $\mathrm{nm}$ and emission was collected from 300-400 nm with slit width of $5 \mathrm{~nm}$. The experiment was initiated by measuring fluorescence of about $5 \mu \mathrm{M}$ of the peptides followed by the addition of increasing concentration of LPS or DPC or SDS micelles.

\subsubsection{Acrylamide quenching:}

The surface accessibility of tryptophan residues in different micellar environments was studied using acrylamide quenching where acrylamide is a neutral quencher. The rate of decrease in fluorescence intensity after addition of increasing concentration of acrylamide was measured using Stern-Volmer $\left(\mathrm{K}_{\mathrm{sv}}\right)$ constant with the equation $\frac{F 0}{F}=1+K s v[Q]$

F0 and F are the intensities before and after addition of quencher and [Q] is the molar quencher concentration. In order to compare the solvent accessibility of tryptophan to quencher, experiments were performed for both free peptide and Peptide/micelle complex.

\subsubsection{Outer membrane permeabilization assay:}

Outer membranes in the bacteria act as permeability barrier for most of the hydrophobic antibacterial compounds designed. Hence the ability of the designed peptides to permeabilize through the outer membrane was examined using $1-\mathrm{N}$ Phenylnaphthyl-amine (NPN) dye. E.coli cells were grown to mid logarithmic phase and diluted to an $\mathrm{OD}_{600 \mathrm{~nm}}$ of 0.5 in $10 \mathrm{mM}$ phosphate buffer, $\mathrm{pH}$ 7.0. The excitation was set at $350 \mathrm{~nm}$ and emission at $390-450 \mathrm{~nm}$. NPN was added to the diluted cells at the concentration of $10 \mu \mathrm{M}$ and a basal fluorescence was recorded followed by the addition of increasing concentration of peptides.

\subsubsection{Inner membrane permeability assay:}

E.coli cells were grown to logarithmic phase in LB media as mentioned above and diluted to an $\mathrm{OD}_{600} \mathrm{~nm}$ of $0.5 \mathrm{in} 10 \mathrm{mM}$ phosphate buffer, $\mathrm{pH}$ 7.0. Around $1 \mu \mathrm{M}$ of 
SYTOX green dye was added to the cells and incubated in dark for 15 min with shaking. A basal fluorescence was recorded with excitation at $485 \mathrm{~nm}$ and emission at $490-550 \mathrm{~nm}$. The ability of the peptides to disrupt inner membrane was recorded by adding increasing concentration of the peptides to the above mixture.

\subsubsection{Calcein leakage assay:}

Membrane permeabilizing ability of the peptides invitro was checked using the release of calcein from liposomes. Liposomes have been widely used to mimic the bacterial and mammalian membranes closely. Liposomes of POPG, POPC, POPC: POPG (3:1) and POPC: LPS (80:20) were prepared by extrusion technique. The dry weight of the appropriate concentration of the lipids was measured in glass vials. Chloroform: methanol (3:1) was added to the vials to briefly dissolve the lipids and the solvent was evaporated under a stream of nitrogen gas to form a thin lipid film on the sides of the vial. It was freeze dried for $2 \mathrm{hrs}$. to completely remove the solvent. This lipid film was then dissolved in $10 \mathrm{mM}$ Tris buffer that contains $60 \mathrm{mM}$ calcein (self quenching concentration). The vial was vortexed vigorously to form multilamellar (MLV) liposomes. The mixture was frozen and thawed alternatively at least for 10 times. The MLVs thus formed was made unilamellar by extruding the lipid through two stacked $100 \mathrm{~nm}$ membranes. The lamellarity of the liposomes thus made was confirmed with dynamic light scattering measurements.

Unbound calcein was removed from calcein-trapped liposomes using PD-10 desalting columns. The columns were first washed and equilibrated with $10 \mathrm{mM}$ tris buffer followed by the addition of extruded liposomes. As it passes through the column the calcein entrapped liposomes elute first followed by the free calcein. The volume of the buffer used to elute calcein-entrapped liposomes was recorded to calculate the dilution factor.

The leakage of calcein from disrupted liposomes can then be measured fluorometrically by exciting the vesicles at $490 \mathrm{~nm}$ and emission at $500-550 \mathrm{~nm}$. Increasing concentration of the peptides was added to $50 \mu \mathrm{M}$ liposomes and increase in fluorescence intensity was noted. 


\subsubsection{Membrane depolarization measurements:}

The assay to measure membrane depolarization ability was performed in intact E.coli cells and its spheroplasts.

For intact cells, the bacteria was grown to mid log phase, centrifuged and suspended in $5 \mathrm{mM}$ HEPES and $20 \mathrm{mM}$ glucose at $\mathrm{pH}$ 7.4. After a brief wash, the cell pellets were suspended in the same buffer with $100 \mathrm{mM} \mathrm{KCl}$ to an $\mathrm{OD}_{600 \mathrm{~nm}}$ of 0.05 . The spheroplasts were prepared by suspending the log phase grown bacterial pellets in 10 $\mathrm{mM}$ Tris, $25 \%$ Sucrose $\mathrm{pH}$ 7.4. The pellets were washed twice and resuspended in the same buffer, but this time containing $1 \mathrm{mM}$ EDTA. The cells were incubated for 15 min with shaking and centrifuged. The pellets were immediately dissolved in ice-cold water and further incubated for $10 \mathrm{~min}$ at $4^{\circ} \mathrm{C}$. The cells devoid of outer membrane i.e.

Spheroplasts were collected by centrifugation and the pellets were suspended to an $\mathrm{OD}_{600 \mathrm{~nm}}$ of 0.05 in same buffer $(5 \mathrm{mM}$ HEPES, $20 \mathrm{mM}$ glucose, $100 \mathrm{mM} \mathrm{KCl}$ ) as intact cells.

The depolarization detecting dye $\operatorname{DiS}^{-C_{3}-5}$ (3,3`-diethylthiodicarbocyanine iodide) dye was added to either intact cells or spheroplasts and basal fluorescence was recorded by exciting the dye at $622 \mathrm{~nm}$ and emission collected at 630-700 $\mathrm{nm}$. This was incubated for about 45-60 minutes until the decrease in fluorescence in stable i.e. the dye partitions into the membrane. This was then followed by the addition of increasing concentration of different peptides and the depolarizing ability was recorded as the measurement of increase in fluorescence intensity.

\subsubsection{FITC-LPS dequenching experiment:}

Lipopolysaccharides are one of the most important target molecules in the development of antibacterial compounds. LPS molecules form micellar aggregates when dissolved in water. The competency of the peptides to dissociate the LPS micelles were quantified using FITC conjugated LPS. In this method, blank fluorescence of $500 \mathrm{nM}$ of FITC-LPS was recorded followed by the addition of increasing concentration of the peptides in $10 \mathrm{mM}$ phosphate buffer, $\mathrm{pH}$ 7.0. The excitation was set at $480 \mathrm{~nm}$ and emission at 500-550 $\mathrm{nm}$. 


\subsubsection{Dynamic light scattering:}

Lipopolysaccharides, when suspended in aqueous media form aggregates or micelles, which is considered to be its active form. The LPS molecules are held together by $\mathrm{Mg}^{2+}$ divalent cations through electrostatic attractions. The competing ability of the peptides against divalent cations was checked with dynamic light scattering. LPS forms micelles of bigger size, which with the addition of increasing concentration of peptides, dissociates to smaller sizes. Measurements were made for $0.5 \mu \mathrm{M}$ LPS and then with LPS: Peptide ratios of 1: 1 and 1: 2 . The scattering was measured with Dynamic Light Scattering software provided with the instrument (Brookhaven Instruments Corp., Holtsville, NY) and the scattering data was analyzed with CONTIN method.

\subsubsection{Isothermal titration calorimetry:}

The thermodynamic principles of the binding of the peptides with LPS micelles were determined with Isothermal titration calorimetry using VP-ITC Micro calorimeter (Microcal Inc, Northampton, MA). Peptides and LPS was dissolved in $10 \mathrm{mM}$ phosphate buffer, pH 7.0 and filtered. LPS at a concentration of $10 \mu \mathrm{M}$ was loaded into the sample cell and the reference cell was filled with the buffer. The syringe was filled with $1 \mathrm{mM}$ peptide stock. Typically 25 or 10 (in some cases) injections of $3.5 \mu \mathrm{L}$

of the peptides were made into the sample cell at $25^{\circ} \mathrm{C}$. The sample cell was stirring continuously at $300 \mathrm{rpm}$. Raw data was collected and fitted using single site binding model in Microcal Origin 5.0 software.

Association constant $\left(\mathrm{K}_{\mathrm{a}}\right)$ and enthalpy change $(\Delta \mathrm{H})$ were directly obtained from the software used. Dissociation constant was calculated using formula $K_{d}=1 / K_{a} . \Delta G$ and $\mathrm{T} \Delta \mathrm{S}$ were calculated using the fundamental equations of thermodynamics, $\Delta \mathrm{G}=$ $R T \ln K_{a}$ and $T \Delta S=(\Delta H-\Delta G)$ respectively. 


\subsubsection{Structural characterization:}

\subsubsection{Circular Dichroism spectroscopy:}

Conformational change of the peptides upon binding to membranes was detected using CD spectroscopy. Data were collected using a Chirascan CD spectrometer (Applied Photophysics Ltd., UK) and Jasco-815 spectrometer. The peptide and peptide/micelle complex were scanned from 190 to $240 \mathrm{~nm}$ wavelengths in a $0.01 \mathrm{~cm}$

path length cuvette for an average of 3 scans. Baseline scans were acquired using 10 $\mathrm{mM}$ phosphate buffer, $\mathrm{pH}$ 7.0. LPS, DPC and SDS micelles baselines were also acquired with same settings. About $20-50 \mu \mathrm{M}$ of peptides was used with $10-30 \mu \mathrm{M}$ of LPS, 15-20 mM DPC and $20 \mathrm{mM}$ SDS to obtain secondary structure of the peptide in different micellar environments. The appropriate baselines were used to subtract the data. For molar ellipticity conversions, Pro-data viewer was used for Chirascan measurements and for Jasco measurements, $[\theta]=\theta / 10 x c x l$ formula was used where c is the concentration of peptide used and 1 is the pathlength of the cuvette.

\subsubsection{NMR spectroscopy:}

All of the NMR spectra were recorded on a Bruker DRX 600 spectrometer, equipped with a cryo-probe and pulse field gradients. Data acquisition and processing were performed with the Topspin software (Bruker) running on Linux workstation. Two dimensional TOCSY (total correlation spectroscopy) and NOESY (nuclear overhauser effect spectroscopy) spectra of peptides in free solution were acquired in aqueous solution containing $10 \% \mathrm{D} 2 \mathrm{O}$ at $\mathrm{pH} 4.5$ with $0.5 \mathrm{mM}$ peptide concentration. The mixing times were $80 \mathrm{~ms}$ and $250 \mathrm{~ms}$ respectively. DSS (2,2-dimethyl-2-silapentane 5-sulfonate sodium salt) was used as an internal standard. The experiments were performed at $298 \mathrm{~K}$ and if there are spectral overlaps, the spectra were acquired at 278 K. NOESY experiments were performed with 456 increments in $t_{1}$ and $2 \mathrm{~K}$ data points in $t_{2}$ using the WATERGATE procedure for water signal suppression. The spectral width was typically $12 \mathrm{ppm}$ in both dimensions. After zero filling along the $\mathrm{t}_{1}$ dimension, $4 \mathrm{~K}\left(\mathrm{t}_{2}\right) \times 1 \mathrm{~K}\left(\mathrm{t}_{1}\right)$ data matrices were obtained. NMR data processing and 
analysis were carried out using Topspin (Bruker) and Sparky (T.D.Goddard and D.G.Kneller, University of California, San Fransisco, CA) programs respectively.

For trNOESY experiments, $0.5 \mathrm{mM}$ peptide solutions were titrated with different concentrations of LPS from $5 \mu \mathrm{M}$ to $50 \mu \mathrm{M}$. The two dimensional tr-NOESY NMR experiments were acquired with the same parameters as NOESY experiment except with the mixing time of $150 \mathrm{~ms}$.

For NMR experiments of peptides in DPC micelles, $0.5 \mathrm{mM}$ peptide was dissolved in $125 \mathrm{mM}$ DPC- $\mathrm{d}_{38}$. Two dimensional TOCSY and NOESY spectra were obtained at $310 \mathrm{~K}$.

${ }^{13} \mathrm{C}-\mathrm{HSQC}$ (natural abundance) of peptide/micelle complex was also acquired for peptides (wherever applicable) at same temperature and other parameters to derive chemical shift deviations.

\subsubsection{NMR derived structure calculations:}

NMR structures were calculated using CYANA program. Sequential walk of different spin systems were achieved in SPARKY by overlaying two dimensional TOCSY and NOESY spectra of the peptides either free or of peptide/micelle complexes. Further medium range and long range NOEs were assigned and on the basis of cross peak intensities, NOEs were categorized to strong, medium and weak. They were then translated to upper bound distance limits to 2.5,3.5 and 5.0 $\AA$ respectively. To restrict the conformational search, the $\varnothing$ dihedral angles were set to $-30^{0}$ to $-120^{\circ}$ for all residues except for Gly and Pro. Dihedral angles from TALOS were also used wherever ${ }^{13} \mathrm{C}$-HSQC data were obtained. Several rounds of structure calculations were carried out and depending on NOE violations, the distance constraints were increased or decreased inorder to obtain energy minimized structures. Out of 100 structures calculated, 20 lowest energy structures were kept for analyses. PROCHECK was used to analyse Ramachandran plot for the validation of structures calculated. 


\section{CHAPTER 3}

\section{RESULTS AND DISCUSSION}




\section{RESULTS AND DISCUSSION}

\subsection{DIMERIZED $\beta$-BOOMERANG LIPOPEPTIDES}

\subsubsection{Peptide design:}

In an arena for the search of effective antimicrobial compounds to overcome the development of antibiotic resistant strains, several modifications have been performed on the ribosomally or non ribosomally synthesized peptides. Addition of bulky hydrophobic residues or non-natural aminoacids, end tagging of peptides, inclusion of diastereomers, additions of long fatty acid chains of different lengths etc. are some of the modifications known to date. Addition of fatty acyl chain in antimicrobial peptides had been practiced ever since the discovery of Polymyxin B, which is an acylated AMP. Deacylation of PMB leads to loss of antibacterial activity [153] and increase in the level of toxicity to mammalian cells also. Increased hydrophobicity from fatty acyl chains intertwined with the hydrophobicity of the peptide itself is considered to be an added advantage in the addition of acyl chains to AMPs [154]. Unlike the mutation of residues to d-diastereomers, addition of fatty acid chains of different length does not seem to interfere with the secondary structure of peptides [152].

Among several classes of antimicrobial peptides, $\beta$-hairpins stabilized by disulfide bridges occupy a prominent position. Defensins, Protegrins, Polyphemusins and Tachyplesins are some examples of AMPs that fall under this category. In all of these peptides, the disulfide bridge is found to play an important role in antibacterial activity and cell selectivity. Moreover the presence of disulfide bridge stabilize folded conformations of AMPs in aqueous solutions which upon membrane interactions form higher order structures.

A library of YI12 peptides were designed based on the long range packing that was identified between W4 and M9 in YI12 peptide (First generation). Hence in second generation, M9 was replaced with aromatic aminoacids like Phe, Trp and Tyr to make the packing intimate and enhanced. SAR studies of second generation of peptides in YI12 library revealed that the antimicrobial and antiendotoxic activity were governed by balance between cationic residues and conserved long range aromatic packing 
[150]. It was found that at very high concentration of endotoxin units (8 EU/ml) also, YI12WF, YI12WW and YI12WY peptides demonstrated $80 \%$ of LPS neutralization activity [150]. YI12WF peptide was chosen for acylation studies because of the close packing between W5 and F9 and superior activities.

In the third generation, strategies like acylation and dimerization using disulfide bridges were tested on YI12WF. Because of increased hydrophobicity, acylated C4YI12WF and C8YI12WF, cysteine dimerized C4YI12WFC and C8YI12WFC remained insoluble in buffers. Hence a lysine residue was added in KRKR motif to improve solubility of acylated and dimerized peptides. Inclusion of lysine residues were known to increase solubility of peptides in water [155]. Hence in the third generation, to test acylation strategy alone, isoleucine analogs, YI13WF, C4YI13WF and C8YI13WF (Table 3.1-1) were designed. To test acylation along with disulfide bond strategy, cysteine analogs, YI13WFC, C4YI13WFC and C8YI13WFC were designed (Table 3.1-1). Alanine mutation of W4 and F10 was also included with acylation and disulfide bond to examine the aromatic packing importance.

Table 3.1-1: Third generation of $\beta$-boomerang peptides and their primary structure.

\begin{tabular}{|l|l|}
\hline Peptide & Aminoacid sequence \\
\hline Isoleucine analogs & \\
\hline YI13WF & Y-V-L-W-K-R-K-R-K-F-I-F-I \\
\hline C4YI13WF & C4-Y-V-L-W-K-R-K-R-K-F-I-F-I \\
\hline C8YI13WF & C8-Y-V-L-W-K-R-K-R-K-F-I-F-I \\
\hline C8YI13AAI & C8-Y-V-L-A-K-R-K-R-K-A-I-F-I \\
\hline Cysteine analogs & \\
\hline YI13WFC & Y-V-L-W-K-R-K-R-K-F-C-F-I \\
\hline C4YI13WFC & C4-Y-V-L-W-K-R-K-R-K-F-C-F-I \\
\hline C8YI13WFC & C8-Y-V-L-W-K-R-K-R-K-F-C-F-I \\
\hline C8YI13AAC & C8-Y-V-L-A-K-R-K-R-K-A-C-F-I \\
\hline
\end{tabular}




\subsubsection{Dimerization of cystiene containing peptides and HPLC purification:}

The cysteine analogs were oxidized with 20\% DMSO and further purified with RPHPLC. When the crude peptides were passed through HPLC, it yielded one major peak for monomer or completely reduced form of the peptide. After oxidation with $20 \%$ DMSO for 6 hours, there were 2 peaks indicating a mixture of reduced and oxidized forms (Figure 3.1-1). Therefore the oxidation period was extended to 18 hours to purify only the oxidized form.

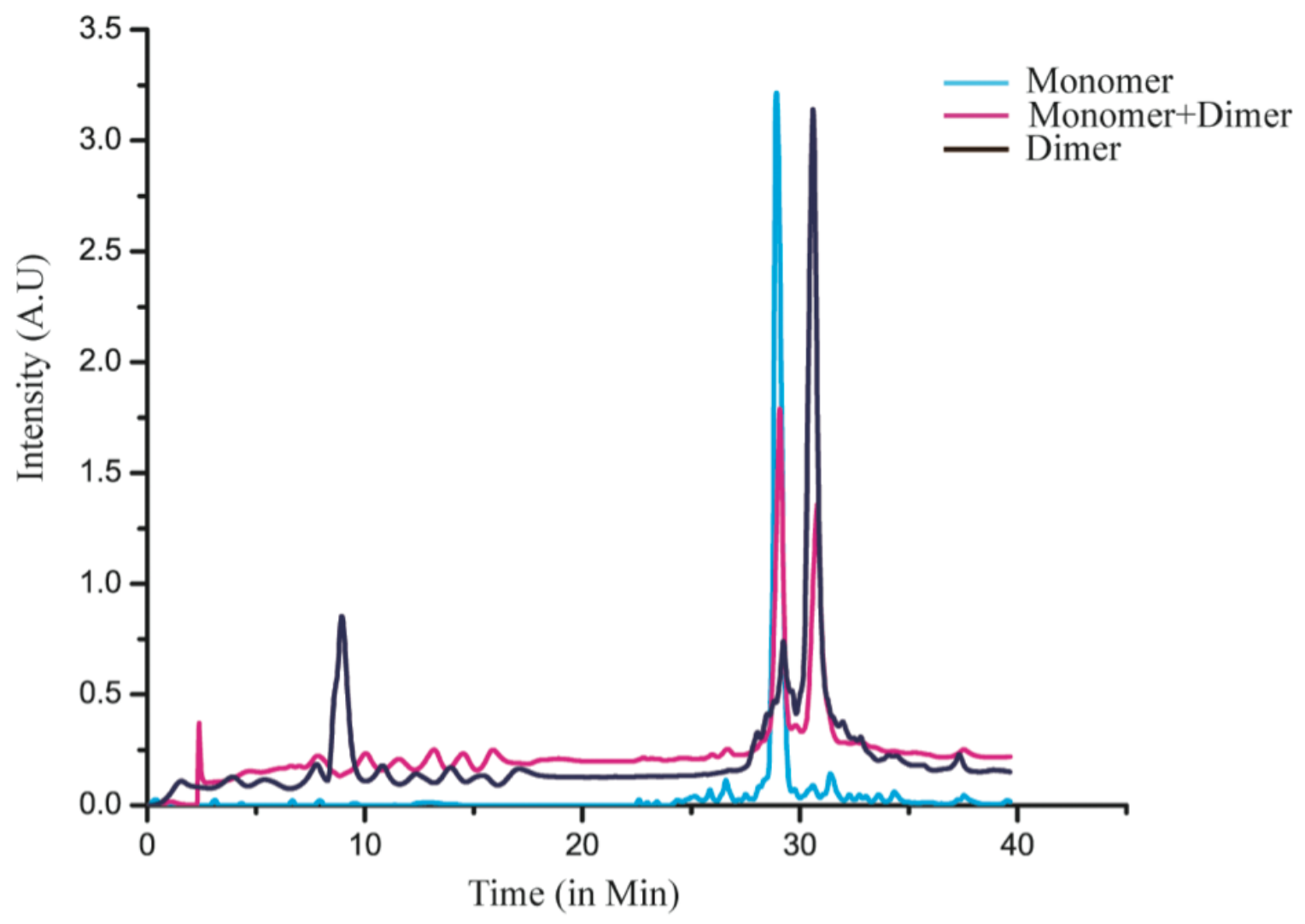

Figure 3.1-1: A representative HPLC purification profile of a cysteine containing peptide. Blue curve represents the crude peptide, pink curve for 6 hours of oxidation and black curve for 18 hours of oxidation.

The purified peptides were then freeze dried under vacuum for 16 hours and the lyophilized peptides are used for the further experiments. Table 3.1-2 shows retention time of the isoleucine and oxidized cysteine analog peptides in C18 HPLC column. A longer retention time was observed for the oxidized form of the peptides indicating higher hydrophobicity. 
Table 3.1-2: Retention time of the peptides purified using HPLC.

\begin{tabular}{|l|l|}
\hline Peptide & Retention time $\left(\mathrm{R}_{\mathrm{t}}\right)$ \\
\hline YI13WF & 23.60 \\
\hline C4YI13WF & 30.43 \\
\hline C8YI13WF & 33.98 \\
\hline C8YI13AAI & 30.87 \\
\hline YI13WFC & 27.48 \\
\hline C4YI13WFC & 31.62 \\
\hline C8YI13WFC & 37.40 \\
\hline C8YI13AAC & 32.38 \\
\hline
\end{tabular}

\subsubsection{Activity assays:}

\subsubsection{Determination of Minimum Inhibitory Concentration (MIC):}

The ability of the designed analogs to kill Gram negative and Gram positive bacterial strains were analysed in $10 \mathrm{mM}$ sodium phosphate buffer. MIC assays demonstrated that the incorporation of inter-molecular disulfide bond and acylation exerts strong bactericidal effects on all the gram-negative and gram-positive strains (Table 3.1-3, Table 3.1-4).

Significantly large differences in MIC values can be seen between Y13WFC and YI13WF peptides (Table 3.1-3, Table 3.1-4). YI13WF appeared to be rather inactive (MICs $\geq 200 \mu \mathrm{M}$ ), except for E. coli, by contrast, YI13WFC showed a much higher bacterial sterilization (Table 3.1-3). In other words, inclusion of the disulfide bond had rendered peptide active against bacterial strains. The bactericidal activities of acylated analogs $\mathrm{C} 4, \mathrm{C} 8$, of YI13WFC, found to be improved with C8YI3WFC showing lowest MIC values (Table 3.1-3). It is noteworthy that bactericidal activities of the peptides are somewhat strain specific as higher lethal effects were observed against gramnegative strains in comparison to gram positive ones (Table 3.1-3). Acylation has also improved anti-bacterial activities of non-disulfide bonded peptides, however with a 
lower extent. Substitution of aromatic residues W, F, with Ala had yielded rather inactive peptides (Table 3.1-3, Table 3.1-4).

Table 3.1-3: Minimum inhibitory concentration (in $\mu M$ ) of Isoleucine and Cysteine analogs in Gram negative bacteria.

\begin{tabular}{|l|l|l|l|l|}
\hline Peptide & E.coli & $\begin{array}{l}\text { P.aeruginosa } \\
\text { ATCC27853 }\end{array}$ & $\begin{array}{l}\text { K.pnuemoniae } \\
\text { ATCC13883 }\end{array}$ & $\begin{array}{l}\text { S.enterica } \\
\text { ATCC14028 }\end{array}$ \\
\hline YI13WF & 15 & $>200$ & 150 & $>200$ \\
\hline C4YI13WF & 12 & 60 & 60 & 40 \\
\hline C8YI13WF & 12 & 40 & 40 & 60 \\
\hline C8YI13AAI & 100 & 100 & 200 & $>200$ \\
\hline YI13WFC & 12.5 & 20 & 25 & 50 \\
\hline C4YI13WFC & 10 & 15 & 8 & 50 \\
\hline C8YI13WFC & 3 & 5 & 12 & 50 \\
\hline C8YI13AAC & 50 & 100 & 100 & $>200$ \\
\hline
\end{tabular}

Table 3.1-4: Minimum inhibitory concentration (in $\mu M$ ) of Isoleucine and Cysteine analogs in Gram positive bacteria.

\begin{tabular}{|l|l|l|l|l|}
\hline Peptide & B.subtilis & $\begin{array}{l}\text { S.aureus } \\
\text { ATCC25923 }\end{array}$ & $\begin{array}{l}\text { S.pyogenes } \\
\text { ATCC19615 }\end{array}$ & $\begin{array}{l}\text { E.faecalis } \\
\text { ATCC29212 }\end{array}$ \\
\hline YI13WF & 200 & 200 & $>200$ & $>200$ \\
\hline C4YI13WF & 80 & 60 & 60 & 60 \\
\hline C8YI13WF & 60 & 8 & 60 & 40 \\
\hline C8YI13AAI & 100 & 200 & $>200$ & $>200$ \\
\hline YI13WFC & 20 & 20 & 50 & 50 \\
\hline C4YI13WFC & 15 & 50 & 50 & 4 \\
\hline C8YI13WFC & 5 & 5 & 50 & 50 \\
\hline C8YI13AAC & 50 & 200 & $>200$ & $>200$ \\
\hline
\end{tabular}


The effects of N-terminal acylation of the peptides were also analyzed. Acylation of AMPs improved antimicrobial and antifungal activity [156]. The acylated analogues of the designed peptides showed marked increase in antibacterial activity when compared to its nonacylated analogs. The isoleucine analogs have increased MIC when compared to the second generation YI12WF peptide. But when the isoleucine is replaced with cysteine to form dimers, it increases the antibacterial activity by approximately two fold.

Thus the MIC data clearly suggest that the mere increase in hydrophobicity and basicity alone cannot lead to better design of anti-microbial peptides. There is need for specific interaction between the residues. The cysteine analogs can inhibit the growth of bacteria more effectively when compared to its isoleucine analogs. But the presence of disulfide bond alone is not sufficient for the peptides to exert its killing activity as examined with C8AAC.

\subsubsection{Assay for LPS neutralization:}

In order to develop antimicrobial agents against sepsis, the compounds should be highly active in LPS neutralization. Hence the competing ability of the active cysteine analogs to neutralize LPS was assayed using chromogenic LAL kit. LAL assay was performed at three different concentrations of LPS and four different concentrations of peptides.

All of the peptides tested show $80 \%$ neutralization in different concentration of LPS (expressed as endotoxin units, EU), in dose dependent fashion (Figure 3.1-2). The non acylated YI13WFC peptide also shows neutralization activity equal to acylated analogs as the concentration of the peptide increases (Figure 3.1-2, red bars). But the acylated analog C8YI13WFC peptide shows around 60\% neutralization of $0.4 \mathrm{EU}$ at $1.0 \mu \mathrm{M}$ which decreases only slightly to $50 \%$ even the concentration of LPS increased to as high as 1.0 EU (Figure 3.1-2, green bars). It was noteworthy to observe that C4YI13WFC peptide shows around 40\% of 0.4 EU LPS sequestration and as the concentration of LPS increases to $1.0 \mathrm{EU}$, the neutralizing activity also increases to 50\% (Figure 3.1-2, blue bars). The inactive peptide C8YI13AAC, however shows only $30 \%$ neutralization at highest concentration of the peptide tested. 


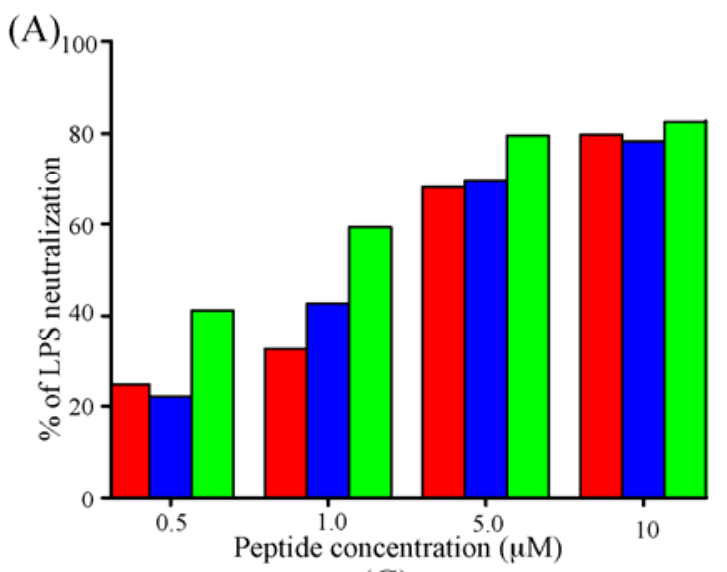

(C)
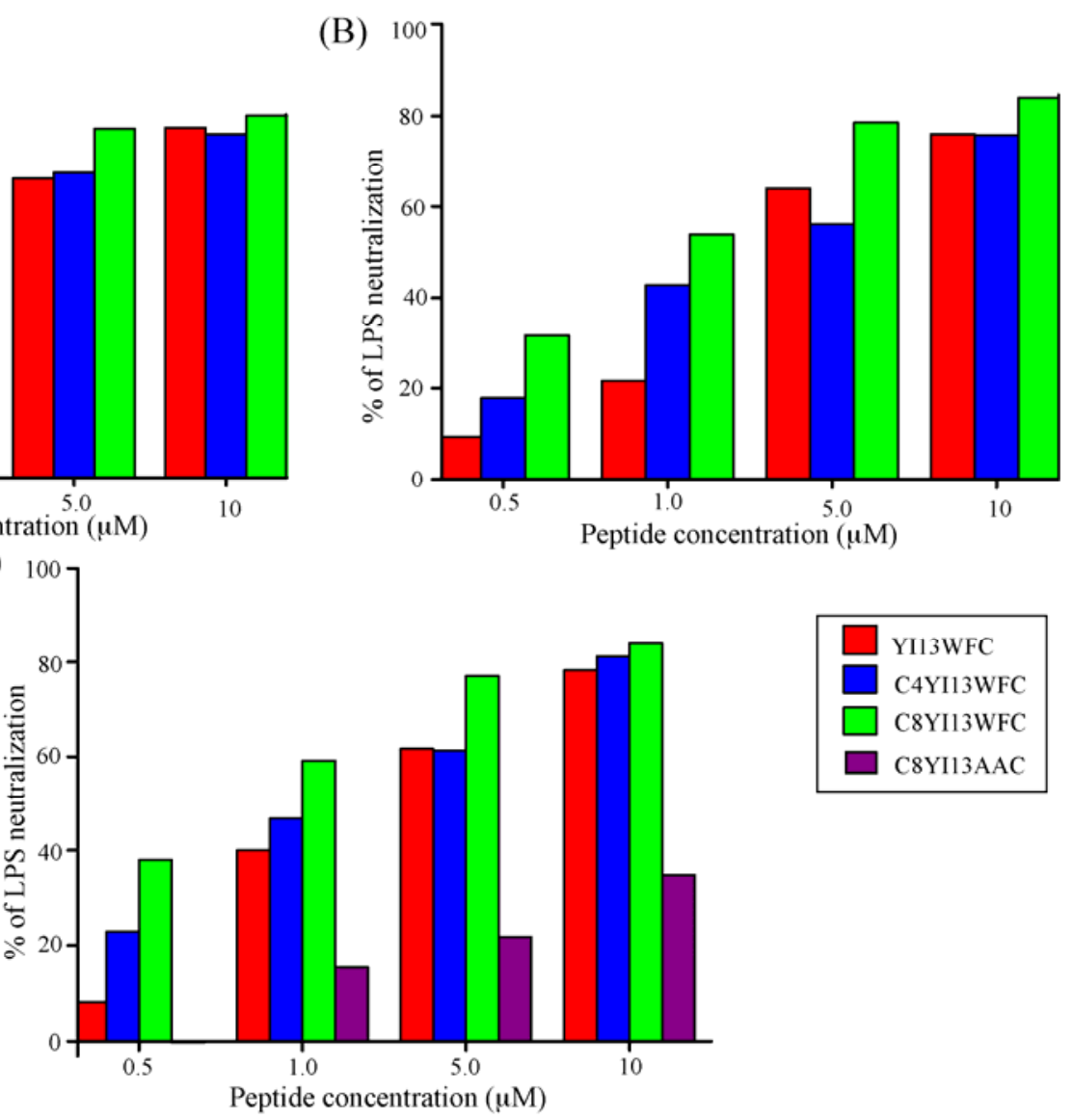

YI13WFC C4YI13WFC C8YI13WFC C8YI13AAC

Figure 3.1-2: Bar diagrams representing the LPS neutralization activity of Cysteine analogs at different EU concentrations, (A) $0.4 \mathrm{EU}$ (B) $0.8 \mathrm{EU}$ and (C) $1.0 \mathrm{EU}$.

\subsubsection{Hemolytic assay:}

The ability of cysteine and isoleucine analogs to differentiate bacterial cells and mammalian cells were assayed using red blood cells. Isoleucine analogs were found to be slightly hemolytic than cysteine analogs (Table 3.1-5). Interestingly acylation of the peptides was found to have positive effect on hemolysis i.e. hemolytic activity decreases with increase in the length of the acyl chain of isoleucine analogs. But in the case of cysteine analogs, hemolysis seems to decrease with $\mathrm{C} 4$ acylation which increases further with C8 acylation (Table 3.1-5). The aromatic aminoacid substitution of both isoleucine (C8YI13AAI) and cysteine (C8YI13AAC) analogs have rendered to be highly hemolytic indicating the specifiticy of aromatic packing (Table 3.1-5). 
Table 3.1-5: Percentage of hemolysis of cysteine and isoleucine analogs at two different concentrations.

\begin{tabular}{|l|l|l|}
\hline Peptides & $\begin{array}{l}\text { \% of hemolysis at } 5 \mu \mathrm{M} \\
\text { peptide concentration }\end{array}$ & $\begin{array}{l}\text { of hemolysis at } 50 \mu \mathrm{M} \\
\text { peptide concentration }\end{array}$ \\
\hline YI13WF & 12.9 & 26.7 \\
\hline C4YI13WF & 6.3 & 13.8 \\
\hline C8YI13WF & 4.9 & 14.5 \\
\hline C8YI13AAI & 44.5 & 60.8 \\
\hline YI13WFC & 8.7 & 21.5 \\
\hline C4YI13WFC & 3.2 & 14.1 \\
\hline C8YI13WFC & 9.2 & 21.5 \\
\hline C8YI13AAC & 16.7 & 30.2 \\
\hline
\end{tabular}

\subsubsection{Surface charge measurements by Zeta potential:}
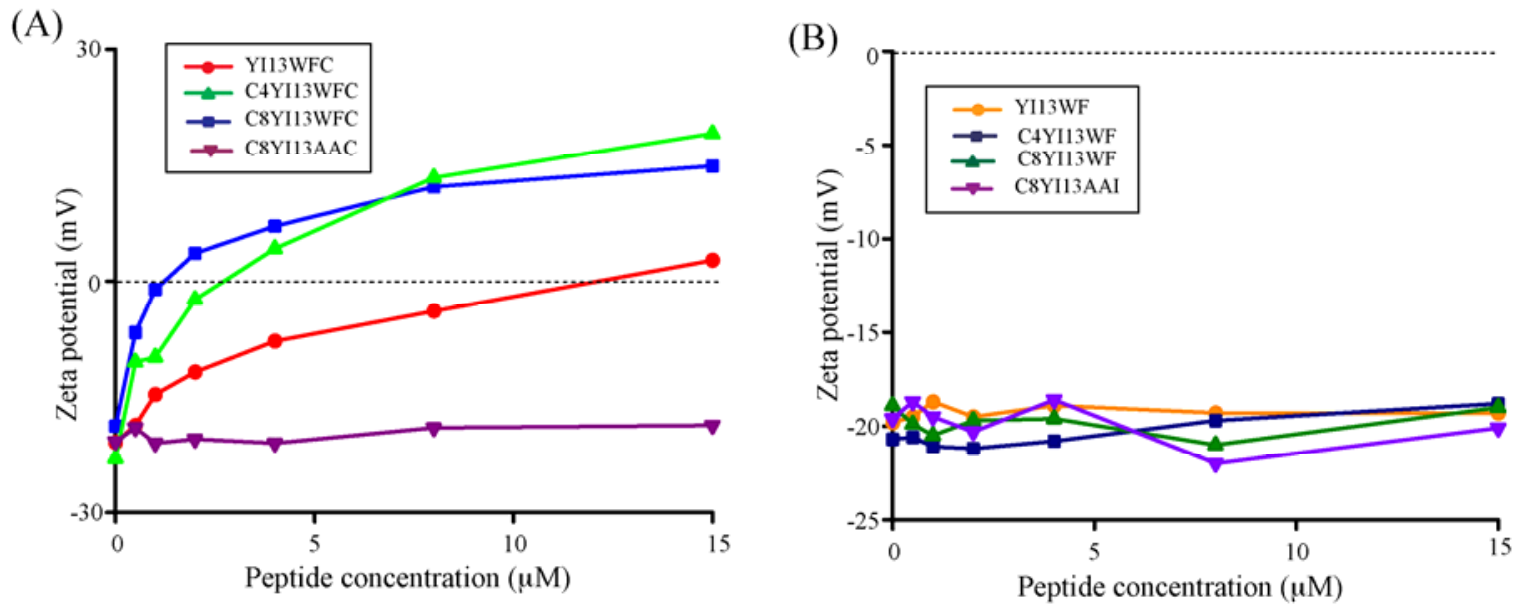

Figure 3.1-3: Surface charge measurement of E.coli cells in the presence of (A) Cysteine analogs and (B) Isoleucine analogs.

Measuring zeta potential assessed surface charge neutralization ability of the peptides. Zeta potential is widely used to quantify the surface charges that are readily available for the AMPs to neutralize and thereby disrupt the cells. Gram negative bacteria 
generally exhibit larger negative potential because of the presence of carboxylate and phosphate groups on the membrane surface.

When E.coli cells were analysed in the absence of peptides, it exhibited a negative potential of about $-20.0 \mathrm{mV}$ (Figure 3.1-3). Addition of increasing concentration of active cysteine analogs effectively neutralized the bacterial cell surface (Figure 3.1-3, A). After addition of $4 \mu \mathrm{M}$ of C4YI13WFC and C8YI13WFC, over compensation of surface charge of about +19.1 and +24.0 was noted respectively. Such over compensation of charges were generally obtained when the peptides are inserted into hydrophobic milieu of the membrane. On the other hand, the inactive C8YI13AAC does not show any change in zeta potential. The same was observed for all isoleucine analogs (Figure 3.1-3, B). This clearly indicates that the acylation with dimerization of the peptides can effectively interact with hydrophobic environment of the bacterial cells and destroy them.

\subsubsection{Biophysical Characterisation:}

\subsubsection{Outer membrane permeability:}
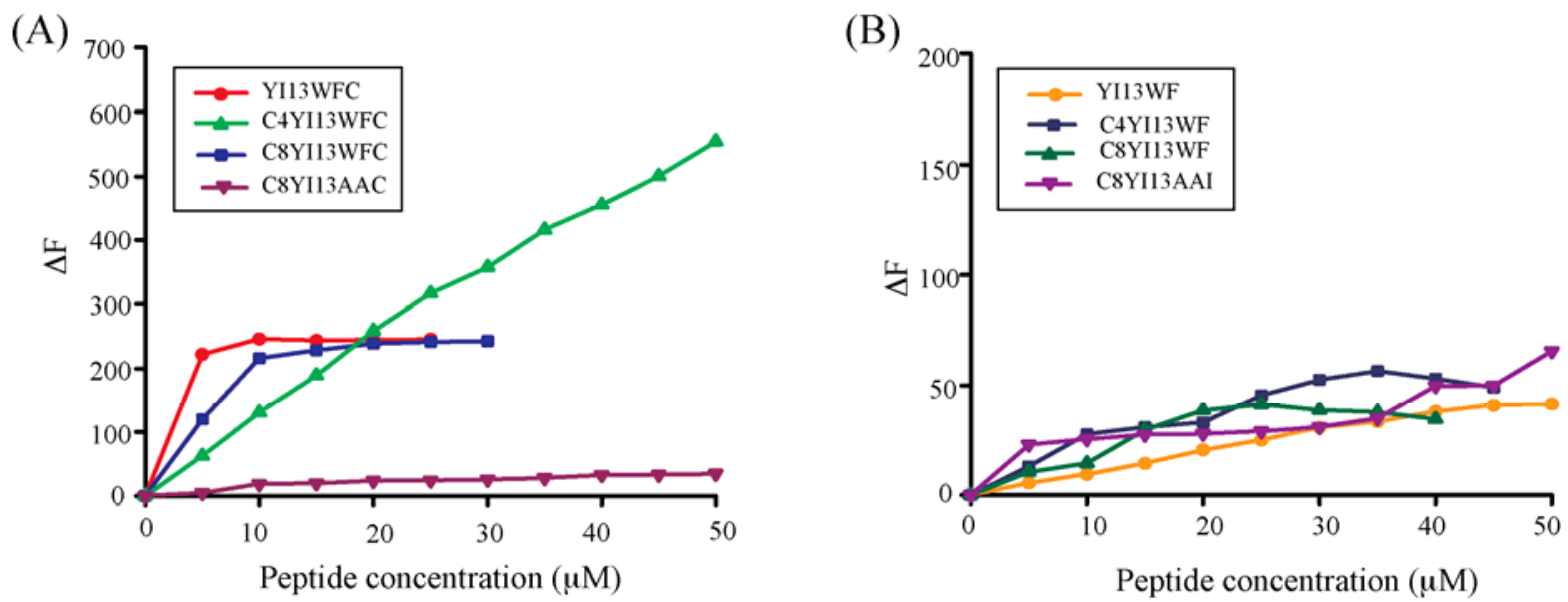

Figure 3.1-4: Permeability of fluorescent probe, NPN caused by the disruption of outer membrane of E.coli cells by (A) Cysteine analogs and (B) Isoleucine analogs.

The challenging ability of cysteine and isoleucine analogs to permeabilize through the outer membrane of E.coli cells was assayed with the help of fluorescent probe, NPN. 
1-N-phenylnapthylamine cannot partition into intact cells and has very weak fluorescence in aqueous environment. If the integrity of outer membrane is perturbed, then NPN can partition into hydrophobic environment and fluoresces.

The ability to permeabilize outer membrane was dose dependent i.e. as the concentration of peptide increases; the fluorescent intensity of NPN also increases for cysteine analogs (Figure 3.1-4, A). At around $10 \mu \mathrm{M}$ of YI13WFC and C4YI13WFC, the intensity reaches a plateau, whereas it continues to increase for C8YI13WFC. There is no marked increase in intensity for inactive C8YI13AAC peptide (Figure 3.1-4, A). Irrespective of acylation and presence of aromatic aminoacids, the isoleucine analogs do not show marked increase in fluorescent intensity (Figure 3.1-4, B).

\subsubsection{Intrinsic tryptophan fluorescence:}

Tryptophan is an excellent fluorescent probe to monitor the interactions of the peptides with lipids and detergent micelles like LPS, SDS and DPC. The intrinsic fluorescence of the free peptide shows an emission maximum around $354 \mathrm{~nm}-358 \mathrm{~nm}$ indicating maximum exposure of Trp residue to the aqueous environment. The emission maxima of Trp were drastically changed to $334 \mathrm{~nm}-339 \mathrm{~nm}$ upon additions of increasing concentrations of LPS micelles (Figure 3.1-5, A).

The presence of blue shift i.e. shift of the emission maximum towards shorter wavelengths is an indication of the incorporation of the peptides in to hydrophobic environment of the micelles [149]. The extent of blue shift is noticed to be higher for C4-acylated analogues with and without disulfide bond followed by nonacylated and C8-acylated analogues (Figure 3.1-5, A, B). The possible explanation of C8-acylated analogues having least blue shift could be the presence of long hydrophobic acyl chain that may hinder the insertion of the tryptophan into the hydrophobic environment. C4acylated analogues exhibit maximum blue shift because of the optimum acyl chain length and hence the threshold hydrophobicity needed for the insertion of the peptides is easily achieved. 

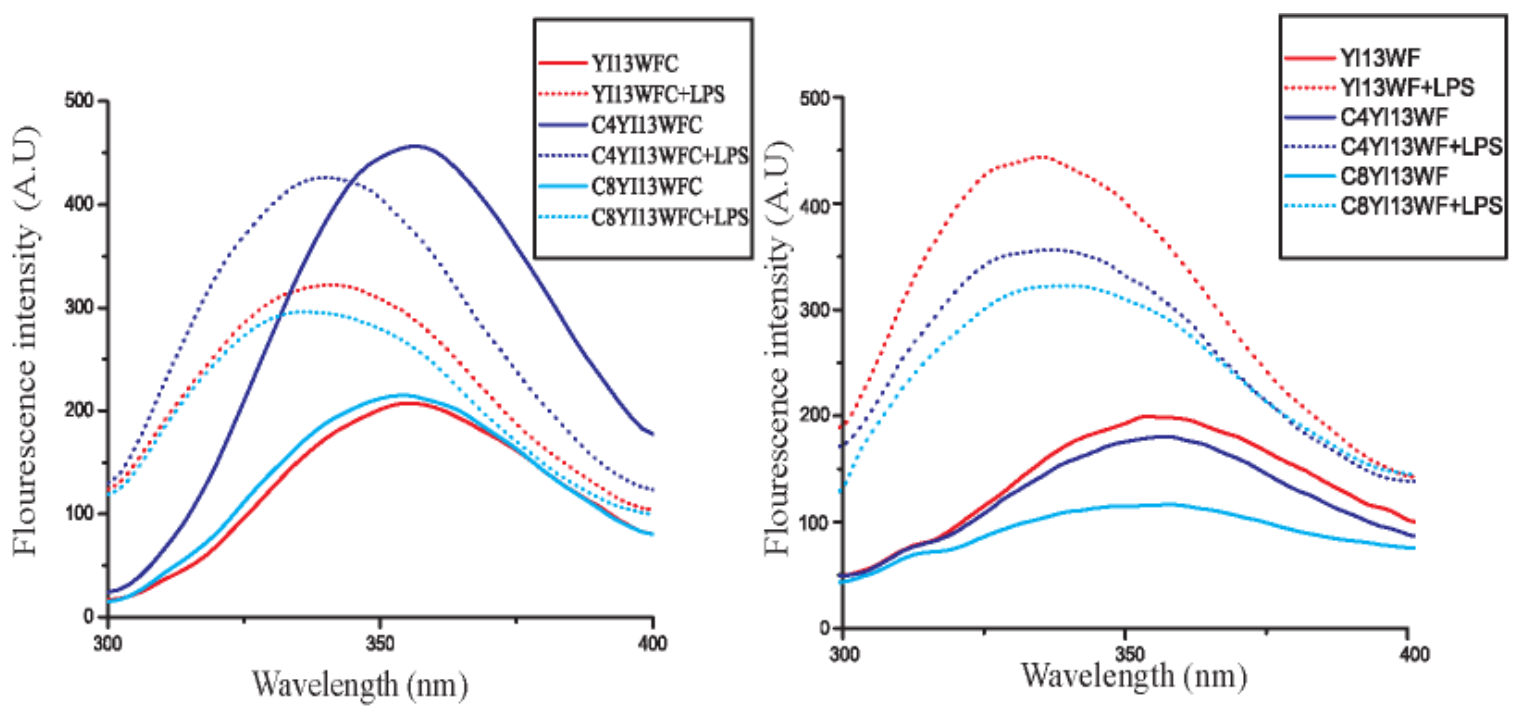

Figure 3.1-5: Intrinsic tryptophan fluorescence measurement of cysteine and isoleucine analogs in buffer (colored straight lines) and in LPS micelles (colored dotted lines).

Table 3.1-6: Difference in the emission maximum of Trp fluorescence ( $\Delta \lambda \max )$ in the aqueous environment and in LPS and DPC micelles.

\begin{tabular}{|l|l|l|}
\hline \multirow{2}{*}{ Peptide } & \multicolumn{2}{|l|}{$\Delta \lambda \max$} \\
\cline { 2 - 3 } & LPS & DPC \\
\hline YI13WF & 19 & 8 \\
\hline C4YI13WF & 20 & 16 \\
\hline C8YI13WF & 18 & 6 \\
\hline YI13WFC & 17 & 8 \\
\hline C4YI13WFC & 20 & 8 \\
\hline C8YI13WFC & 12 & 14 \\
\hline
\end{tabular}

A restricted blue shift of Trp was observed in the presence of zwitterionic DPC micelles (Table 3.1-6). The C4YI13WFC peptide that exhibited maximum blue shift in LPS, demonstrated a lowest blueshift with DPC micelles (Table 3.1-6). This indicates that the interaction of the peptides with DPC micelles is limited to the surface and the peptide is lipid selective. The C8-acylated analogues interacted with DPC indicating the loss of membrane selectivity. 


\subsubsection{Acrylamide quenching:}

Quenching of Trp fluorescence of the free peptide and peptide-LPS, peptide-DPC complexes with neutral quencher, acrylamide, further assessed the extent of incorporation of the fluorophore into hydrophobic environment of lipids. The exposure of Trp residue to the quencher is maximum when it is exposed and minimum when it is buried inside the hydrophobic environment. Table 3.1-7 shows the SternVolmer constants of the peptides in free and in complexes with LPS and DPC. As can be seen, the free peptide has a very high $\mathrm{K}_{\mathrm{sv}}$ value indicating a maximum exposure of the tryptophan residue to the quencher. In the presence of LPS, the quenching constant values decreased drastically demonstrating that the tryptophan residue is not solvent exposed and buried inside LPS micelles.

Table 3.1-7: Quenching of tryptophan residues of the peptides by acrylamide.

\begin{tabular}{|l|l|l|l|}
\hline Peptide & $\mathrm{K}_{\mathrm{sv}}($ Free $)$ & $\mathrm{K}_{\mathrm{sv}}(\mathrm{LPS})$ & $\mathrm{K}_{\mathrm{sv}}(\mathrm{DPC})$ \\
\hline YI13WF & 32.8 & 5.0 & 11.8 \\
\hline C4YI13WF & 30.7 & 5.5 & 9.3 \\
\hline C8YI13WF & 12.6 & 5.6 & 4.6 \\
\hline YI13WFC & 43.1 & 6.0 & 11.1 \\
\hline C4YI13WFC & 37.9 & 5.8 & 11.3 \\
\hline C8YI13WFC & 23.3 & 5.0 & 11.2 \\
\hline
\end{tabular}

In the presence of DPC also the tryptophan residue is not solvent exposed which is indicated by decrease in $\mathrm{K}_{\mathrm{sv}}$ values (Table 3.1-7). But the values are two times more when compared to LPS, indicating that the peptides are membrane selective and the interaction with DPC micelles could be limited to the surface of the membrane and not penetrating into the membrane. 


\subsubsection{Isothermal titration calorimetry:}

(A)

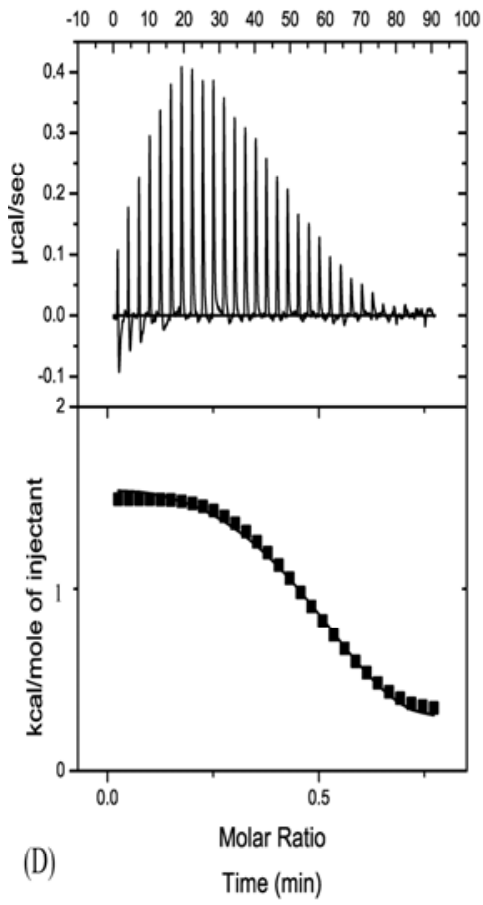

$\begin{array}{rrrrrrrrrrrr}-10 & 0 & 10 & 20 & 30 & 40 & 50 & 60 & 70 & 80 & 90 & 100\end{array}$

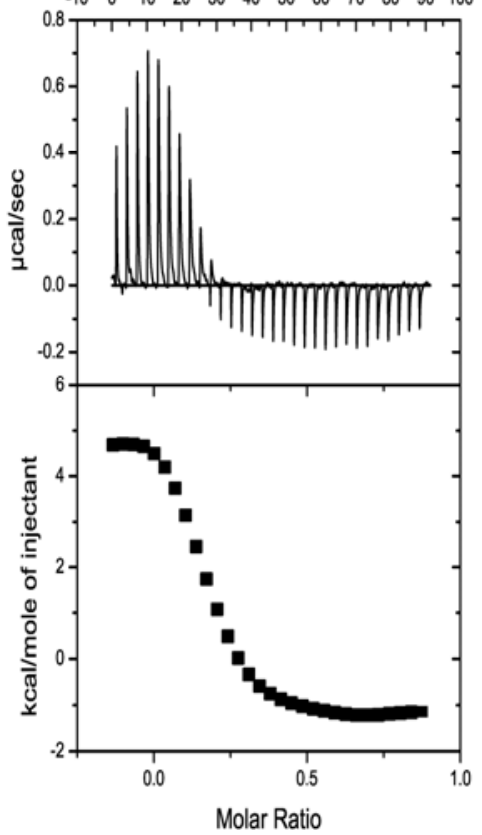

(B)
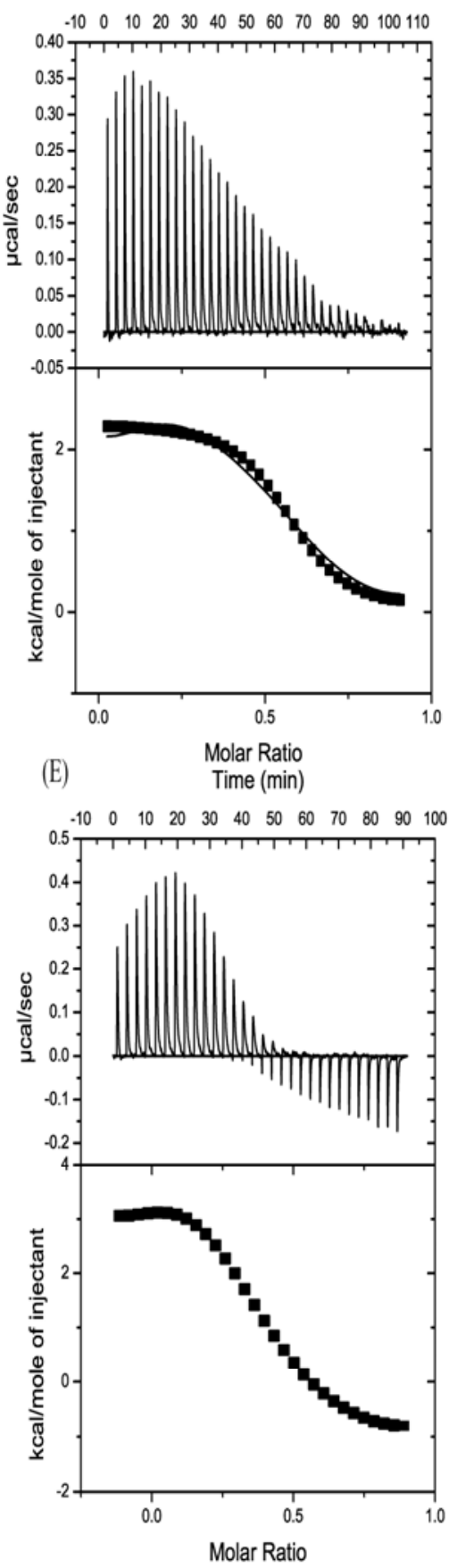

(C)
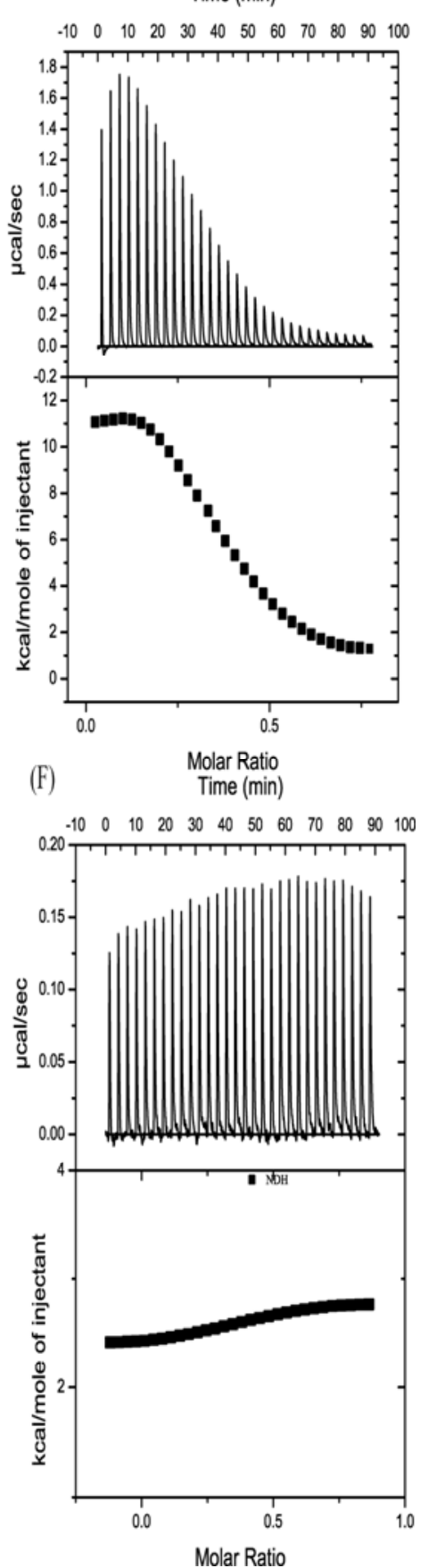

Figure 3.1-6: Binding profiles of isoleucine analogs (A) YI13WF, (B) C4YI13WF, (C) C8YI13AAI and cysteine analogs (D) YI13WFC, (E) C4YI13WFC, (F) C8YI13AAC with LPS micelles at $25^{\circ} \mathrm{C}$ by Isothermal titration calorimetry. 
The binding affinities of the cysteine analogs and isoleucine analogs with LPS micelles were determined using ITC. Thermodynamic parameters were obtained for all of the peptides except C8YI13WF and C8YI13WFC as the signal to noise ratio was poor. The binding of the peptides with LPS micelles was found to be endothermic reactions since the titrations generated upward peaks. As can be seen from Figure 3.1-6, all peptides were found to interact with LPS micelles except C8YI13AAC.

The thermodynamic parameters were derived by fitting the data. It was noted that the $\Delta \mathrm{G}$ values decreases considerably between acylated cysteine and isoleucine analogs when compared to their nonacylated counterparts that demonstrates a favorable binding. Further the dimerization of the peptides also has enhanced hydrophobicity, which is clearly indicated by the decrease in $\Delta \mathrm{G}$ values of cysteine analogs (Table 3.1-8).

Table 3.1-8: Thermodynamic parameters of cystiene and isoleucine analogs binding to LPS micelles determined by Isothermal titration calorimetry.

\begin{tabular}{|l|l|l|l|l|l|}
\hline & YI13WF & C4YI13WF & YI13WFC & C4YI13WFC & C8YI13AAI \\
\hline $\mathrm{K}_{\mathrm{a}}\left(\mu \mathrm{M}^{-1}\right)$ & 0.36 & 0.86 & 4.2 & 2.2 & 0.29 \\
\hline$\Delta \mathrm{H}\left(\mathrm{kcal}_{\mathrm{mol}}{ }^{-1)}\right.$ & 5.6 & 2.3 & 4.6 & 3.0 & 1.2 \\
\hline $\begin{array}{l}\mathrm{T} \Delta \mathrm{S} \mathrm{(kcal.mol} \\
\left.{ }^{-1 e \mathrm{de}^{-1}}\right)\end{array}$ & 13.1 & 10.3 & 13.6 & 11.6 & 8.6 \\
\hline$\Delta \mathrm{G}\left({\left.\mathrm{kcal} . \mathrm{mol}^{-1}\right)}^{-}\right.$ & -7.5 & -8.09 & -9.03 & -8.6 & -7.4 \\
\hline $\mathrm{K}_{\mathrm{d}}(\mu \mathrm{M})$ & 2.7 & 1.1 & 0.23 & 0.45 & 3.45 \\
\hline
\end{tabular}

N-terminal acylation also improves the binding affinity of the peptides to bacterial membranes [157]. In the case of cysteine and isoleucine analogs, acylation and dimerization greatly enhances the binding affinity of the peptides. As indicated in Table 3.1-8, the cysteine analogs exhibit greater binding affinity of $0.23 \mu \mathrm{M}$ for YI13WFC and $0.45 \mu \mathrm{M}$ for C4YI13WFC. On the other hand, the isoleucine analogs exhibited fairly higher $\mathrm{K}_{\mathrm{d}}$ values with the control peptide C8AAI exhibiting $3.4 \mu \mathrm{M}$ which explains that mere LPS binding property does not correlate with LPS neutralization and antimicrobial activity. 


\subsubsection{Dissociation of FITC-LPS aggregates:}

The marked difference in outer membrane permeability was further assessed utilizing FITC-LPS fluorescence. The outer layer of outer membrane of Gram negative bacterial cells are composed of a unique lipid named Lipopolysaccharide (LPS). It serves as permeability barrier and helps to maintain the cells shape and structure. FITC fluorescence is highly quenched in FITC-LPS micelles due to close proximity of chromophores. Dissociation of LPS aggregates causes dequenching of FITC fluorescence.

(A)

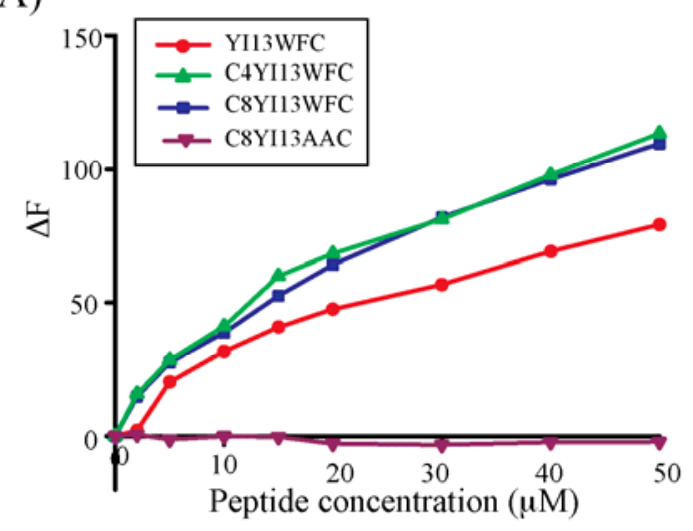

(B)

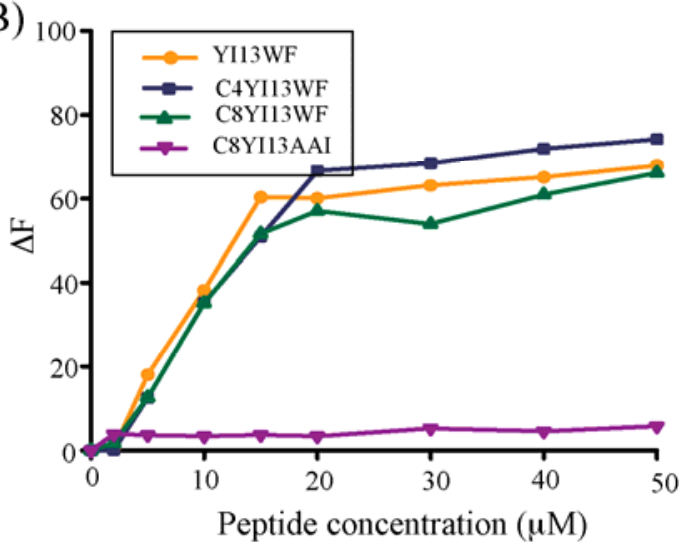

Figure 3.1-7: Dequenching of FITC fluorescence in FITC-LPS by (A) Cysteine analogs and (B) Isoleucine analogs.

In parallel with outer membrane permeability assay, the cysteine analogs showed increase in fluorescent intensity as the concentration of peptide increases. Acylated cysteine analogs were found to have slightly higher dissociating ability than YI13WFC peptide (Figure 3.1-7, A). The fluorescent intensity remained unchanged even when the concentration of inactive C8YI13AAC increased (Figure 3.1-7, A). Dequenching of FITC fluorescence was also observed with isoleucine analogs (Figure 3.1-7, B) but there was marked difference in the intensity changes between cysteine and isoleucine analogs (Figure 3.1-7). It should also be noted that LPS dissociating ability of cysteine analogs increases with increase in acylation whereas it decreases in isoleucine analogs. Collectively, all these results suggest that increase in 
hydrophobicity alone cannot develop an effective anti-infective agent. But specific packing with hydrophobicity can be taken as an alternative in developing such agents.

\subsubsection{Dynamic light scattering measurements:}

To further validate the ability of the peptides to dissociate LPS aggregates, dynamic light scattering measurements were carried out. In the absence of peptides, LPS displayed a wide distribution of particles ranging from 5.0 to 50,000 nm (Figure 3.1-8, A) with $814 \mathrm{~nm}$ sized aggregates dominating (Table 3.1-9).
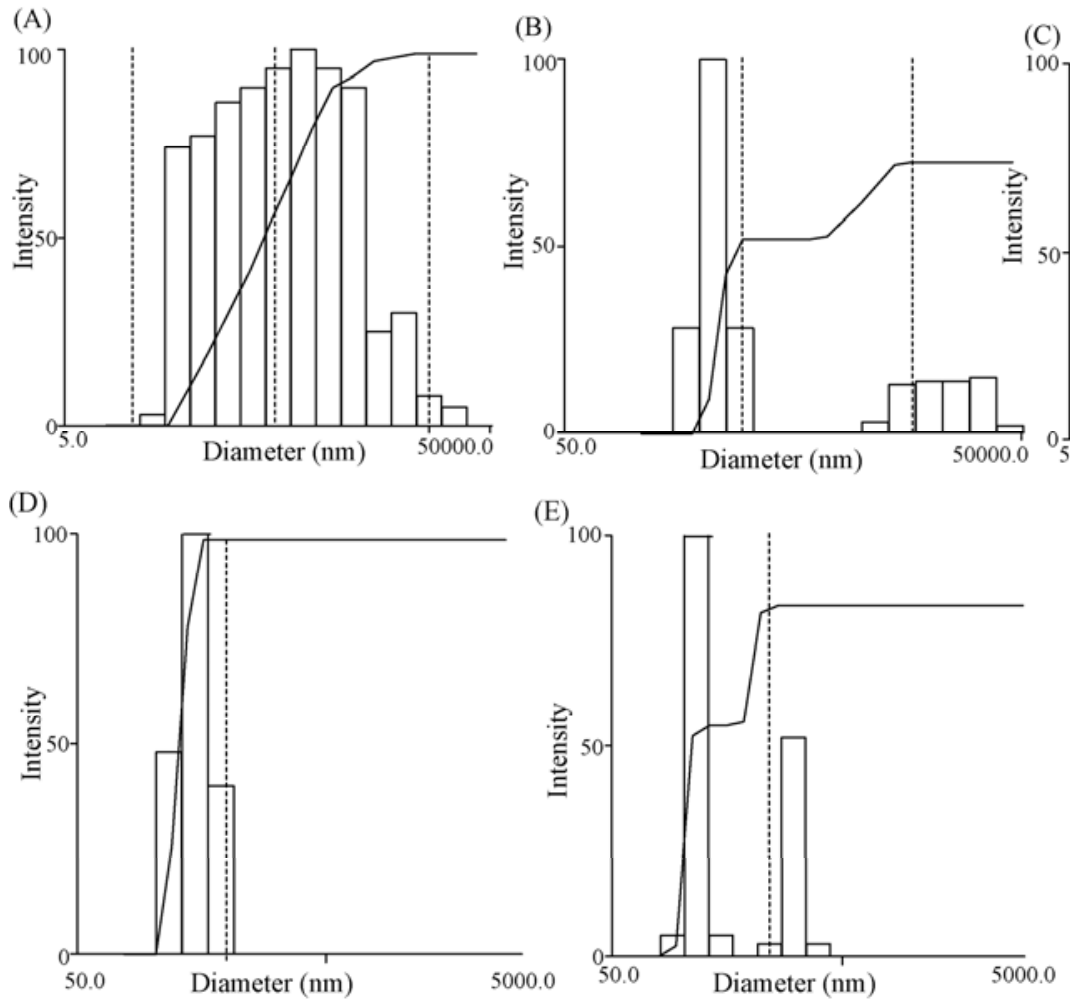

Figure 3.1-8: Dynamic light scattering measurements of (A) LPS, (B) LPS: C4YI13WF (1:4), (C) LPS: C8YI13WF (1:4), (D) LPS: C4YI13WFC (1:4) AND (E) LPS: C8YI13WFC (1:4).

When isoleucine analogs were added to LPS in increasing concentrations, only nonacylated YI13WF could dissociate LPS micelles but only to $500 \mathrm{~nm}$ (Table 3.1-9). The acylated isoleucine analogs could not perturb LPS and further aggregated along with LPS (Figure 3.1-8 A, B). But with the addition of increasing concentration of cysteine analogs, both acylated and nonacylated analogs showed dissociation of larger aggregates to smaller particles. At the ratio of 1:4, only smaller aggregates of diameter 
291, 331 and $385 \mathrm{~nm}$ were present for YI13WFC, C4YI13WFC and C8YI13WFC respectively (Table 3.1-9). The distribution of LPS micelles also decreased from 5.0$50000 \mathrm{~nm}$ to $50.0-5000 \mathrm{~nm}$ (Figure 3.1-8 D, E). Altogether these results indicate that dimerization along with acylation has proven to be an effective strategy in the development of antiendotoxic antimicrobial peptides.

Table 3.1-9: Dissociation of aggregates of LPS micelles to smaller particles by hybrid peptides.

\begin{tabular}{|l|l|}
\hline LPS:Peptide & Diameter $(\mathrm{nm})$ \\
\hline LPS & 814 \\
\hline LPS: YI13WF (1:4) & 500 \\
\hline LPS: C4YI13WF (1:4) & 1056 \\
\hline LPS: C8YI13WF (1:4) & 1081 \\
\hline LPS: YI13WFC (1:4) & 291 \\
\hline LPS: C4YI13WFC (1:4) & 331 \\
\hline LPS: C8YI13WFC (1:4) & 385 \\
\hline
\end{tabular}

\subsubsection{Structural Characterisation:}

\subsubsection{Solution NMR spectroscopy of representative cysteine and isoleucine analogs in aqueous medium:}

The solution conformations of the selected acylated and nonacylated Cysteine and Isoleucine analogs in their free states were examined using two-dimensional ${ }^{1} \mathrm{H}-{ }^{1} \mathrm{H}$ NOESY and TOCSY experiments. Among the samples screened at different $\mathrm{pH}$ and temperature, the samples at $\mathrm{pH} 3.5$ and temperature at $278 \mathrm{~K}$ exhibited high quality NMR spectra. At lower $\mathrm{pH}$, the exchange between the amide hydrogen atoms of the peptide backbone and the solvent water decreases and hence the quality of 2D spectra improves [158]. The 1-D spectra of the peptide showed good signal dispersion and the same conditions were used to acquire TOCSY and NOESY spectra. 


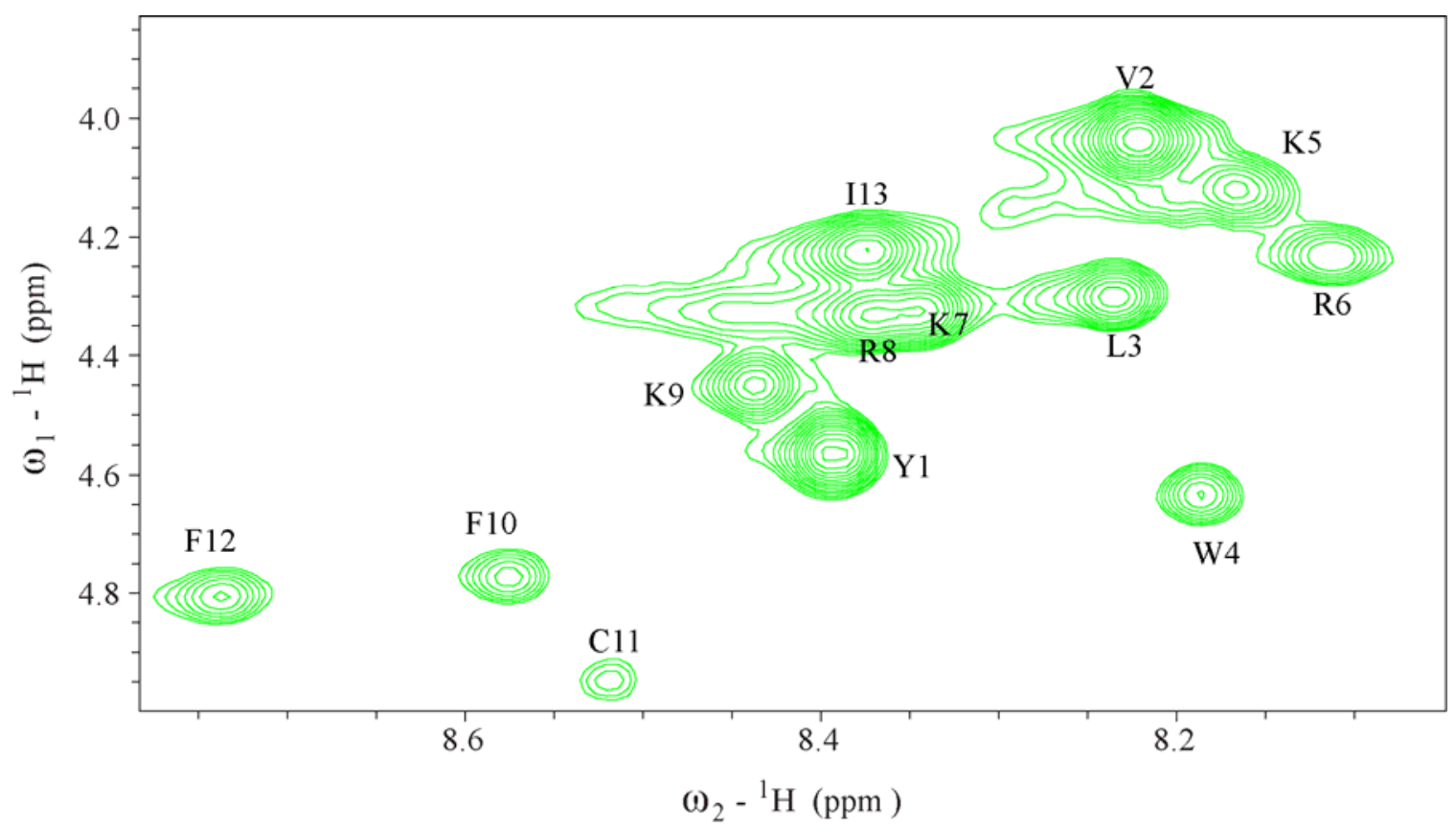

Figure 3.1-9: Sequence specific resonance assignment in the finger print region of two dimensional ${ }^{1} \mathrm{H}-{ }^{1} \mathrm{H}$ TOCSY spectrum of C4YI13WFC in aqueous media at $278 \mathrm{~K}$.

The two dimensional ${ }^{1} \mathrm{H}-{ }^{1} \mathrm{H}$ TOCSY spectrum of C4YI13WFC clearly showed single set of signals for the residues from Tyr1 to Ile13 without any overlap (Figure 3.1-9). Analyses of the TOCSY and NOESY spectra of C4YI13WFC spectra revealed most of the backbone $\mathrm{HN} / \mathrm{HN}$ NOEs. The second generation YI12 peptides were characterized by the NOE contacts of the aromatic lock between W4 and X9 (X is Phe in YI12WF, Trp in YI12WW and Tyr in YI12WY) upon complex with LPS. There were more medium range NOEs of type $\mathrm{HN} / \mathrm{NH}$ and $\mathrm{C} \alpha \mathrm{H} / \mathrm{HN}$ of $i$ to $i+2$ for the LPS binding motif KRKR region. This has imparted an amphipathic structure to the peptides in the presence of LPS.

Interestingly, C4YI13WFC, in the aqueous solution (in absence of LPS) has shown long range NOE contacts. There was an NOE cross peak between the indole ring protons of tryptophan (W4HE1) and beta protons of phenylalanine (F12C $3 \mathrm{H})$ (Figure 3.1-10, C). Further analyses of the spectra also revealed the presence of cross peaks between W4 aromatic side chains with F12 beta protons (Figure 3.1-10, B). 

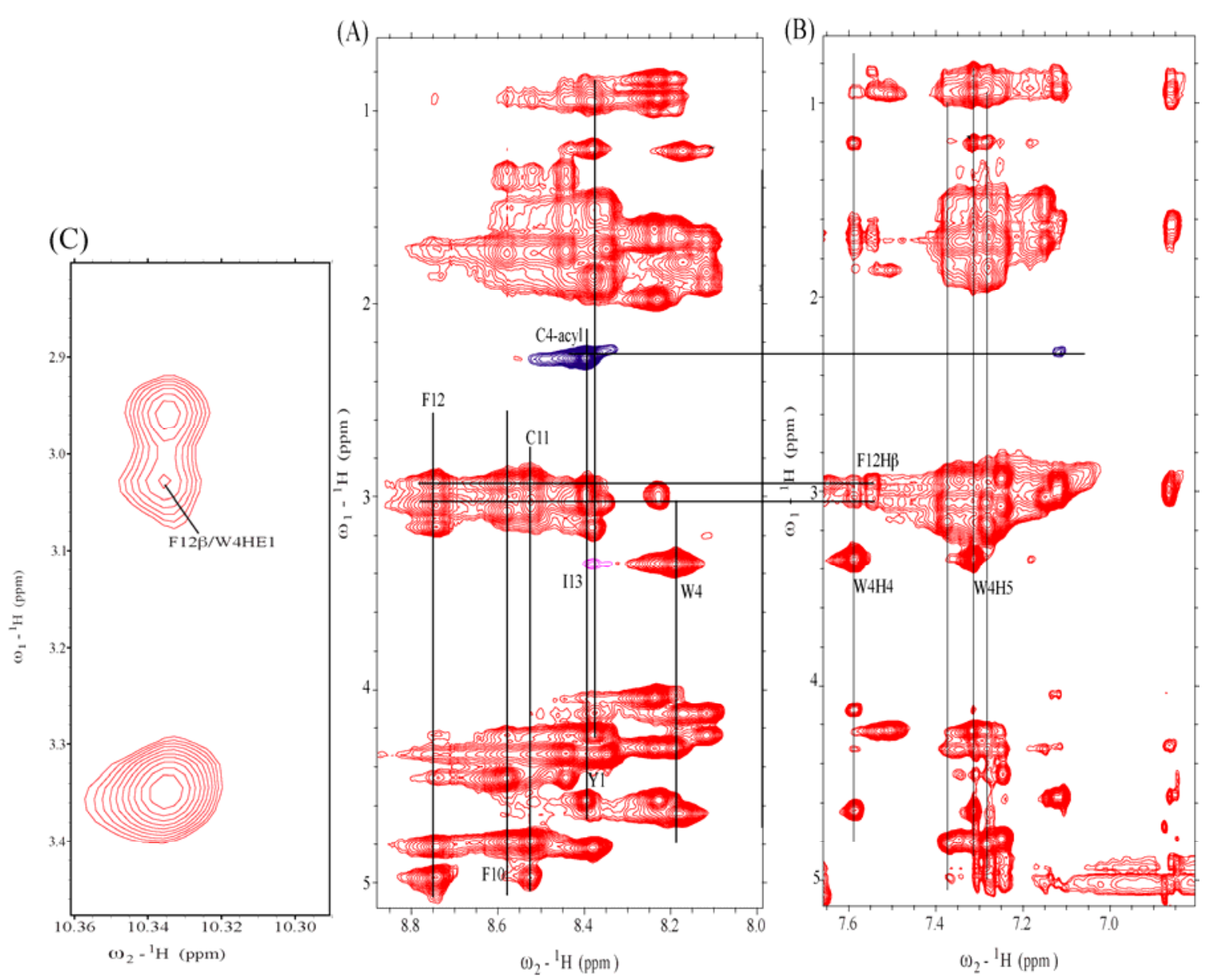

Figure 3.1-10: Selected regions of two-dimensional ${ }^{1} \mathrm{H}-{ }^{1} \mathrm{H}$ NOESY spectra of C4YI13WFC in aqueous media showing NOE contacts. (A) NOEs corresponding to $\mathrm{C} 4$-acyl chain (highlighted in blue) to $\mathrm{Y} 1$ and aromatic aminoacids assigned. (B) long range side chain-side chain NOEs between W4 and F12. (C) NOEs corresponding to indole ring of W4 and F12 beta protons.

Figure 3.1-11, A summarizes all ranges of NOEs that are present between the residues in C4YI13WFC. Medium range NOEs of $\mathrm{C} \alpha \mathrm{H} / \mathrm{HN}$ of $i$ to $i+2$ type were noted between residues R8, K9, F10, C11, F12 and I13 with long range NOEs between W4 and F12 (Figure 3.1-11B). These NOEs revealed that the peptide folds into amphipathic structure stabilized by the disulfide bond. Because the $\mathrm{C} 11$ residue is dimerized with the adjacent unit $\mathrm{C} 11$ residue, the peptide folds in such a way that the aromatic lock is formed with all the basic residues facing one side. Furthermore, this 
NOE interaction between the W4 and F12 seems to be stabilized by the acyl chain present in the N-terminus. C4-acyl chain showed NOE only to Y1 indicating absence of long range packing of acyl chain with other residues.

(A)

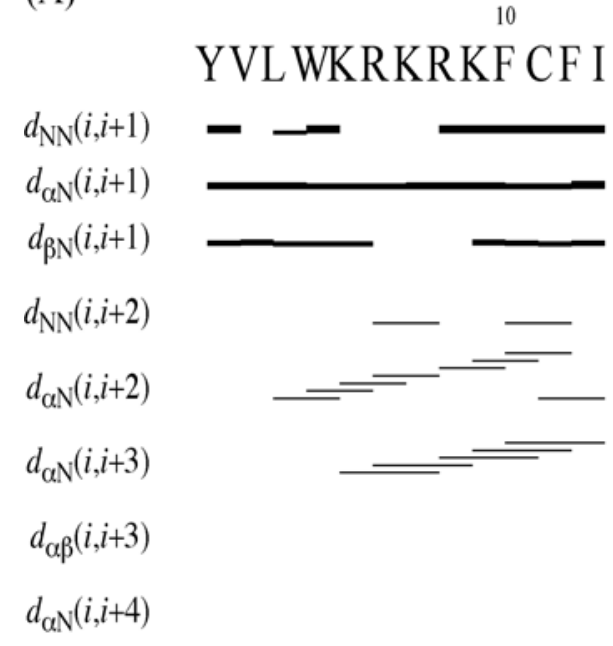

(B)

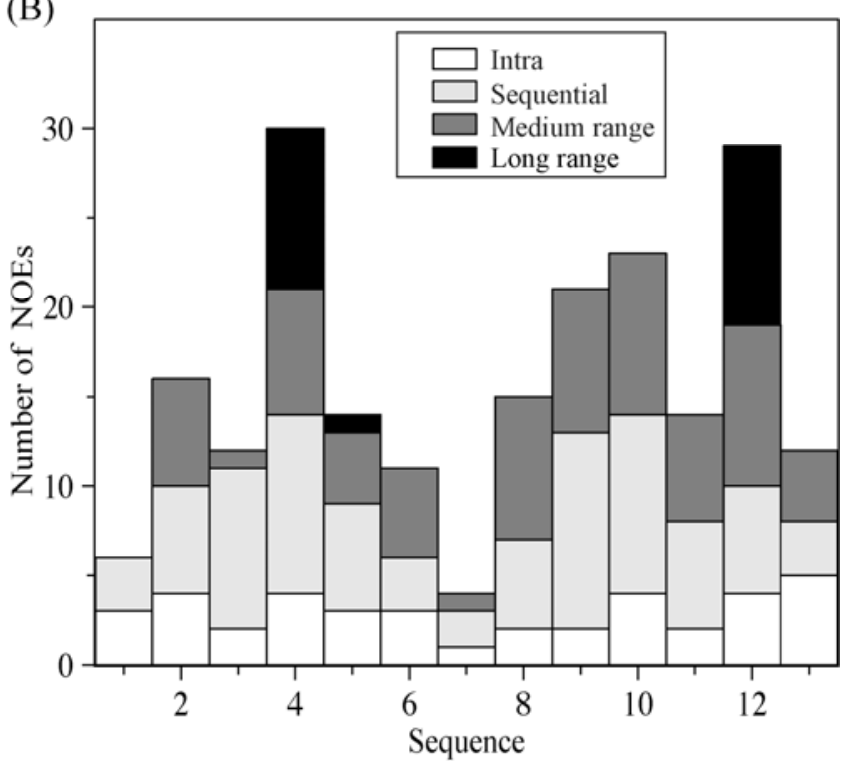

Figure 3.1-11: Bar diagram summarizing NOEs observed for C4YI13WFC peptide (monomer) in aqueous solution.

To further validate the importance of dimerization and N-terminal acylation, two dimensional ${ }^{1} \mathrm{H}-{ }^{1} \mathrm{H}$ TOCSY and NOESY spectra were obtained for C4YI13WF, acylated isoleucine analog and YI13WFC, nonacylated cysteine analog.

As can be seen in Figure 3.1-12B, the nonacylated cysteine analog, YI13WFC shows very weak NOE contacts between W4 and F12 side chains. The NOEs corresponding to F12 beta and W4H4 protons were absent. Further the indole ring protons of W4 also found to exhibit relatively weak NOE contact with F12 beta protons. But all of these side chain interactions were found to be missing in C4YI13WF spectra (Figure 3.1-12, C). The NOESY spectra of C4YI13WF were characterized by the presence of weak intraresidue and sequential NOEs. The absence of NOE cross peaks between the aromatic side chain-side chain protons clearly indicated that the peptide is unstructured in aqueous solution. These observations illustrate that the cysteine residue which forms disulfide bridge with the adjacent unit folds the peptide in to an amphipathic structure. 
The advantage of the N-terminal acylation is also achieved through these experiments. Eventhough YI13WFC peptide is folded into amphipathic structure, the intensity of the cross peaks arising from interaction between W4 and F12 was very weak when compared to its acylated analog. So the dimerization helps the peptide to become folded with characteristic aromatic lock and the acylation further helps to stabilize this amphipathic fold. Further structure calculations were carried out for C4YI13WFC peptide.

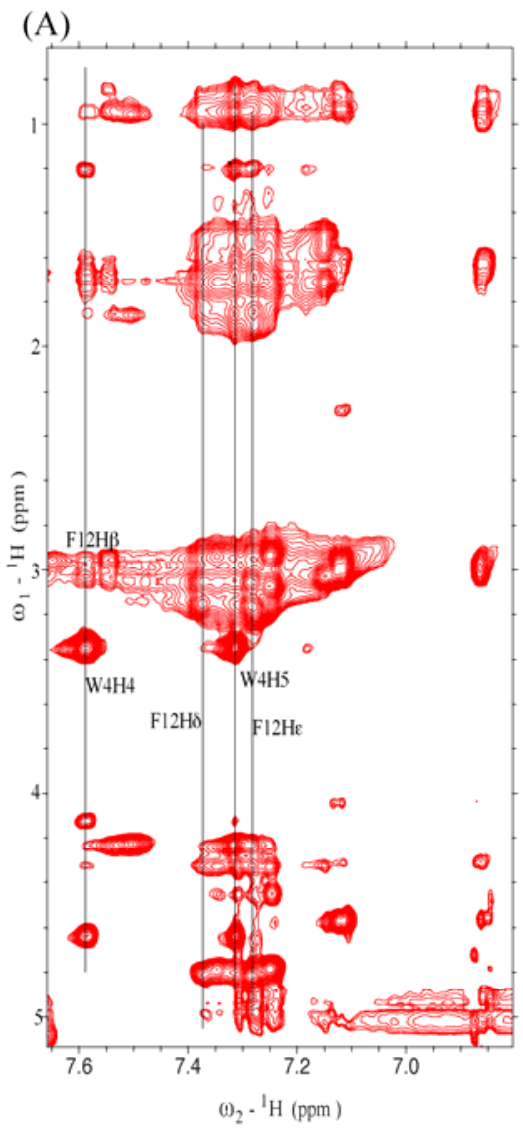

(B)

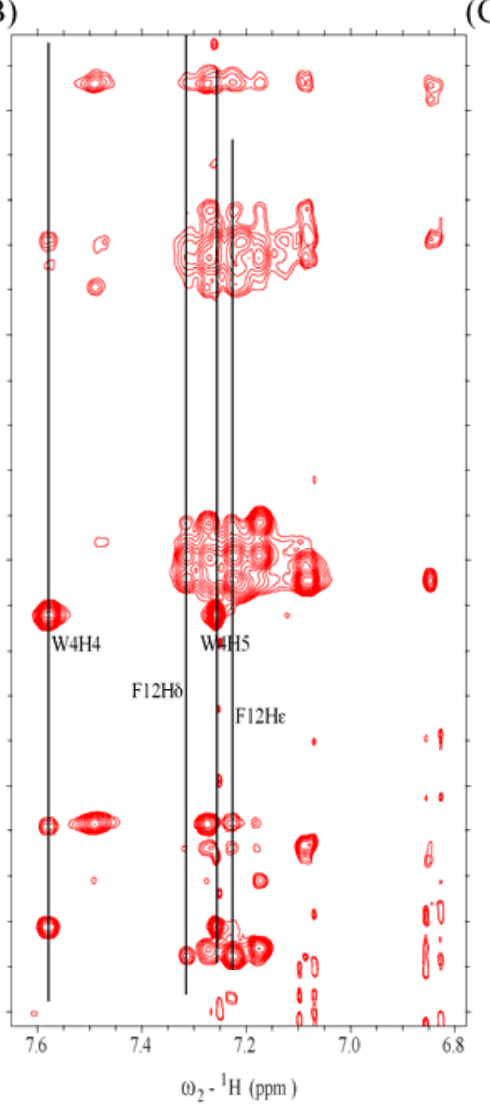

(C)

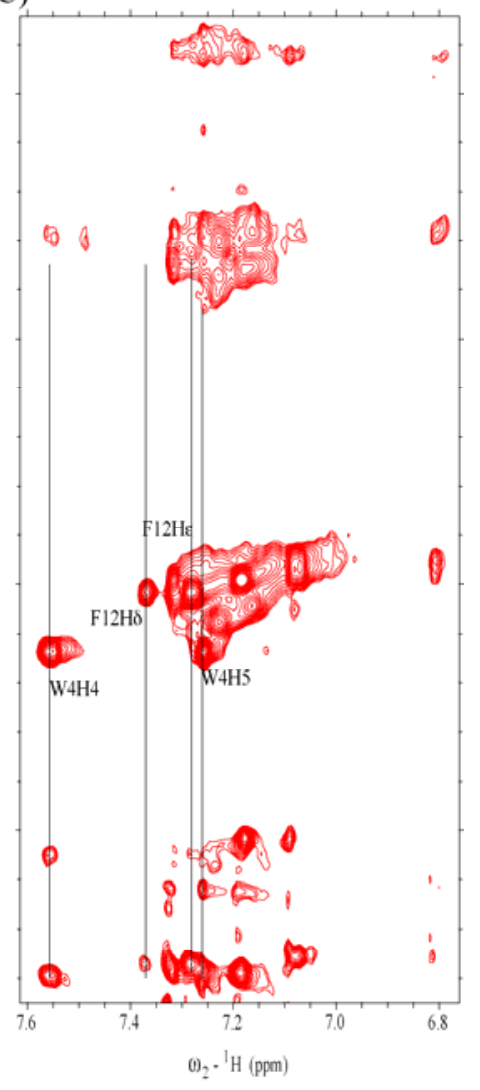

Figure 3.1-12: Selected regions of two-dimensional ${ }^{1} \mathrm{H}^{-1} \mathrm{H}$ NOESY spectra of (A) C4YI13WFC, (B) YI13WFC and (C) C8YI13WF correlating aromatic side chain-side chain interactions between W4 and F12.

\subsubsection{Structure of C4YI13WFC in aqueous state:}

Structure determination of C4YI13WFC was initiated by calculating for monomer first followed by joining two monomers using glycine linkers. The structure for a dimeric unit was obtained using 207 distance restraints (Table 3.1-10). 
Table 3.1-10: Summary of structural statistics of 20 lowest energy structures of dimeric C4YI13WFC in aqueous state.

\begin{tabular}{|c|c|}
\hline & C4YI13WFO \\
\hline $\begin{array}{l}\text { Distance restraints } \\
\text { intraresidue }(|i-j|=0) \\
\text { sequential } \quad(|i-j|=1) \\
\text { medium range }(2 \leq|i-j| \leq 4) \\
\text { long range }(|i-j| \leq 5) \\
\text { total NOE constraints }\end{array}$ & $\begin{array}{l}22 \\
110 \\
48 \\
26 \\
207\end{array}$ \\
\hline $\begin{array}{l}\text { Deviation from mean structure } \\
\text { backbone atoms }\left(\mathrm{N}, \mathrm{C} \alpha, \mathrm{C}^{\prime}\right)(\AA) \\
\text { heavy atoms }(\AA)\end{array}$ & \begin{tabular}{|l|}
0.76 \\
1.6
\end{tabular} \\
\hline $\begin{array}{l}\text { Ramachandran plot for the mean } \\
\text { structure } \\
\% \text { residues in the most favourable and } \\
\text { additionally allowed regions }\end{array}$ & 100 \\
\hline $\begin{array}{l}\% \text { residues in the generously allowed } \\
\text { region } \\
\% \text { residues in the disallowed region. }\end{array}$ & $\begin{array}{l}0 \\
0\end{array}$ \\
\hline
\end{tabular}

After the removal of glycine linkers, the rmsd value for all the heavy atoms is confined to $1.6 \AA$ (Table 3.1-10). Structure validation was carried out using Ramachandran plot. All of the residues were found to exist in the most favorable and additionally allowed region. A representative structure of the C4YI13WFC peptide is shown in Figure 3.1-13, A, delineating side chain packing between W4 and F12 aromatic residues (highlighted in orange color) with the oxidized cysteine bonds in yellow color. The electrostatic surface figure generated using the structure calculation of the dimers revealed that all of the positive charges arising from polar amino acids 
are segregated to one side of the peptide with the other side occupied with hydrophobic aromatic packing of W4-F12 and C4 acyl chain anchored Y1 (Figure $3.1-13, \mathrm{~B})$.

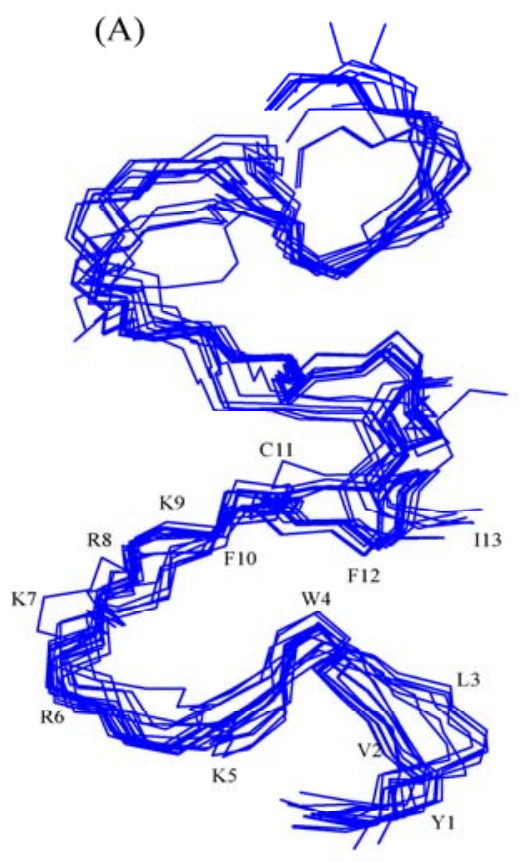

(B)

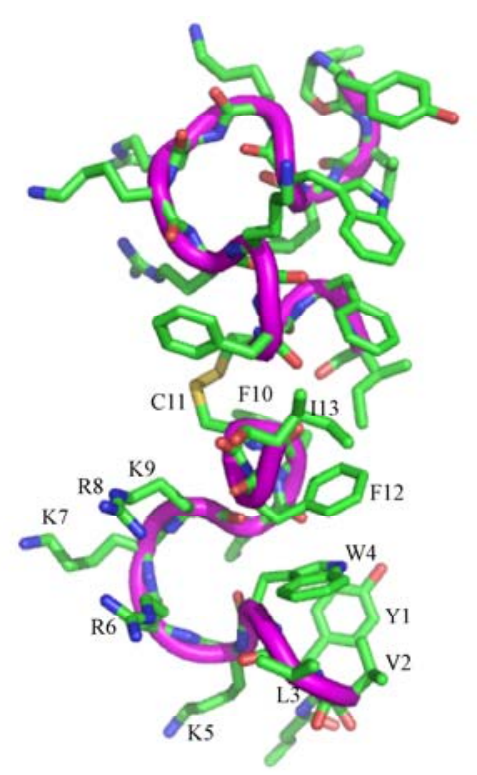

(C)

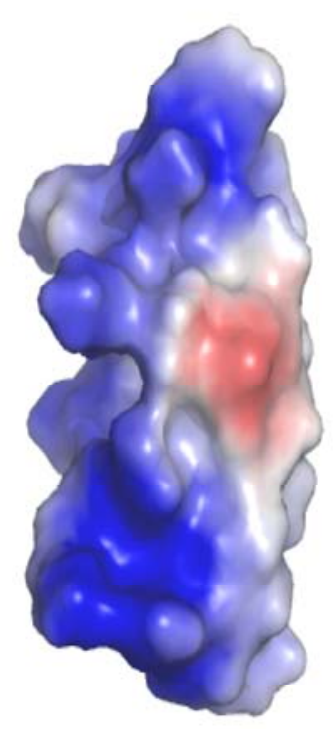

Figure 3.1-13: (A) Superimposition of backbone of 20 lowest energy structures of dimeric C4YI13WFC, (B) Single model representation of C4YI13WFC dimer in aqueous media and (C) electrostatic surface representation of dimers, blue cationic residues, red- hydrophobic residues.

\subsubsection{Structure of C4YI13WFC in LPS:}

A solution containing C4YI13WFC peptide was titrated with various concentrations of LPS. There was little NMR line broadening effect observed for the peptide, a phenomena characeristic of tight binding complexes. In the presence of LPS, C4YI13WFC peptide showed very similar NOE connectivity as observed for free peptide. Interestingly, NOEs were detected between the sugar moieties of lipid A with F12 residue of C4YI13WFC peptide (Figure 3.1-14). This observation confirmed the binding of designed peptide with the lipidA moiety of LPS. 
When acylated isoleucine analog was titrated with LPS, no peak broadening was observed even at concentration as high as $200 \mu \mathrm{M}$. It should be recalled that in spite of higher hydrophobicity, peptide lacks antibacterial activity and $\mathrm{K}_{\mathrm{d}}$ value of this peptide for LPS binding is much higher when compared to its cysteine analog. The possible explanation could be that the folding of the C4YI13WF peptide is hindered by the presence of extra lysine residue. In the case of C4YI13WFC, the disulfide bridge facilitates the folding of the peptide with aromatic lock and it can thus easily accommodate the extra lysine residue in the amphipathic boomerang structure.

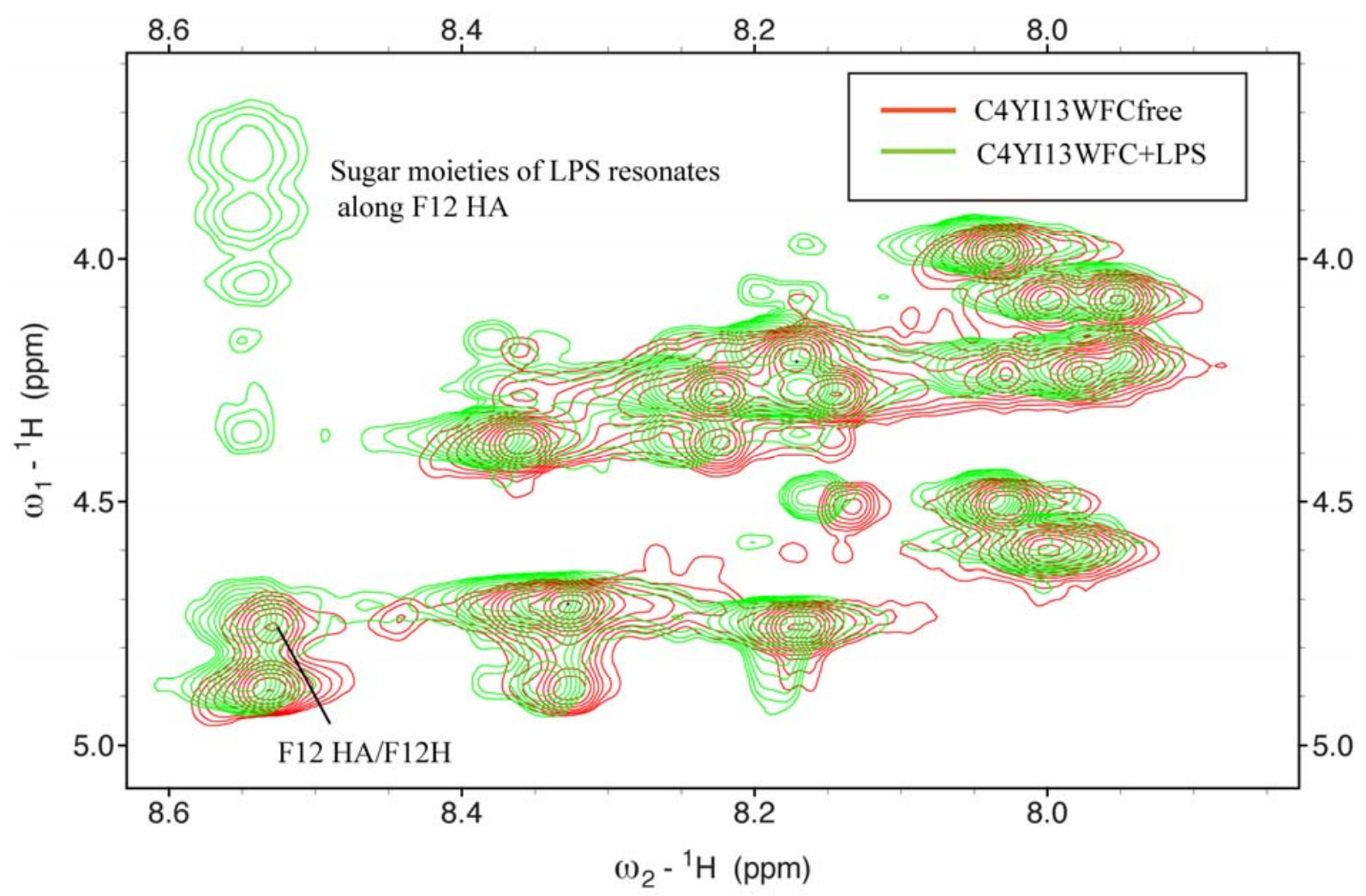

Figure 3.1-14: Two dimensional ${ }^{1} \mathrm{H}-{ }^{1} \mathrm{H}$ spectra of C4YI13WFC free (red) and C4YI13WFC in the presence of LPS (green) showing signals from sugar moieties of LPS resonating along Phe 12.

\subsubsection{Discussion:}

The chemical composition of bacterial membranes and mammalian membranes are different. The outer membrane of Gram negative bacteria is coated with LPS which is responsible for endotoxic property of the bacteria. LPS is also responsible for 
structural integrity of the membrane. LPS is found to be the primary responsible agent for sepsis and hence development of agents that neutralize LPS can be the therapeutic target for antisepsis agents.

Antimicrobial peptides exhibit a wide variety of structural motifs. Inspite of this structural diversity, AMPs are characterized by its amphipathic structure that is needed to bind to bacterial membrane. Beta sheet AMPs exist either in the cyclic form or oligomers to compensate for the loss of entropy. The beta hairpin is generally formed by disulfide bridges as in case of Tachyplesins, Protegrins and Lactoferricin [35]. Interestingly the disulfide bonded beta sheet AMPs are structured in aqueous media and this structure acts as intermediate state in membrane translocation of the AMPs [158].

In an attempt to design such anti-microbial agents with anti-endotoxic property, a library of peptides was designed and its activity chart was improved by structure based rational design. The first generation peptide YW12 (Y1-V2-L3-W4-K5-R6-K7-R9M9-I10-F11-I12) was designed based on the LPS recognition site of FhuA complex. The peptide folds into amphipathic structure in complex with LPS. The centrally placed K5-R6-K7-R8 cationic residues face one side of the boomerang shape with the long range W4-M9 facing other side. Hence in the second generation, peptides were designed with aromatic acids replacing M9. In this generation the peptide acquired amphipathic structure with aromatic lock between Trp4 and X9 (X is Phe or Trp or Tyr) in the presence of LPS. It was also demonstrated that the long range aromatic packing between $\mathrm{W} 4$ and $\mathrm{X} 9$ is important for antiendotoxic and antibacterial activities of the peptide [150]. This aromatic packing has also imparted a specific orientation to the hydrophobic termini resembling a boomerang. If the residues 4 and 9 are replaced with alanine and leucine, the antimicrobial and antiendotoxic activity is lost. This explained the importance of specific aromatic packing in structure and activity. It was established that aromatic-aromatic interactions make higher contribution to beta hairpin stability than aliphatic-aromatic interactions [159].

The third generation of peptides was designed with the following modifications:

* Addition of different length acyl chain to the N-terminus increasing the hydrophobicity of the peptide and thereby improving the antibacterial activity. 
* Introduction of one more lysine residue to improve the solubility of the peptides and

* Replacement of Ile11 with Cys to form a disulfide bridge and in turn form dimers.

N-terminal acylation of YI13WF and YI13WFC improved antibacterial activity as revealed by antimicrobial assays and antiendotoxic activity as revealed by LAL assay. The dimerization has helped the peptide to improve antibacterial activity. The isoleucine analogs were inactive at concentrations as high as $200 \mu \mathrm{M}$ whereas the cysteine analogs can inhibit bacterial growth as low as $3 \mu \mathrm{M}$. The cysteine analogs have higher hydrophobicity when compared to the isoleucine analogs and better killing activity as well. However the retention time as measured with HPLC, of the largely inactive peptide C8YI13AAC is higher than the active C4YI13WFC because of the presence of two alanine residues and the $\mathrm{C} 8$ chain. But the MIC values are much higher when compared to C4YI13WFC. Also, C8YI13AAC peptide does not interact with LPS as shown with ITC and DLS results. This clearly indicates that hydrophobicity measure alone cannot contribute to the best design of antimicrobial peptide but also there is need for more characteristic interactions.

$\mathrm{N}$-terminal acylation is shown to improve antibacterial activity because of increase in hydrophobicity of the peptides. But this improvement depends on chain length. An increase in hydrophobicity above certain threshold level diminishes cell selectivity [160]. The acylated disulfide bonded peptide certainly shows better antibacterial activity when compared to isoleucine analogs. When the cysteine analogs were tested for antiendotoxic activity by LAL assay, the acylated peptides show $\sim 80 \%$ neutralization at $1.0 \mathrm{EU} / \mathrm{ml}$ LPS concentration. Zeta potential measurements also support that the acylated cysteine analogs have better ability of neutralizing LPS when compared to all of the analogs.

The localization of these peptides in LPS was analyzed by biophysical methods such as fluorescence, ITC and DLS. The acylated dimerized C4YI13WFC peptide dissociates LPS micelles effectively and its tight binding with LPS as revealed by $\mathrm{K}_{\mathrm{d}}$ value of $0.45 \mu \mathrm{M}$ suggest that this peptide can be considered for further development for antisepsis agents. Overall biophysical studies suggest that for beta boomerang 
analogs, like Mastoparan-X acylated analogs [125], there is balance for peptide hydrophobicity. An increased hydrophobicity can impair the peptide selectivity towards negatively charged membrane and mammalian membrane.

These activity results were correlated with high resolution structure determined by NMR spectroscopy. The structure calculations suggest that the cysteine analogs fold into beta sheet structure with aromatic lock characteristic of YI12 peptides in aqueous media. But in the case of YI13WFC and C4YI13WFC, the characteristic aromatic interaction was found between W4 and F12 i.e. the aromatic residue placed after disulfide bridge. Hence the disulfide bridge folds the peptide in such a way that the side chains of W4 and F12 interact with each other with all of the cationic residues on the opposite side. Upon interaction with the membrane, the cationic residues compete with divalent cations (that hold LPS molecules together) further exerting ionic interactions with anionic phosphate groups in LPS. This brings about the dissociation of LPS micelles whereby disrupting membrane integrity of bacterial cells. This type of interaction was not observed in isoleucine analog.

In summary, the acylation and dimerization improves antibacterial and antiendotoxic activities of the designed peptides. However, the longer the acyl chain length, the more was the loss in membrane selectivity. Hence the C4YI13WFC peptide can be considered for further improvement as a result of its low toxicity and high LPS neutralization potency. 


\section{2 $\beta$-BOOMERANG FOLD TO CONQUER LPS TRAP}

\subsubsection{Peptide design:}

Lipopolysaccharides (LPS) present in the outer layer of outer membrane of Gram negative bacteria acts as impermeable barrier for many hydrophobic compounds including antibiotics and antimicrobial peptides [62]. Some antimicrobial peptides, ribosomally synthesized or denovo designed, aggregate in the presence of LPS, which once bypasses the LPS layer becomes active. For example, peptides like Temporin-1b, Temporin-1a which are naturally available as one of the host defense molecules on frog skin are found to undergo oligomerisation on LPS [108], [161]. In synergy with Temporin-11, these peptides become active [108]. A library of denovo designed peptides containing Leu and Lys also found to aggregate on LPS layer whose diastereomeric analog that did not aggregate, was found to be active [162].

A structured LPS binding motif (W-K-R-K-R-F) was identified from a library of designed YI12 (Y-V-L-W-K-R-K-R-F-I-F-I) peptides. Upon interaction with LPS micelles, the aromatic packing of W4 and F9 along with the positive charges K5-R6K7-R8 imparted a boomerang shape to the peptide and the hydrophobicity generated by the neighbouring aminoacids established the antimicrobial and antiendotoxic activity. When W4 and F9 were mutated to alanine, the activity was lost [150]. A short analog with only the motif accompanied by glycine at both termini was also synthesized. This short peptide GG8 also found to fold into characteristic boomerang shape upon contact with LPS micelles with W2 and F7 conserved packing. But since the hydrophobicity needed to disrupt the cells were not attained with glycine truncation, the peptide remained largely inactive [150]. But these studies have led to the identification of Structured LPS binding motif W-K-R-K-R-F.

The hybrid peptides were designed by conjugating the peptides that are trapped on LPS layer in N-terminal and GG8 in C-terminal. Temporin-1b, Temporin-1a and K5L7 peptides were selected for conjugation with GG8 giving rise to LG21, FG21 and KG20 respectively (Table 3.2-1). One of the shortest antimicrobial peptide Temporin-SHf (FF8), rich in Phe is inactive because of its short chain length [163] 
and N-terminal part of LL37 (L1-K12) is inactive because of less hydrophobicity [164]. These two peptides were also conjugated with GG8 forming FG16 and LG20 (Table 3.2-1).

Table 3.2-1: Primary structure of designed peptides, alanine and truncated mutants of LG21.

\begin{tabular}{|l|l|}
\hline Peptide & Sequence \\
\hline LG21 & L-L-P-I-V-G-N-L-L-K-S-L-L-G-W-K-R-K-R-F-G \\
\hline FG21 & F-L-P-L-I-G-R-V-L-S-G-I-L-G-W-K-R-K-R-F-G \\
\hline KG20 & K-L-L-L-K-L-K-L-K-L-L-K-G-W-K-R-K-R-F-G \\
\hline FG16 & F-F-F-L-S-R-I-F-G-W-K-R-K-R-F-G \\
\hline LG21 K10A & L-L-P-I-V-G-N-L-L-A-S-L-L-G-W-K-R-K-R-F-G \\
\hline LG21 S11A & L-L-P-I-V-G-N-L-L-K-A-L-L-G-W-K-R-K-R-F-G \\
\hline LG21 K16A & L-L-P-I-V-G-N-L-L-K-S-L-L-G-W-A-R-K-R-F-G \\
\hline LG21 R17A & L-L-P-I-V-G-N-L-L-K-S-L-L-G-W-K-A-K-R-F-G \\
\hline LG21 K18A & L-L-P-I-V-G-N-L-L-K-S-L-L-G-W-K-R-A-R-F-G \\
\hline LG21 R19A & L-L-P-I-V-G-N-L-L-K-S-L-L-G-W-K-R-K-A-F-G \\
\hline LG14 & L-L-K-S-L-L-G-W-K-R-K-R-F-G \\
\hline LG15 & L-L-P-I-V-G-N-G-W-K-R-K-R-F-G \\
\hline LG21W15AF20A & L-L-P-I-V-G-N-L-L-K-S-L-L-G-A-K-R-K-R-A-G \\
\hline
\end{tabular}

Based on the results obtained in the extensive characterization of these hybrid peptides, LG21 peptide was selected for complete alanine scanning and truncation 
studies. Alanine scanning of the peptides help to define the role of each aminoacid in membrane permeability and antimicrobial activity [165]. The polar residues N7, S11 and the positively charged residues in LG21 were replaced with alanine in a series of analogs. N-terminal truncation of temporin-1b part of LG21 encompassing residues L1-N7, G14-G21 and C-terminal truncation of temporin-1b part of LG21 encompassing residues L8-G21 were also designed to ascertain the importance of leucine residues in temporin-1b (Table 3.2-1).

\subsubsection{Activity assays:}

\subsubsection{Minimum inhibitory concentration determination:}

The hybrid peptides, alanine and truncated mutants of LG21 were examined for antibacterial activity against 4 Gram negative and 4 Gram positive bacterial strains The MIC determination study was carried out in salt free MH broth and also in $100 \mathrm{mM}$ salt containing LB media.

\subsubsection{MIC determination of hybrid peptides:}

MIC assays for hybrid peptides LG21, FG21 and KG20 against Gram negative bacteria clearly demonstrated the ability of the peptides to kill bacterial cells even in rich LB medium.

The peptides Temporin 1 $\mathrm{Tb}$ and 1Ta that aggregates on LPS layer have 50-100 $\mu \mathrm{M}$ MIC values (Table 3.2-3). But after conjugation with GG8, hybrid peptides LG21 and FG21, demonstrated high antibacterial activity with MIC of $2 \mu \mathrm{M}$ in MH broth and 8,10 $\mu \mathrm{M}$ in LB media respectively (Table 3.2-2). 12-mer amphipathic alpha helical K5L7 peptide after conjugation with GG8 (KG20) also exhibited high antibacterial activity, but only in salt free MH broth (Table 3.2-2). In LB media, even though the MIC values were as low as $50-100 \mu \mathrm{M}$, it showed improvement when compared to parent peptide K5L7 [162]. Whereas temporin SHf and N-terminal LL37, even after conjugation with GG8 (FG16, LG20) does not demonstrate higher killing activity. But when compared against parent peptides, the hybrids definitely showed improvement in killing activity (Table 3.2-2). 
Table 3.2-2: Minimum inhibitory concentration (in $\mu \mathrm{M}$ ) determination of hybrid peptides in Gram negative bacteria.

\begin{tabular}{|l|l|l|l|l|l|l|l|l|l|l|}
\hline & \multicolumn{2}{l}{ LG21 } & \multicolumn{2}{l}{ FG21 } & \multicolumn{2}{l|}{ KG20 } & \multicolumn{2}{l|}{ FG16 } & \multicolumn{2}{l|}{ LG20 } \\
\cline { 2 - 11 } & MHB & LB & MHB & LB & MHB & LB & MHB & LB & MHB & LB \\
\hline $\begin{array}{l}\text { E.coli } \\
\text { (lab strain) }\end{array}$ & 2 & 8 & 2 & 10 & 4 & 100 & 10 & 25 & 50 & 25 \\
\hline $\begin{array}{l}\text { P.aeruginosa } \\
\text { ATCC 27853 }\end{array}$ & 4 & 8 & 2 & 8 & 4 & 50 & 50 & 25 & 50 & 50 \\
\hline $\begin{array}{l}\text { K.pneumoniae } \\
\text { ATCC 13883 }\end{array}$ & 3 & 8 & 4 & 8 & 10 & 100 & 50 & 100 & 50 & 100 \\
\hline $\begin{array}{l}\text { S.enterica } \\
\text { ATCC 14028 }\end{array}$ & 3 & 8 & 5 & 10 & 10 & 200 & 50 & 50 & 50 & 25 \\
\hline
\end{tabular}

Table 3.2-3: Comparison of MIC (in $\mu M$ ) values for hybrids with Temporins in Gram negative bacteria.

\begin{tabular}{|l|l|l|l|l|}
\hline Bacteria & LG21 & Temporin 1b & FG21 & Temporin 1a \\
\hline $\begin{array}{l}\text { E.coli } \\
\text { (lab strain) }\end{array}$ & 2 & 100 & 2 & 100 \\
\hline $\begin{array}{l}\text { P.aeruginosa } \\
\text { ATCC 27853 }\end{array}$ & 4 & 100 & 2 & 100 \\
\hline $\begin{array}{l}\text { K.pneumoniae } \\
\text { ATCC 13883 }\end{array}$ & 3 & $>200$ & 4 & $>200$ \\
\hline $\begin{array}{l}\text { S.enterica } \\
\text { ATCC 14028 }\end{array}$ & 3 & $>200$ & 5 & $>200$ \\
\hline
\end{tabular}

MIC of hybrids against Gram positive bacteria also showed better activity when compared to parent peptides. LG21 and FG21, in particular showed very high activity in the range of $0.5-2 \mu \mathrm{M}$ in $\mathrm{MH}$ media (Table 3.2-4). KG20 also exhibited improved 
sterilization of Gram positive bacteria but only in salt free MH broth. The hybrids FG16 and KG20 that had low killing activity in Gram negative bacteria (Table 3.2-2) show only little improvement with Gram positive bacteria (Table 3.2-4).

Table 3.2-4: Minimum inhibitory concentration (in $\mu \mathrm{M}$ ) of GG8 hybrids against Gram positive bacteria.

\begin{tabular}{|c|c|c|c|c|c|c|c|c|c|c|}
\hline \multirow{2}{*}{$\begin{array}{l}\text { Gram } \\
\text { positive } \\
\text { bacteria }\end{array}$} & \multicolumn{2}{|l|}{ LG21 } & \multicolumn{2}{|l|}{ FG21 } & \multicolumn{2}{|l|}{ KG20 } & \multicolumn{2}{|l|}{ FG16 } & \multicolumn{2}{|l|}{ LG20 } \\
\hline & MHB & LB & MHB & LB & MHB & LB & MHB & LB & MHB & LB \\
\hline $\begin{array}{l}\text { B.subtilis } \\
\text { (lab strain) }\end{array}$ & 2 & 10 & 1 & 10 & 4 & 50 & 4 & 12.5 & 30 & 25 \\
\hline $\begin{array}{l}\text { S.aureus } \\
\text { ATCC } \\
25923\end{array}$ & 2 & 12.5 & 0.5 & 10 & 4 & 25 & 10 & 25 & 30 & 12.5 \\
\hline $\begin{array}{l}\text { S.pyogenes } \\
\text { ATCC } \\
19615\end{array}$ & 2 & 8 & 3 & 12.5 & 10 & 100 & 30 & 25 & 50 & 12.5 \\
\hline $\begin{array}{l}\text { E.faecalis } \\
\text { ATCC } \\
29212\end{array}$ & 5 & 12.5 & 10 & 10 & 10 & 50 & 30 & 12.5 & 50 & 25 \\
\hline
\end{tabular}

Taken together, the MIC determination suggests that the conjugations of GG8 with the peptides are very much selective for those peptides that are aggregating on the LPS layer. Parent peptides of FG16 and LG20 are inactive because of short sequence length and less hydrophobicity respectively $[163,164]$. Even after conjugation with LPS binding motif the threshold hydrophobicity needed to disrupt the bacterial cells may not have been achieved that results in low antibacterial activity.

\subsubsection{Determination of MIC for alanine and truncated mutants of LG21:}

As can be seen from Table 3.2-5, the MIC of truncated mutants clearly dissect the importance of C-terminal leucine residues of Temporin-1b (LG14) in bringing about 
the activity whereas N-terminal truncated LG15 remains largely inactive against both Gram negative and Gram positive bacteria (Table 3.2-5).

Table 3.2-5: MIC values (in $\mu \mathrm{M}$ ) of truncated LG21 mutants in Gram negative and Gram positive bacteria.

\begin{tabular}{|l|l|l|}
\hline & LG14 & LG15 \\
\hline E.coli (lab strain) & 4 & 50 \\
\hline P.aeruginosa (ATCC 27853) & 4 & 100 \\
\hline K.pneumoniae (ATCC 13883) & 50 & 200 \\
\hline S.enterica (ATCC 14028) & 10 & 200 \\
\hline B.subtilis. (lab strain) & 8 & 200 \\
\hline S.aureus (ATCC 25923) & 10 & 200 \\
\hline S.pyogenes (ATCC 19615) & 10 & 200 \\
\hline E.faecalis (ATCC 29212) & 10 & 200 \\
\hline
\end{tabular}

Table 3.2-6: MIC values (in $\mu \mathrm{M}$ ) of alanine mutants of LG21 in Gram negative bacteria. The values of LG21 are also included for comparison.

\begin{tabular}{|l|l|l|l|l|}
\hline & $\begin{array}{l}\text { E.coli } \\
\text { (lab strain) }\end{array}$ & $\begin{array}{l}\text { P.aeruginosa } \\
\text { ATCC 27853 }\end{array}$ & $\begin{array}{l}\text { K.pneumoniae } \\
\text { ATCC 13883 }\end{array}$ & $\begin{array}{l}\text { S.enterica } \\
\text { ATCC 14028 }\end{array}$ \\
\hline LG21 & 2 & 4 & 3 & 3 \\
\hline LG21N7A & 4 & 4 & 4 & 4 \\
\hline LG21K10A & 8 & 8 & 4 & 8 \\
\hline LG21S11A & 8 & 8 & 8 & 8 \\
\hline LG21K16A & 10 & 12.5 & 12.5 & 25 \\
\hline LG21R17A & 100 & 100 & 200 & $>200$ \\
\hline LG21K18A & 25 & 25 & 50 & 100 \\
\hline LG21R19A & 100 & 200 & 200 & $>200$ \\
\hline LG21W15AF20A & 100 & 100 & 200 & $>200$ \\
\hline
\end{tabular}


Table 3.2-7: MIC values (in $\mu \mathrm{M}$ ) of alanine mutants of LG21 in Gram positive bacteria. The values of LG21 are also included for reference.

\begin{tabular}{|l|l|l|l|l|}
\hline & $\begin{array}{l}\text { B.subtilis } \\
\text { (lab strain) }\end{array}$ & $\begin{array}{l}\text { S.aureus } \\
\text { ATCC 25923 }\end{array}$ & $\begin{array}{l}\text { S.pyogenes } \\
\text { ATCC 19615 }\end{array}$ & $\begin{array}{l}\text { E.faecalis } \\
\text { ATCC 29212 }\end{array}$ \\
\hline LG21 & 2 & 2 & 2 & 5 \\
\hline LG21N7A & 4 & 8 & 10 & 10 \\
\hline LG21K10A & 8 & 4 & 4 & 4 \\
\hline LG21S11A & 4 & 2 & 1 & 1 \\
\hline LG21K16A & 25 & 25 & 50 & 50 \\
\hline LG21R17A & 50 & 50 & 50 & 100 \\
\hline LG21K18A & 25 & 50 & 50 & 100 \\
\hline LG21R19A & 50 & 50 & 100 & 100 \\
\hline LG21W15AF20A & 100 & 100 & 200 & $>200$ \\
\hline
\end{tabular}

MIC was also determined for alanine mutants of LG21. The mutants activity were in the range of $\mathrm{N} 7 \mathrm{~A}>\mathrm{K} 10 \mathrm{~A}>\mathrm{S} 11 \mathrm{~A}>\mathrm{K} 16 \mathrm{~A}>\mathrm{K} 18 \mathrm{~A}>\mathrm{R} 17 \mathrm{~A}>\mathrm{R} 19 \mathrm{~A}>\mathrm{W} 15 \mathrm{AF} 20 \mathrm{~A}$ (Table 3.2-6, Table 3.2-7). The replacement of N7, K10 and S11 with Ala does not seem to have much effect on killing activity with MIC values of 3-8 $\mu \mathrm{M}$. But mutation in the cationic residues of GG8 seems to affect the activity of LG21. Even though K16 is not affected, the mutation of R17, K18 and R19 are affected drastically (Table 3.2-6). In Gram negative bacteria, R17 and R19 mutants exhibit no activity at all (Table 3.2-6). When aromatic aminoacids W15 and F20 are replaced with Ala, it remained largely inactive. MIC determination of alanine mutants certainly demonstrate that the cationic residues in $\beta$-boomerang motif are important in bringing about the killing activity when compared to polar and cationic residues in Temporin-1b.

Considering the MIC results of all the peptides, further extensive SAR (StructureActivity Relationship) studies were carried out for active hybrid peptides LG21, FG21, KG20 and LG21R19A alanine mutant. 


\subsubsection{Hemolytic activity:}

Temporins were non hemolytic whereas K5L7 was $80 \%$ hemolytic at $100 \mu \mathrm{M}$ peptide concentration $[161,162]$. In order to check the ability of the conjugated peptides to lyse red blood cells, the peptides were analyzed for hemolysis along with Temporin$1 \mathrm{~b}$ as control. The chimerisation of LPS binding motif to aggregating peptides has conserved the non-hemolytic property. In the case of $\mathrm{K} 5 \mathrm{~L} 7$, the conjugation has rendered non-hemolytic ability to the peptide that even at $100 \mu \mathrm{M}$, it is only $2.4 \%$ hemolytic (Table 3.2-8).

Table 3.2-8: Percentage of hemolysis of peptides at two different concentrations with Temporin-1b as control.

\begin{tabular}{|l|l|l|}
\hline Peptides & \% of Hemolysis at $50 \mu \mathrm{M}$ & \% of Hemolysis at $100 \mu \mathrm{M}$ \\
\hline LG21 & -1.1 & 8.5 \\
\hline FG21 & 0.52 & 9.9 \\
\hline KG20 & -8.5 & 2.4 \\
\hline LG21R19A & 16 & 25 \\
\hline Temporin-1b & -2.5 & 4.8 \\
\hline
\end{tabular}

In the case of temporins also, the conjugated hybrid peptides LG21 and FG21 show very less hemolytic activity as comparable with the parents (Table 3.2-8). The mutant LG21R19A peptide showed $25 \%$ of hemolytic activity at $100 \mu \mathrm{M}$ peptide concentration (Table 3.2-8). It should also be noted that the active hybrid peptides LG21, FG21 and KG20 exerted hemolytic activity in the peptide concentration that is several fold greater than its MIC value.

\subsubsection{LAL assay for LPS neutralization activity:}

The results of MIC and hemolytic assays clearly demonstrated the selective ability of the hybrid peptides to disrupt only bacterial cells (Table 3.2-2 - Table 3.2-8). In order to develop an antisepsis agent, the peptide should effectively neutralize LPS that is released as a result of bacterial killing. The ability of the hybrid peptides to neutralize LPS was assayed using LAL chromogenic kit as mentioned in Methods section. 
All of the active hybrid peptides tested show constant increase in LPS neutralization with increasing concentration of peptides. They show around $80 \%$ of neutralization ability at $10 \mu \mathrm{M}$ itself after which it reaches a plateau (Figure 3.2-1). Interestingly LG21 shows $50 \%$ neutralization in its MIC concentration of $3 \mu \mathrm{M}$ itself (Figure 3.2-1). On the other hand one, LG21R19A mutant show no or very poor LPS neutralization ability (Figure 3.2-1).

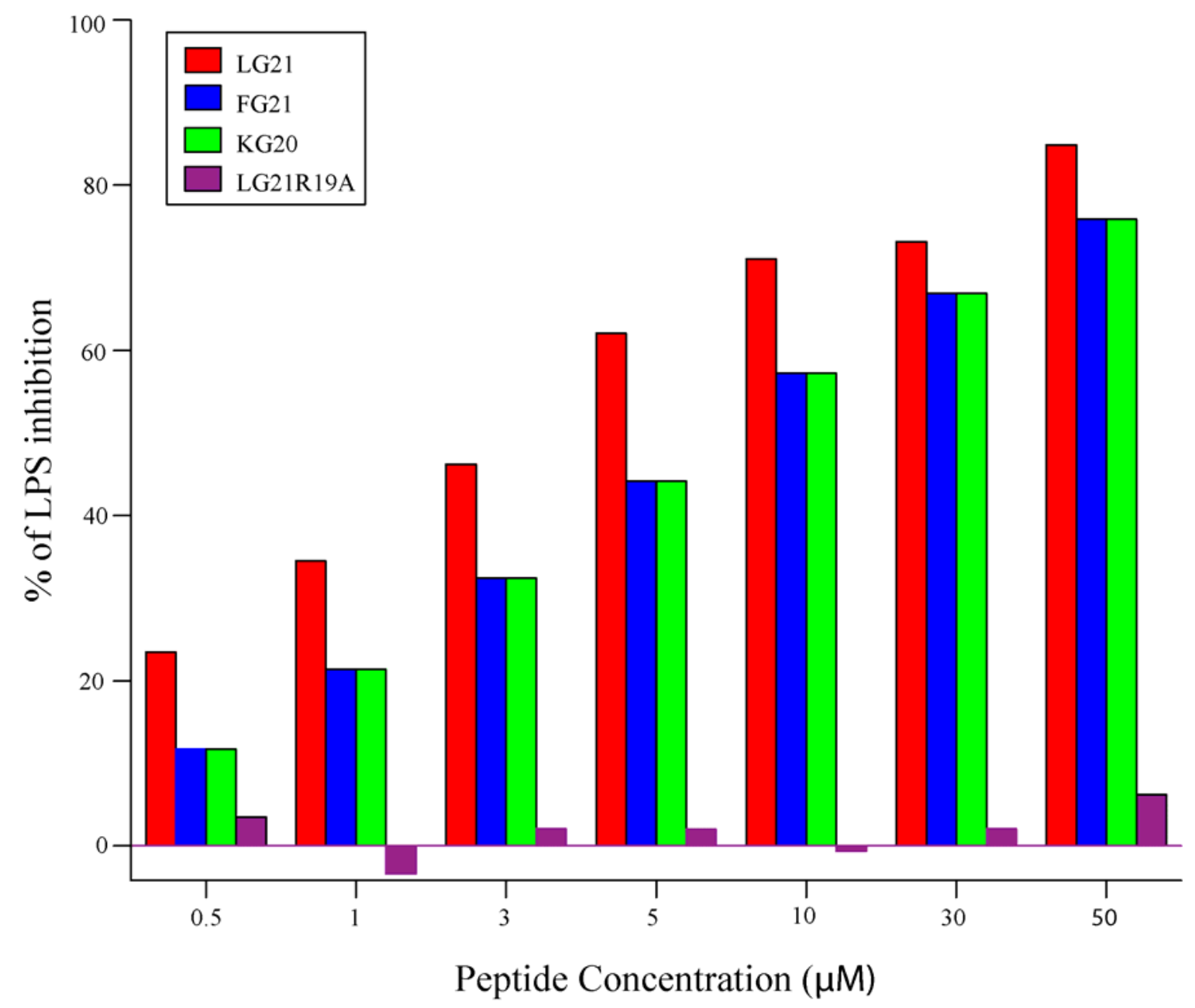

Figure 3.2-1: A bar diagram representing the LPS neutralization activity of designed peptides at 1.0 EU. 


\subsubsection{Biophysical characterization:}

\subsubsection{Rhodamine fluorescence dequenching:}

Unlike the other extrinsic dyes used in the characterization of activity of GG8 hybrids, Rhodamine is a fluorescent dye that cannot be used to judge the polarity of the environment. Instead, when the rhodamine molecules are in close proximity, they self associate and results in quenching of its fluorescence intensity. This property of rhodamine is exploited in studying the organizational behaviour of GG8 hybrids in the presence of LPS. The active peptides LG21, FG21 and KG20 were labeled with rhodamine at N-terminus. It is known that temporins $1 \mathrm{a}$ and $1 \mathrm{~b}$ undergo self association to form higher order oligomers which results in impermeability of those peptides in Gram negative bacteria[161].

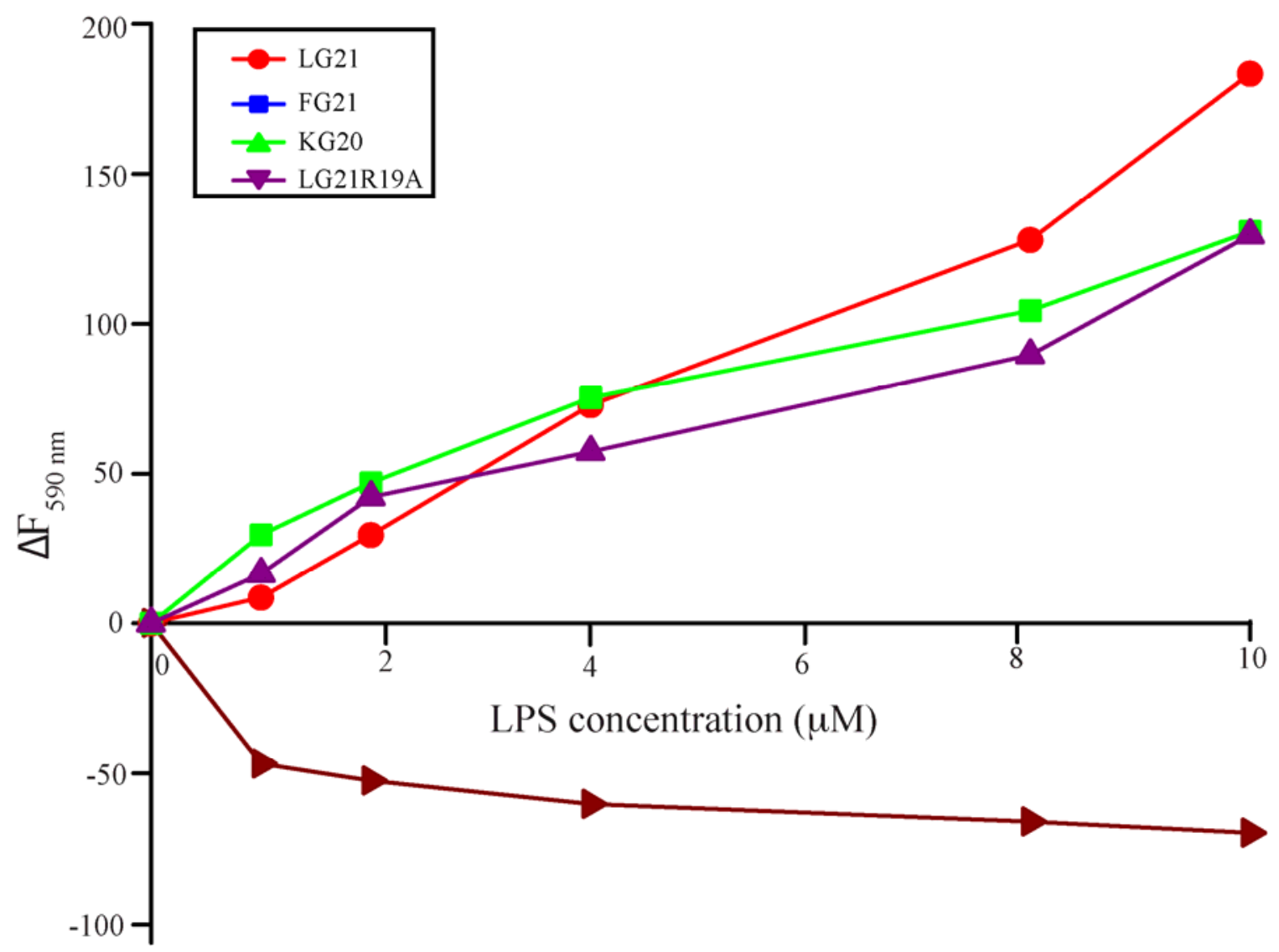


Figure 3.2-2: Rhodamine fluorescence dequenching by the hybrid peptides and Ala mutant of LG21. $\Delta \mathbf{F}_{590 \mathrm{~nm}}$ is the difference in fluorescence intensity after and before addition of various concentrations of LPS to rhodamine labeled LG21, FG21, KG20 and LG21R19A.

When rhodamine labeled GG8 hybrids, rhoLG21, rhoFG21 and rhoKG20 were added with increasing concentration of LPS, the fluorescence intensity show gradual increase in fluorescence intensity (Figure 3.2-2). This explains the organization of conjugated peptides in the presence of LPS. On the other hand, rhoLG21R19A showed gradual decrease in intensity signal as concentration of LPS increases (Figure 3.2-2). Peptide concentration of $3 \mu \mathrm{M}$ has been used for the titrations. This result clearly explains that the hybrid peptides LG21, FG21 and KG20 stay as monomers and hence can permeate through the LPS layer. Whereas LG21R19A undergoes self association, which brings the rhodamine monomers close enough that, it undergoes self quenching and hence exhibit negative changes.

\subsubsection{Permeabilization of outer membrane:}

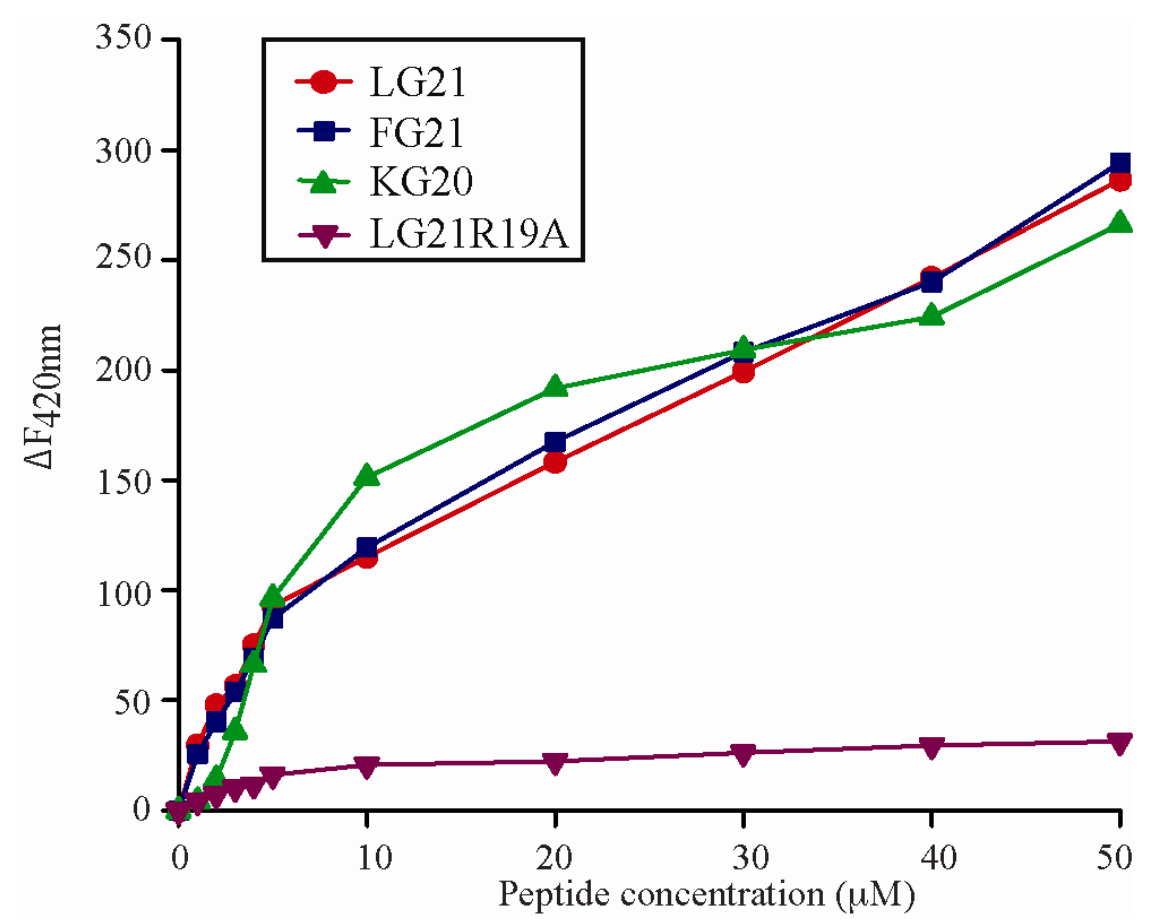


Figure 3.2-3: Outer membrane permeabilizing ability of hybrids and Ala mutant peptide in $E$.coli. $\Delta \mathbf{F}_{420 \mathrm{~nm}}$ is the difference between fluorescence intensities after and before addition of peptides at emission max of NPN at $420 \mathrm{~nm}$.

One of the biggest challenges faced by antimicrobial compounds is to disrupt the outer membrane of bacteria. The bacterial membranes are organized in such a way that it excludes the entry of hydrophobic molecules like extrinsic fluorophores. NPN (1-Nphenylnaphthylamine) is also one such dye, which is precluded from bacterial outer membrane.

The hybrid peptides LG21, FG21 and KG20 showed continuous increase in fluorescence intensity as the concentration of peptide increases (Figure 3.2-3). On the other hand, LG21R19A show negligible or no increase in fluorescence intensity at all (Figure 3.2-3). This result comes as evidence that the hybrid peptides can effectively disrupt the outer membrane, which permeates NPN dye into hydrophobic environment whereas LG21R19A cannot disrupt the outer membrane at all.

\subsubsection{Permeabilization of both outer and inner membranes:}

The next barrier probably for the antimicrobial peptides would be to disrupt inner membrane so that the peptides can bind to various intracellular targets inside the cell and kill them. SYTOX green is a positively charged dye that is again excluded from bacterial cells. This dye binds exclusively to nucleic acids and fluoresces strongly. 


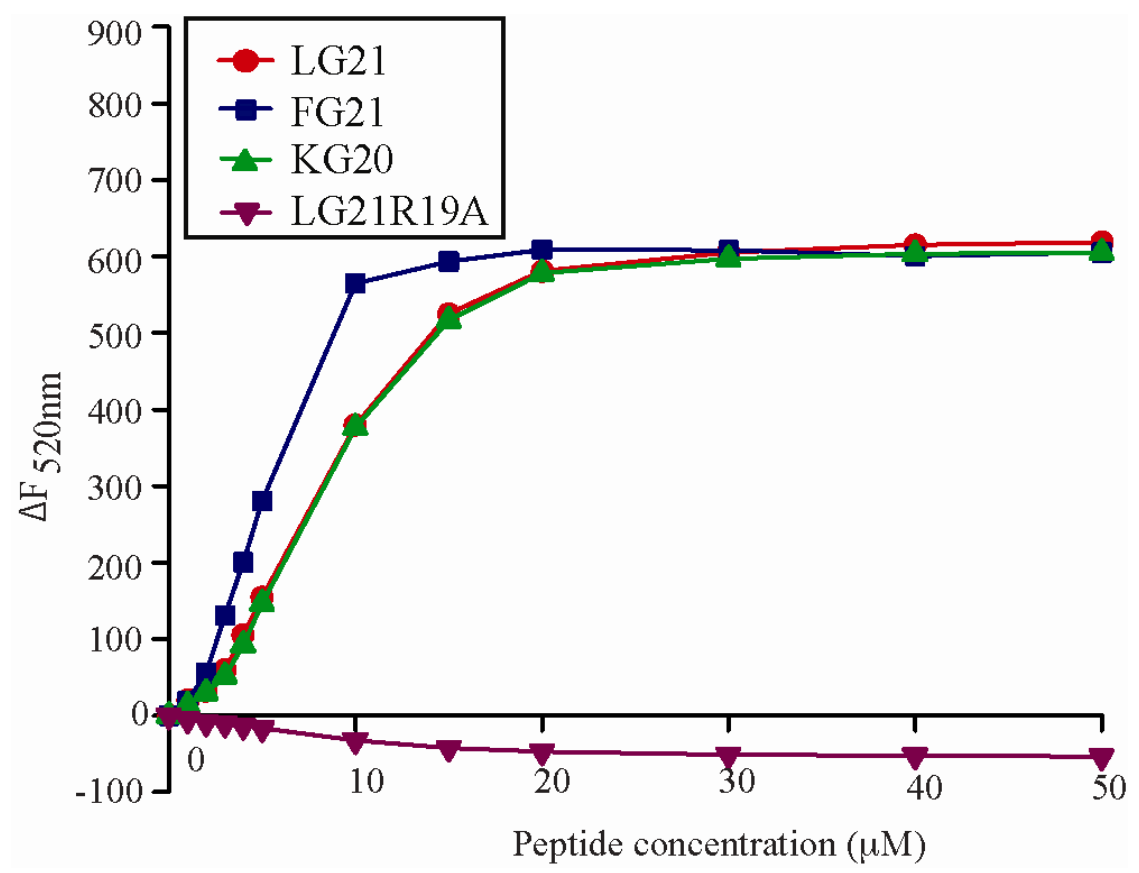

Figure 3.2-4: Complete membrane disrupting ability of hybrids and Ala mutant peptide in E.coli. $\Delta \mathbf{F}_{520 \mathrm{~mm}}$ is the difference between fluorescence intensities after and before addition of peptides at emission max of SYTOX green at $520 \mathrm{~nm}$.

The remarkably active hybrid peptides LG21, FG21 and KG20 can internalize the SYTOX green dye to nucleic acids by disrupting inner bacterial membrane also (Figure 3.2-4). At around $10 \mu \mathrm{M}$ of active peptide concentration itself, the peptides can effectively disrupt both the membranes completely thus permeabilizing the dye (Figure 3.2-4). Whereas, LG21R19A shows decrease in fluorescence intensity indicating very weak membrane disrupting ability.

\subsubsection{Membrane permeabilization invitro - Calcein leakage assay:}

Liposomes are considered as excellent membrane mimics and the release of entrapped calcein can be compared to the membrane permeabilization ability of peptides invitro [166]. The mammalian membranes are made up of zwitterionic lipids which is enacted by POPC liposomes and POPG, a negatively charged phospholipid is introduced in the ratio of 3:1 POPC: POPG as in bacterial membrane. 


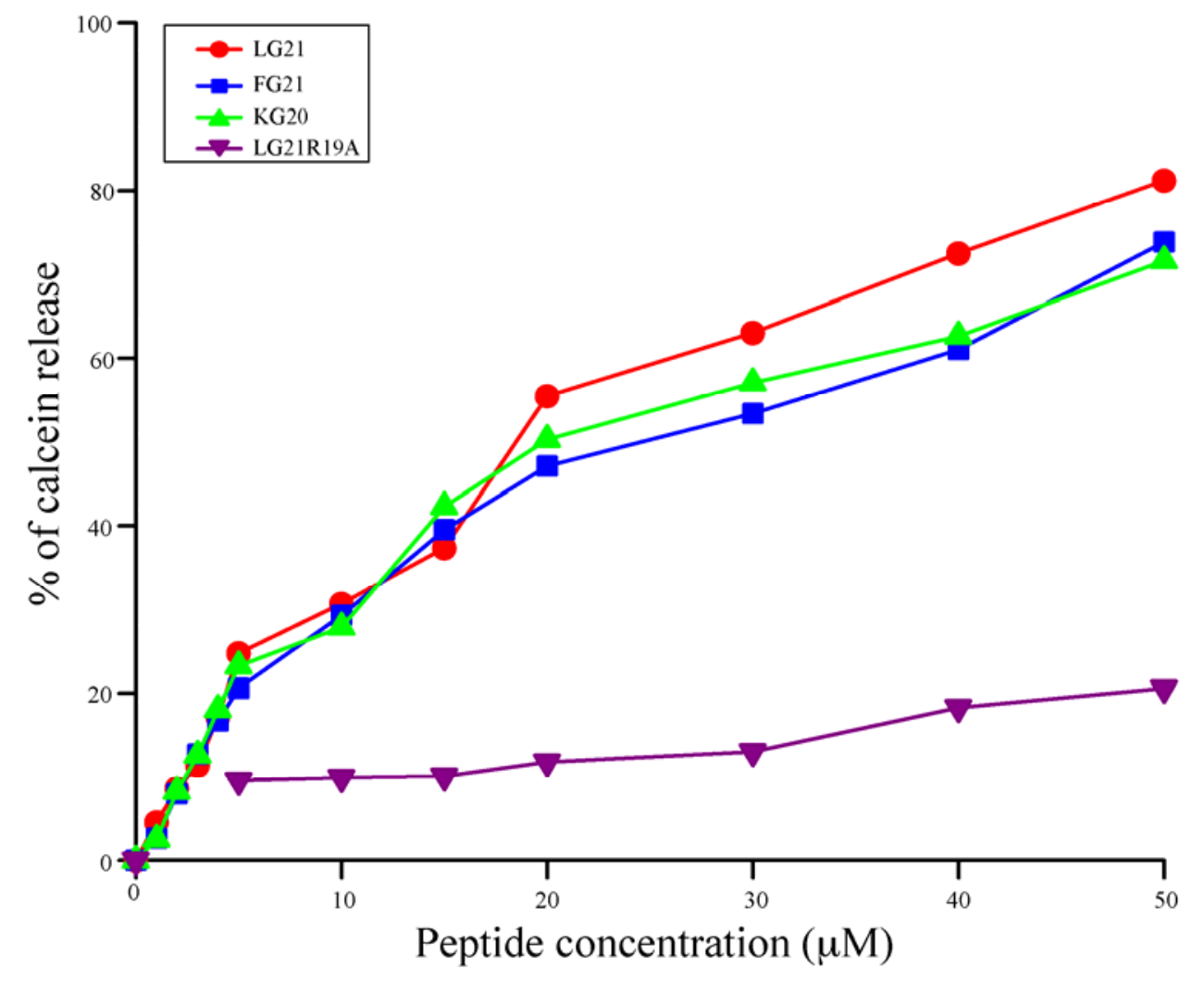

Figure 3.2-5: Membrane permeabilization invitro by hybrid and LG21Ala mutant peptides in POPC liposomes.

As can be seen from Figure 3.2-5, the leakage of entrapped calcein is limited to about $80 \%$ by active hybrid peptides in POPC liposomes. In other words, the active hybrid peptides, at concentrations several fold higher than their MIC values, show $80 \%$ of leakage from POPC liposomes. At concentrations of their MIC i.e. around 2-4 $\mu \mathrm{M}$, they show only $20 \%$ leakage of POPC liposomes (Figure 3.2-5). On the other hand, the inactive Ala mutant LG21R19A does not show any leakage from POPC liposomes (Figure 3.2-5). It could be either that this peptide completely lacks membrane disrupting ability irrespective of the charge on the membrane or this peptide does not lyse POPC liposomes. 

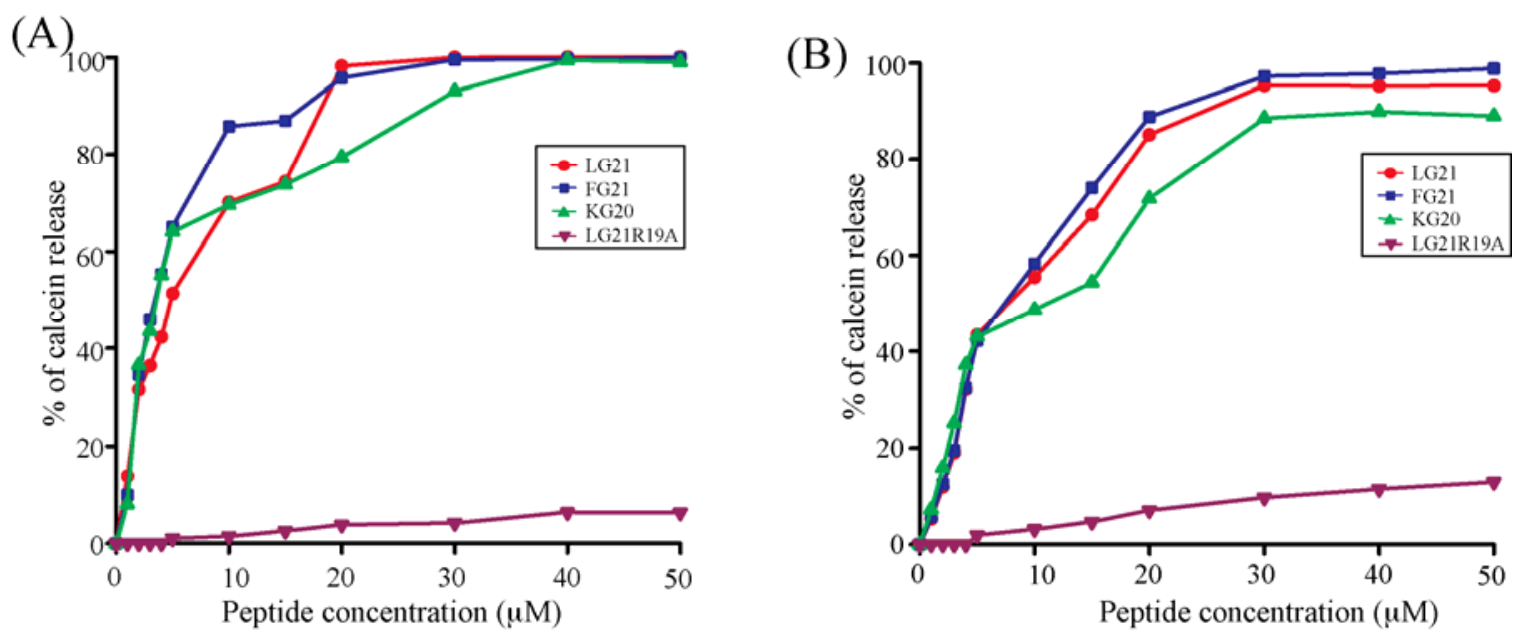

Figure 3.2-6: Membrane permeabilization invitro by hybrid and LG21Ala mutant peptides in (A) POPC: POPG (3:1) liposomes and (B) POPC:LPS (80:20 w/v) liposomes.

In the case of POPC: POPG (3:1) liposomes, with the introduction of negatively charged phospholipids, the competently active hybrids show 100\% leakage (Figure 3.2-6A). LG21R19A peptide does not show any remarkable change in leakage even with the inclusion of negatively charged POPG lipid. The hybrid peptides LG21, FG21 and KG20 show 50\% of leakage at their MIC concentrations itself (Figure 3.2-6A).

Inorder to confirm the specificity of conjugation of LPS binding motif to aggregating peptides, the liposome leakage was also carried out in POPC: LPS liposomes. As can be seen from Figure 3.2-6B, the active hybrids LG21, FG21 and KG20 can adequately leak calcein from liposomes. At around $5 \mu \mathrm{M}$ peptide concentration itself, $50 \%$ of calcein is released. The positive charges in the WKRKRF motif help the peptide to get electrostatically attracted towards the negatively charged bacterial membrane and the hydrophobicity exerted by other hydrophobic aminoacids disrupt the lipid packing. Whereas the mutation in one of these cationic residues to Ala in LG21R19A has completely disrupted the membrane binding ability and hence does not show leakage of calcein in any of the liposome models. (Figure 3.2-5, Figure 3.2-6). 


\subsubsection{Membrane depolarization:}

There exists a membrane potential between internal and external compartments of the biological membrane. Fluorescent probes like cyanine dyes can be used to study this membrane potential. The cell is said to be hyperpolarized when the intensity of the dye decreases i.e. when the dye gets compartmentalized between the membranes and the membrane is said to be depolarized when the membrane potential is dissipated resulting in increase in intensity [167]. The membrane dissipation potential of the peptides were detected in intact E.coli cells and spheroplasts, where the cell wall is removed by means of osmotic shock procedure as described [162].
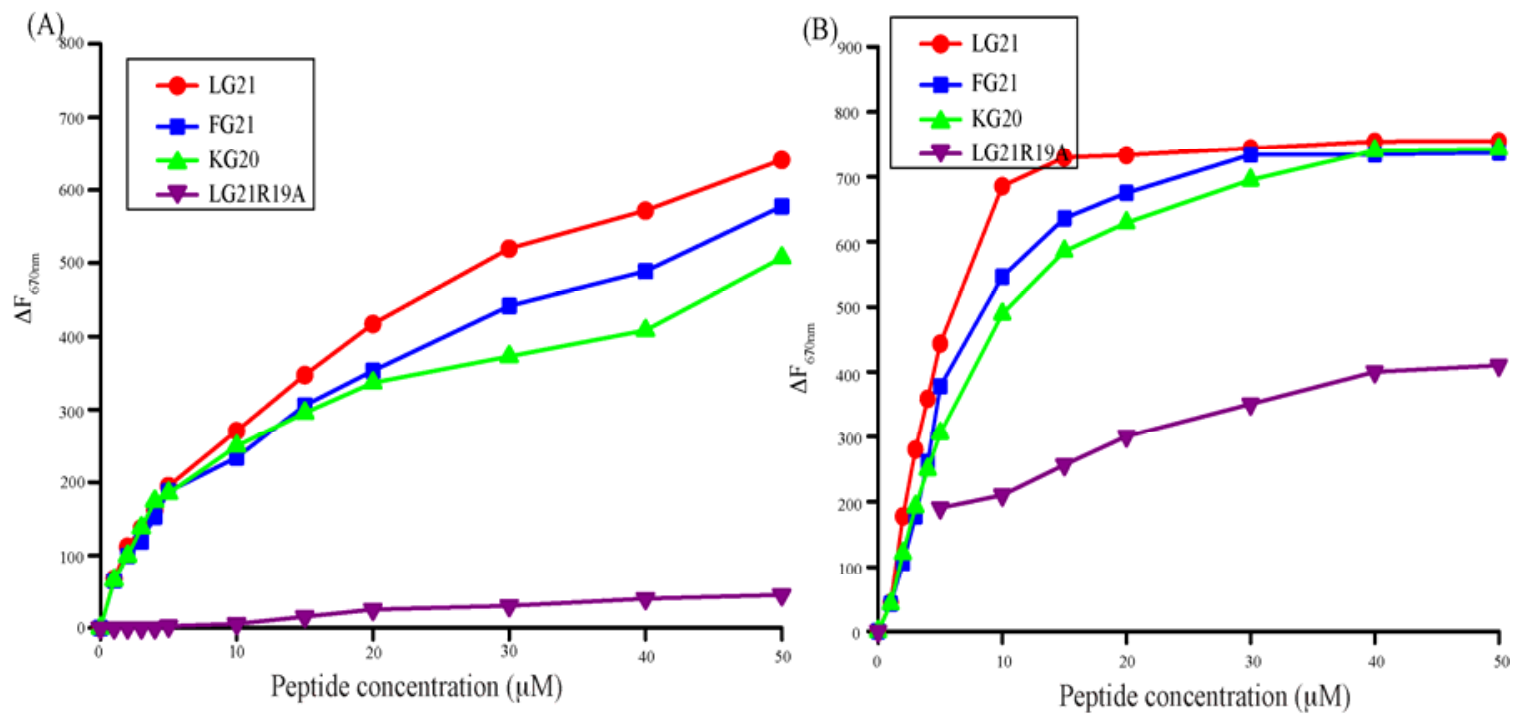

Figure 3.2-7: Membrane depolarization of (A) intact E.coli and (B) spheroplasts by hybrid peptides. $\Delta F_{620 \mathrm{~nm}}$ is the difference between fluorescence intensities after and before addition of peptides.

After the dye is hyperpolarized, the ability of the hybrid peptides to depolarize the membrane potential and thereby increase in fluorescence intensity was measured. In intact E.coli cells, the hybrid peptides LG21, FG21 and KG20 show marked increase in intensity when compared to LG21R19A (Figure 3.2-7A).

When hyperpolarized E.coli spheroplasts were added with increasing concentration of peptides, there was an increase in intensity for all of the peptides. LG21, FG21 and KG20 show marked increase in intensity followed by LG21R19A peptide (Figure 3.2-7B). It is clearly evident from these experimental results that the property of 
aggregation of temporins on LPS layer is successfully overcome by the conjugation of LPS binding motif. Hence the conjugated peptides, regardless of E.coli membrane arrangement, can dissipate the membrane potential.

\subsubsection{Intrinsic tryptophan fluorescence measurements:}

Tryptophan is often considered as an excellent intrinsic fluorescent probe as it is sensitive to the polarity of the environment.

Table 3.2-9: Difference in the emission maximum of Trp fluorescence ( $\Delta \lambda \max )$ in the aqueous environment and in micelles.

\begin{tabular}{|l|l|l|l|}
\hline \multirow{2}{*}{ Peptides } & \multicolumn{3}{|c|}{$\Delta \lambda \max$} \\
\cline { 2 - 4 } & LPS & DPC & SDS \\
\hline LG21 & 20 & 14 & 18 \\
\hline FG21 & 18 & 10 & 22 \\
\hline KG20 & 18 & 14 & 18 \\
\hline LG21R19A & 2 & 12 & 10 \\
\hline
\end{tabular}

In aqueous environment, if the $\operatorname{Trp}$ residue is completely exposed, the emission maximum would be around $354-358 \mathrm{~nm}$. But if the Trp is buried in hydrophobic ambience of the solvent, the emission maximum decreases to $330-335 \mathrm{~nm}$ i.e. experiences a blue shift. 

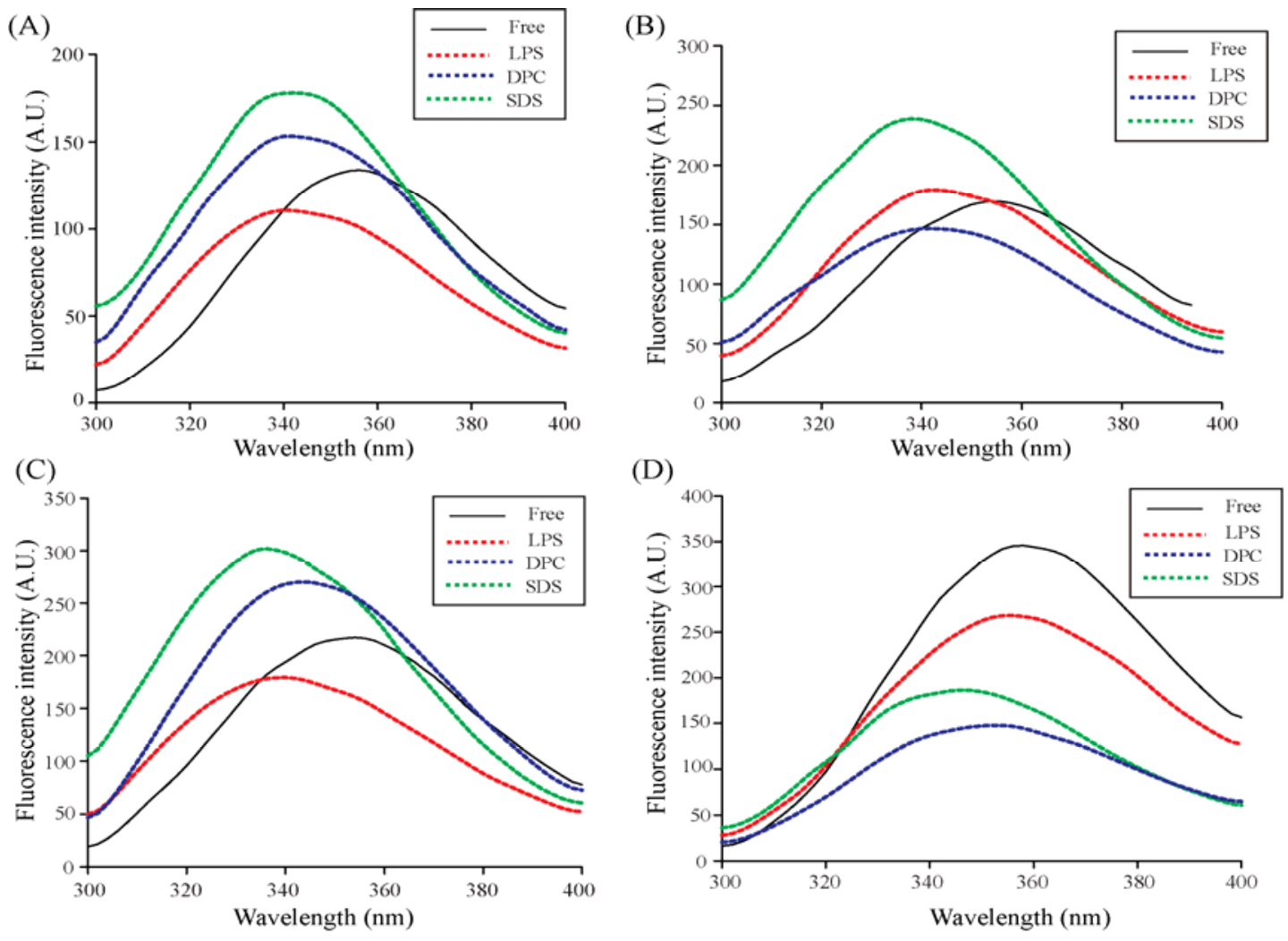

Figure 3.2-8: Intrinsic tryptophan fluorescence measurement of (A) LG21, (B) FG21, (C) KG20 and (D) LG21R19A in aqueous state (black straight line), LPS micelles (red dots), DPC micelles (blue dots) and SDS micelles (green dots).

The Trp emission maxima of hybrid peptides were around 356-360 $\mathrm{nm}$ in aqueous environment confirming that the $\operatorname{Trp}$ is exposed to the solvent (Figure 3.2-8). When the active peptides LG21, FG21 and KG20 were titrated with increasing concentrations of negatively charged micelles LPS and SDS, the Trp emission experienced blue shift (Figure 3.2-8 A, B, C) confirming the binding of the peptides. LG21 peptide showed highest change in emission maxima followed by FG21 and KG20 (Table 3.2-9). But the inactive mutant LG21R19A show very less or no shift at all in the presence of LPS micelle, but did show blue shift in the presence of SDS micelles (Figure 3.2-8 D, Table 3.2-9). When these peptides were titrated with zwitterionic DPC micelles, all the peptides did show blue shift but to lesser extend when compared to LPS and SDS micelle (Table 3.2-9). 


\subsubsection{Acrylamide quenching:}

The extent of binding of peptides to lipid micelles can be determined by the availability of Trp residue to neutral quencher acrylamide. The Stern-Volmer $\left(\mathrm{K}_{\mathrm{sv}}\right)$ constant is very high when Trp is exposed to the environment, which undergoes drastic reduction when Trp is buried in the hydrophobic surrounding. The $\mathrm{K}_{\mathrm{sv}}$ values of the peptides in aqueous media were as high as 15.6, 25.4 and 27.9 for LG21, FG21 and KG20 respectively confirming the exposure of tryptophan to aqueous environment.

Table 3.2-10: Stern-Volmer $\left(K_{s v}\right)$ quenching constants of the hybrid peptides in aqueous state and micelle bound state.

\begin{tabular}{|l|l|l|l|l|}
\hline Peptides & \multicolumn{3}{|l|}{$\begin{array}{l}\text { Acrylamide } \\
\left(\mathrm{K}_{\text {sv }}\right)\end{array}$} \\
\cline { 2 - 5 } & Free & LPS & DPC & SDS \\
\hline LG21 & 15.6 & 4.7 & 7.3 & 5.1 \\
\hline FG21 & 25.4 & 7.7 & 3.6 & 6.0 \\
\hline KG20 & 27.9 & 5.8 & 6.7 & 5.8 \\
\hline LG21R19A & 17.0 & 10.9 & 6.5 & 6.3 \\
\hline
\end{tabular}

In the presence of negatively charged micelles LPS and SDS, $\mathrm{K}_{\mathrm{sv}}$ values experienced tremendous decrease suggesting that the tryptophan residue is buried into hydrophobic environment (Table 3.2-10). The Ksv values decrease in the presence of DPC micelles also, but not as low as negatively charged micelles (Table 3.2-10). Hence the interaction between peptides and DPC micelles could be confined to surface. LG21R19A exhibited very weak binding with LPS and hence very high $\mathrm{K}_{\mathrm{sv}}$ value when compared to SDS and DPC micelles (Table 3.2-10).

\subsubsection{Isothermal Titration Calorimetry:}

The thermodynamic principles that govern the binding of antimicrobial peptides to LPS were studied using Isothermal titration calorimetry. The parameters determined are outlined in Table 3.2-11. The binding profiles of the hybrid peptides with LPS 
micelles are compiled in Figure 3.2-9. In this experiment, peptide is injected into LPS micelles to detect the binding interactions. In an equilibrium state, the peptide should bind with LPS and then dissociate. The rate of dissociation of peptide $\left(\mathrm{K}_{\mathrm{d}}\right)$ from LPS can be directly taken as a count for the extent of binding. The lesser the $K_{d}$ value, the more tighter will be the binding. In the case of active hybrid peptides, LG21, FG21 and $\mathrm{KG} 20$, the interactions are endothermic i.e. heat is released and entropy driven as defined with upward movement of the peaks (Figure 3.2-9 A, B, C). The $\mathrm{K}_{\mathrm{d}}$ values were also in nanomolar range confirming that the peptide is binding very tightly to LPS (Table 3.2-11).

Table 3.2-11: Thermodynamic parameters for hybrid peptides binding to LPS micelles determined by Isothermal titration calorimetry.

\begin{tabular}{|l|l|l|l|l|}
\hline & LG21 & FG21 & KG20 & LG21R19A \\
\hline $\mathrm{K}_{\mathrm{a}}\left(\mu \mathrm{M}^{-1}\right)$ & 1.64 & 1.6 & 3.2 & 0.099 \\
\hline$\Delta \mathrm{H}\left(\mathrm{kcal}^{\mathrm{m}} \mathrm{mol}^{-1)}\right.$ & 0.58 & 4.4 & 3.4 & -1.7 \\
\hline $\mathrm{T} \Delta \mathrm{S}\left({\left.\mathrm{kcal} . \mathrm{mol}^{-1} \mathrm{deg}^{-1}\right)}^{-1}\right.$ & 8.9 & 12.8 & 12.2 & 5.1 \\
\hline$\Delta \mathrm{G}\left(\mathrm{kcal} . \mathrm{mol}^{-1}\right)$ & -8.4 & -8.4 & -8.8 & -6.8 \\
\hline $\mathrm{K}_{\mathrm{d}}(\mu \mathrm{M})$ & 0.6 & 0.6 & 0.3 & 10 \\
\hline
\end{tabular}

The thermodynamic parameters for the inactive mutant LGR19A were also determined. This mutant seem to show much weaker affinity to LPS and its binding profile mainly revealed an exothermic reaction as indicated by the downward movement of the peaks (Figure 3.2-9, D).

An endothermic or entropy driven reaction indicates predominant role of hydrophobic interactions. Whereas an exothermic or enthalpy driven reaction indicates the electrostatic attraction. Hence it is clearly evident from the reactions that the active peptides gets attracted to LPS electrostatically and its dissociation is successfully brought about by the hydrophobic interactions as indicated with endothermic profile of binding (Figure 3.2-9 A, B, C). On the other hand, the inactive mutant peptide was electrostatically attracted but the threshold hydrophobicity required for dissociating the LPS micelles was not achieved that results in its inability to kill bacterial cells. 

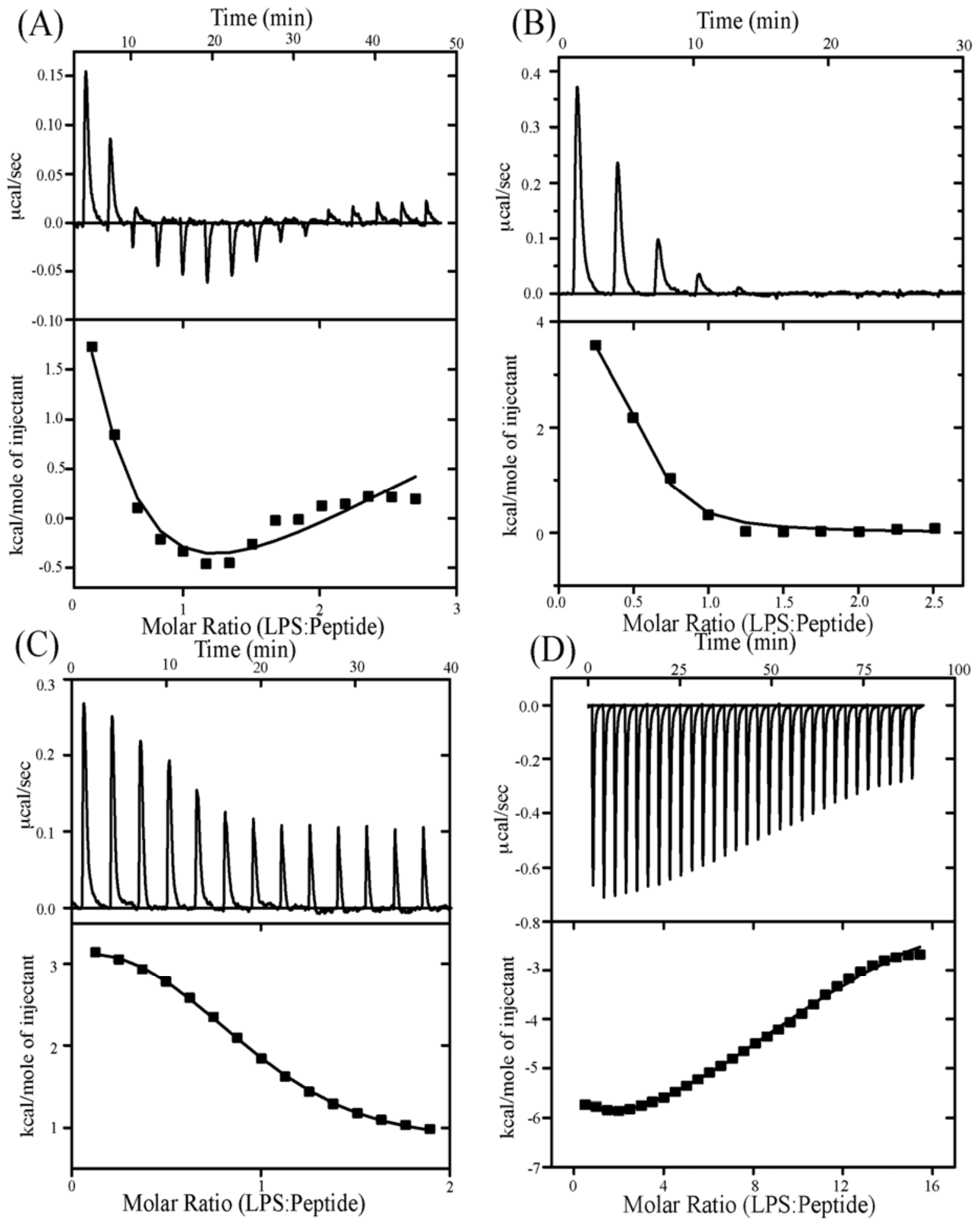

Figure 3.2-9: Binding profiles of hybrid peptides (A) LG21, (B) FG21, (C) KG20 and (D) LG21R19A with LPS micelles at $25^{\circ} \mathrm{C}$ by Isothermal titration calorimetry. 


\subsubsection{Dynamic Light Scattering measurements:}

Lipopolysaccaharides form micelles of various sizes when dissolved in aqueous solutions and micelles are regarded as active form of LPS.
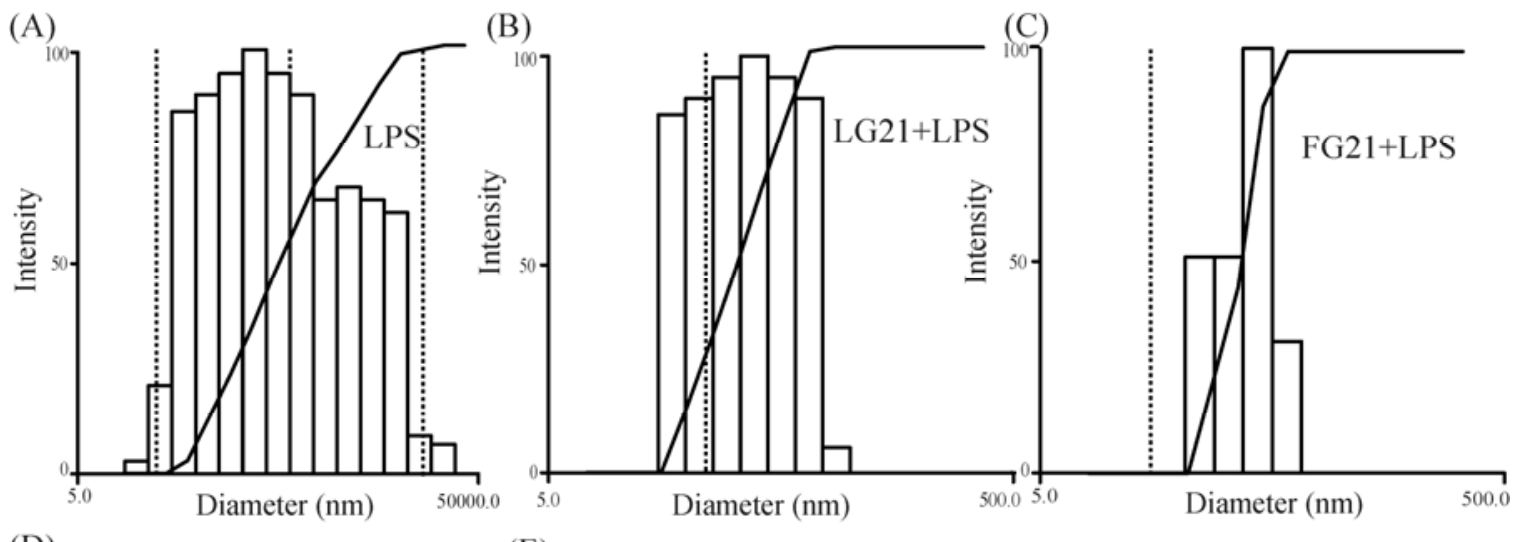

(D)

(E)
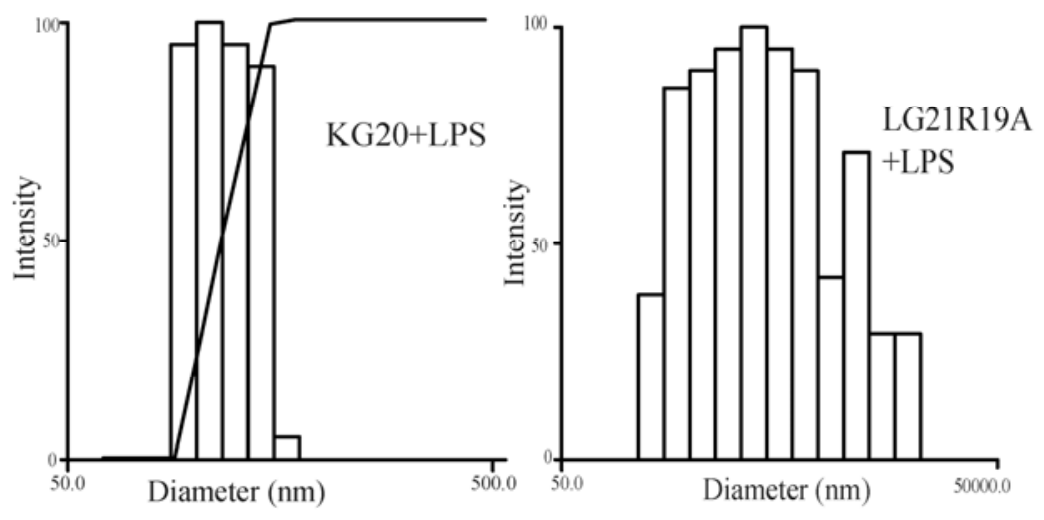

Figure 3.2-10: LPS micelle dissociation by hybrid peptides as monitored with Dynamic light scattering experiments. (A) $0.5 \mu \mathrm{M}$ LPS, (B) LPS:LG21 (1:4), (C) LPS:FG21 (1:4), (D) LPS:KG20 (1:4), (E) LPS:LG21R19A (1:4).

The ability of conjugated hybrid peptide to dissociate these LPS micelles were monitored with DLS measurements. When $0.5 \mu \mathrm{M}$ of LPS alone was analysed with DLS measurements, the particle size distribution of LPS micelles was larger and about $806 \mathrm{~nm}$ size was predominantly present in $100 \%$ (Table 3.2-12, Figure 3.2-10, A). In the presence of increasing concentration of LG21, FG21 and KG20 peptides, the particle size reduced dramatically in the range of 5.0-500.0 nm (Figure 3.2-10 B, C, D, Table 3.2-12). When $2 \mu \mathrm{M}$ of peptides were added, the sizes drastically reduced to 85 $\mathrm{nm}, 87 \mathrm{~nm}$ and $134 \mathrm{~nm}$ respectively (Table 3.2-12). This result comes as a great 
evidence for the efficient dissociation of LPS micelles by conjugated hybrid peptides. LG21R19A show very limited dissociation of LPS micelles with $521 \mathrm{~nm}$ size in 100\% intensity. A marked increase in the distribution of micelle particles i.e. from $50 \mathrm{~nm}-$ $50,000 \mathrm{~nm}$ was also noted (Figure 3.2-10E).

Table 3.2-12: Dissociation of variously sized LPS micelles to smaller particles by hybrid peptides.

\begin{tabular}{|l|l|}
\hline & Diameter $(\mathrm{nm})$ \\
\hline LPS $(0.5 \mu \mathrm{M})$ & 806 \\
\hline LPS:LG21 (1:4) & 85 \\
\hline LPS:FG21 (1:4) & 87 \\
\hline LPS:KG20 (1:4) & 134 \\
\hline LPS:LG21R19A(1:4) & 521 \\
\hline
\end{tabular}

\subsubsection{Dissociation of FITC conjugated LPS:}

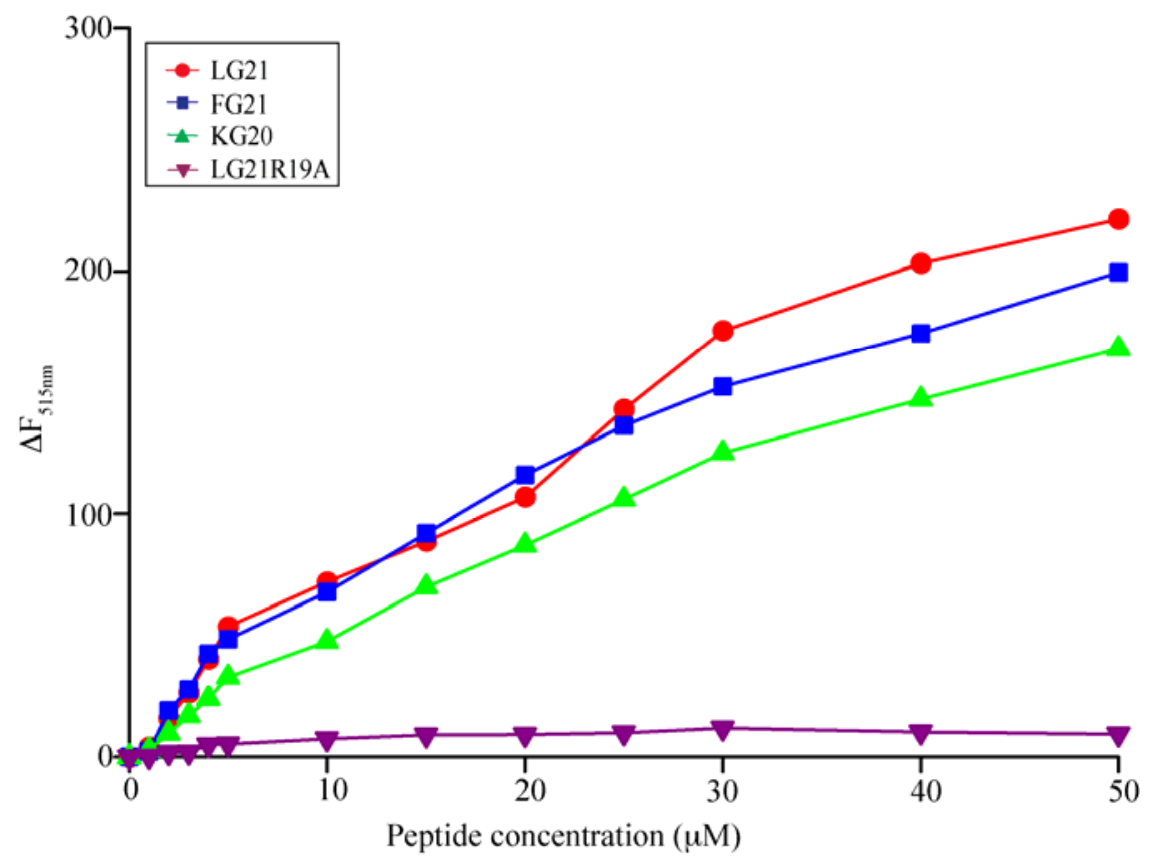

Figure 3.2-11: Dissociation of FITC conjugated LPS by active hybrids LG21, FG21, KG20 and inactive LG21R19A. 
LPS exists in aggregates or micelle forms in aqueous solutions. When FITC conjugated LPS is dissolved in buffer, LPS forms micelles in such a way that FITC is packed in the LPS micelles. Hence the fluorescence of FITC is quenched in such micelles and dequenched only when the LPS micelles are dissociated. The ability of the hybrid peptides to dequench FITC was analysed by fluorescence spectrophotometer.

As can be seen from Figure 3.2-11, there is marked increase in intensity with increase in peptide concentrations. All of the three active hybrids LG21, FG21 and KG20 showed gradual dissociation of LPS micelles thereby dequenching FITC. On the other hand, LG21R19A did not show any increase in fluorescent intensity (Figure 3.2-11). This result is in close agreement with DLS measurements where addition of more peptides to LPS micelles resulted in complete dissociation of big particle size LPS micelles to smaller particles (Table 3.2-12).

\subsubsection{Transmission Electron Microscopy studies:}

(A)

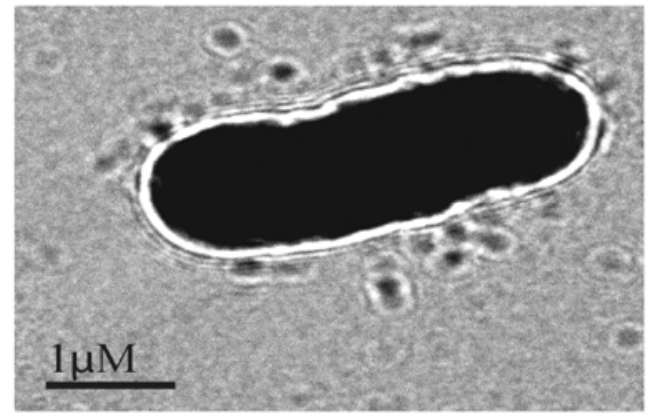

(C)

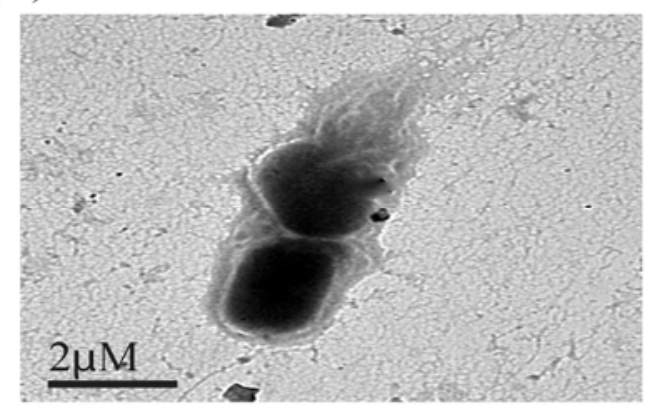

(B)

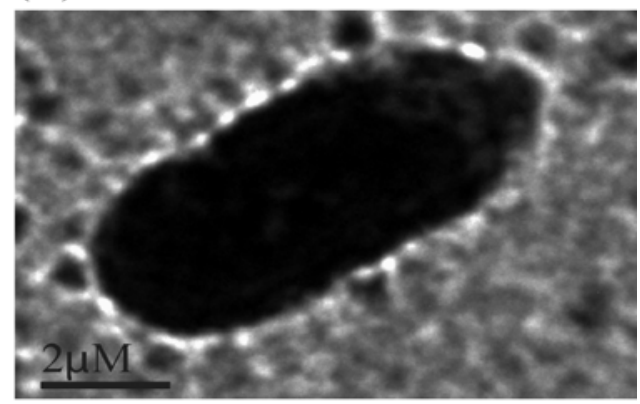

(D)

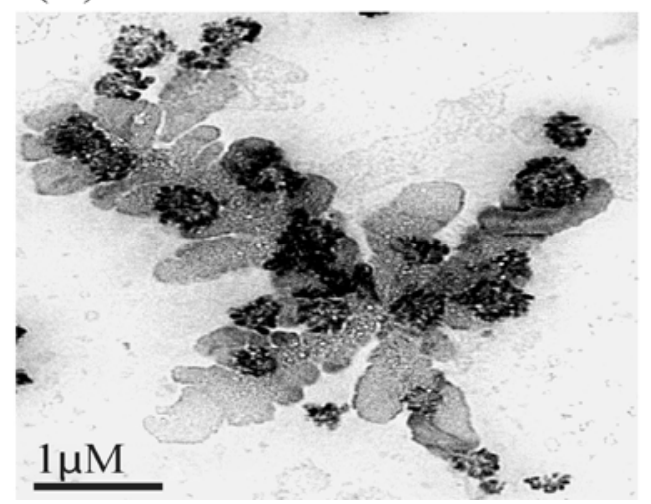

Figure 3.2-12: Electron micrographs of E.coli treated with (A) $0 \mu$ M LG21, (B) 3 $\mu$ M LG21, (at MIC) (C) $8 \mu$ M LG21 and (D) $15 \mu$ M LG21, (above MIC). 
Mid-log phase grown E.coli cells were treated with different concentrations of LG21 for 30 minutes followed by staining and visualization with TEM. Control reaction without any peptide served as negative control. The electron micrographs in Figure 3.2-12 A shows the continuous membrane formation of intact E.coli cell. Further treatment of the cells with LG21 has gradually disrupted the membrane and finally the cells completely (Figure 3.2-12). At MIC concentration i.e. $3 \mu \mathrm{M}$, blebbing of membrane was observed (Figure 3.2-12, B) followed by the membrane rupture and release of cellular contents at concentration just above MIC (8 $\mu \mathrm{M})$ (Figure 3.2-12). At concentrations several fold above MIC $(15 \mu \mathrm{M})$ the cells were completely disrupted by LG21 and only debris can be viewed (Figure 3.2-12, D).

\subsubsection{Structural characterization:}

\subsubsection{Circular Dichroism spectrometry:}

The secondary structure of the peptides in aqueous solution and micelle bound form was obtained from Circular Dichroism measurements. The far-UV CD spectra of all the hybrid peptides in aqueous solution delineate strong negative minima at around $195 \mathrm{~nm}$. This is characteristic of random coil conformation (Figure 3.2-13). But the peptides underwent highly ordered conformational change as they were titrated with negatively charged LPS, SDS micelles and zwitterionic DPC micelles. A positive maxima peak at $195 \mathrm{~nm}$ and two negative minima peaks at $208 \mathrm{~nm}, 222 \mathrm{~nm}$ are typical representation of helical conformations.

All of the hybrid peptides, irrespective of its activity demonstrated the conformational change from random coil to alpha helix. Also regardless of the micelle environment, all the peptides adopted alpha helical conformation (Figure 3.2-13). This structure determination demonstrates that the binding, conformational change and activity are different parameters and are independent of each other. 
(A)

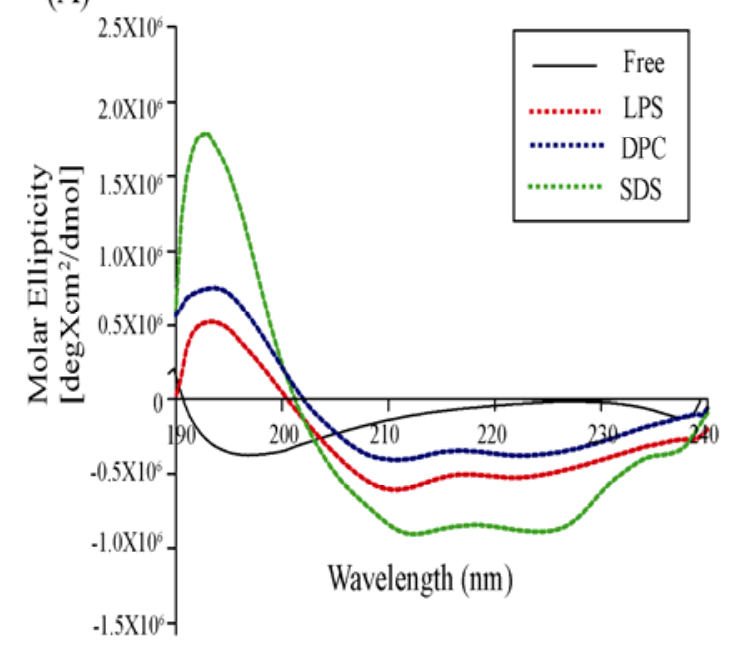

(C)

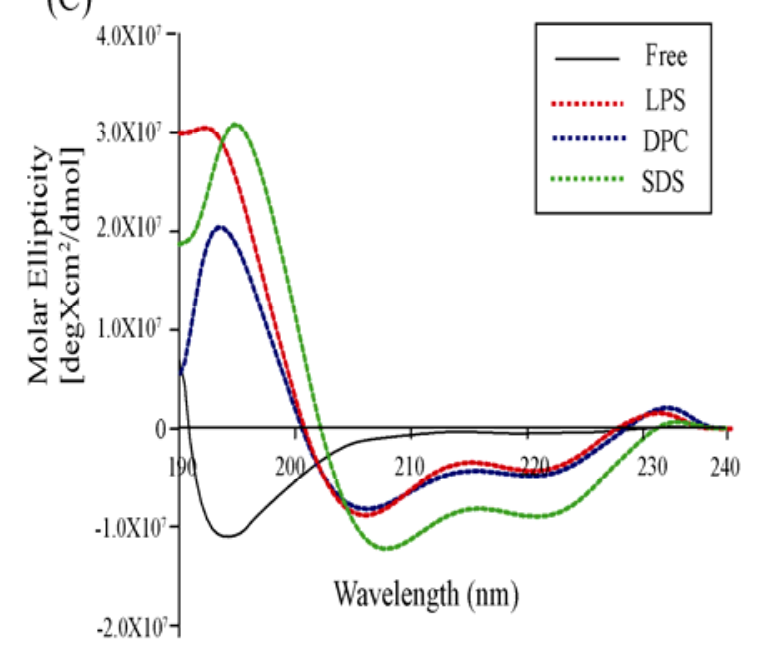

(B)
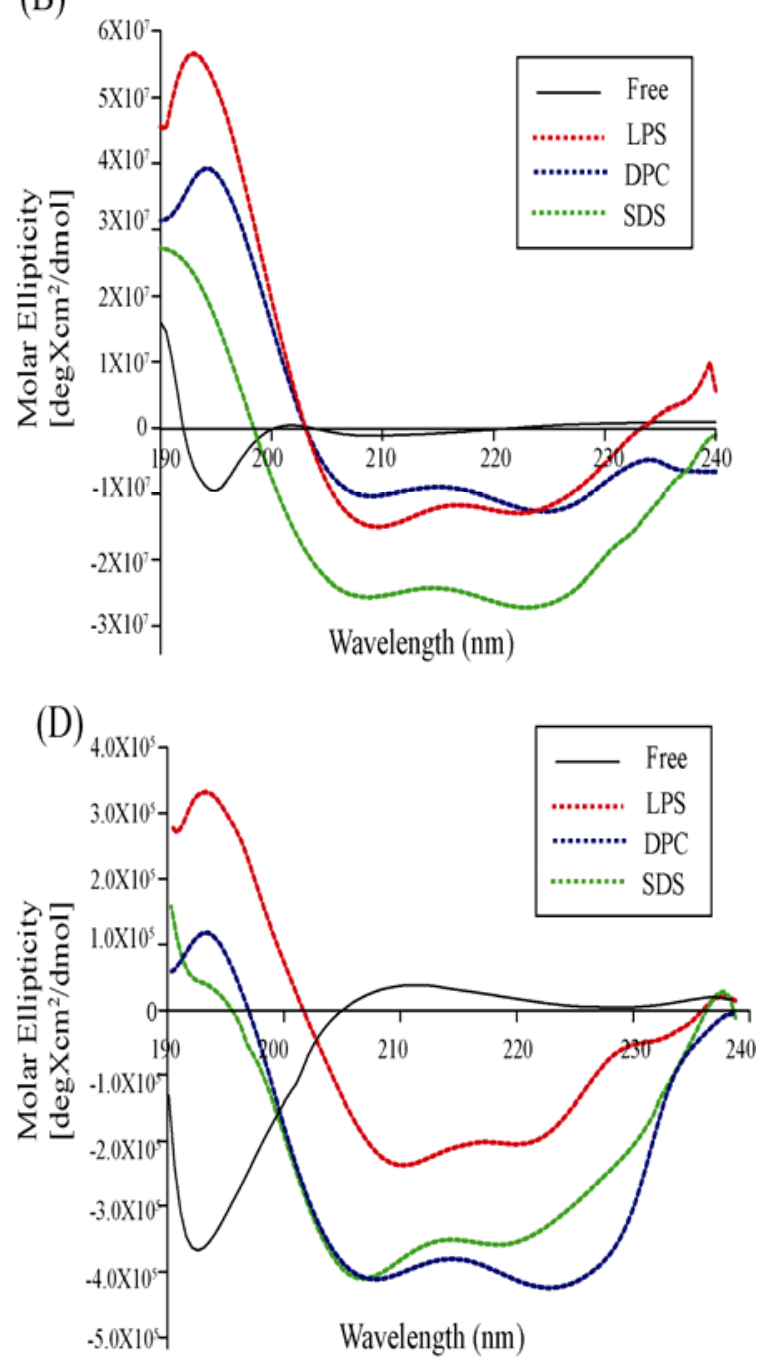

Figure 3.2-13: Far-UV CD spectra of (A) LG21, (B) FG21, (C) KG20, (D) LG21R19A in aqueous solution (black straight line), negatively charged LPS (red dot), SDS (green dot) and zwitterionic DPC (blue dot) micelles.

\subsubsection{Nuclear Magnetic Resonance Spectroscopy:}

NMR has been a convenient tool to study antimicrobial peptide interactions with lipid membranes. The solution conformations of the active hybrid peptide LG21 and inactive mutant LG21R19A in aqueous solution and DPC micelles were obtained from two dimensional TOCSY and NOESY experiments. 


\subsubsection{NMR studies of LG21 and LG21R19A in the presence of LPS micelles:}

The conformation of the peptides in aqueous solution was acquired using two dimensional ${ }^{1} \mathrm{H}-{ }^{1} \mathrm{H}$ TOCSY and NOESY experiments. Sequential walking of specific spins of the aminoacids was achieved through the overlay of TOCSY and NOESY spectra. In aqueous solution, both the peptides LG21 and LG21R19A showed only sequential NOEs $\left(\mathrm{C}^{\mathrm{a}} \mathrm{H} / \mathrm{NH}\right)$. Absence of medium range and long range NOEs indicate the peptides does not have ordered conformation and appear as random coil in aqueous state.

(A)

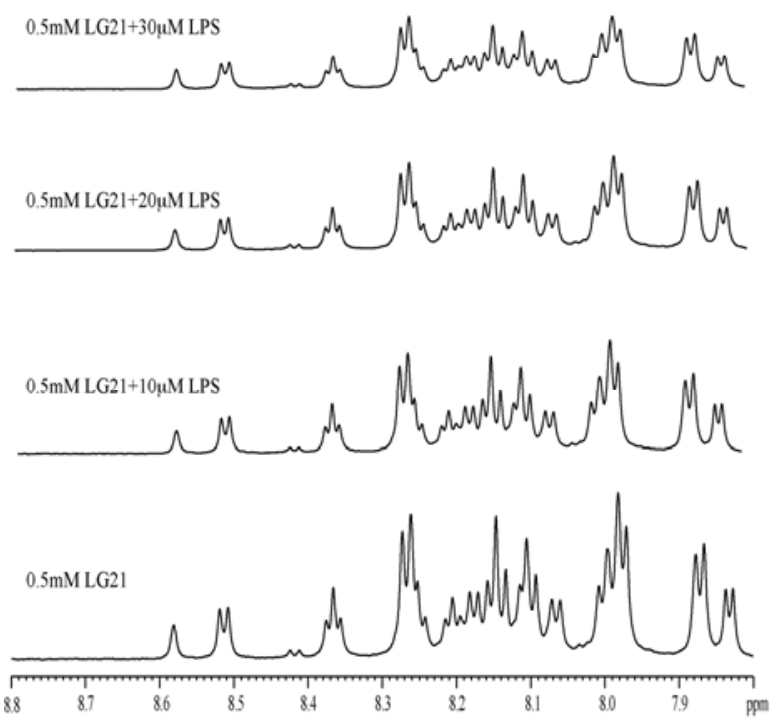

(B)
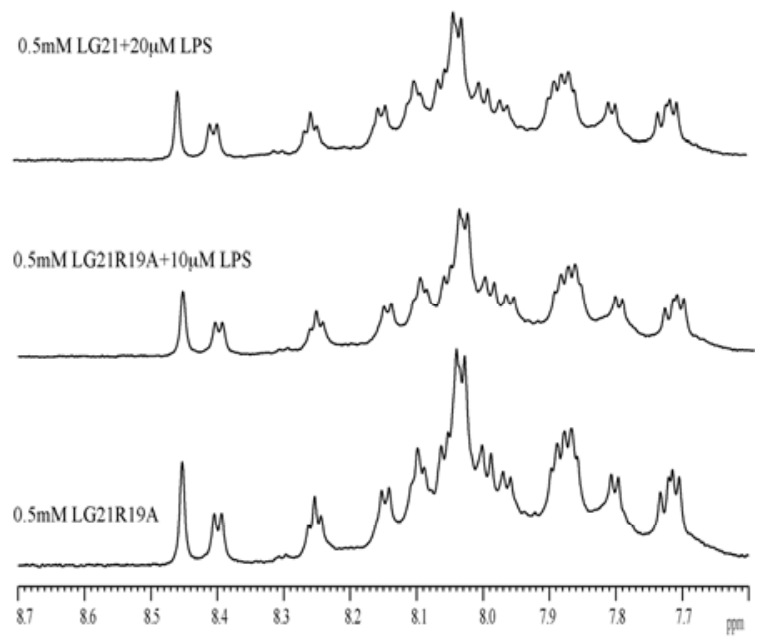

Figure 3.2-14: Low field amide region of 1-D proton spectra of (A) LG21 and (B) LG21R19A in the presence of increasing concentration of LPS.

Tr-NOESY experiments have been proved to be a useful method to study the conformational change of the peptides in LPS micelles. The property of LPS forming micelles of higher molecular weight when dissolved in aqueous solutions was taken advantage in acquiring tr-NOE with peptides. If the peptides are binding to LPS in NMR time scale or with moderate affinity, then there will be line broadening of the resonances. So LPS from $1 \mathrm{mM}$ stock was added to LG21 and LG21R19A peptide solutions in concentration range of $10 \mu \mathrm{M}$. No significant line broadening was observed even after the addition of $30 \mu \mathrm{M}$ LPS (Figure 3.2-14). It should be recalled that LG21 binds with LPS very tightly with $\mathrm{K}_{\mathrm{d}}$ value of $0.6 \mu \mathrm{M}$ (Table 3.2-11). In 
order to observe tr-NOEs, the chemical exchange should be fast or intermediate in NMR time scale [25]. Hence after addition of $30 \mu \mathrm{M}$ of LPS, two dimensional ${ }^{1} \mathrm{H}-{ }^{1} \mathrm{H}$ NOESY and TOCSY spectra of LG21 was obtained with $150 \mathrm{~ms}$ and $80 \mathrm{~ms}$ respectively.

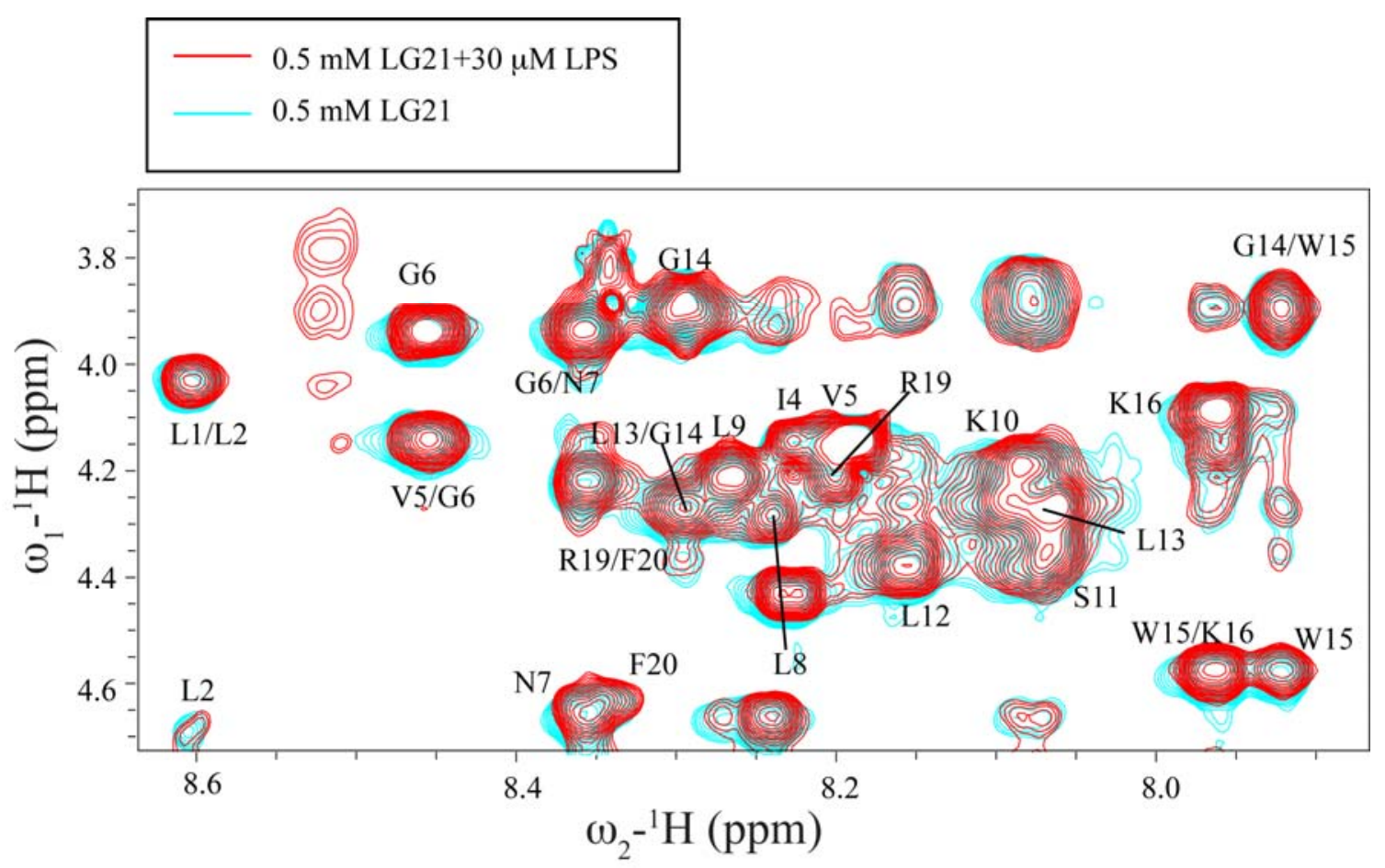

Figure 3.2-15: Overlay of two-dimensional ${ }^{1} \mathrm{H}-{ }^{1} \mathrm{H}$ NOESY spectra of $0.5 \mathrm{mM}$ LG21 in aqueous solution (Cyan) and 0.5 mM LG21 with $30 \mu$ M LPS (Red).

When NOESY spectra of LG21 in aqueous form and tr-NOESY spectra of LPS added LG21 were overlaid and analysed, only sequential NOEs ( $i$ to $i+1)$ were observed (Figure 3.2-15). This is in parallel with no broadening effect observed in 1-D experiment. Even though no structural information can be obtained from LPS binding experiment in NMR owing to peptide's strong binding to LPS, a striking effect on tryptophan's indole ring proton was observed in 1-D experiement. The signal from indole ring protons of tryptophan appears around $10.00 \mathrm{ppm}$ in 1-D NMR spectrum. In the case of active LG21, when increasing concentration of LPS was titrated, there was gradual shift towards downfield in indole ring signal of about $2.00 \mathrm{~Hz}$ (Figure 3.2-16, A). On the other hand, no such shift of indole ring protons was observed for 
LG21R19A when titrated against LPS (Figure 3.2-16, B). This is because when the active peptide, LG21 binds to LPS, the indole ring protons undergo chemical exhange with the conformational states and hence the peak intensity decreases with increase in LPS additions.
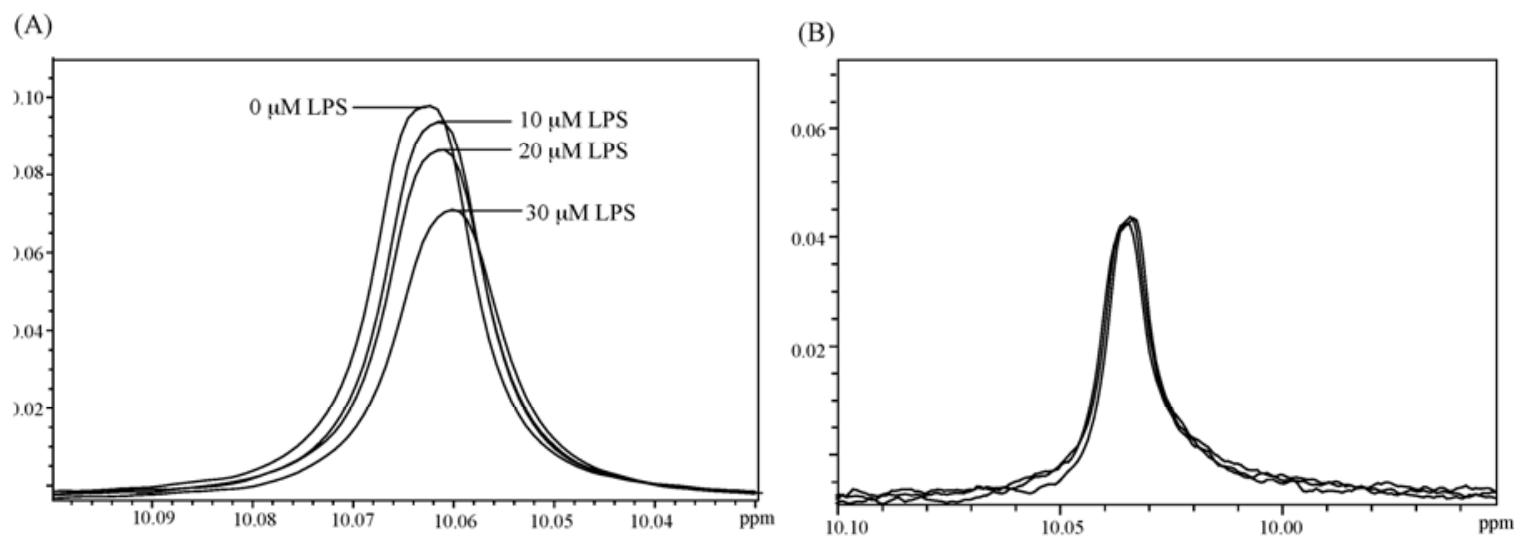

Figure 3.2-16: Comparison of the shift in indole ring proton of tryptophan with addition of increasing concentration of LPS in (A) LG21 and (B) LG21R19A.

\subsubsection{Structural studies of LG21 in DPC micelles:}

Due to lack of tr-NOEs in LPS micelles, $0.5 \mathrm{mM}$ LG21 was dissolved in $125 \mathrm{mM}$ deuterated DPC (DPC- $\mathrm{d}_{38}$ ) and two dimensional ${ }^{1} \mathrm{H}-{ }^{1} \mathrm{H}$ TOCSY and NOESY spectra were obtained. In this case, DPC concentration is several folds higher than peptide concentration expecting that the peptide be in completely bound form. As expected, LG21 in the presence of DPC micelles endowed a well resolved spectrum with more diagnostic NOEs that can be used for structure calculations.

Detailed analysis of DPC bound LG21 spectrum was carried out with extensive assignment of almost all of the peaks. The TOCSY spectrum appeared well resolved which helped to assign the specific spin system for $\mathrm{C}^{\mathrm{a}} \mathrm{H} / \mathrm{NH}$ region (Figure 3.2-17). All the aminoacids from L2 to G21 was identified except L1 and P3. The assignment of backbone NH/NH also delineates almost all of the sequential connectivity (Figure 3.2-18). 


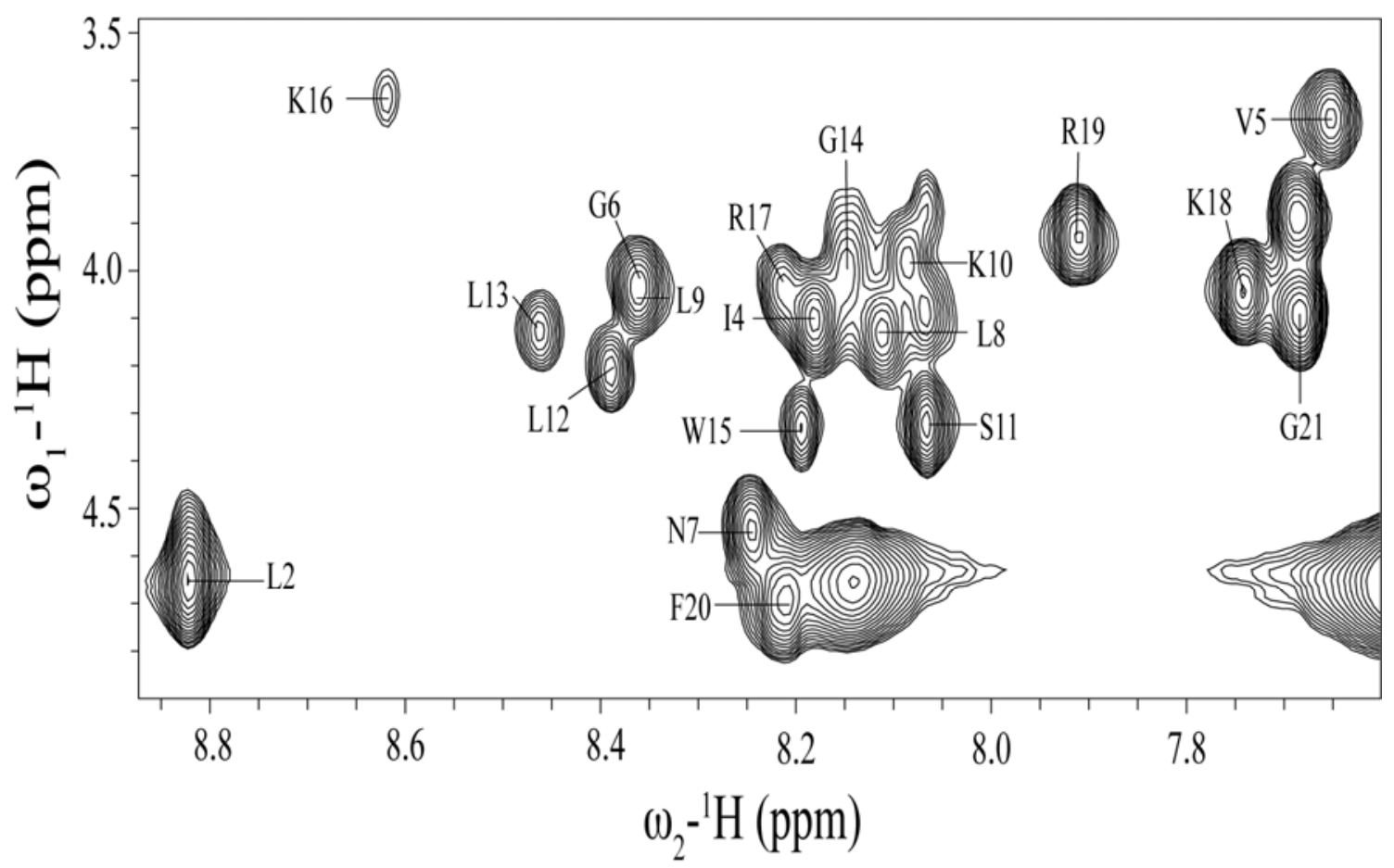

Figure 3.2-17: Sequence specific resonance assignment in the finger print region of two dimensional ${ }^{1} \mathrm{H}-{ }^{1} \mathrm{H}$ TOCSY spectrum of DPC bound LG21.

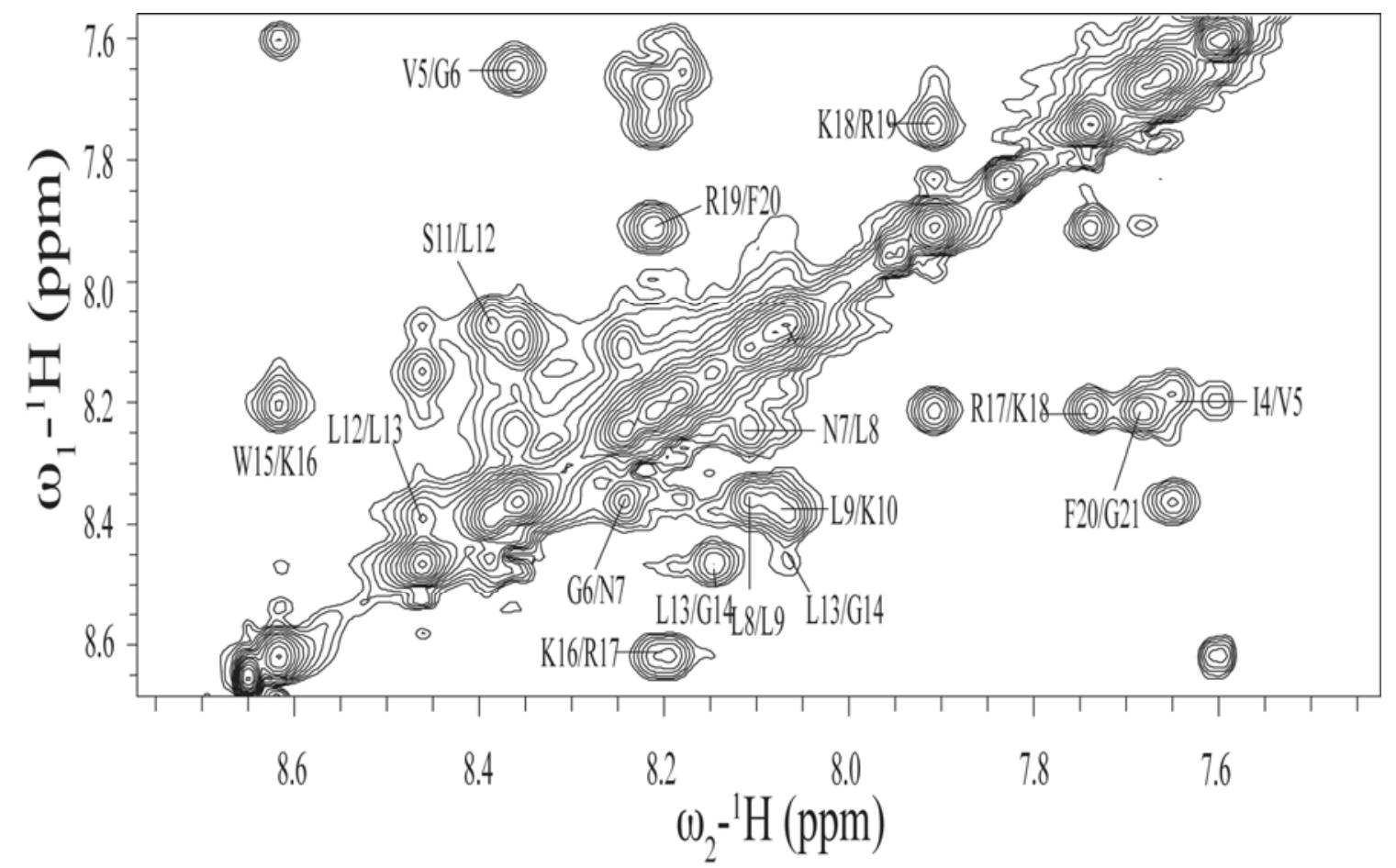

Figure 3.2-18: NH-NH region of two dimensional ${ }^{1} \mathrm{H}-{ }^{1} \mathrm{H}$ NOESY spectrum representing backbone assignments. 
(A)

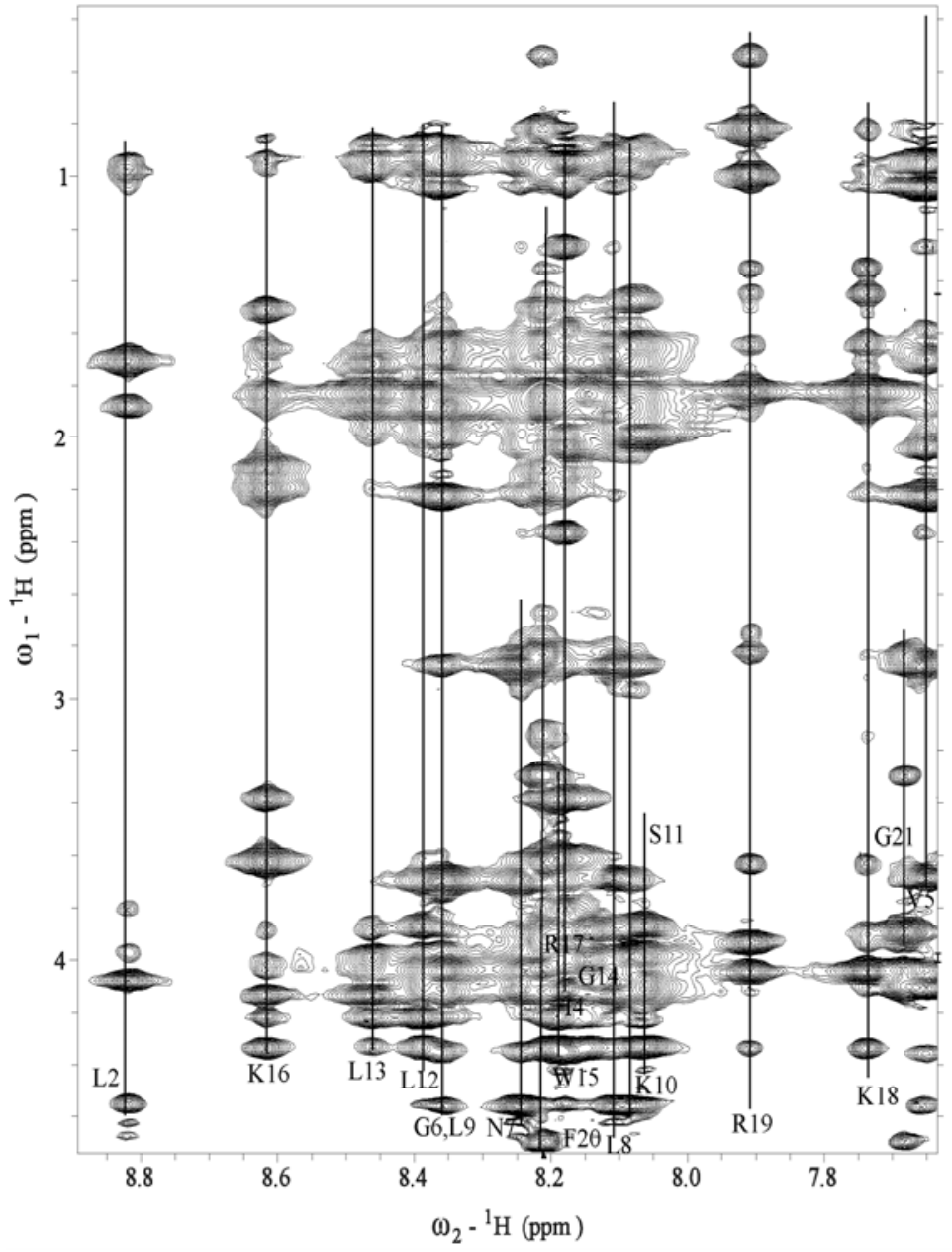

(B)

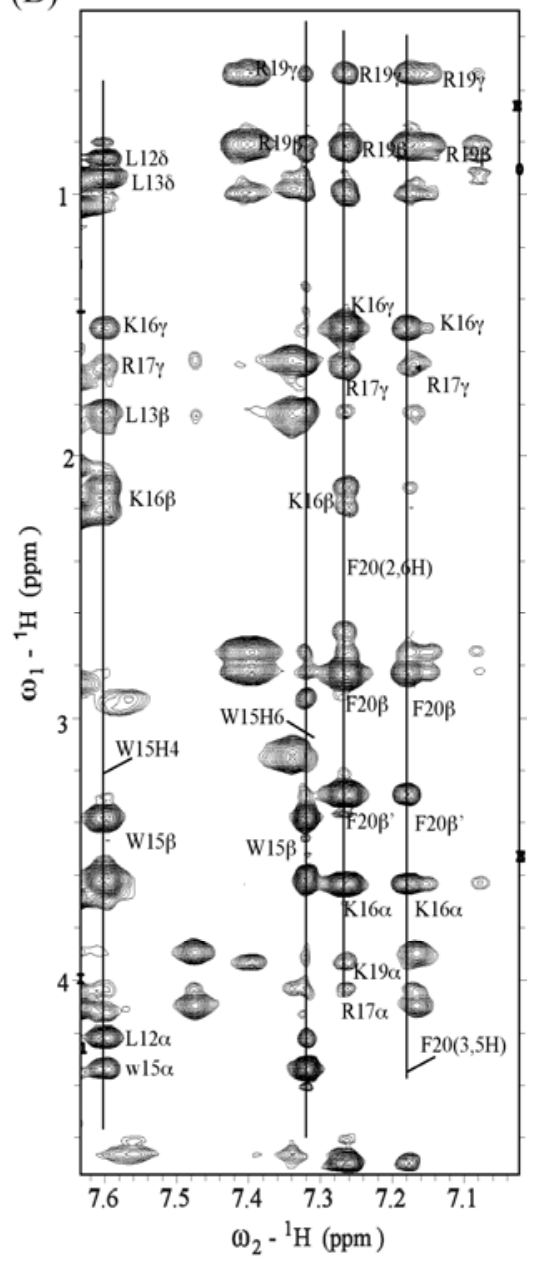

Figure 3.2-19: (A) Resonance assignment of two dimensional ${ }^{1} \mathrm{H}-{ }^{1} \mathrm{H}$ NOESY spectra of LG21 in DPC micelles. (B) Selected region of NOESY spectra showing aliphatic-aromatic side chain interactions.

The two dimensional ${ }^{1} \mathrm{H}-{ }^{1} \mathrm{H}$ NOESY spectra appeared to be well dispersed with large number of intraspecific and interspecific NOEs (Figure 3.2-19, A). In addition of sequential $(i$ to $i+1)$ NOEs, medium range ( $i$ to $i+2, i$ to $i+3$ and $i$ to $i+4)$ NOEs were also noticed for residues from I4 to G21 supporting the presence of helical conformation (Figure 3.2-19, A). The ring protons of W15 and F20 delineate several NOEs with aliphatic aminoacid sidechains (Figure 3.2-19, B). There were side chainside chain interactions between $\mathrm{Leu}^{12}$, Leu ${ }^{13}$ of N-terminal Temporin-1b with $\mathrm{C}$ terminal $\beta$-boomerang $\operatorname{Trp}^{15} \mathrm{H} 4$ and $\operatorname{Trp}^{15} \mathrm{H} 6$. Then there were number of aliphatic- 
aromatic side chain interactions mainly between W15-R19 and K16-F20 (Figure $3.2-19, \mathrm{~B})$.
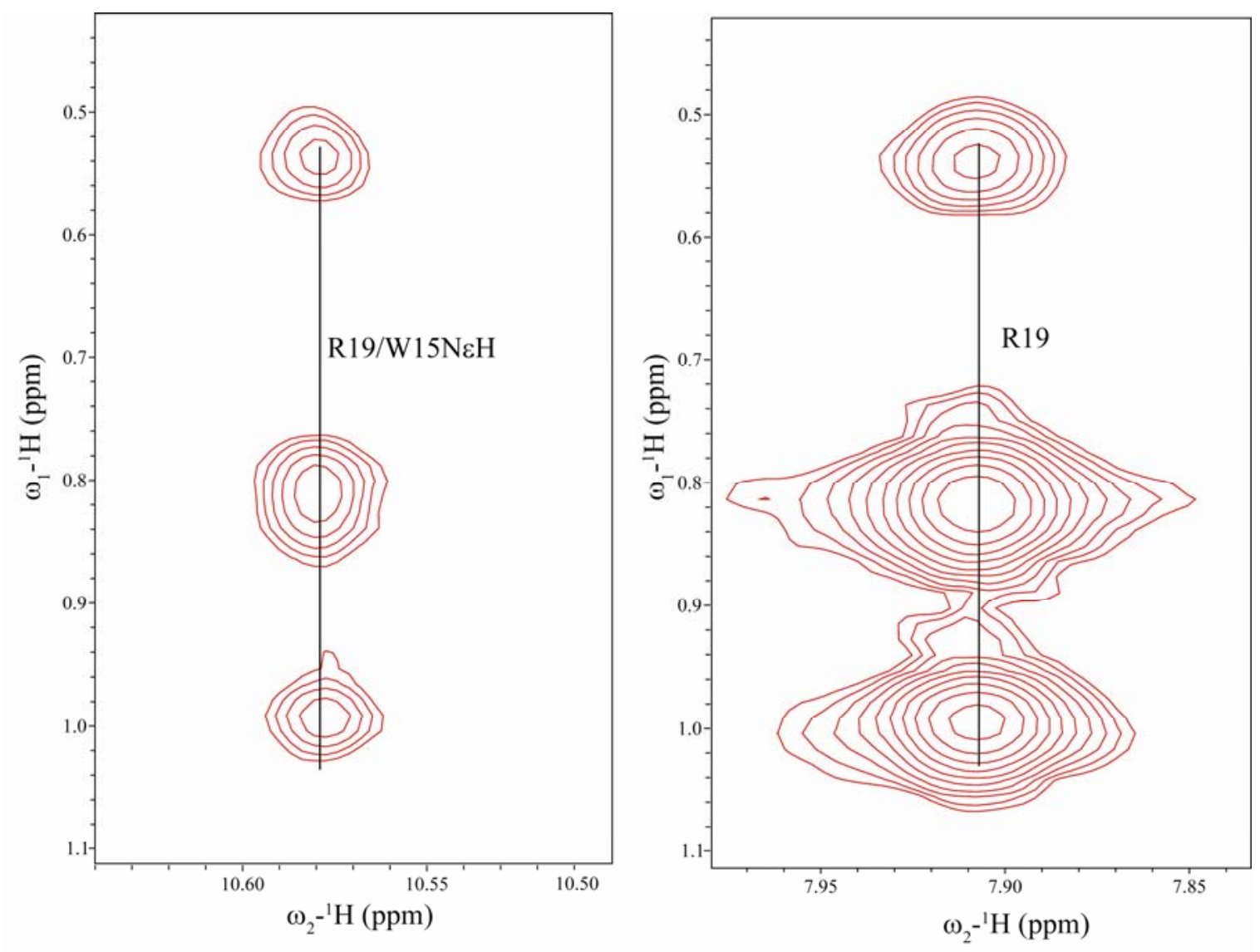

Figure 3.2-20: Right side: R19 residue experiencing abnormal upward chemical shift resonance of its beta and gamma proton. (Left side) Corresponding side chain interaction of R19 aliphatic side chains with indole ring protons of W15.

A remarkable chemical shift of side chain protons of R19 was observed which was shifted upfield (Figure 3.2-20). Such upfield shift of protons is characteristic feature of diamagnetic anisotropy shielding arising from the $\pi$ electron cloud of W15. The $\pi$ electrons in indole ring of Trp gives rise to negatively charged cloud that attracts the cationic side chains of Arg or Lys. Arg is mostly preferred for cation- $\pi$ interaction because of the strong hydrogen bonding character of its guanidium side chains [168]. During cation- $\pi$ interaction, the positively charged side chains of Arg are effectively shielded with negatively charged $\pi$ electrons. Hence apart from its participation in electrostatic attraction of the peptide towards membrane, the R19 residue also 
participates in hydrophobic interactions because of its energetically favourable cation$\pi$ interaction.

${ }^{13} \mathrm{C}^{\alpha}$ spectrum was then assigned with the help of proton chemical shifts. Chemical shift deviations of ${ }^{13} \mathrm{C}^{\alpha}$. and $\mathrm{C}^{\alpha} \mathrm{H}$ from random coil values taken from ref. [169] are used as clue to determine the secondary structure adopted by the peptides [100]. Positive deviation of ${ }^{13} \mathrm{C}^{\alpha}$ chemical shifts and negative deviation of ${ }^{1} \mathrm{H}^{\alpha}$ indicates that the peptide adopts alpha helical conformation.

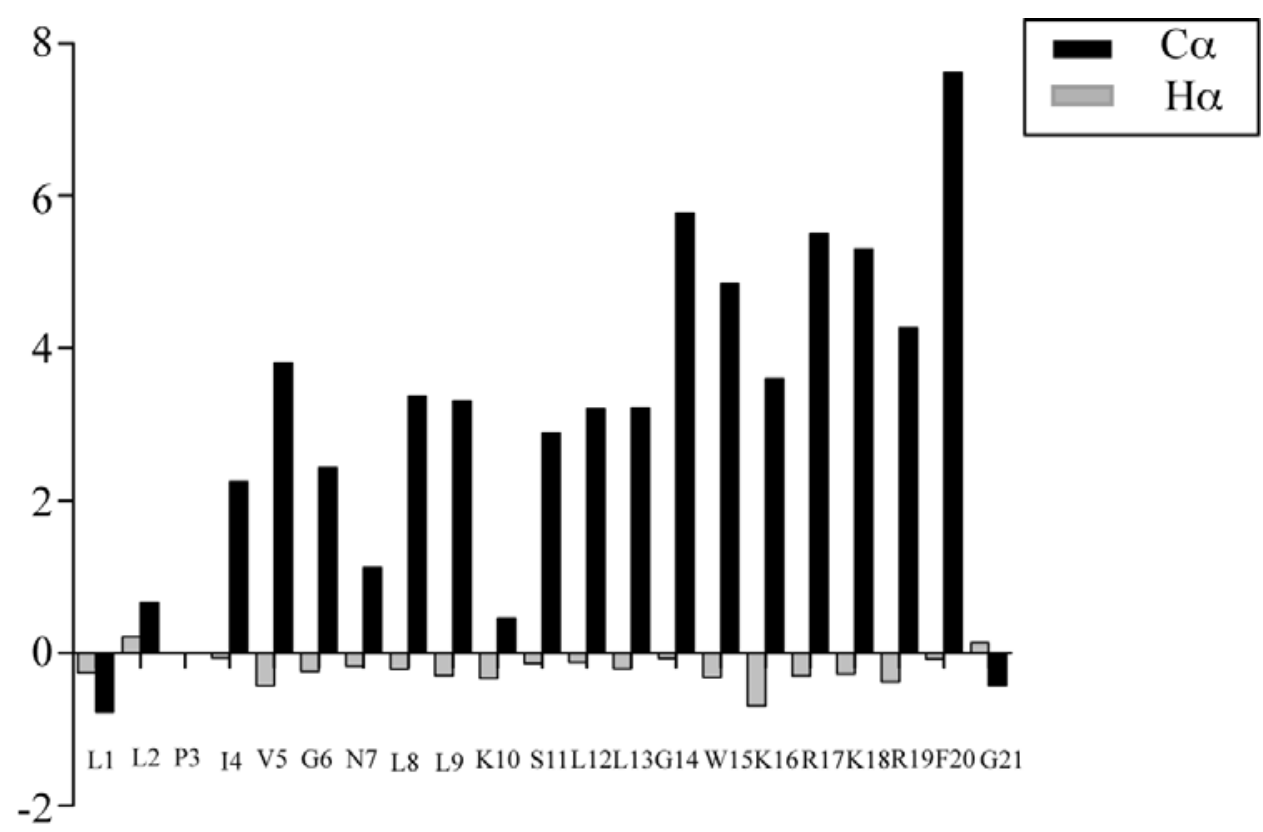

Figure 3.2-21: Difference of observed chemical shift deviations of ${ }^{13} \mathrm{C}^{\alpha}$ and ${ }^{1} \mathrm{H}^{\alpha}$ from random coil values.

The chemical shift deviations also confirmed the presence of alpha helical conformation from I4-F20 (Figure 3.2-21).

\subsubsection{Solution structure of LG21 in DPC micelles:}

Careful analysis of two dimensional ${ }^{1} \mathrm{H}-{ }^{1} \mathrm{H}$ NOESY spectra revealed sequential and medium range NOEs that are categorized as strong, medium and weak peaks. All of these informations were then converted into distance constraints to be used in 
structural calculations using CYANA program. The chemical shifts obtained from ${ }^{1} \mathrm{H}$ and ${ }^{13} \mathrm{C}$-HSQC spectra were then converted into angular constraints using PREDITOR software [170]. By adjusting the violations of distance constraints, several rounds of structural calculations were performed. Out of 100 structures calculated, best 20 structures that show lowest energy were used for further analysis.

Table 3.2-13: Table summarizing structural statistics of 20 lowest energy structures of LG21 in DPC micelles.

\begin{tabular}{|c|c|}
\hline & LG21 \\
\hline $\begin{array}{l}\text { Distance restraints } \\
\text { intraresidue }(|i-j|=0) \\
\text { sequential } \quad(|i-j|=1) \\
\text { medium range }(2 \leq|i-j| \leq 4) \\
\text { total NOE constraints }\end{array}$ & \begin{tabular}{|l}
36 \\
56 \\
66 \\
158
\end{tabular} \\
\hline $\begin{array}{l}\text { Distance restraints violations } \\
\text { Average NOE violation }(\AA) \\
\text { Maximum NOE violation }(\AA)\end{array}$ & $\begin{array}{l}\leq 0.12 \\
\leq 0.24\end{array}$ \\
\hline $\begin{array}{l}\text { Deviation from mean structure } \\
\text { backbone atoms }\left(\mathrm{N}, \mathrm{C} \alpha, \mathrm{C}^{\prime}\right)(\AA) \\
\text { heavy atoms }(\AA)\end{array}$ & \begin{tabular}{|l|}
0.17 \\
0.69
\end{tabular} \\
\hline $\begin{array}{l}\text { Ramachandran plot for the mean } \\
\text { structure } \\
\% \text { residues in the most favourable and } \\
\text { additionally allowed regions } \\
\% \text { residues in the generously allowed } \\
\text { region } \\
\% \text { residues in the disallowed region. }\end{array}$ & $\begin{array}{l}100 \\
0\end{array}$ \\
\hline
\end{tabular}


(A)

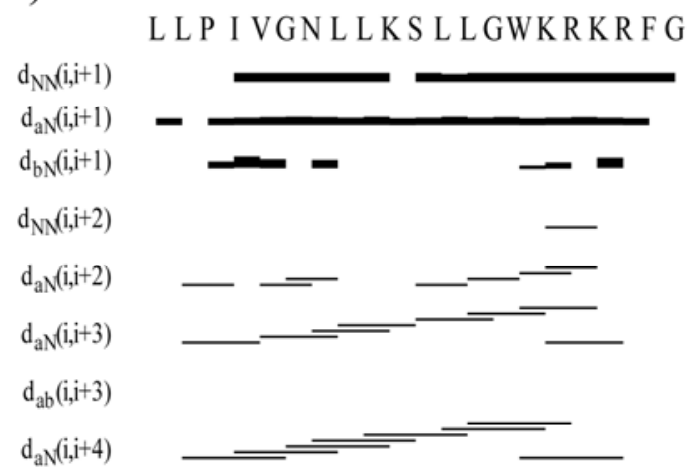

(B)

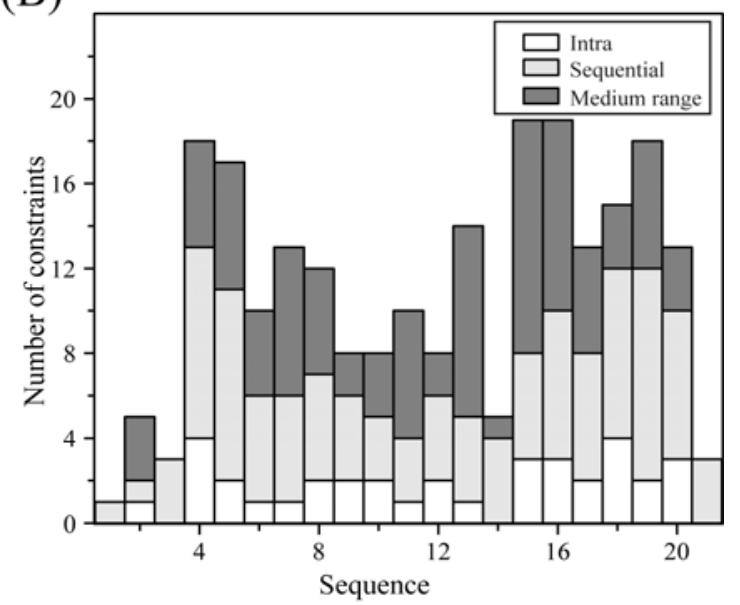

Figure 3.2-22: (A) Bar diagram representing NOE summary. $d_{\alpha N}(i, i+4)$ bars supporting the presence of helical conformation. (B) Bar diagram representing medium range NOE connectivities from I4 to F20 and corresponding number of NOEs.

Structural analysis of LG21 in DPC micelles revealed identification of sequential ( $i$ to i+1) NOEs for all the residues except K10-S11 (Figure 3.2-22, A). Since the peaks were closely overlapping, it was difficult to unambiguously assign the connectivity. Medium range $(i$ to $i+4)$ NOEs that support well ordered alpha helical conformation was also observed for most of the residues (Figure 3.2-22, B). An ensemble of backbone 20 lowest energy structures is shown in Figure 3.2-23, A. The root mean square deviation of heavy and backbone atoms are $0.69 \AA$ and $0.17 \AA$ respectively (Table 3.2-13). The validation of structural data was obtained from Ramachandran plot analysis run by Procheck [171] program. 100\% of the calculated structures were found to be in most favourable and additionally allowed regions (Table 3.2-13).

Single model representation highlighting alpha helical conformation of DPC bound LG21 was presented in Figure 3.2-23, B. The side chain orientations of W15, K16, R19 and F20 are well defined explaining a plausible mode of cation- $\pi$ interaction. The side chains of leucines L8, L9 and L12 also oriented on the same side adding more hydrophobicity. 
(A)

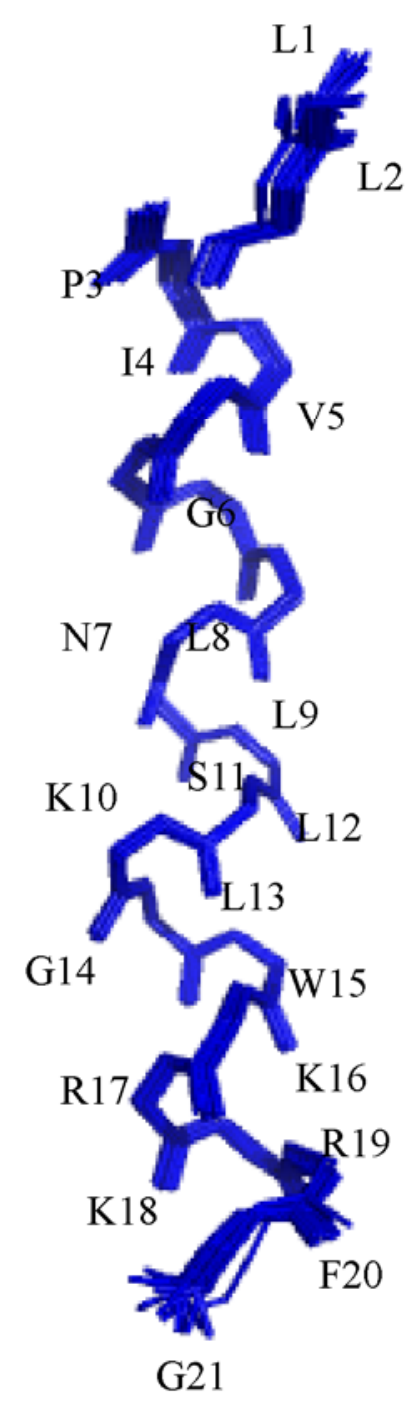

(B)

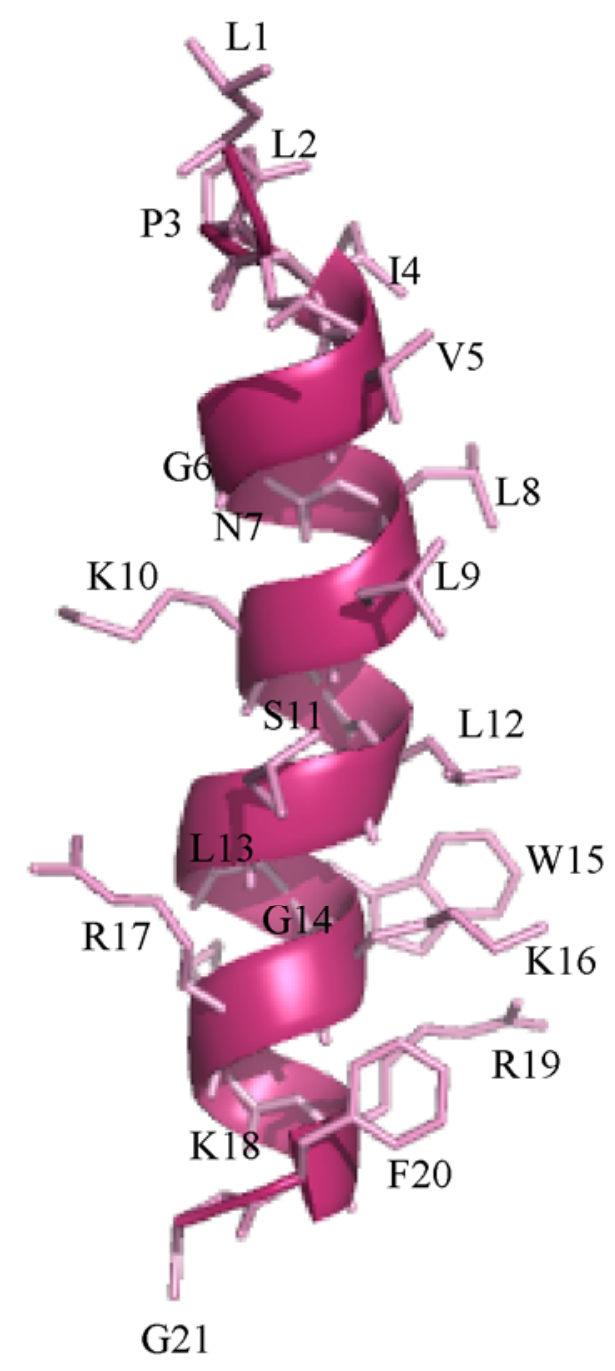

G21

Figure 3.2-23: (A) Superimposition of backbone of 20 lowest energy structures and (B) Single model alpha helical representation of LG21 in DPC micelles.

Surface potential representation of DPC bound LG21 was generated by PYMOL software. The electrostatic potential generated by K16, R17, K18 and R19 as highlighted in blue color in Figure 3.2-24, A helps the peptide to get attracted towards the negatively charged LPS in Gram negative bacteria and LTA in Gram positive bacteria. Once they are attracted, the threshold hydrophobic side chains of L8, L9, 
L12, W15-R19 cation- $\pi$, K16 and F20 (red color in Figure 3.2-24, B) help the peptide to disrupt the outer and inner membrane completely.

(A)

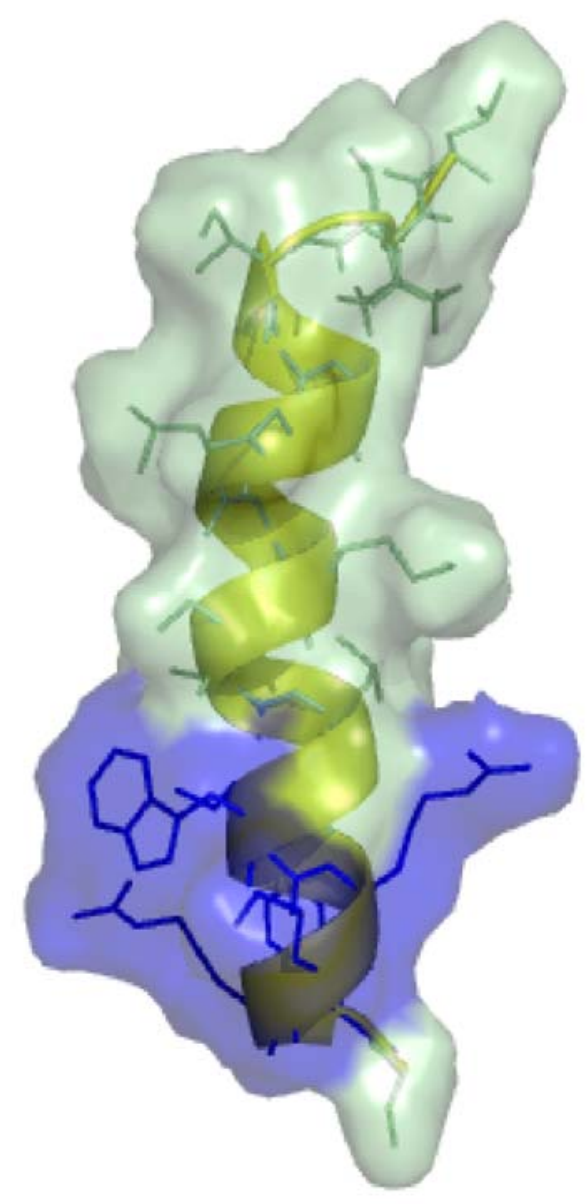

(B)

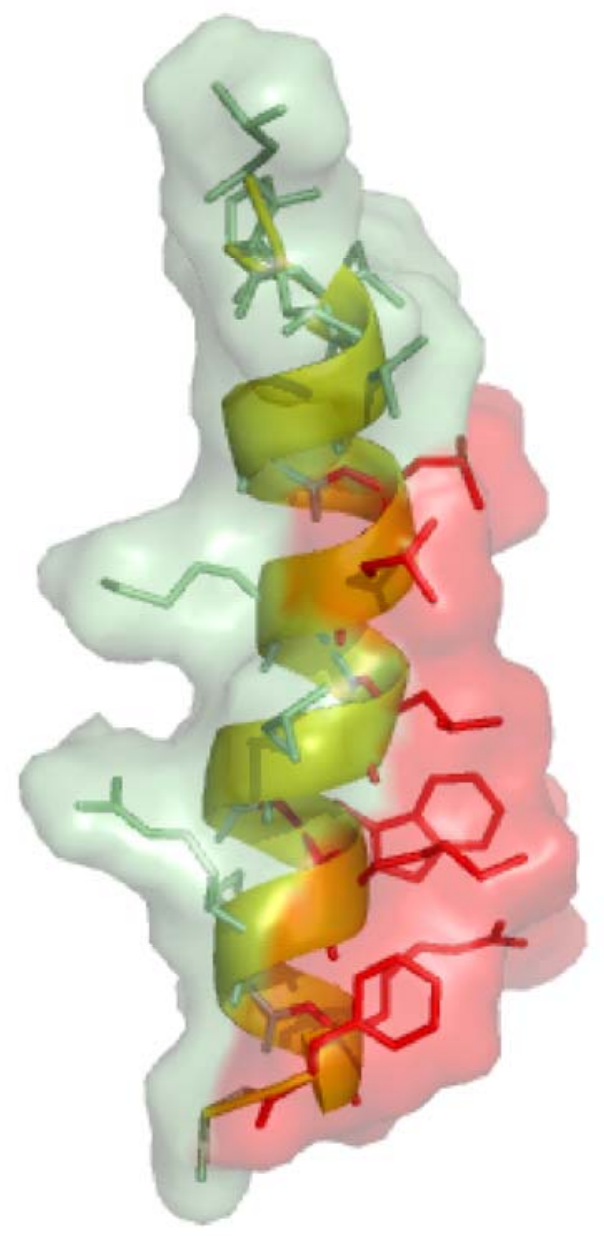

Figure 3.2-24: (A) Surface presentation of LG21 in DPC micelles representing the electrostatic surface (blue color) that is initially required by the peptide to get attracted towards LPS layer and (B) Another model of surface representation of LG21 in DPC micelles highlighting the hydrophobicity (red color) generated by Leucine residues and Cation- $\pi$ interaction.

\subsubsection{NMR spectroscopy of LG21R19A in DPC micelles:}

In order to ascertain the importance of cation- $\pi$ interaction between $\mathrm{W} 15$ and R19, two dimensional ${ }^{1} \mathrm{H}-{ }^{1} \mathrm{H}$ NOESY and ${ }^{1} \mathrm{H}-{ }^{1} \mathrm{H}$ TOCSY spectra were obtained for Ala 
mutant LG21R19A peptide in DPC micelle. Unlike LG21, the ${ }^{1} \mathrm{H}-{ }^{1} \mathrm{H}$ NOESY spectrum of LG21R19A was very broad owing to its weak binding. Only 14 residues out of 21 could be unambiguously assigned (Figure 3.2-25, A). The aliphatic-aromatic side chain interactions were also very weak and limited to few NOEs only (Figure 3.2-25, B). Hence the DPC bound structure of LG21R19A could not be calculated.
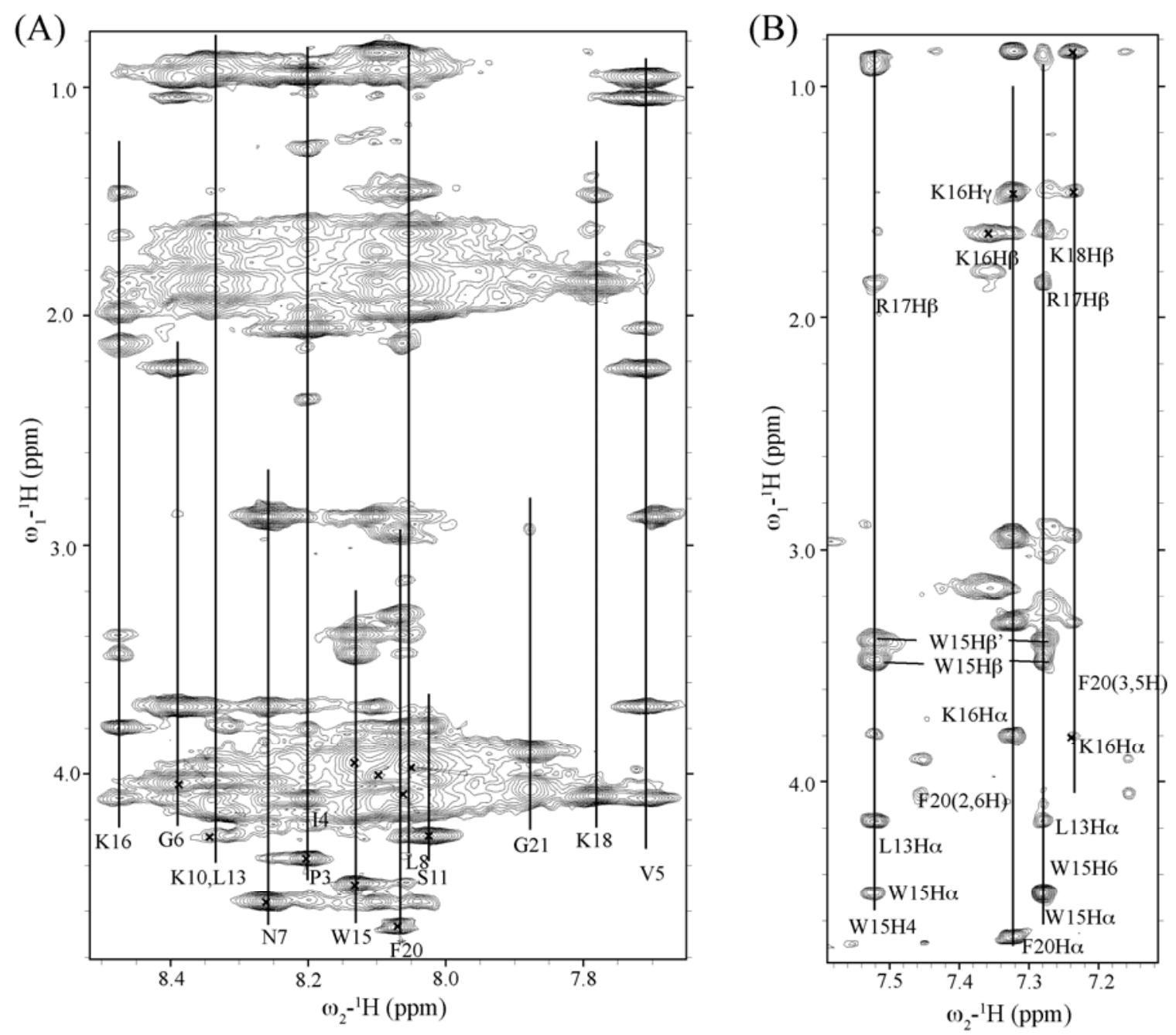

Figure 3.2-25: (A) Resonance assignment of two dimensional ${ }^{1} \mathrm{H}-{ }^{1} \mathrm{H}$ NOESY spectra of LG21R19A in DPC micelles. (B) Selected region of NOESY spectra showing weak aliphatic-aromatic side chain interactions. 


\subsubsection{Discussion:}

Lipopolysaccharides (LPS) present in the outer layer of outer membrane of Gram negative bacteria serves as membrane impermeable barrier for most of the hydrophobic compounds including antimicrobial peptides and antibiotics [172]. Conjugation of two antimicrobial peptides whose activities complement each other had offered a new direction in the development of antiendotoxic antimicrobial peptides. In this present work, the naturally occurring antimicrobial peptides that were known to aggregate on LPS was chosen to conjugate with structured LPS binding motif which is denovo designed (Figure 3.2-26). Structure-Activity Relationship (SAR) studies of conjugated hybrid peptides reveal that such combination of peptides may open up a novel platform in the development of antiendotoxic antimicrobial peptides.

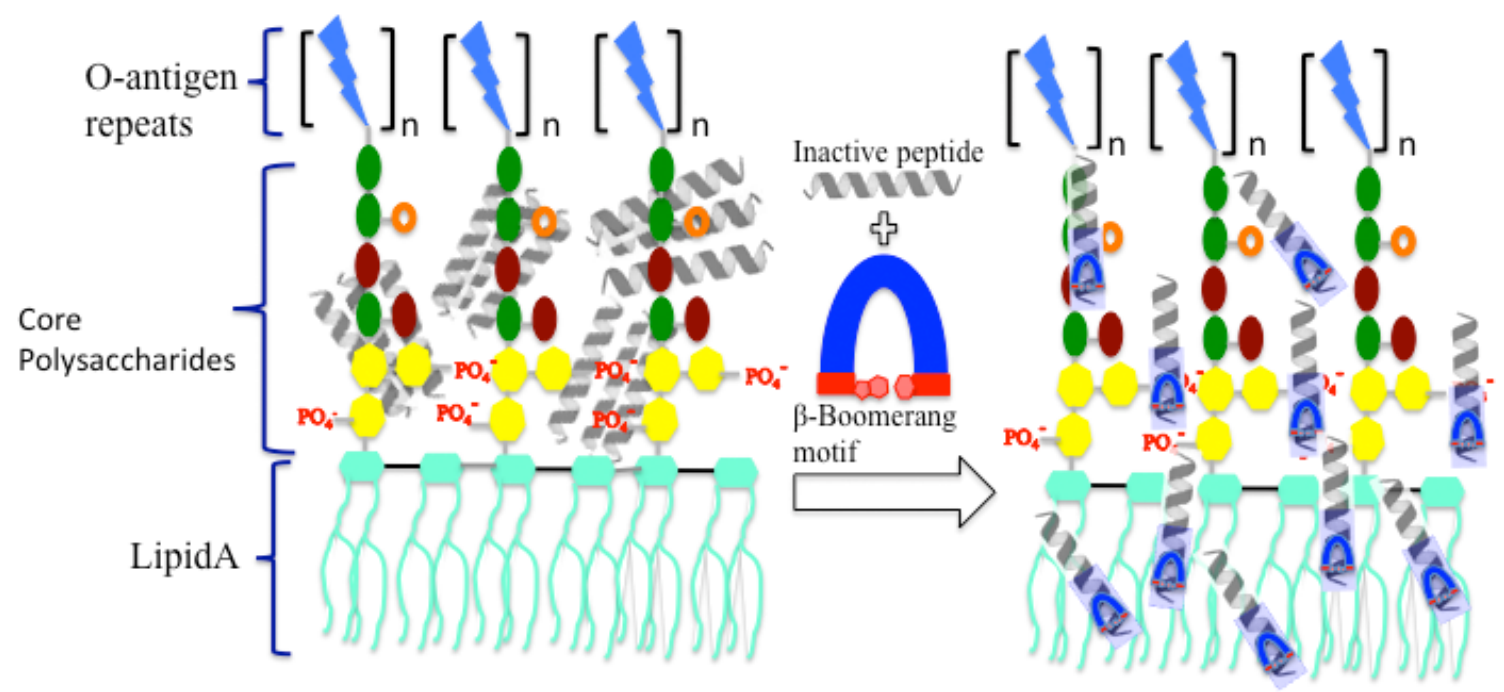

Figure 3.2-26: Model explaining the rationale behind the synthesis of hybrid peptides.

Temporin A, Temporin B and K5L7 peptides were found to undergo oligomerization in the outer layer of outer membrane of Gram negative bacteria. Such big aggregates become impermeable through the outer membrane and hence pronounced inactive. With the conjugation of $\beta$-boomerang motif (highlighted as LPS magnet), these peptides overcome aggregation problem, traverse through the outer and inner membranes and kill bacteria (Figure 3.2-26). 
MIC determination assays clearly demonstrated that the conjugation is very much selective for those peptides that undergo self association on LPS layer of Gram negative bacteria. LG21, FG21 and KG20 demonstrated excellent antibacterial activity.

Alanine mutation of cationic residues in boomerang motif of LG21 revealed an inactive analog LG21R19A. Mutation of polar and cationic residue in temporin part of LG21 does not seem to affect the antibacterial activity. The global conformations of all of these peptides in negatively charged LPS, SDS micelles and neutral DPC micelles reveal alpha helical structures. NMR screening of LG21, FG21, KG20 and LG21R19A was done in LPS, SDS and DPC micelles. Owing to the tight binding of these peptides with LPS in the sub micromolar range of $0.6 \mu \mathrm{M}, 0.6 \mu \mathrm{M}$ and $0.3 \mu \mathrm{M}$, no tr-NOESY effect was observed. Only LG21 with DPC micelles yielded a well dispersed and analyzable spectrum.

Atomic resolution structure of LG21 supports the alpha helical conformation and also provides the reason behind the inactiveness of LG21R19A peptide. The alpha helical conformation brings $\mathrm{W} 15$ and R19 residues close together that they engage in cation $\pi$ interaction. This type of interaction is widely seen in peptides rich in Arg and $\operatorname{Trp}$ residues. Apart from the bulk hydrophobicity that is created with side chains of Trp, the indole ring of $\operatorname{Trp}$ exposes intense $\pi$-electron cloud that attracts the positively charged guadinium side chains of Arg. Moreover, Arg can form hydrogen bonds with surrounding water molecules while involved in cation $-\pi$ interaction also [168].

The cationic residues in boomerang motif of LG21 can initially get attracted towards the membrane by electrostatic attraction. The threshold hydrophobicity needed to disrupt the outer membrane could then be achieved primarily with the cation- $\pi$ between W15 and R19 followed by leucine L8, L9, L12 side chains. Segregation of leucine on one side forming leucine hydrophobic patch was also achieved with this conjugation of peptides. Such leucine patch mediated antimicrobial activity has also been noted leu rich DFTamp1 [173]. Hence a single mutation of R19 to A19 has caused drastic change leading to complete destruction of activity.

All of these hybrid peptides were also found not to self associate in the presence of LPS as in temporins and K5L7 peptide. Whereas LG21R19A seem to undergo self 
association. The outer membrane permeability assays with fluorescent probes NPN, SYTOX green and membrane depolarization assays also revealed that the importance of such cation- $\pi$ interaction whereby LG21R19A remained largely inactive. At MIC concentrations, these peptides were not found to be hemolytic and can neutralize LPS also effectively.

Collectively these results suggest the importance of boomerang motif in conjugation of peptides that undergo self association in the LPS layer. This opens up the novel platform the design of antimicrobial peptides with antiendotoxic property. 


\subsection{CYSTEINE DELETED ANALOGS OF PROTEGRINS}

\subsubsection{Peptide design:}

Ever since the discovery of antimicrobial peptides that are ribosomally synthesized in all form of vertebrates in different kinds of secretions, they are successfully used as template for the generation of highly active broad spectrum antibiotics. Structurally these antimicrobial peptides can be categorized in to alpha helix, beta hairpins or extended conformations. Among the beta hairpins that are extensively studied, Protegrin family of AMPs occupies a prominent position. Protegrins are a group of antimicrobial peptides that are identified in porcine leucocytes [174]. Their broad spectrum of activity and aminoacid distribution were found to be a blend of defensins and tachyplesins, antimicrobial peptides found in polymorpho neutrophils and horse shoe crab hemocytes respectively. [174].

Protegrin PG-1 is composed of 18 aminoacid residues with four cysteine forming two disulfide linkages between $\mathrm{C} 6-\mathrm{C} 15$ and $\mathrm{C} 8-\mathrm{C} 13$ (Table 3.3-1). Hence it adopts antiparallel beta hairpin conformation with apolar residues accumulated in the center and charged polar residues confined to the turn and tails [175].

Table 3.3-1: Peptides designed based on Protegrin PG-1 and their aminoacid sequences.

\begin{tabular}{|l|l|}
\hline Peptide & Primary structure \\
\hline PG-1 & R-G-G-R-L-C-Y-C-R-R-R-F-C-V-C-V-G-R \\
\hline RR14 & R-G-G-R-L-Y-R-R-R-F-V-V-G-R \\
\hline RR11 & R-L-Y-R-R-R-F-V-V-G-R \\
\hline LR10 & L-Y-R-R-R-F-V-V-G-R \\
\hline
\end{tabular}

Protegrin PG-1 is found to have similar characteristics with tachyplesin except the distribution of polar and apolar residues. Cystiene deleted analog of tachyplesin (CDT) was found to adopt the $\beta$-hairpin like conformation even in the absence of disulfide bonds [32]. CDT was found to retain antimicrobial activity and LPS binding 
nature with increase in toxicity to mammalian cells [33]. Also most membrane active peptides are alpha helical and those that fall under beta category have Cys in their sequences. So, in this present study, cysteine deleted analogs of PG-1, RR14 was designed and structure-activity characterization was performed (Table 3.3-1).

Molecular dynamic simulation studies of PG-1 in SDS and DPC micelles suggest that the one strand of PG-1 (RGGRLCYCRR) is responsible of antimicrobial activity and the other strand (RFCVCVGR) is responsible for toxicity [176]. It was also found that the first four residues RGGR is very much flexible which imparts ample mobility to R1 residue to initiate binding to various membranes [177]. Hence in the present study, along with RR14, full length cysteine deleted PG-1, RR11 - RGG deleted and LR10 RGGR deleted analogs were also studied extensively (Table 3.3-1).

\subsubsection{Activity assays:}

\subsubsection{Antimicrobial activity:}

As an initial step towards structure-activity relationship studies of deleted analogs of PG-1, minimum inhibitory concentration was determined by broth dilution method in representative Gram negative and Gram positive strains. The order of peptides for antibacterial activity is RR14>RR11 $>$ LR10. The full length cysteine deleted analog of PG-1 (RR14) exhibits comparable antibacterial activity to PG-1 as reported in [178] followed by RR11 (Table 3.3-2). RGGR deleted analog, LR10 exhibited no killing activity (Table 3.3-2). This result suggests the importance of RGGR residues in antimicrobial activity. It is also envisioned that the deletion of disulfide bridges does not affect antibacterial activity.

The intramolecular disulfide bonds were found to be essential for PG-1 to be active in physiological salt concentrations [34]. Hence the deleted analogs were tested for antibacterial activity in the presence of $150 \mathrm{mM} \mathrm{NaCl}$ concentration. RR14 peptide was active against Gram positive bacteria whereas it showed slightly higher MIC values in Gram negative bacteria (Table 3.3-2). RR11 and LR10 peptides show diminished or no antibacterial activity at all in the presence of $\mathrm{NaCl}$ (Table 3.3-2). Collectively, MIC results suggest that the RGGR residues are important for antibacterial activity, deletion of which leads to inactive peptide. 
Table 3.3-2: Minimum inhibitory concentration (MIC in $\mathbf{m M}$ ) of deleted analogs of PG-1 in Gram negative and Gram positive bacteria.

\begin{tabular}{|c|c|c|c|c|c|c|}
\hline \multirow[t]{2}{*}{ Bacteria } & \multicolumn{2}{|l|}{ RR14 } & \multicolumn{2}{|l|}{ RR11 } & \multicolumn{2}{|l|}{ LR10 } \\
\hline & MHB & $\begin{array}{l}\mathrm{MHB}^{+} \\
150 \mathrm{mM} \\
\mathrm{NaCl}\end{array}$ & MHB & $\begin{array}{l}\text { MHB+ } \\
150 \mathrm{mM} \\
\mathrm{NaCl}\end{array}$ & MHB & $\begin{array}{l}\text { MHB+ } \\
150 \mathrm{mM} \\
\mathrm{NaCl}\end{array}$ \\
\hline \multicolumn{7}{|l|}{ Gram negative } \\
\hline $\begin{array}{l}\text { E.coli } \\
\text { (lab strain) }\end{array}$ & 4 & 50 & 10 & $>200$ & 50 & $>200$ \\
\hline $\begin{array}{l}\text { P.aeruginosa } \\
\text { ATCC } 27853\end{array}$ & 8 & 25 & 25 & $>200$ & 200 & $>200$ \\
\hline $\begin{array}{l}\text { K.pneumoniae } \\
\text { ATCC } 13883\end{array}$ & 8 & 100 & 25 & $>200$ & $>200$ & $>200$ \\
\hline $\begin{array}{l}\text { S.enterica } \\
\text { ATCC } 14028\end{array}$ & 8 & 25 & 25 & $>200$ & 200 & $>200$ \\
\hline \multicolumn{7}{|l|}{ Gram positive } \\
\hline $\begin{array}{l}\text { B.subtilis } \\
\text { (lab strain) }\end{array}$ & 8 & 12.5 & 10 & $>200$ & 50 & $>200$ \\
\hline $\begin{array}{l}\text { S.aureus } \\
\text { ATCC } 25923\end{array}$ & 2 & 10 & 8 & $>200$ & 25 & $>200$ \\
\hline $\begin{array}{l}\text { S.pyogenes } \\
\text { ATCC } 19615\end{array}$ & 8 & 50 & 25 & $>200$ & 50 & $>200$ \\
\hline $\begin{array}{l}\text { E.faecalis } \\
\text { ATCC } 29212\end{array}$ & 4 & 25 & 25 & $>200$ & 25 & $>200$ \\
\hline
\end{tabular}

\subsubsection{Biophysical characterization:}

\subsubsection{Outer membrane permeability:}

The first and foremost responsibility of antimicrobial peptides is to trespass the outer membrane in order to kill the bacterial cells. Hence the outer membrane permeability 
of the deleted analogs of PG-1 was analysed using a hydrophobic dye NPN (1-Nphenylnapthylamine).

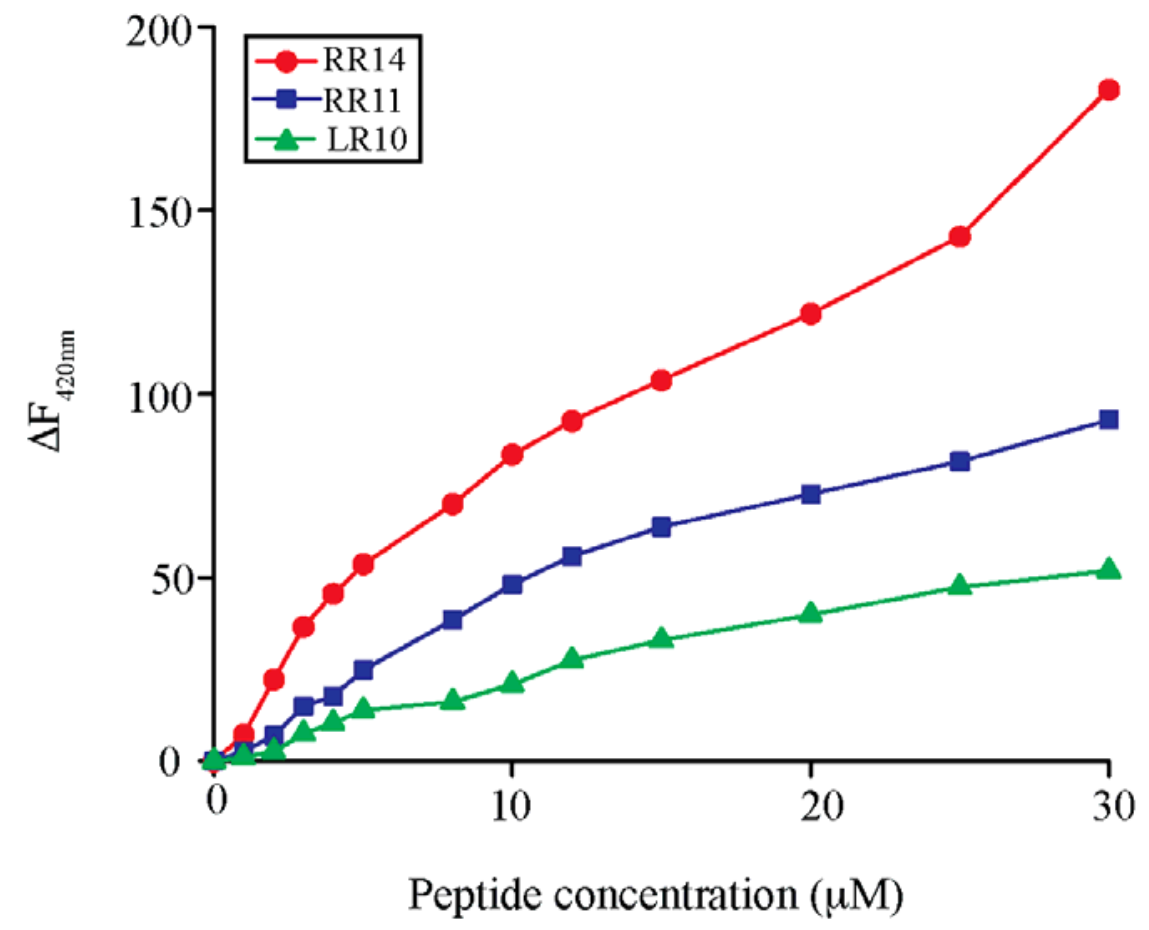

Figure 3.3-1: Outer membrane permeability of deleted analogs of PG-1 in E.coli cells. $\Delta \mathbf{F}_{420 \mathrm{~nm}}$ is the difference between fluorescence intensities after and before addition of peptides at emission max of NPN at $420 \mathrm{~nm}$.

In parallel with the MIC values (Table 3.3-2), RR14 showed higher permeability followed by RR11 and LR10 (Figure 3.3-1). The increase in fluorescence intensity was dose dependent for all of the peptides tested.

\subsubsection{Outer and inner membrane permeability:}

In order to check the ability of the peptides to completely disrupt the cells, E.coli cells were treated with increasing concentrations of peptides in the presence of SYTOX green dye. This dye fluoresces only when it binds to intracellular nucleic acids. When the intact E.coli cells were treated with increasing concentrations of peptides, there were different grades of increase in fluorescent intensity indicating distinct ability of the peptides to internalize the membrane permeable dye. Unlike outer membrane permeability, RR14 peptide demonstrates considerably greater membrane disruption 
marked by its distinct increase in $\Delta \mathrm{F}_{420 \mathrm{~nm}}$ values (Figure 3.3-2). RR11 and LR10 peptides show very weak ability to completely disrupt the cells (Figure 3.3-2).

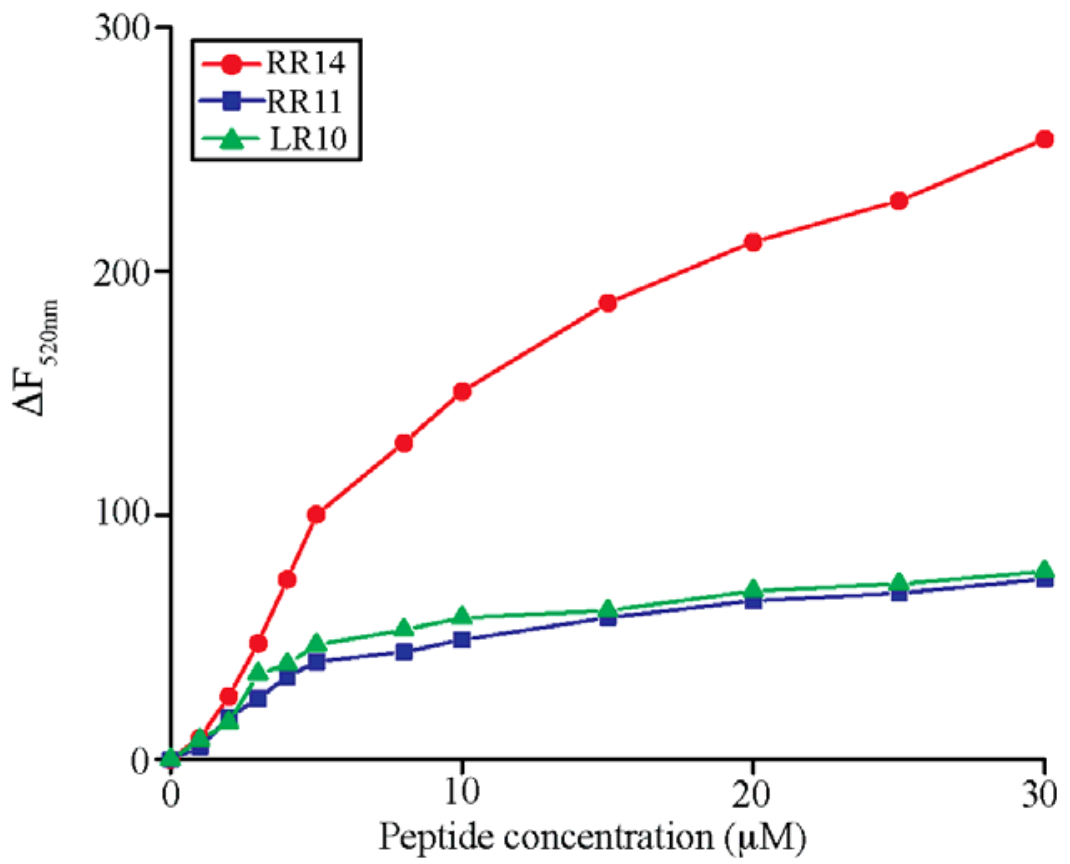

Figure 3.3-2: Complete membrane disrtuption by deleted analogs of PG-1 in E.coli cells. $\Delta \mathbf{F}_{420 \mathrm{~nm}}$ is the difference between fluorescence intensities after and before addition of peptides at emission max of SYTOX green at $520 \mathrm{~nm}$.

\subsubsection{Membrane permeability invitro:}

Liposomes are used widely to mimic the membrane ambience and calcein entrapped in POPC: LPS liposomes are used to investigate membrane lysis by the peptides. Compromising with the permeability assays in intact E.coli cells, RR14 demonstrates higher leakage when compared to other peptides (Figure 3.3-3). At about $10 \mu \mathrm{M}$ of peptide concentration itself, it exhibits $50 \%$ leakage followed by steady increase reaching $80 \%$ of lysis at $30 \mu \mathrm{M}$ concentration. On the other hand, RR11 shows $30 \%$ of leakage and LR10 shows only 10\% leakage (Figure 3.3-3) at the highest peptide concentration tested. 


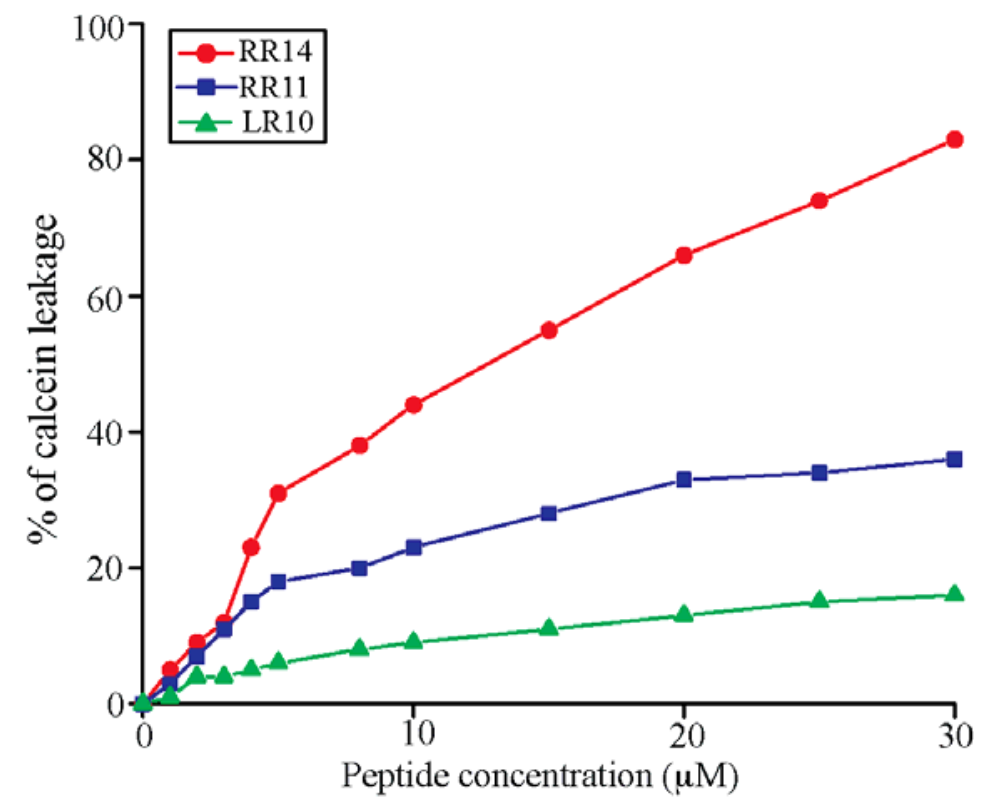

Figure 3.3-3: Membrane permeabilization invitro by deleted analogs of PG-1 in POPC: LPS (80:20 w/v) liposomes.

\subsubsection{Dissociaiton of FITC-LPS aggregates:}

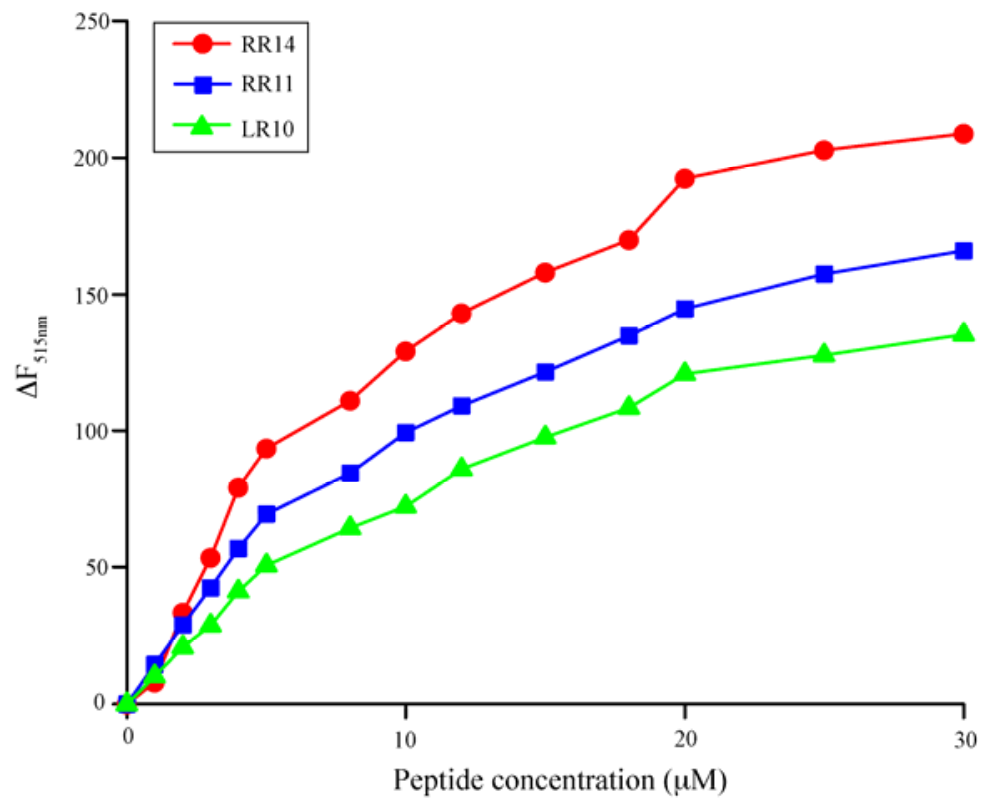

Figure 3.3-4: Dissociation of FITC conjugated LPS aggregates by deleted analogs of PG-1. $\Delta \mathrm{F}_{515 \mathrm{~nm}}$ is the difference between fluorescence intensities after and before addition of peptides at emission max of FITC at $515 \mathrm{~nm}$. 
The distinctive ability of the peptides to dissociate LPS aggregates that correlate with membrane permeability assays were determined using FITC conjugated LPS. FITC experiences self quenching when entrapped inside LPS micelles, dequenching of which demonstrates dissociation of LPS micelles.

The differential ability of the peptides to perturb LPS aggregates decreases with sequential deletion of Arg residues with RR14 showing highest perturbing ability followed by RR11 and LR10 (Figure 3.3-4). Hence the importance of initial electrostatic attraction mediated by Arg residues in N-terminal of PG-1 is justified.

\subsubsection{Isothermal titration calorimetry:}
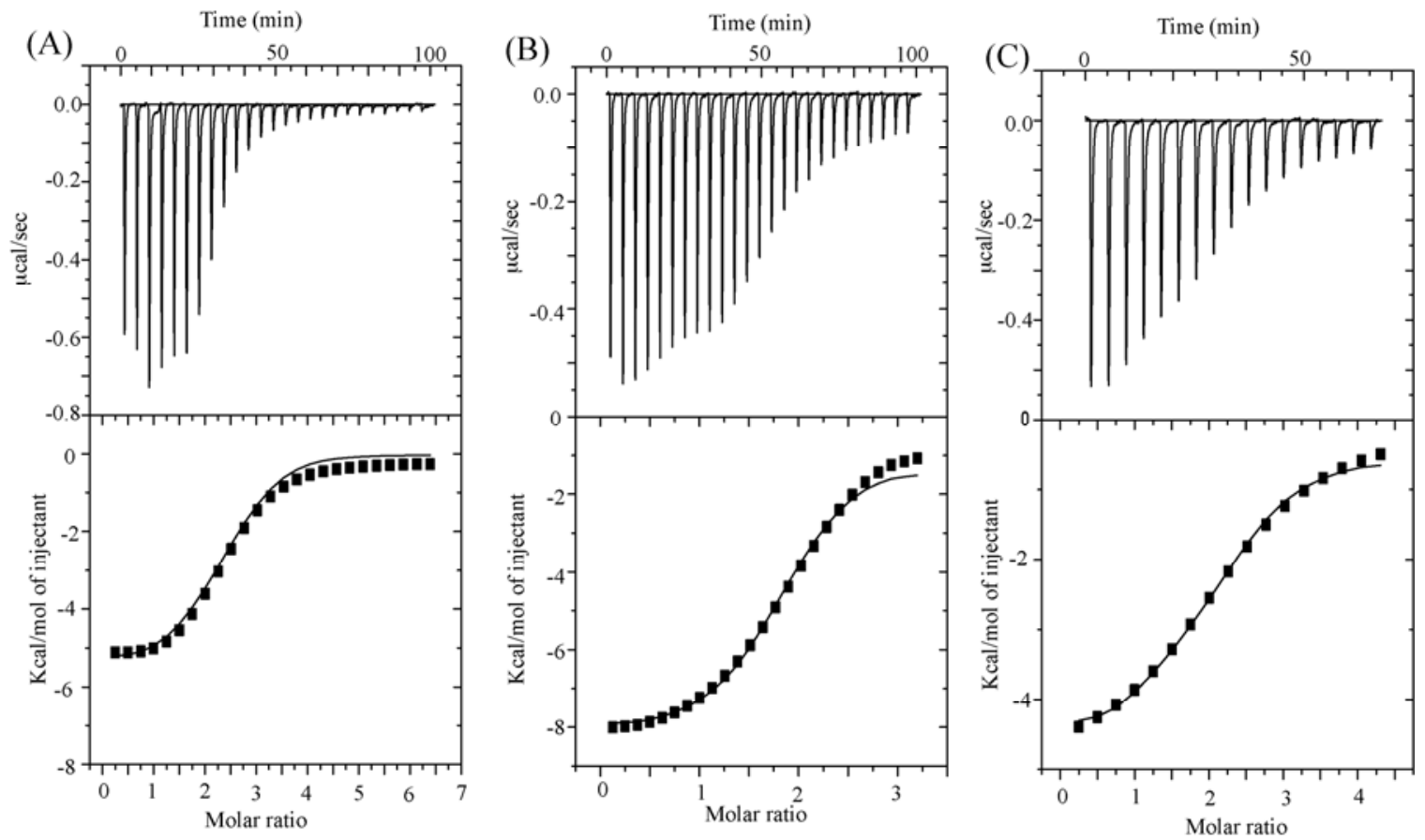

Figure 3.3-5: Thermondynamic binding profiles of cysteine deleted analogs of PG-1 in LPS micelles at $25^{\circ} \mathrm{C}$.

The thermodynamic principles that govern the binding to peptides to LPS micelles were analysed by ITC experiments. Peptides from stock of higher concentrations (1 $\mathrm{mM}$ ) were injected into LPS micelles at $25^{\circ} \mathrm{C}$. The binding of peptides to LPS was found to be exothermic in nature as revealed by peaks facing downwards and enthalpy driven (Figure 3.3-5). Because of the presence of more cationic residues, electrostatic 
interactions play predominant role in binding. Irrespective of activity, all the deleted analogs were found to bind with LPS. But the dissociation constant, $\mathrm{K}_{d}(\mu \mathrm{M})$ values explains the extent of binding. The more, the $\mathrm{K}_{\mathrm{d}}$ value, the lesser the binding.

Table 3.3-3: Calculation of thermodynamic parameters for the binding of cysteine deleted analogs of PG-1 with LPS micelles.

\begin{tabular}{|l|l|l|l|}
\hline & RR14 & RR11 & LR10 \\
\hline $\mathrm{K}_{\mathrm{a}}\left(\mu \mathrm{M}^{-1}\right)$ & 2.79 & 1.04 & 0.33 \\
\hline$\Delta \mathrm{H}\left(\mathrm{kcal}^{\mathrm{m}} \mathrm{mol}^{-1)}\right.$ & -5.4 & -8.5 & -5.1 \\
\hline $\mathrm{T} \Delta \mathrm{S}\left({\left.\mathrm{kcal} . \mathrm{mol}^{-1} \mathrm{deg}^{-1}\right)}^{-1}\right.$ & 3.3 & -0.29 & 2.4 \\
\hline$\Delta \mathrm{G}\left(\mathrm{kcal} . \mathrm{mol}^{-1}\right)$ & -8.7 & -8.2 & -7.5 \\
\hline $\mathrm{K}_{\mathrm{d}}(\mu \mathrm{M})$ & 0.35 & 0.96 & 3 \\
\hline
\end{tabular}

It was noted that the $\mathrm{K}_{\mathrm{d}}$ value increases with decrease in the length and number of arginine residues (Table 3.3-3). The full length cysteine deleted analog of PG-1, RR14 shows tight binding with $\mathrm{K}_{d}$ value of $0.3 \mu \mathrm{M}$ followed by RR11. RGGR deleted LR10 showed considerably high $\mathrm{K}_{\mathrm{d}}$ value of $3 \mu \mathrm{M}$ (Table 3.3-3).

\subsubsection{Structural characterization:}

\subsubsection{Circular Dichroism spectroscopy:}

The global fold of the peptides in anioinic and zwitterionic micelles were examined using CD spectroscopy. Far UV CD spectra were first obtained for $50 \mu \mathrm{M}$ of peptides RR14, RR11 and LR10 in aqueous $10 \mathrm{mM}$ phosphate buffer to evaluate the conformation of the peptide in free state. All of the peptides displayed minima around $195 \mathrm{~nm}$ that is characteristic of random coil conformations (Figure 3.3-6). In the presence of $30 \mu \mathrm{M}$ of LPS micelles, the peptides, irrespective of activity, exhibited minima around $210 \mathrm{~nm}$ and maxima around $195 \mathrm{~nm}$ that are characteristic of $\beta$-sheet conformation (Figure 3.3-6). Whereas, in the presence of zwitterionic DPC micelles the peptides displayed minima around $195 \mathrm{~nm}$ which is again characteristic of random coil (Figure 3.3-6). The ability of PG-1 to differentiate between mammalian and bacterial cells were seem to be conserved even after the deletion of disulphide bridges. 
(A)

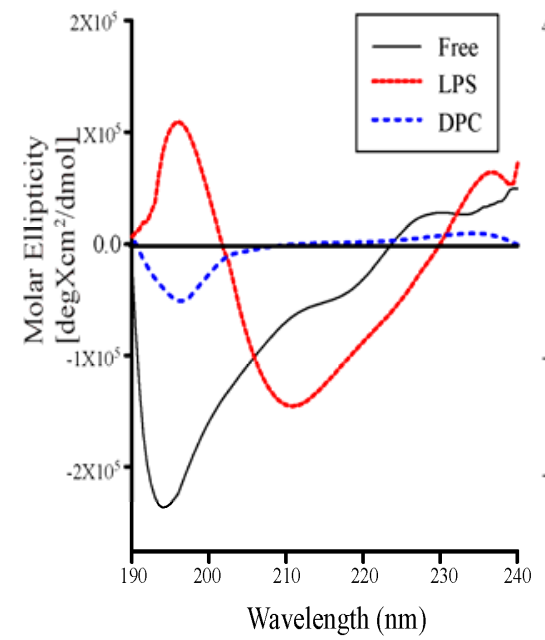

(B)

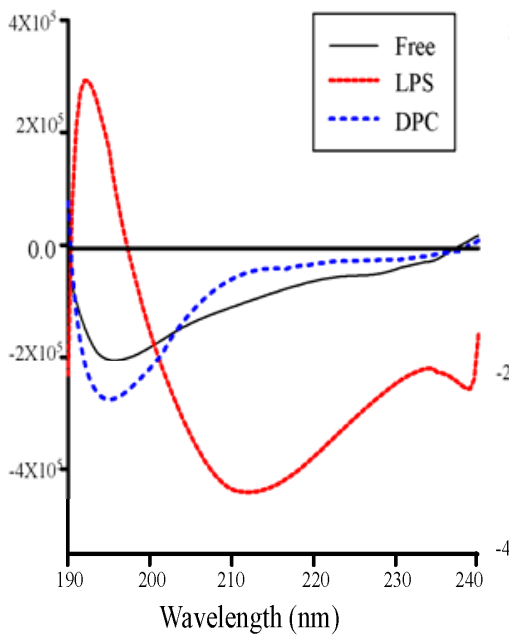

(C)

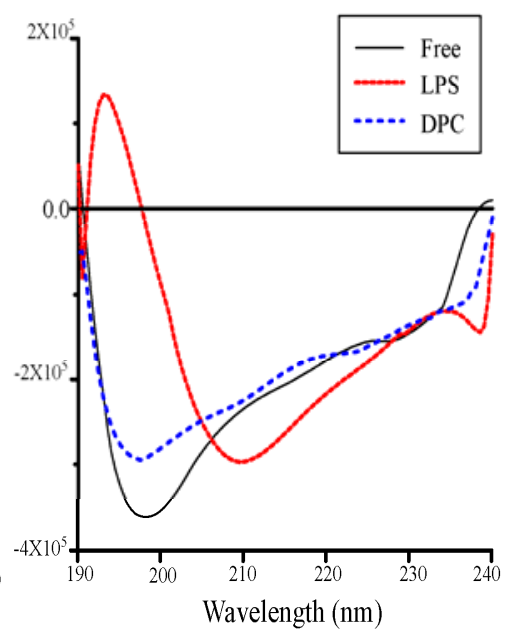

Figure 3.3-6: Far UV CD spectra of $50 \mu \mathrm{M}$ of (A) RR14, (B) RR11 and (C) LR10 in buffer (black line), $30 \mu \mathrm{M}$ LPS (red dotted line) and $20 \mathrm{mM}$ DPC (blue dotted line).

\subsubsection{NMR spectroscopy: Solution NMR and tr-NOESY studies of peptides in LPS micelles:}

Two dimensional ${ }^{1} \mathrm{H}_{-}{ }^{1} \mathrm{H}$ TOCSY and ${ }^{1} \mathrm{H}^{1}{ }^{1} \mathrm{H}$ NOESY spectra of RR14, RR11 and LR10 were obtained in its free state at $298 \mathrm{~K}$ and $\mathrm{pH} 4.5$. The analysis of the spectra revealed only intra residue and sequential NOEs indicating the peptide did not adopt any specific strcuture and in random coil conformation. There was also lack of side chain-side chain interactions. It should be recalled that PG-1 folds into antiparallel beta hairpin structure in free state itself with the help of disulphide bridges.

The peptides RR14, RR11 and LR10 were then titrated with increasing concentrations of LPS from stock of $10 \mathrm{mg} / \mathrm{ml}(1 \mathrm{mM})$ and a series of 1-D spectra were recorded. Substantial measure of broadening could be noticed in the presence of $50 \mu \mathrm{M}$ of LPS and hence two dimensional ${ }^{1} \mathrm{H}-{ }^{1} \mathrm{H}$ TOCSY and ${ }^{1} \mathrm{H}-{ }^{1} \mathrm{H}$ NOESY spectra were recorded at this ratio (1:10 of LPS: peptide).

Since LPS forms larger aggregates in aqueous medium, tr-NOESY has proven to be a versatile tool in elucidating structure of LPS-peptide complexes [150]. Analyses of trNOE spectra revealed intra residue, sequential $(i-i+1)$ and medium range of $(i-i+2)$ 
NOEs. Sequence specific resonance assignment of all of the residues was achieved for RR14 and RR11 in the presence of LPS micelles (Figure 3.3-7).

(A)

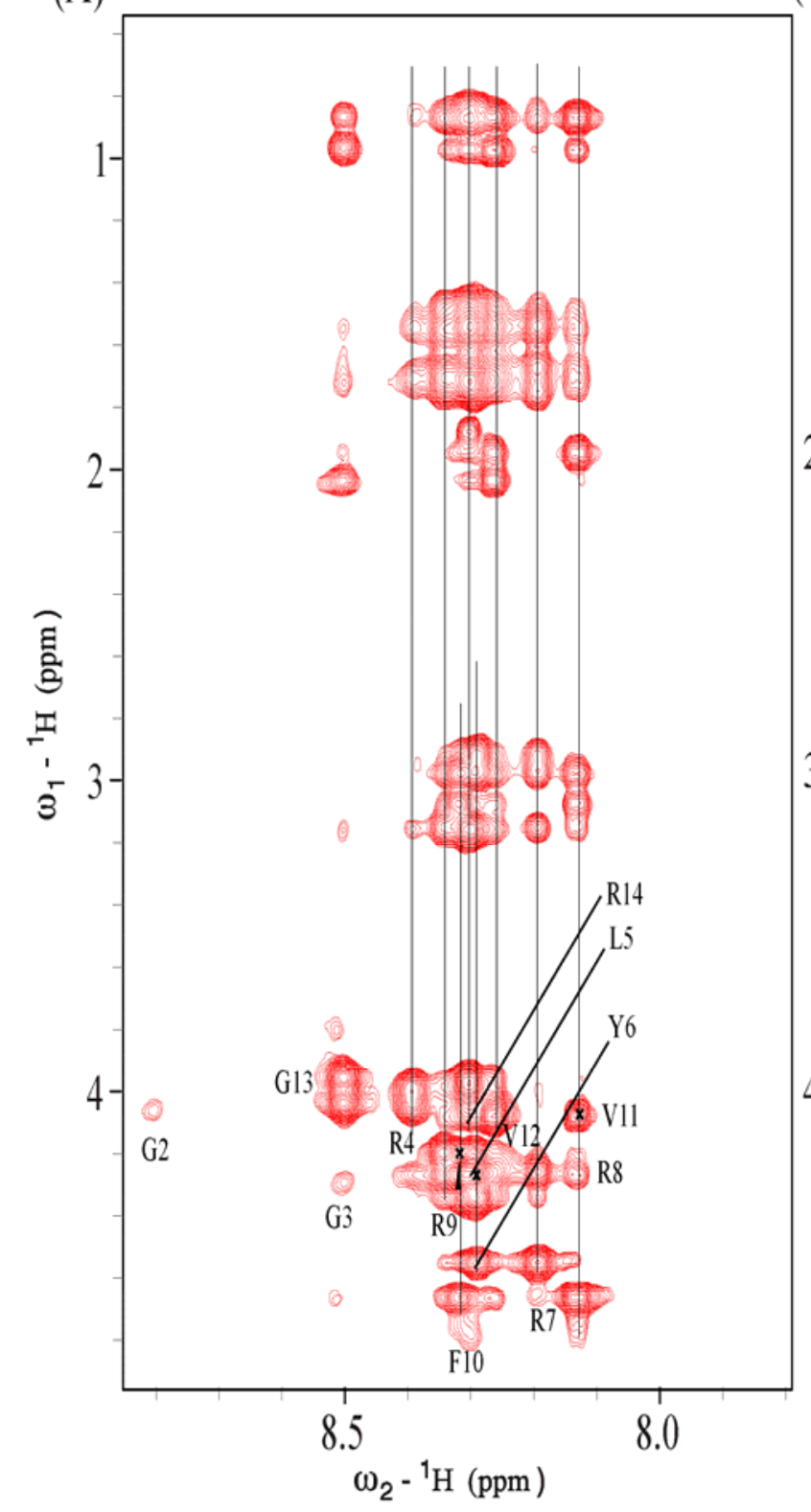

(B)

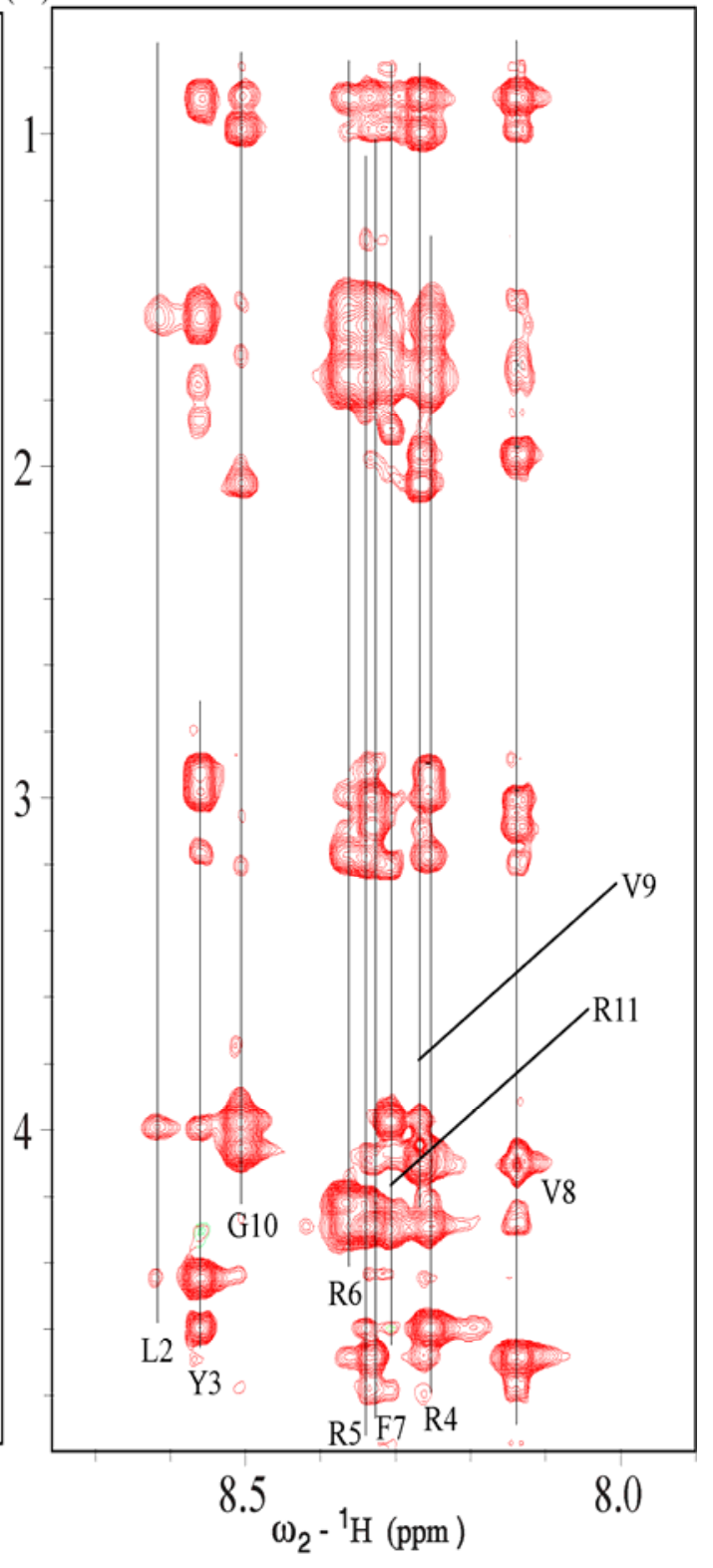

Figure 3.3-7: Sequence specific resonance assignment of finger print region of two dimensional ${ }^{1} \mathrm{H}-{ }^{1} \mathrm{H}$ tr-NOESY spectrum of (A) RR14 and (B) RR11 in LPS micelles at $298 \mathrm{~K}$. 
When compared to NOESY spectra in free state, RR14 and RR11 peptides, in the presence of LPS exhibited large number of side chain-side chain interactions mainly because of tr-NOE (Figure 3.3-8).
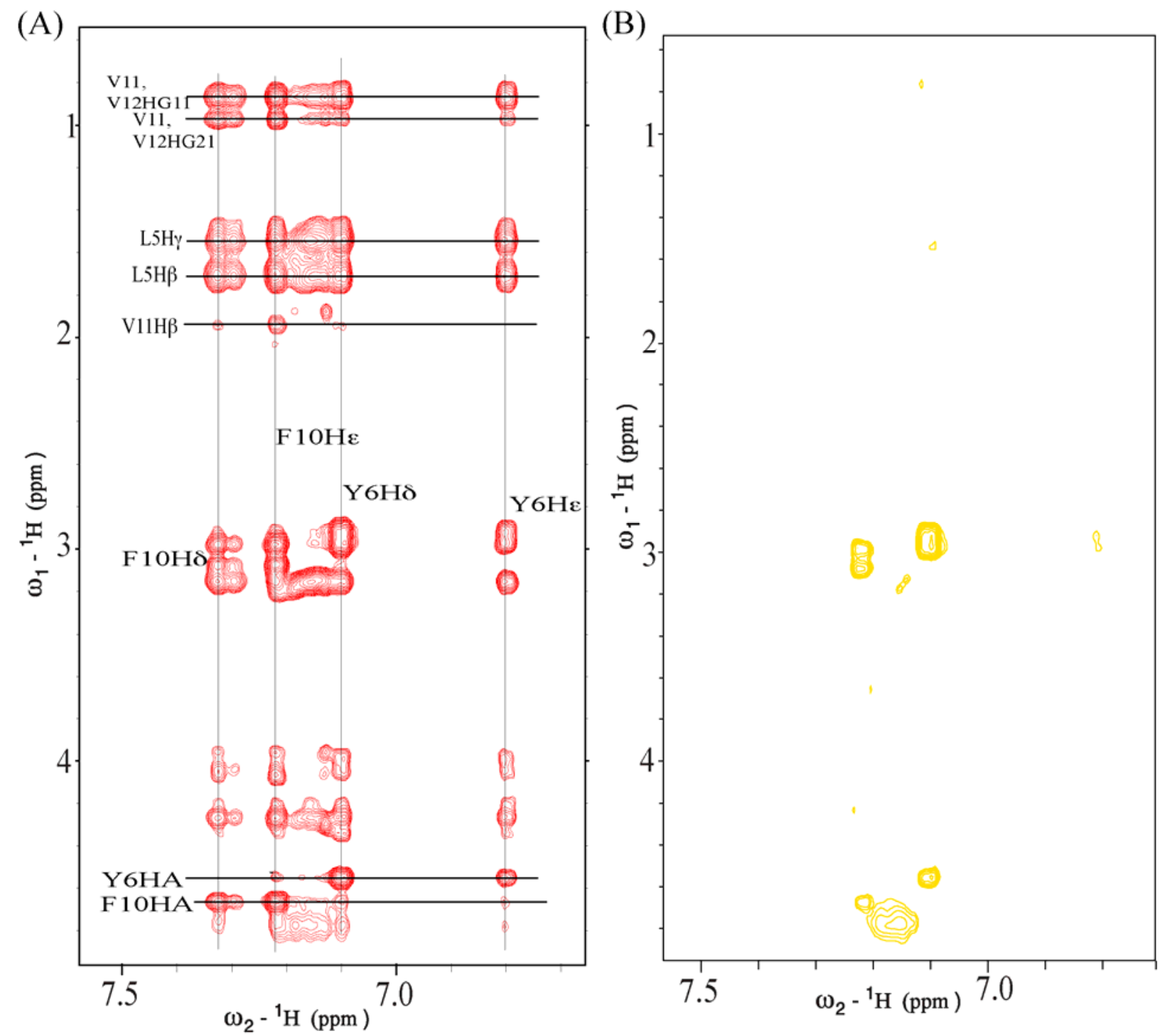

Figure 3.3-8: Selected region of two dimensional ${ }^{1} \mathrm{H}-{ }^{1} \mathrm{H}$ tr-NOESY spectrum highlighting long range aliphatic-aromatic side chain interactions (A) in comparison to the peptide RR14 in aqueous medium (B).

Careful examination of tr-NOESY spectra of RR14 in LPS micelles further disclosed several long range interaction between aromatic side chains of residues Y6, F10 with aliphatic side chains of L5, V11 and V12 (Figure 3.3-8, A). In the same manner, 
NOEs were also observed between aromatic $\mathrm{H} \delta$ and $\mathrm{H} \varepsilon$ side chains of Y6 and F10 (Figure 3.3-8, A) displaying $\pi-\pi$ interaction. All of these side chain interactions were found to be lacking for the peptide in free state (Figure 3.3-8, B). Figure 3.3-9 A summarizes the sequential and medium range NOE connectivities. As can be seen in Figure 3.3-9 B, the residues G3, R4, L5 in N-terminus and the residues V11, V12, G13, R14 in C-terminus exhibit long range NOEs. On the other hand, the residues in the middle R7, R8, R9 delineate only medium range NOEs. Such an arrangement suggests that the peptide RR14 (full length cysteine deleted analog of PG-1), adopts antiparallel beta hairpin even in the absence of disulphide bridges.

(A)

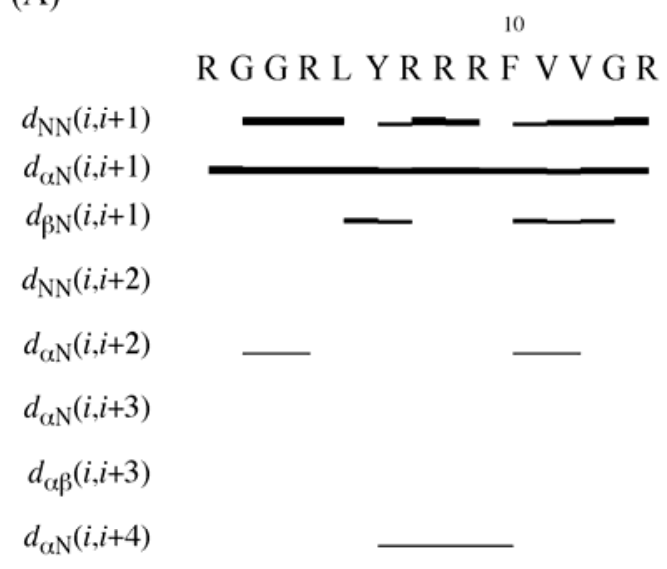

(B) 32

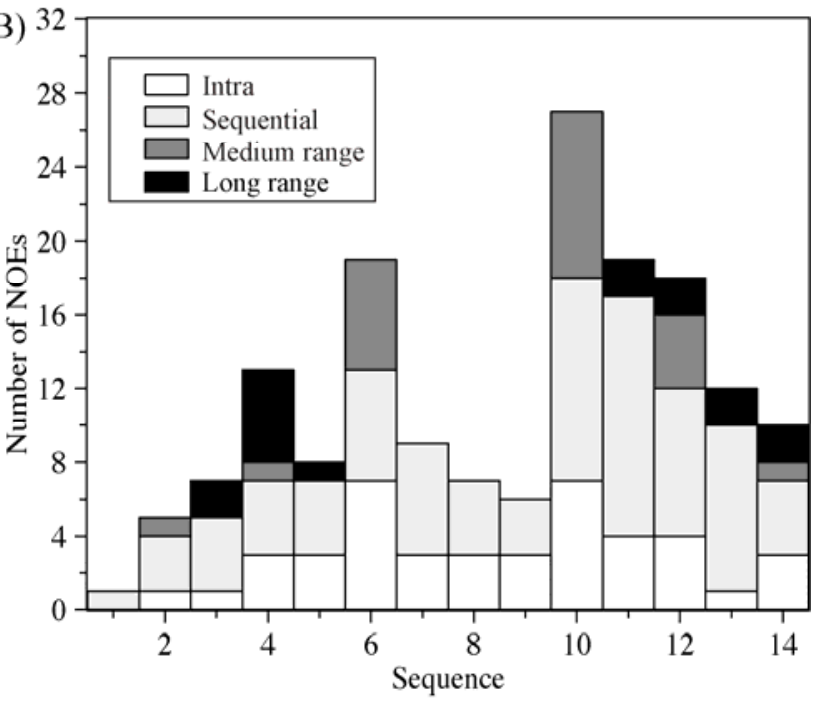

Figure 3.3-9: (A) NOE summary of RR14 in LPS micelles. (B) Dissemination of NOEs into sequential, medium and long range for RR14 in LPS micelles.

The tr-NOESY spectra of RR11 in LPS micelles also displayed several side chain-side chain NOEs (Figure 3.3-10, A) when compared to the peptide in free state (Figure 3.3-10, B). Most of the residues exhibited either sequential or medium range $(i-i+2)$ NOEs. Long range NOEs were delineated between aliphatic side chain delta protons of V8, V9 with aromatic side chain delta and eeta protons of Y3 and F6. But when compared to RR14, number of long range NOEs appeared limited to petite amounts. 
(A)

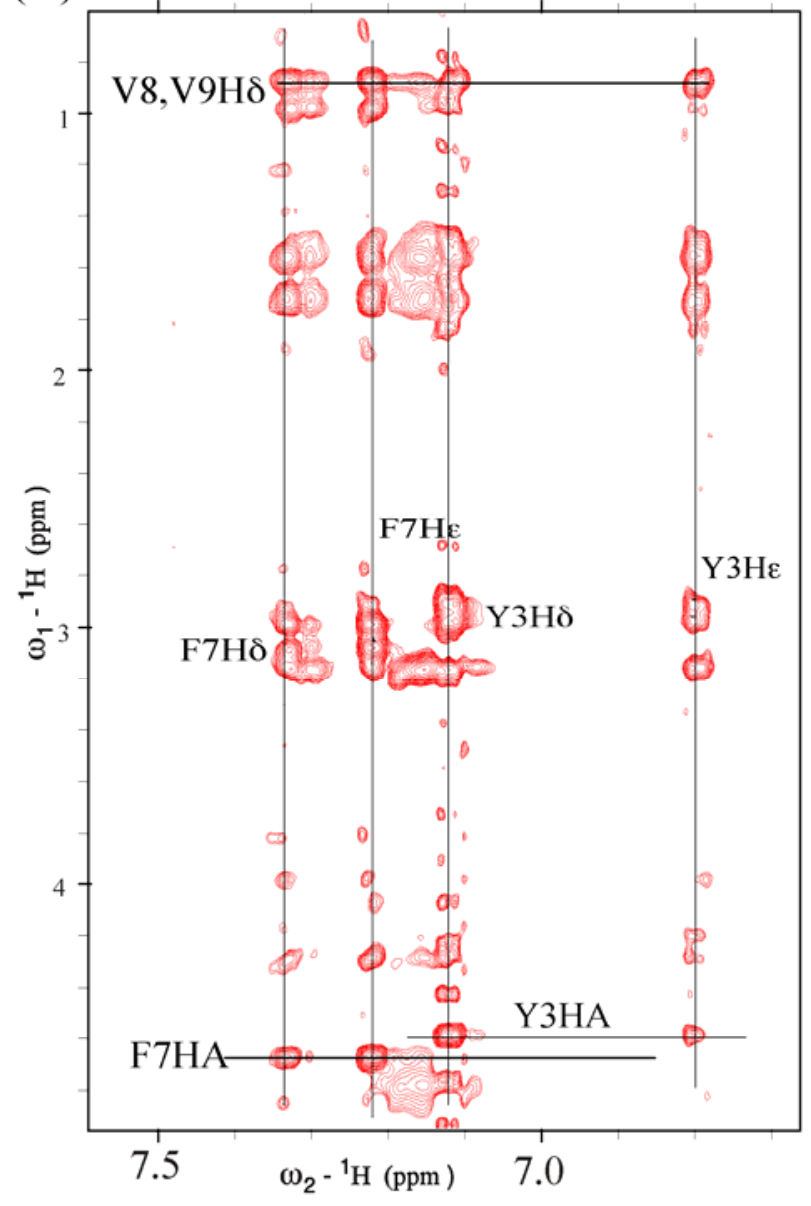

(B)

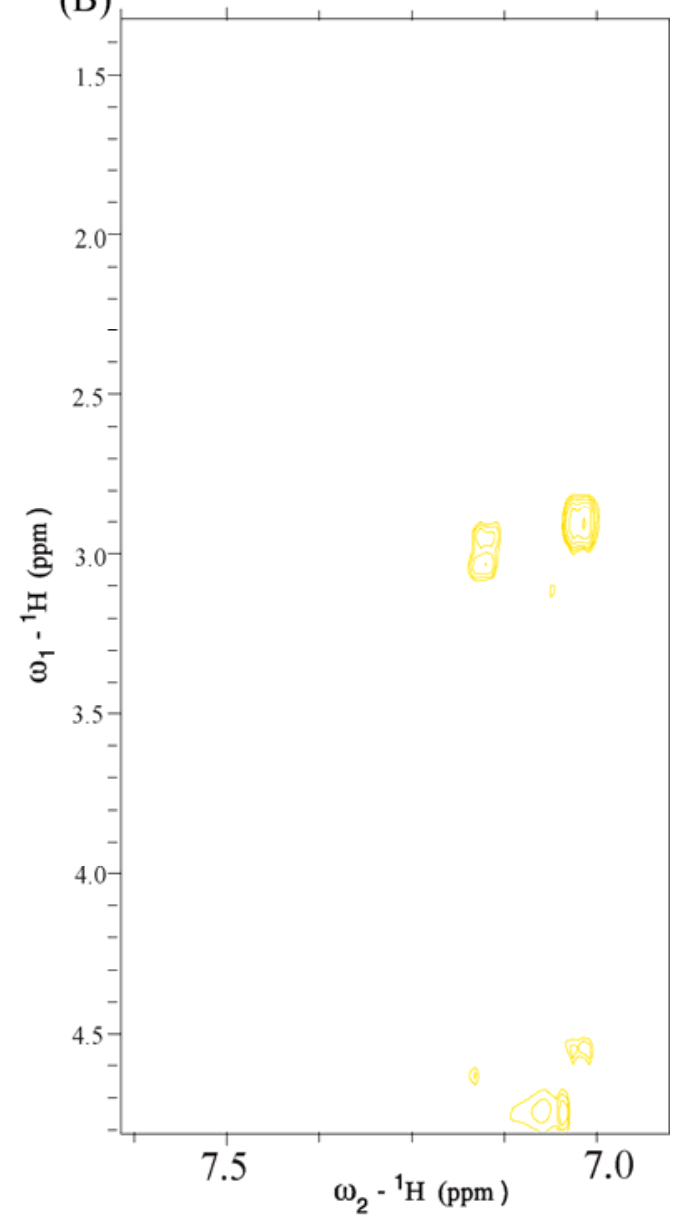

Figure 3.3-10: (A) Selected region of two dimensional ${ }^{1} \mathrm{H}-{ }^{1} \mathrm{H}$ tr-NOESY spectrum of RR11 in LPS micelles, highlighting long range aliphatic-aromatic side chain interactions, in comparison to the peptide in aqueous medium (B).

Further examination revealed that the residues R4, R5, R6, F7 and V8 delineated medium range NOEs of type $\mathrm{i}-\mathrm{i}+4$ and residues $\mathrm{Y} 3, \mathrm{~V} 9$ and $\mathrm{R} 11$ displayed long range NOEs (Figure 3.3-11,B). The medium range $\mathrm{i}-\mathrm{i}+4$ NOEs detected along with long range NOEs suggest the formation of antiparallel beta strands with turn in the middle. 
(A)

$$
\begin{aligned}
& d_{\mathrm{NN}}(i, i+1) \\
& d_{\alpha \mathrm{N}}(i, i+1) \\
& d_{\beta \mathrm{N}}(i, i+1) \\
& d_{\mathrm{NN}}(i, i+2) \\
& d_{\alpha \mathrm{N}}(i, i+2) \\
& d_{\alpha \mathrm{N}}(i, i+3) \\
& d_{\alpha \beta}(i, i+3) \\
& d_{\alpha \mathrm{N}}(i, i+4)
\end{aligned}
$$

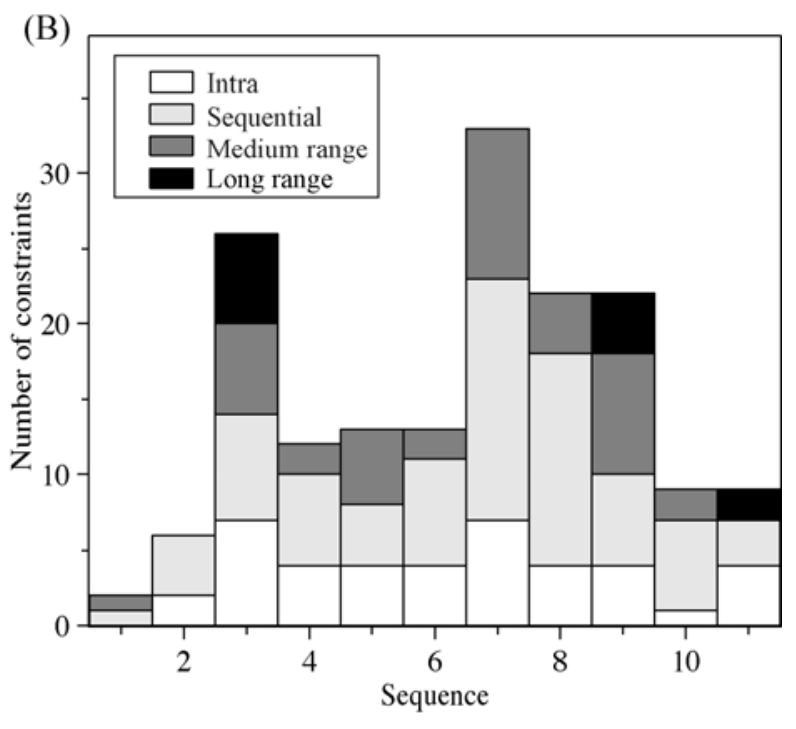

Figure 3.3-11: (A) NOE summary of RR11 in LPS micelles. (B) Dissemination of NOEs into sequential, medium and long range for RR11 in LPS micelles.
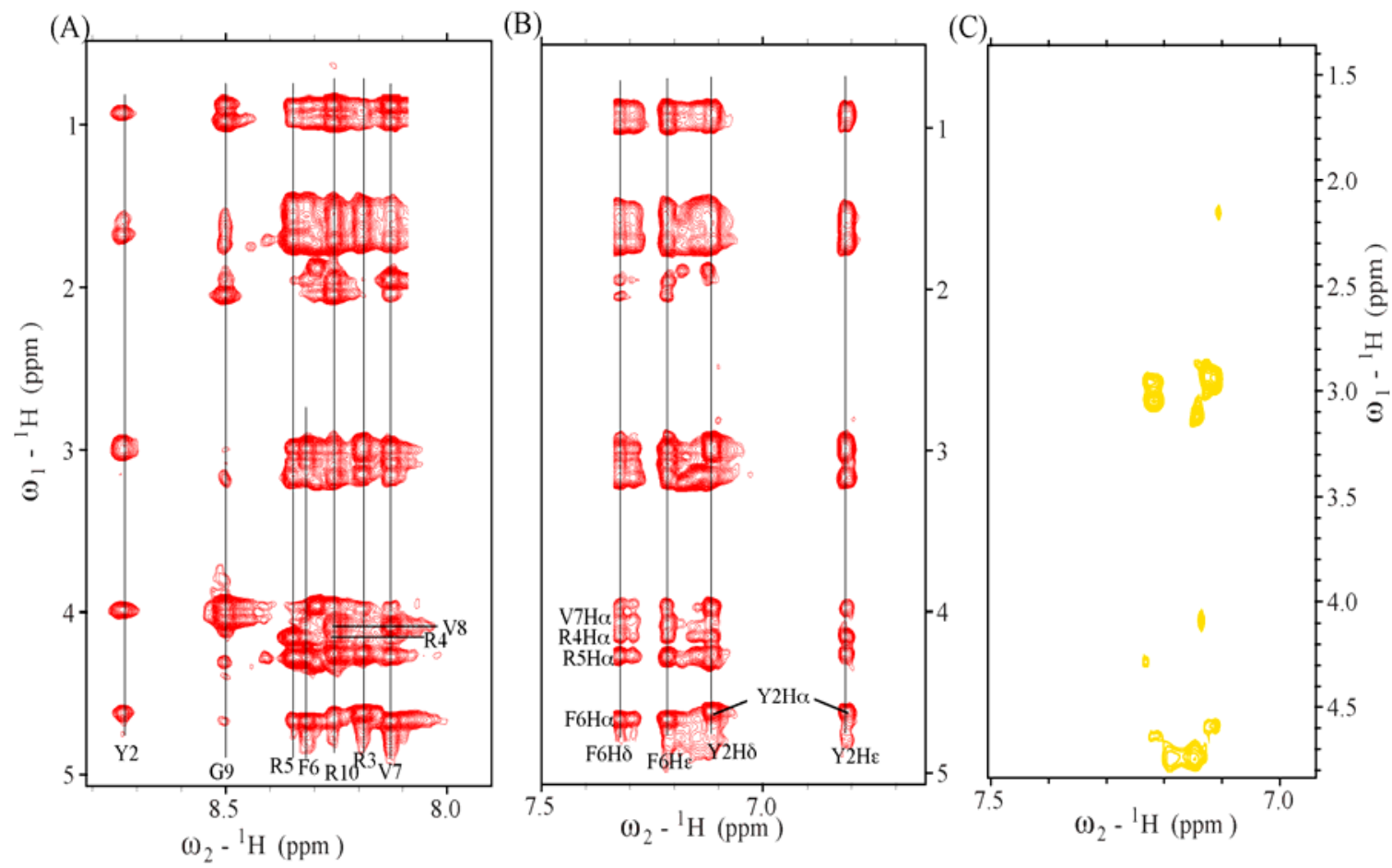

Figure 3.3-12: (A) Sequnece specific assignment of aminoacid residues of LR10 in two dimensional ${ }^{1} \mathrm{H}-{ }^{1} \mathrm{H}$ tr-NOESY spectrum. (B) Selected regions of two dimensional ${ }^{1} \mathrm{H}-{ }^{1} \mathrm{H}$ tr-NOESY spectrum displaying medium range aliphaticaromatic side chain interactions, in comparison to the peptide LR10 in aqueous medium (C). 
Inorder to examine the structural property of inactive LR10, tr-NOESY spectra were acquired for LR10 in the presence of LPS micelles. Comparison of tr-NOESY (Figure 3.3-12, B) and NOESY spectra (Figure 3.3-12, C) reveal that there were sidechainsidechain NOEs arising from the peptide in the presence of LPS. But the NOEs were of sequential and medium range of $i-i+2$ type. The spectrum was devoid of any long range NOEs.

Further the analysis of side chain-side chain NOEs disclosed that they were arising from aliphatic and aromatic interactions between L1 with Y2 and F6 with V7 and V8 (Figure 3.3-12, B). All these NOEs were of $i-i+2$ type (Figure 3.3-13) suggesting that the peptide LR10 adopts extended or beta conformation in the presence of LPS.

(A)

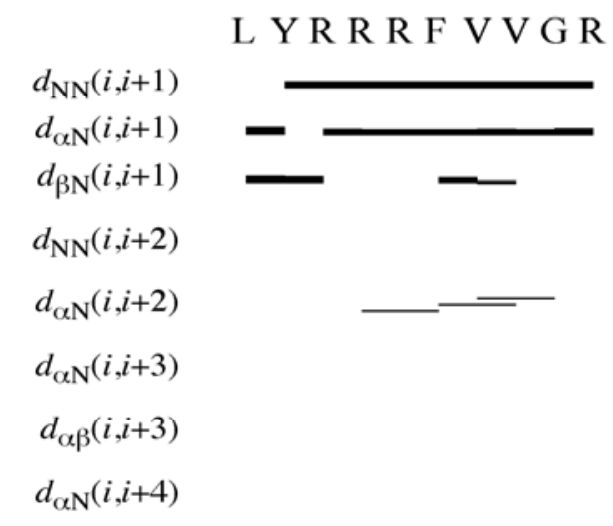

(B)

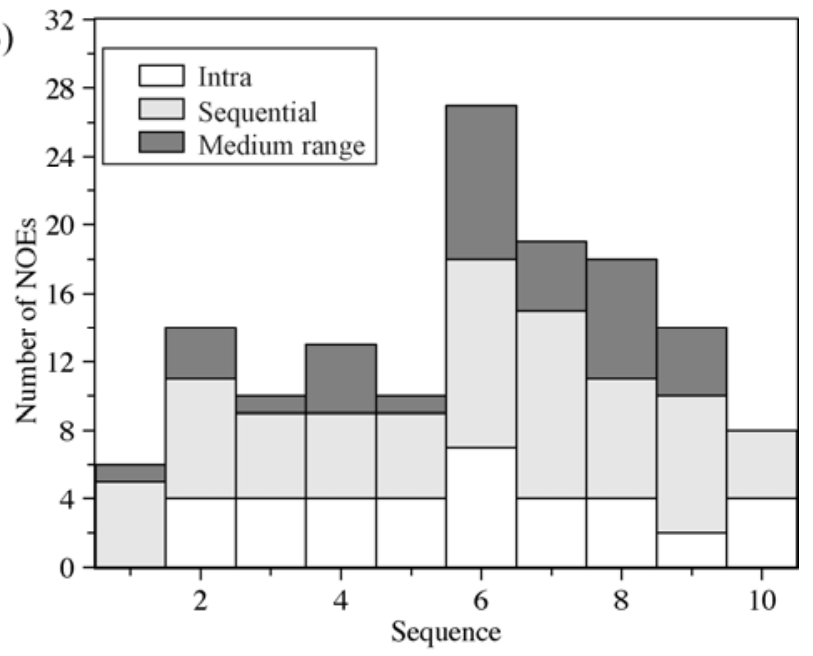

Figure 3.3-13: (A) NOE summary of LR10 in LPS micelles. (B) Dissemination of NOEs into sequential, medium range for LR10 in LPS micelles.

\subsubsection{Three dimensional structures of the peptides in LPS micelles:}

After complete assignment and analyses of tr-NOESY spectra, three dimensional structures of the peptides in the presence of LPS were calculated by simulated annealing. The intensity of the crosspeaks were converted to distance constraints and used for structure calculation. The summary of structural data used in determining three dimensional structures of the peptides in LPS micelles are presented in Table 3.3-4. 
Table 3.3-4: Summary of structural statistics of 20 lowest energy structures of RR14, RR11, and LR10.

\begin{tabular}{|c|c|c|c|}
\hline & RR14 & RR11 & LR10 \\
\hline \multicolumn{4}{|l|}{ Distance restraints } \\
\hline intraresidue $(|i-j|=0)$ & 43 & 41 & 37 \\
\hline sequential $\quad(|i-j|=1)$ & 40 & 37 & \\
\hline medium range $(2 \leq|i-j| \leq 4)$ & 11 & 20 & 34 \\
\hline long range $(|i-j| \leq 5)$ & 8 & 6 & 17 \\
\hline total NOE constraints & 102 & 104 & 0 \\
\hline & & & 88 \\
\hline \multicolumn{4}{|l|}{ Deviation from mean structure } \\
\hline backbone atoms $\left(\mathrm{N}, \mathrm{C} \alpha, \mathrm{C}^{\prime}\right)(\AA)$ & 0.4 & 0.03 & 0.27 \\
\hline heavy atoms $(\AA)$ & 1.53 & 0.98 & 1.19 \\
\hline \multicolumn{4}{|l|}{$\begin{array}{l}\text { Ramachandran plot for the mean } \\
\text { structure }\end{array}$} \\
\hline $\begin{array}{l}\% \text { residues in the most favourable and } \\
\text { additionally allowed regions }\end{array}$ & 100 & 100 & 71.5 \\
\hline $\begin{array}{l}\% \text { residues in the generously allowed } \\
\text { region }\end{array}$ & 0 & 0 & 28.5 \\
\hline$\%$ residues in the disallowed region. & 0 & 0 & 0 \\
\hline
\end{tabular}

An ensemble of twenty lowest energy structures was generated using the parameters presented in statistics table (Figure 3.3-14). The rmsd for backbone of 20 lowest energy structures were $0.4,0.03$ and 0.27 for RR14, RR11 and LR10 respectively (Table 3.3-4). The calculated structures were validated using Ramachandran plot. All of the residues were found to occupy the most favourable and additionally allowed regions except in LR10, where in two residues were found to occupy the generously allowed region (Table 3.3-4). 
(A)

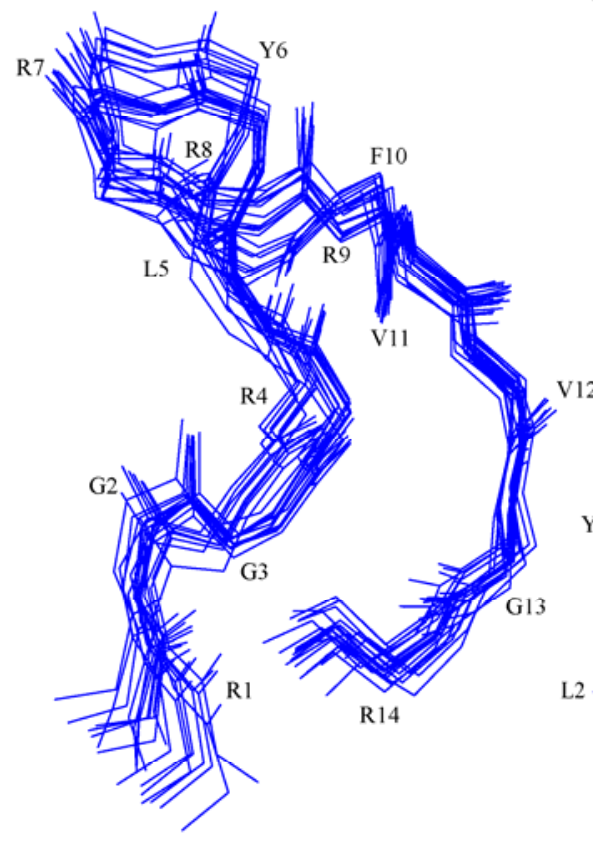

(B)

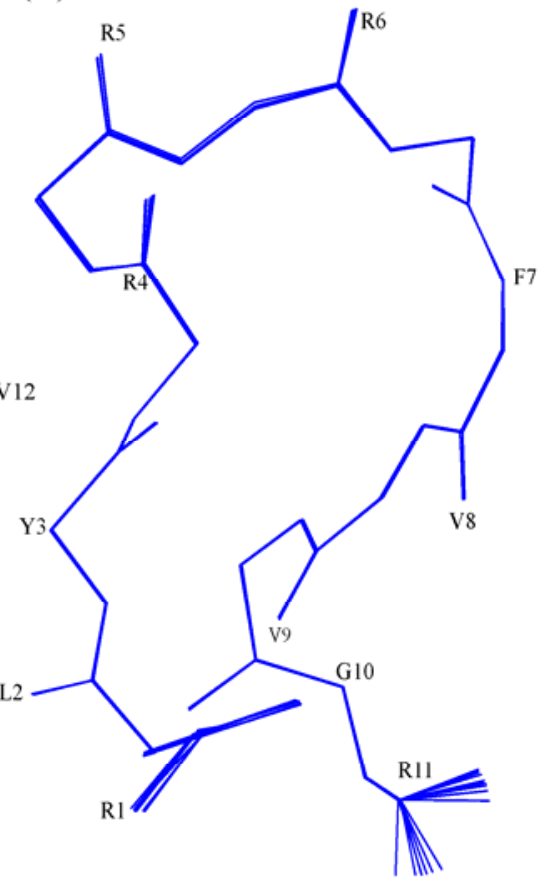

(C)

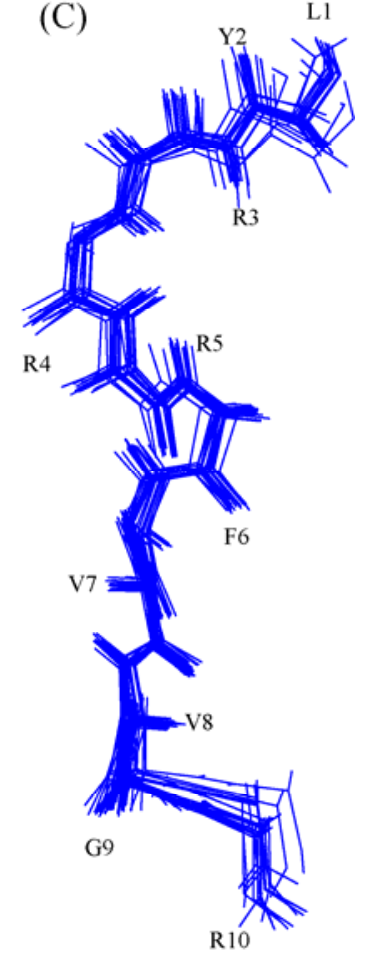

Figure 3.3-14: Ensemble of twenty lowest energy structures of (A) RR14, (B) RR11 and (C) LR10. Figures generated by MOLMOL [179].

After structure validation, the peptide structures were analysed for specific interactions. Single model representation of RR14 clearly visualized $\beta$-hairpin structure delineating the aromatic stacking interaction between Y6 and F10 with R7, R8 and R9 forming turn (Figure 3.3-15, A). This aromatic stacking interaction imparts amphipathic conformation to the peptide with the basic Arg residues on one side and hydrophobic Y6, F10, V11 and V12 on the opposite side. RGG deleted RR11 also displayed long range NOEs between Y3, V9 and R11 forming antiparallel $\beta$-hairpin (Figure 3.3-15, B) like full length RR14. Since the flexible region RGG is deleted, the aromatic stacking interaction between Tyr and Phe is lost leading to the formation of inactive peptide. In parallel, when the flexible RGGR domain is completely removed, LR10 peptide loses the potency to form $\beta$-hairpin leading to the formation of extended or beta conformation (Figure 3.3-15, C). The specific interaction needed to induce attraction towards negatively charged bacterial membrane could not be formed leading to reduced binding of the peptide to LPS micelles. 

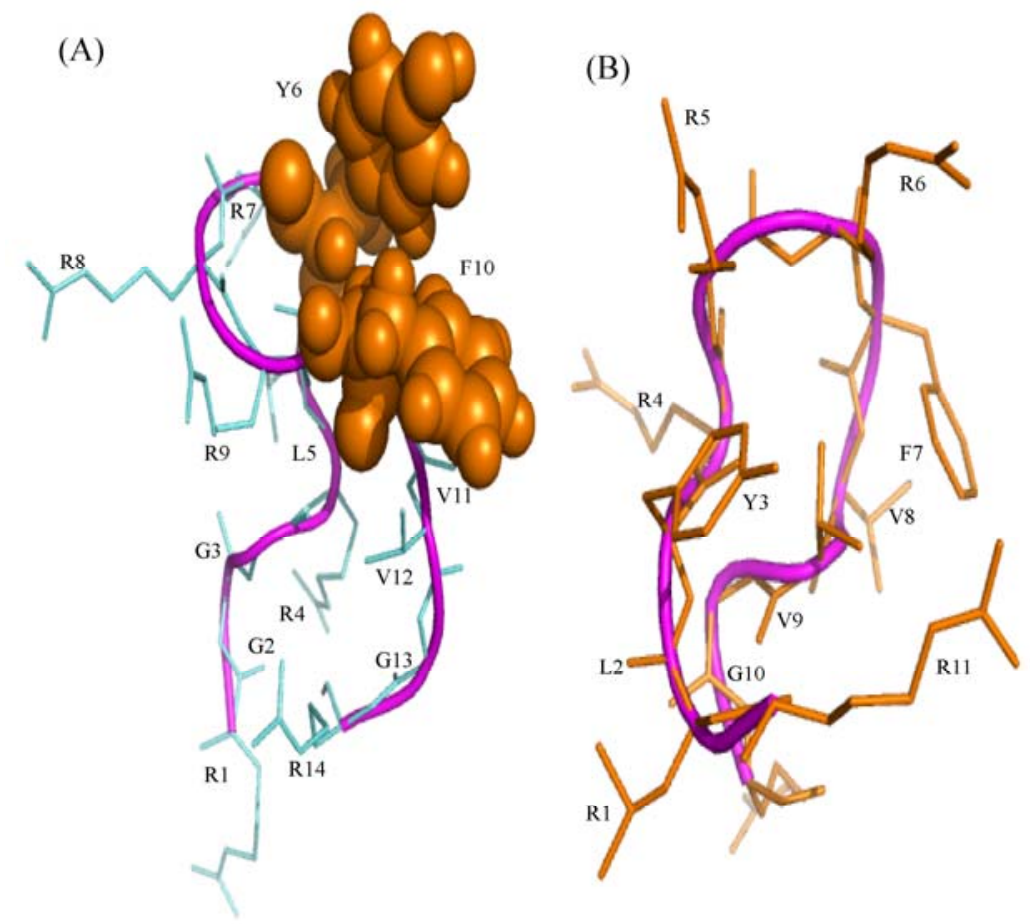

(C)

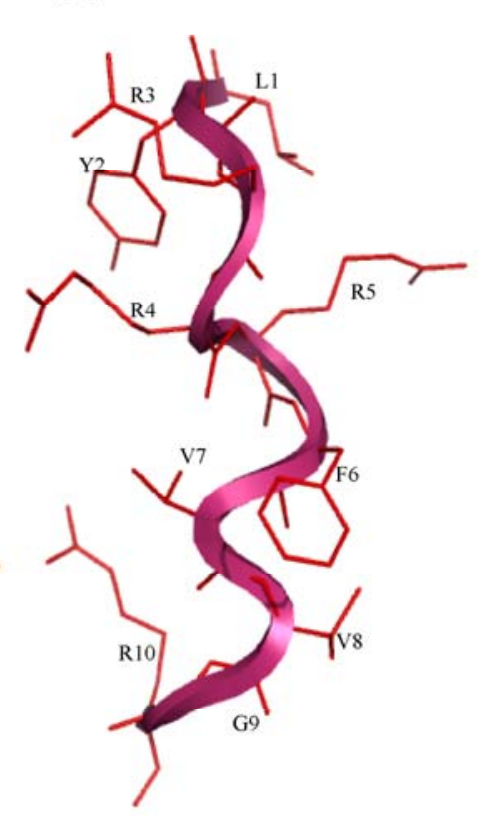

Figure 3.3-15: Single model representations of calculated structure of (A) RR14, (B) RR11 and (C) LR10.

Electrostatic surface representation of RR14 clearly explains the position of various Arg residues in the antiparallel $\beta$-hairpin. In RR14, the first R1 and last R14 residue together initiates the electrostatic attraction of the peptide towards anionic LPS molecules followed by combined electrostatic and hydrophobic forces exerted by aromatic stacking with the arginine turn disrupting the bacterial cell completely (Figure 3.3-16, A). In the case of RR11 also the first R1 and last R11 initiates the electrostatic attraction, but the hydrophobic forces needed to disrupt the cells cannot be exerted. (Figure 3.3-16, B) Lack of potential cationic and hydrophobic surface in LR10 makes the peptide completely inactive (Figure 3.3-16, C). 


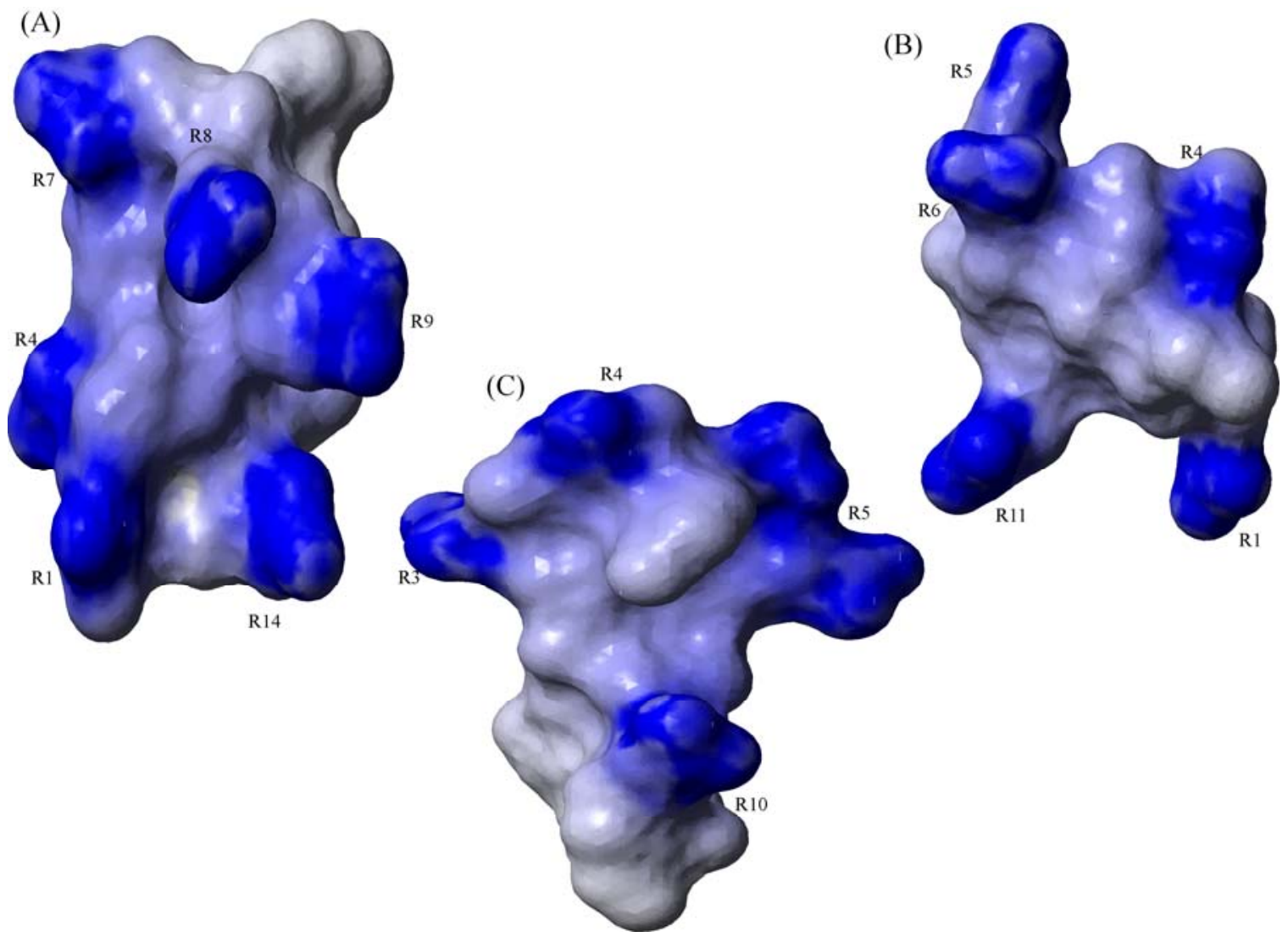

Figure 3.3-16: Electrostatic surface representation of (A) RR14, (B) RR11 and (C) LR10. Positions of Arginine residues are marked for reference. Figures generated by MOLMOL [179].

\subsubsection{Structure determination of peptides in DPC micelles:}

In order to check the choice of peptides towards anionic lipids, two dimensional ${ }^{1} \mathrm{H}-{ }^{1} \mathrm{H}$ NOESY and ${ }^{1} \mathrm{H}-{ }^{1} \mathrm{H}$ TOCSY spectra were obtained for the peptides in $\mathrm{d}_{38}$-DPC micelles. The peptide: DPC ratio was maintained at 1:250 to ensure the complete binding of peptide with micelles. Unlike tr-NOESY spectra of the peptides in LPS, the NOESY spectra in DPC micelles displayed only intraresidue and sequential $(\mathrm{i}-\mathrm{i}+1)$ NOEs (Figure 3.3-17). Careful examinations of the spectra revealed no medium or long range NOEs present for all of the three peptides. This confirms that the peptide does not acquire any conformation in zwitterionic micelle and is largely specific for negatively charged lipids. 
(A)

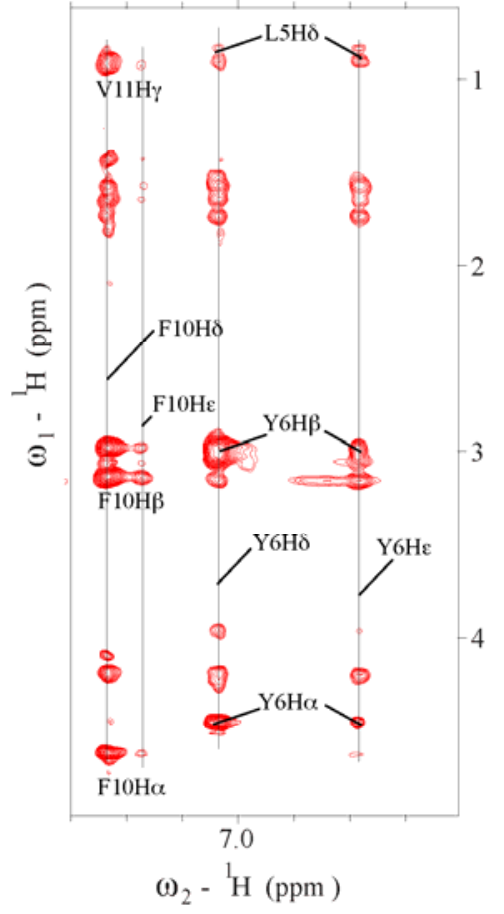

(B)

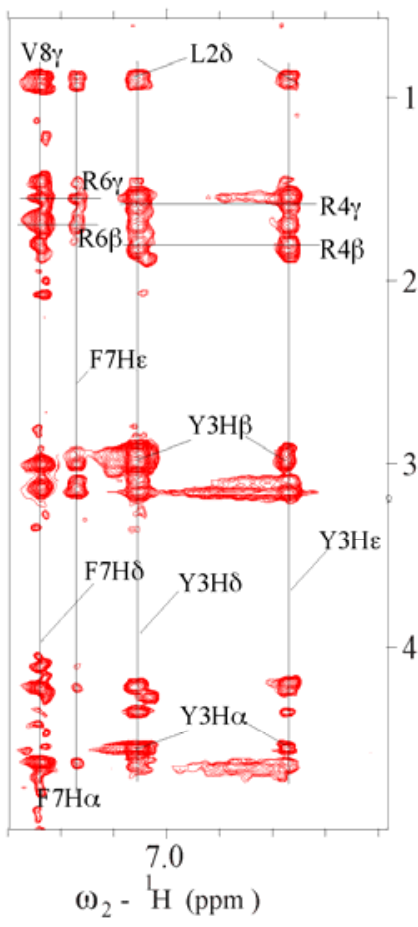

(C)

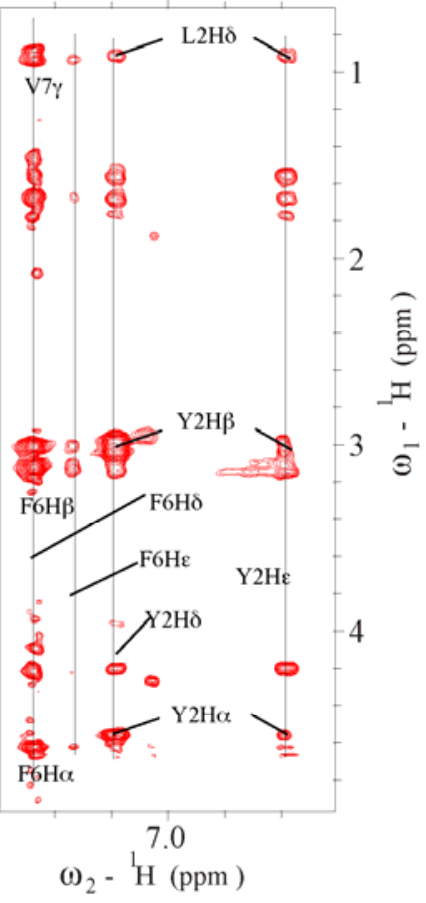

Figure 3.3-17: Selected region of two dimensional ${ }^{1} \mathrm{H}-{ }^{1} \mathrm{H}$ NOESY spectra of (A) RR14, (B) RR11 and (C) LR10 in $\mathrm{d}_{38}$ DPC micelles at $308 \mathrm{~K}$.

\subsubsection{Discussion:}

Protegrins are a group of naturally occurring antimicrobial peptides isolated from porcine leucocytes [174] that has amidated C-terminal. Protegrin-1 (PG-1) is composed of 18 aminoacid residues with 6 cationic arginine and 4 cysteine residues. This peptide adopts antiparallel $\beta$-sheet with the help of disulphide bridges between C6, C8 and C15, C13 respectively [175]. Because of its short size with broad antimicrobial activity even in physiological salt conditions [34], PG-1 has attracted lot of attention towards development of new antibacterial agents. Alanine mutations of all cystiene reveal that the peptide loses its ability to form beta hairpin structure with concomitant loss of antibacterial activity [34, 180]. On the other hand if either of these cysteine residues are retained, then the peptide retains its activity even in physiological salt conditions [34]. All of these studies reveal the importance for the peptide to adopt antiparallel $\beta$-hairpin structure and that replacement of some arginine residues does not really affect membrane perturbing activity [180]. 
Structural studies at atomic resolution level in various biomembranes have shed light on the importance of disulphide bonds and the aromatic packing interactions. Hence structure-activity correlation studies of cysteine deleted analogs of PG-1 peptide were carried out.

Three dimensional structure determination of RR14, full length cystiene deleted analog revealed that the peptide adopts antiparallel $\beta$-hairpin conformation only in the presence of anionic membrane. Tr-NOESY spectrum of the peptide in LPS micelles delineated several long range NOEs between G3-R14, R4-V12, L5-V11 and Y6-F10. The aromatic side chain interactions that involve $\pi-\pi$ or cation- $\pi$ plays an important role in insertion of AMPs in membranes [181]. The side chain interactions of Y6 and F10 that displays $\pi-\pi$ interaction has been found to be instrumental in bringing the two strand of RR14 closer. This close proximity of $\pi-\pi$ packing in one side with the cationic residues grouped on the opposite side imparts amphipathic conformation to RR14. The structure determination in LPS micelles has led to better understanding of the peptide in outer membrane permeabilization and LPS neutralization.

Activity studies of RR14 reveal that the peptide displays broad spectrum of activity against both Gram negative and Gram positive bacteria. Even in physiological conditions where the testing medium was supplemented with $150 \mathrm{mM} \mathrm{NaCl}$, RR14 peptide shows moderate killing activity. It exhibits considerable ability to permeabilize both the membranes of bacteria and LPS micelle dissociating activity. RR14 also binds to LPS very tightly with $\mathrm{K}_{\mathrm{d}}$ value of $0.35 \mu \mathrm{M}$. The binding reaction was found to be exothermic that primarily involves ionic interaction. The electrostatic surface distribution of RR14 in LPS micelles demonstrates that all six arginine residues are packed together.

When the first three residues were deleted, RR11 peptide also adopted antiparallel $\beta$ hairpin in the presence of LPS micelles. But the aromatic stacking interaction was found to be lacking. Unlike RR14, RR11 displayed only fewer long range NOEs between residues in $\mathrm{N}$ and $\mathrm{C}$-terminal. The long range aromatic-aliphatic interaction between Y3 in N-terminal and V9 and R11 in C-terminal has confined antiparallel $\beta$ hairpin structure to the peptide. Because of this combination, F7 has occupied the turn position. When RGGR motif in N-terminal was deleted, LR10 peptide adopted 
extended or beta strand like conformation in LPS micelles. No long range NOEs or aliphatic-aromatic side chain interactions were detected.

Activity studies of RR11 and LR10 revealed the importance of antiparallel $\beta$ - hairpin conformation with aromatic stacking interaction. In the absence of disulphide bridges, aromatic stacking interaction between Tyr and Phe has contributed to the activity of RR14 that was found to be lacking in RR11 and LR10 peptides. The ample flexibility of the first Arg residue was found to be the driving force to initiate electrostatic binding with LPS. When first 3 residues RGG was deleted, RR11 show only moderate killing activity which decreases further in the presence of $\mathrm{NaCl}$. The flexibility to initiate ionic interactions was contributed by $\mathrm{R} 4$ that is first residue in RR11. The dissociation constant $\mathrm{K}_{\mathrm{d}}$ value was found to be $0.9 \mu \mathrm{M}$, which is 3 fold more than RR14. The binding reaction was also found to be exothermic with ionic interactions dominating. When this Arg residue was further deleted, the ability to fold into $\beta$ hairpin was completely lost and the peptide adopted extended conformation. This has abolished the potential to form aliphatic-aromatic side chain interactions also that led to the complete destruction in killing activity. Irrespective of the conformation, the binding was found to be exothermic reaction. Yet the $K_{d}$ value was as high as $3 \mu \mathrm{M}$ that explains the inactiveness of the peptide.

On the whole, in the absence of disulphide bridges, the RR14 peptide, with its amphipathic conformation of beta hairpin is found to be active against bacteria. Eventhough RR11 peptide acquires beta hairpin conformation; the propensity of forming amphipathic distribution is decreased with deletion of N-terminal RGG residues. Hence the activity also decreases. With the removal of RGGR residues in LR10, it cannot form beta hairpin and exhibit extended or random coil structure with no activity against bacteria. Hence the studies on Cys-deleted analogs of PG-1 shed light on the unique structural features in recognition of LPS and its neutralization even after removal of disulphide bridges. 


\subsection{DESIGN OF ARGININE RICH SALT RESISTANT PEPTIDES}

\subsubsection{Peptide design:}

Antimicrobial peptides can be categorized based on different properties. One of the most widely used classifications is based on the conformation it adopts on membrane interactions. There are 3 different classes namely (i) alpha helix as in Magainins and Melittin, (ii) beta hairpin or beta sheet stabilized by disulfide bridges as in Defensins and Protegrins and (iii) extended structures rich in aminoacids like proline or tryptophan or histidine as in Tritripticin and Indolicidin [14]. Nonetheless the structural diversity, the antimicrobial peptides are generally characterized by the presence of cationic charged residues balanced with hydrophobicity. Hence arginine and tryptophan residues occupy prominently higher percentage in antimicrobial peptides [168]. Apart from the ability of Trp and Arg pair to engage in cation $-\pi$ interactions, Trp residues are preferred in interfacial region of lipid bilayers and Arg residues are preferred for its hydrogen bond forming capability of its guanidium side chains [168]. In order to be therapeutically applicable, AMPs need to be active in physiological solutions containing salt and serum.

Table 3.4-1: Primary structure of designed peptides used in this study.

\begin{tabular}{|l|l|}
\hline Peptides & Primary structure \\
\hline RRW12 & R-R-L-I-W-L-I-L-R-L-L-R \\
\hline KKW12 & K-K-L-I-W-L-I-L-K-L-L-K \\
\hline RRL12 & R-R-L-I-R-L-W-L-R-L-L-R \\
\hline RR12 & R-R-L-I-R-L-I-L-R-L-L-R \\
\hline
\end{tabular}

Many antimicrobial peptides suffer from susceptibility to high salt concentrations or physiological salt concentrations. In conditions like cystic fibrosis, where the bronchopulmonary fluids show increased presence of $\mathrm{Cl}^{-}$ion, the efficiency of AMPs decreases. Hence organisms like P.aeruginosa colonizes inside lungs [182]. Most of the salt resistance AMPs that were derived from natural sources was found to adopt $\beta$ hairpin conformations stabilized with disulfide bridges as in Protegrins, Bactenecins 
$[183,184]$. When these oligomeric peptides were linearized with disulfide bond removal, then the salt resistance activity was found to be significantly diminished [34].
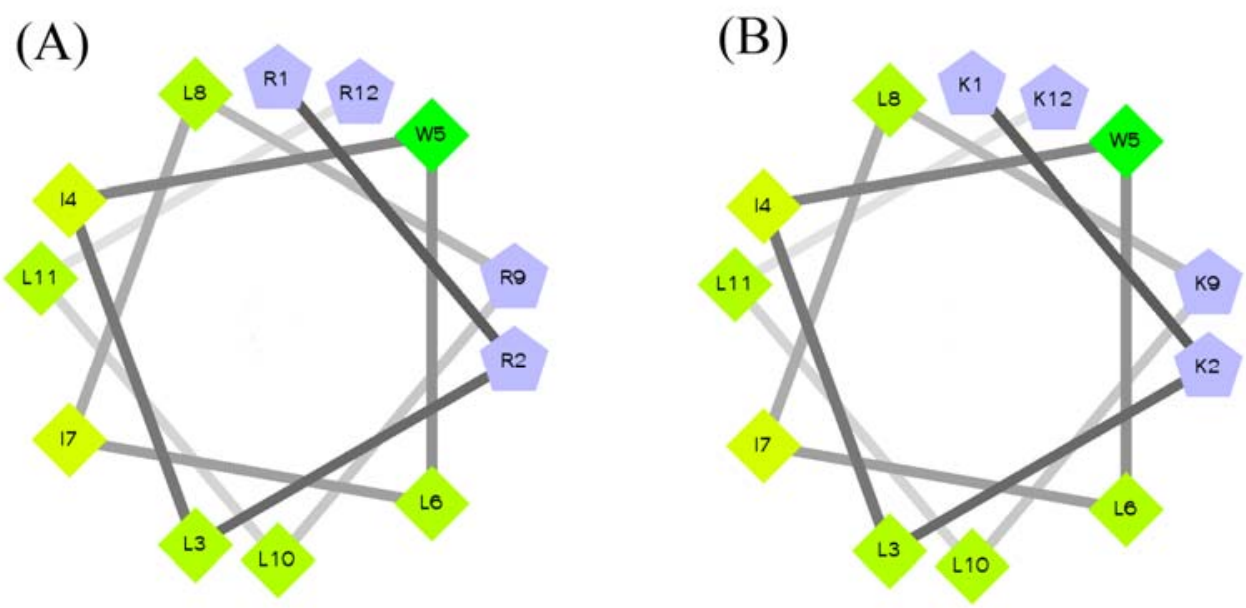

(C)

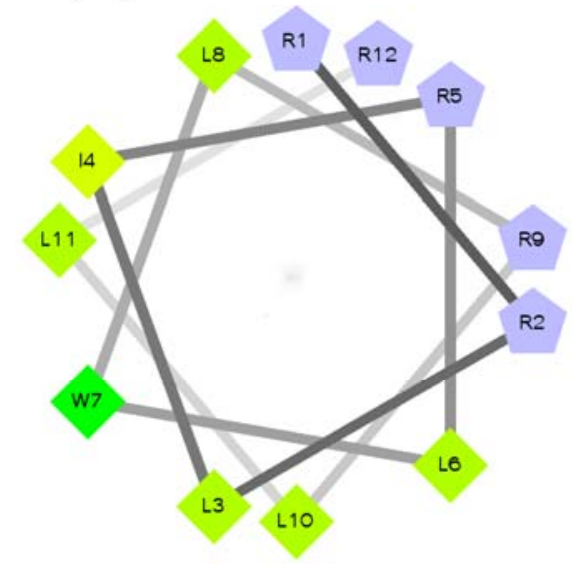

(D)

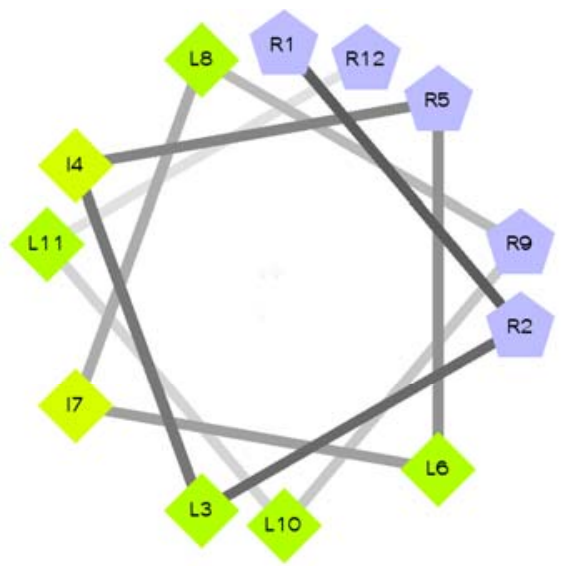

Figure 3.4-1: Helical wheel projection of designed peptides (A) RRW12, (B) KKW12, (C) RRL12 and (D) RR12.

Much effort has been taken in recent times to engineer AMPs that are resistant to physiological salt concentrations. In human $\beta$-defensin 3 , RRKK motif has been identified to be responsible for salt tolerance [185]. Similar motif of KKRR has also 
been identified in Protegrin derived peptidomimetics targeted towards outer membrane biogenesis in P.aeruginosa [186]. When HBD-3 RRKK motif was engineered with tryptophan and arginine residues in 10-residue decamers, two peptides D5 and D6 was found to be membrane active and salt tolerant [187]. But this arginine-tryptophan rich peptide adopted extended structure in membrane environments. Taken together all these results, peptides were designed that are rich in arginine and tryptophan residues with incorporation of leucine and isoleucine residues that can promote alpha helical conformations (Table 3.4-1). These peptides are designed based on physicochemical properties of aminoacids enabling membrane interactions.

In RRW12 peptide, the Trp residue was placed in cationic face whereas in RRL12, the Trp residue was placed in hydrophobic face (Figure 3.4-1). In KKW12, the Arg residues were replaced with Lys and in RR12 no Trp residue was present (Figure 3.4-1).

\subsubsection{Minimum inhibitory concentration (MIC) determination:}

The ability of the designed peptides to exhibit broad spectrum of activity was analysed by testing the peptides against four representative Gram negative and Gram positive strains. The experiment was also performed either in the absence of $\mathrm{NaCl}$ or with 150 $\mathrm{mM} \mathrm{NaCl}$ and $300 \mathrm{mM} \mathrm{NaCl}$ to determine salt sensitivity of the designed peptides.

All of the designed peptides irrespective of the presence and positioning of $\operatorname{Trp}$ residue were found to be active against Gram negative bacteria (

Table 3.4-2). Inclusion of physiological salt concentration of $150 \mathrm{mM} \mathrm{NaCl}$ or 300 $\mathrm{mM} \mathrm{NaCl}$ does not hinder the activity. In the case of RRW12 and RRL12, the peptide shows improved activity against P.aeruginosa in the presence of $300 \mathrm{mM} \mathrm{NaCl}$ concentration, whereas the activity of KKW12 and RR12 remain unaffected (

Table 3.4-2). 
Table 3.4-2: Determination of MIC $(\mu \mathrm{M})$ values for designed peptides in Gram negative bacteria examined in MH broth (MHB), MH broth supplemented with $150 \mathrm{mM} \mathrm{NaCl}$ (MHB 150) and $300 \mathrm{mM} \mathrm{NaCl}$ (MHB 300).

\begin{tabular}{|c|c|c|c|c|c|c|c|c|c|c|c|c|}
\hline & \multicolumn{3}{|c|}{ E.coli } & \multicolumn{3}{|c|}{$\begin{array}{l}\text { P.aeruginosa } \\
\text { ATCC27853 }\end{array}$} & \multicolumn{3}{|c|}{$\begin{array}{l}\text { K.pnuemoniae } \\
\text { ATCC13883 }\end{array}$} & \multicolumn{3}{|c|}{$\begin{array}{l}\text { S.enterica } \\
\text { ATCC14028 }\end{array}$} \\
\hline & $\begin{array}{l}\mathrm{MH} \\
\mathrm{B}\end{array}$ & $\begin{array}{l}\text { MH } \\
\text { B } \\
150\end{array}$ & $\begin{array}{l}\text { MH } \\
\text { B } \\
300\end{array}$ & $\begin{array}{l}\text { MH } \\
\text { B }\end{array}$ & $\begin{array}{l}\text { MH } \\
\text { B } \\
150\end{array}$ & $\begin{array}{l}\text { MH } \\
\text { B } \\
300\end{array}$ & $\begin{array}{l}\text { MH } \\
\text { B }\end{array}$ & $\begin{array}{l}\text { MH } \\
\text { B } \\
150\end{array}$ & $\begin{array}{l}\text { MH } \\
\text { B } \\
300\end{array}$ & $\begin{array}{l}\text { MH } \\
\text { B }\end{array}$ & $\begin{array}{l}\text { MH } \\
\text { B } \\
150\end{array}$ & $\begin{array}{l}\text { MH } \\
\text { B } \\
300\end{array}$ \\
\hline RRW12 & 8 & 8 & 8 & 8 & 4 & 4 & 4 & 4 & 8 & 8 & 4 & 8 \\
\hline $\begin{array}{l}\text { KKW1 } \\
2\end{array}$ & 4 & 4 & 8 & 4 & 2 & 4 & 4 & 8 & 8 & 4 & 8 & 8 \\
\hline RRL12 & 8 & 8 & 8 & 4 & 4 & 2 & 4 & 4 & 4 & 8 & 4 & 2 \\
\hline$\overline{R R 12}$ & 4 & 4 & 4 & 4 & 4 & 4 & 4 & 4 & 2 & 4 & 8 & 2 \\
\hline
\end{tabular}

Table 3.4-3: Determination of MIC $(\mu \mathrm{M})$ values for designed peptides in Gram positive bacteria examined in MH broth (MHB), MH broth supplemented with $150 \mathrm{mM} \mathrm{NaCl}$ (MHB 150) and $300 \mathrm{mM} \mathrm{NaCl}$ (MHB 300).

\begin{tabular}{|c|c|c|c|c|c|c|c|c|c|c|c|c|}
\hline & \multicolumn{3}{|c|}{ B.subtilis } & \multicolumn{3}{|c|}{$\begin{array}{l}\text { S.aureus } \\
\text { ATCC25923 }\end{array}$} & \multicolumn{3}{|c|}{$\begin{array}{l}\text { S.pyogenes } \\
\text { ATCC19615 }\end{array}$} & \multicolumn{3}{|c|}{$\begin{array}{l}\text { E.faecalis } \\
\text { ATCC29212 }\end{array}$} \\
\hline & $\begin{array}{l}\mathrm{MH} \\
\mathrm{B}\end{array}$ & $\begin{array}{l}\text { MH } \\
\text { B } \\
150\end{array}$ & $\begin{array}{l}\text { MH } \\
\text { B } \\
300\end{array}$ & $\begin{array}{l}\mathrm{MH} \\
\mathrm{B}\end{array}$ & \begin{tabular}{|l|}
$\mathrm{MH}$ \\
$\mathrm{B}$ \\
150
\end{tabular} & $\begin{array}{l}\text { MH } \\
\text { B } \\
300\end{array}$ & $\begin{array}{l}\mathrm{MH} \\
\mathrm{B}\end{array}$ & \begin{tabular}{|l}
$\mathrm{MH}$ \\
$\mathrm{B}$ \\
150
\end{tabular} & $\begin{array}{l}\text { MH } \\
\text { B } \\
300\end{array}$ & $\begin{array}{l}\mathrm{MH} \\
\mathrm{B}\end{array}$ & $\begin{array}{l}\mathrm{MH} \\
\mathrm{B} \\
150\end{array}$ & $\begin{array}{l}\text { MH } \\
\text { B } \\
300\end{array}$ \\
\hline RRW12 & 10 & 4 & 8 & 4 & 2 & 8 & 4 & 4 & 8 & 4 & 4 & 8 \\
\hline $\begin{array}{l}\text { KKW1 } \\
2\end{array}$ & 8 & 2 & 8 & 4 & 2 & 8 & 4 & 4 & 8 & 2 & 4 & 8 \\
\hline RRL12 & 4 & 2 & 8 & 4 & 1 & 4 & 4 & 2 & 4 & 4 & 2 & 4 \\
\hline RR12 & 4 & 8 & 2 & 4 & 4 & 2 & 4 & 8 & 2 & 10 & 4 & 2 \\
\hline
\end{tabular}


The designed peptides were also found to be active against all Gram positive bacteria tested. Indeed all the peptides show either improved or unaltered activity in the presence of salt (Table 3.4-3). RR12 peptide show improved activity against all Gram positive strains in the presence of $300 \mathrm{mM} \mathrm{NaCl}$ concentration. Also in case of RRL12 peptide (where Trp residue in hydrophobic face) shows improved activity when compared to RRW12 (where Trp residue in cationic face) in the presence or absence of salt (Table 3.4-3).

SAR studies of buforin II peptide and its truncated analogs have revealed that RLLR motif in C-terminal of buforin II is responsible for antimicrobial and helix forming activity [43]. Hence it is conceived that the presence of RLLR residues at C-terminal of designed peptides have imparted enhanced antimicrobial activity and salt resistance may have stemmed from the appropriate combination of Arg, Trp and Ileu residues.

\subsubsection{Biophysical characterization:}

\subsubsection{Outer membrane permeability:}

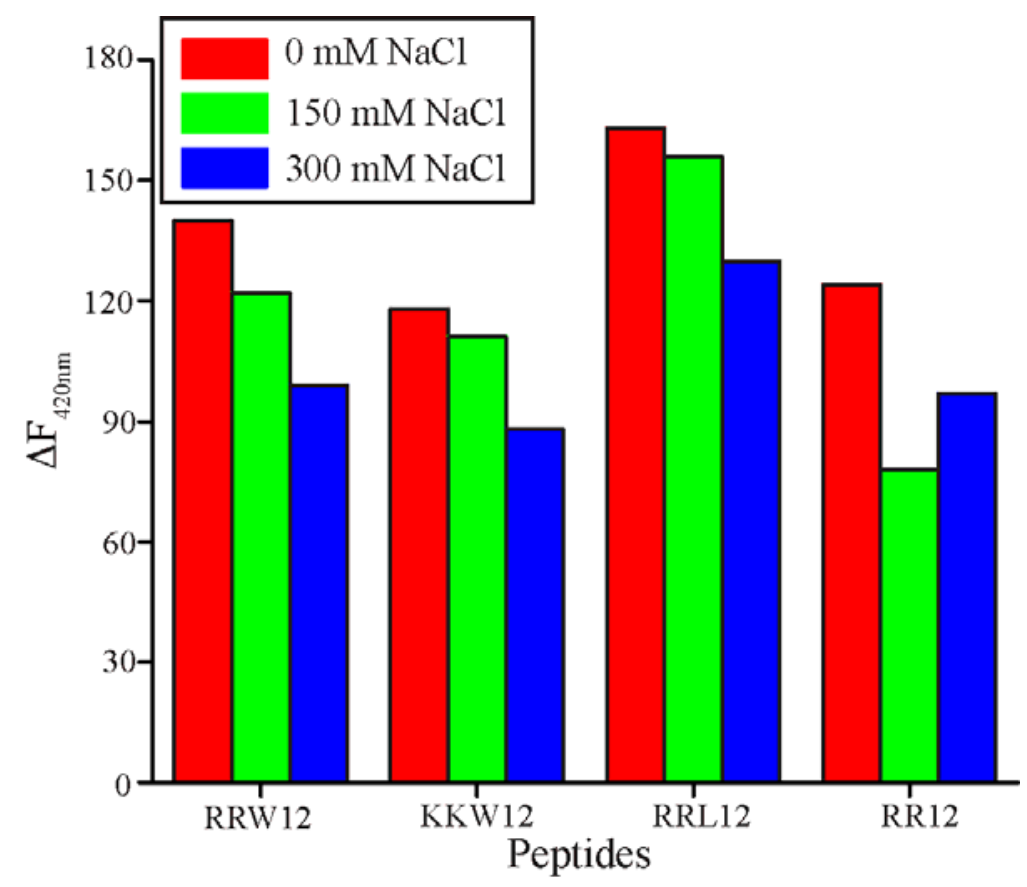

Figure 3.4-2: Outer membrane permeability of mid log phase grown E.coli cells using hydrophobic NPN dye in the absence of salt (red bars) and in the presence 


\section{of $150 \mathrm{mM} \mathrm{NaCl}$ (green bars), $300 \mathrm{mM} \mathrm{NaCl}$ (blue bars) by $10 \mu \mathrm{M}$ of designed peptides.}

The membrane integrity of bacteria has been found to be an important factor in the survival of bacteria and hence the designed peptides were first tested for the outer membrane permeabilizing ability. This activity was even tested in the presence of salt by growing E.coli to mid log phase in the presence of $150 \mathrm{mM}$ and $300 \mathrm{mM} \mathrm{NaCl}$. All of the peptides show dramatic increase in fluorescence intensity when added in increasing concentrations to E.coli cells in the absence of salt (Figure 3.4-2). In the presence of $\mathrm{NaCl}$, even though there was decrease in intensity when compared to

intensity without salt, all of the peptides show increase in fluorescence intensity with increase in peptide concentrations. At $10 \mu \mathrm{M}$ peptide concentrations, the peptide RR12 show improved permeabilizing ability in the presence of $300 \mathrm{mM} \mathrm{NaCl}$ when compared to $150 \mathrm{mM} \mathrm{NaCl}$ (Figure 3.4-2). For all the other peptides, at $10 \mu \mathrm{M}$ peptide concentrations there was decrease in intensity as the concentration of salt increases (Figure 3.4-2).

\subsubsection{Inner membrane permeability:}

The complete membrane disrupting ability of the designed peptides were tested with the help of cationic dye SYTOX green that fluoresces only when it binds to nucleic acids. Mid log phase grown E.coli cells were titrated with increasing concentration of peptides and the fluorescent intensity changes at $520 \mathrm{~nm}$ was recorded. Since the dye proved to be salt sensitive, the experiment was carried out in $10 \mathrm{mM}$ Phosphate buffer with $150 \mathrm{mM} \mathrm{NaCl}$.

All of the designed peptides show increase in intensity signal when the concentration of peptide increases. Among all of the peptides, KKW12 showed highest increase followed by RRW12, RR12 and RRL12 (Figure 3.4-3). 


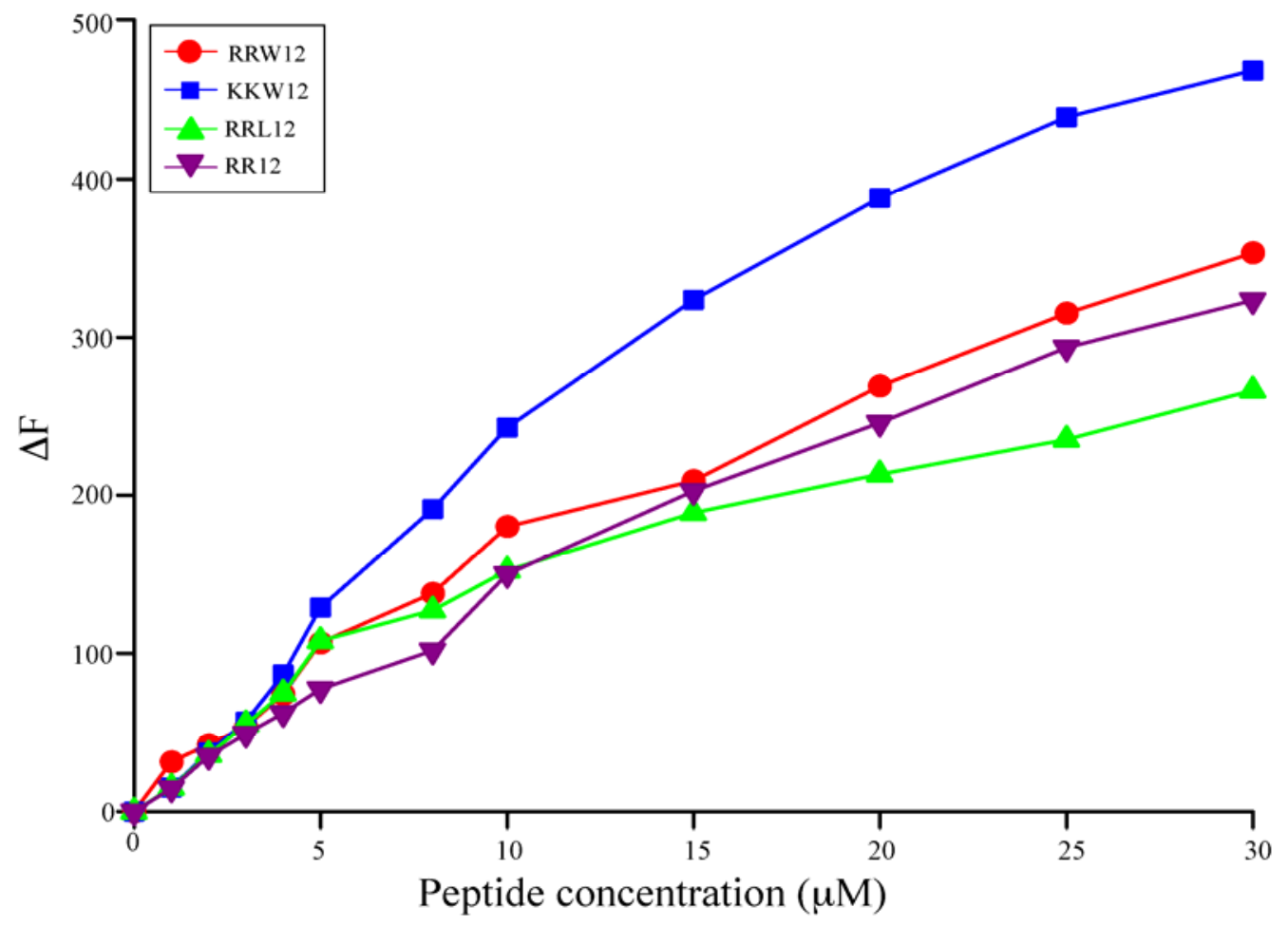

Figure 3.4-3: Inner membrane permeability of mid log phase grown E.coli cells using cationic SYTOX green dye of RRW12 (red), KKW12 (blue), RRL12 (green) and RR12 (purple) peptides.

\subsubsection{Liposome leakage assay:}

Liposomes are the accomplished mimics of membrane and rupture and release of dyes entrapped in liposomes are comparable to the membrane permeability of an intact bacterial cell. Since the interaction of designed peptides with outer membrane of Gram negative bacteria is highly emphasized in this study, liposomes were prepared with POPC and LPS in 80:20 ratio in the presence of $300 \mathrm{mM}$ or absence of $\mathrm{NaCl}$. 


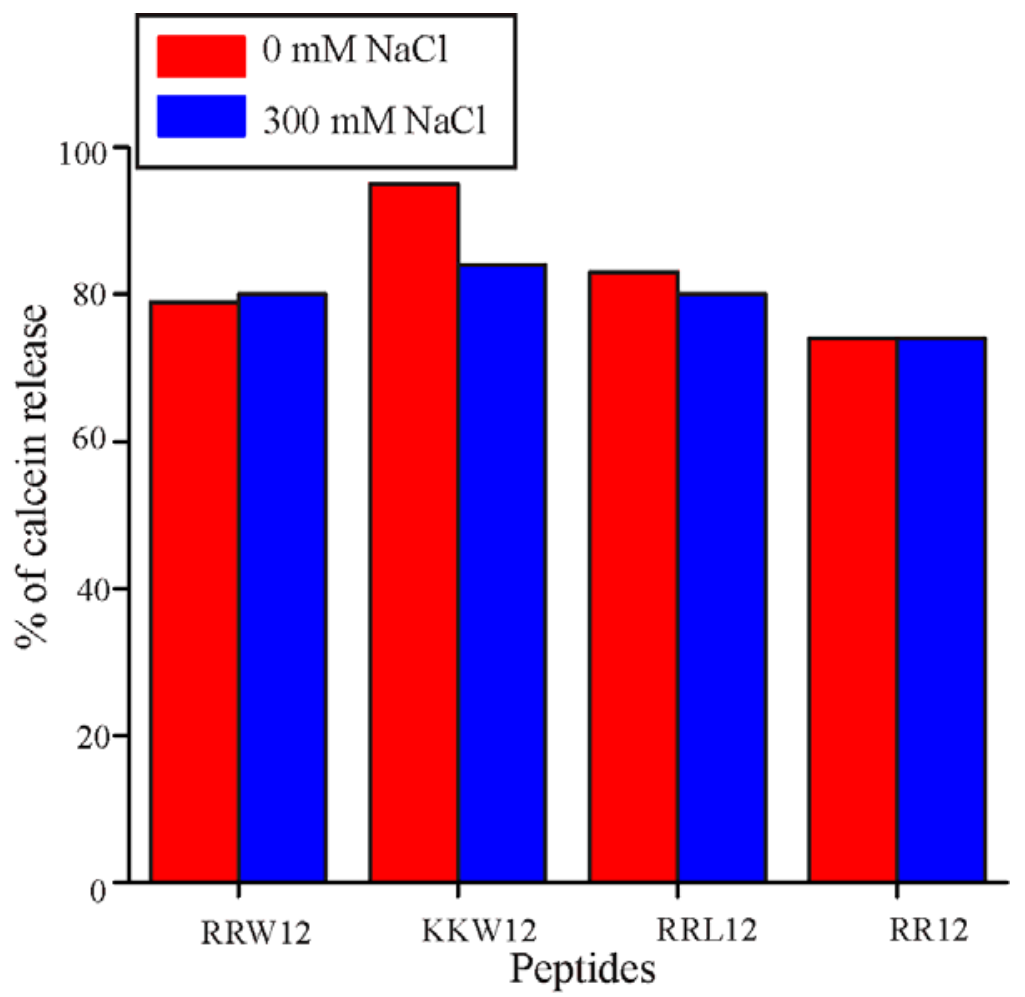

Figure 3.4-4: Leakage of calcein from POPC:LPS LUVs titrated with $10 \mu \mathrm{M}$ concentrations of designed peptides in $10 \mathrm{mM}$ phosphate buffer supplemented with no (red bars) or $300 \mathrm{mM} \mathrm{NaCl}$ (blue bars).

As the concentration of peptide increases, there was progressive release of trapped calcein from the LUVs. For RRW12 and RR12 peptides, at $10 \mu \mathrm{M}$ concentrations, there was $80 \%$ of leakage that did not change even in the presence of $\mathrm{NaCl}$ (Figure 3.4-4). On the other hand, for KKW12 and RRL12 peptides, there was slight decrease in leakage (Figure 3.4-4).

\subsubsection{Dissociation of FITC-LPS aggregates:}

When LPS is dispensed in aqueous buffer, it associates itself into aggregates forming micelles. In a typical LPS micelle, hydrophilic head is made up of O-antigen and core polysaccharides with hydrophobic lipid A core. LPS micelles are hence negatively charged because of the presence of carboxylate groups in head and phosphate group in lipid A. In the case of FITC-LPS, FITC molecules are entrapped in LPS micelles and hence quenched. When the designed peptides were added in increasing concentrations to FITC-LPS, they were attracted through ionic interactions followed by hydrophobic 
interactions resulting in the dissociation of LPS micelles. This results in dequenching of FITC fluorescence.

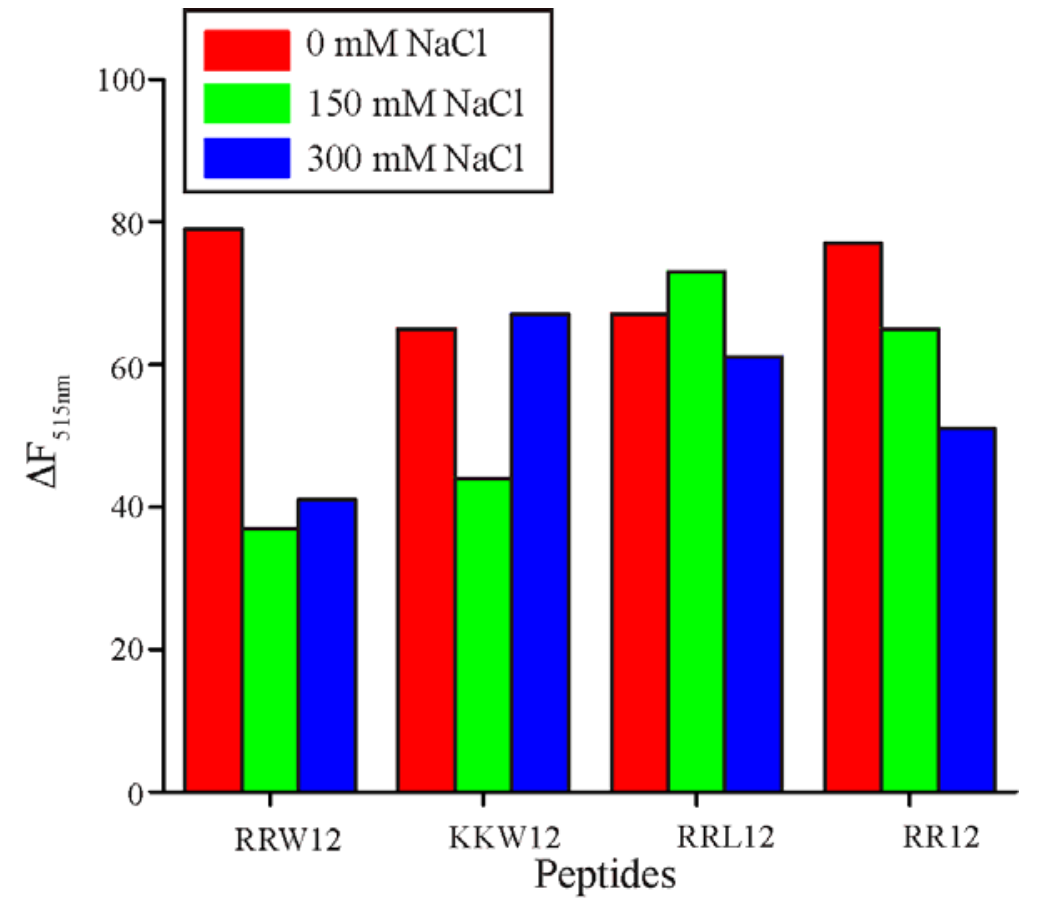

Figure 3.4-5: Dissociation of FITC-LPS aggregates in the absence of $\mathrm{NaCl}$ (red bars) and in the presence of $150 \mathrm{mM} \mathrm{NaCl}$ (green bars), $300 \mathrm{mM} \mathrm{NaCl}$ (blue bars) by $10 \mu \mathrm{M}$ concentrations designed peptides.

RRW12 and RR12 peptides show remarkable decrease in dissociating abiliy with increase in $\mathrm{NaCl}$ concentration (Figure 3.4-5 C). At $10 \mu \mathrm{M}$ peptide concentration, KKW12 show higher dissociation of LPS micelles in $300 \mathrm{mM} \mathrm{NaCl}$ when compared to $150 \mathrm{mM} \mathrm{NaCl}$ (Figure 3.4-5). 


\subsubsection{Isothermal titration calorimetry:}
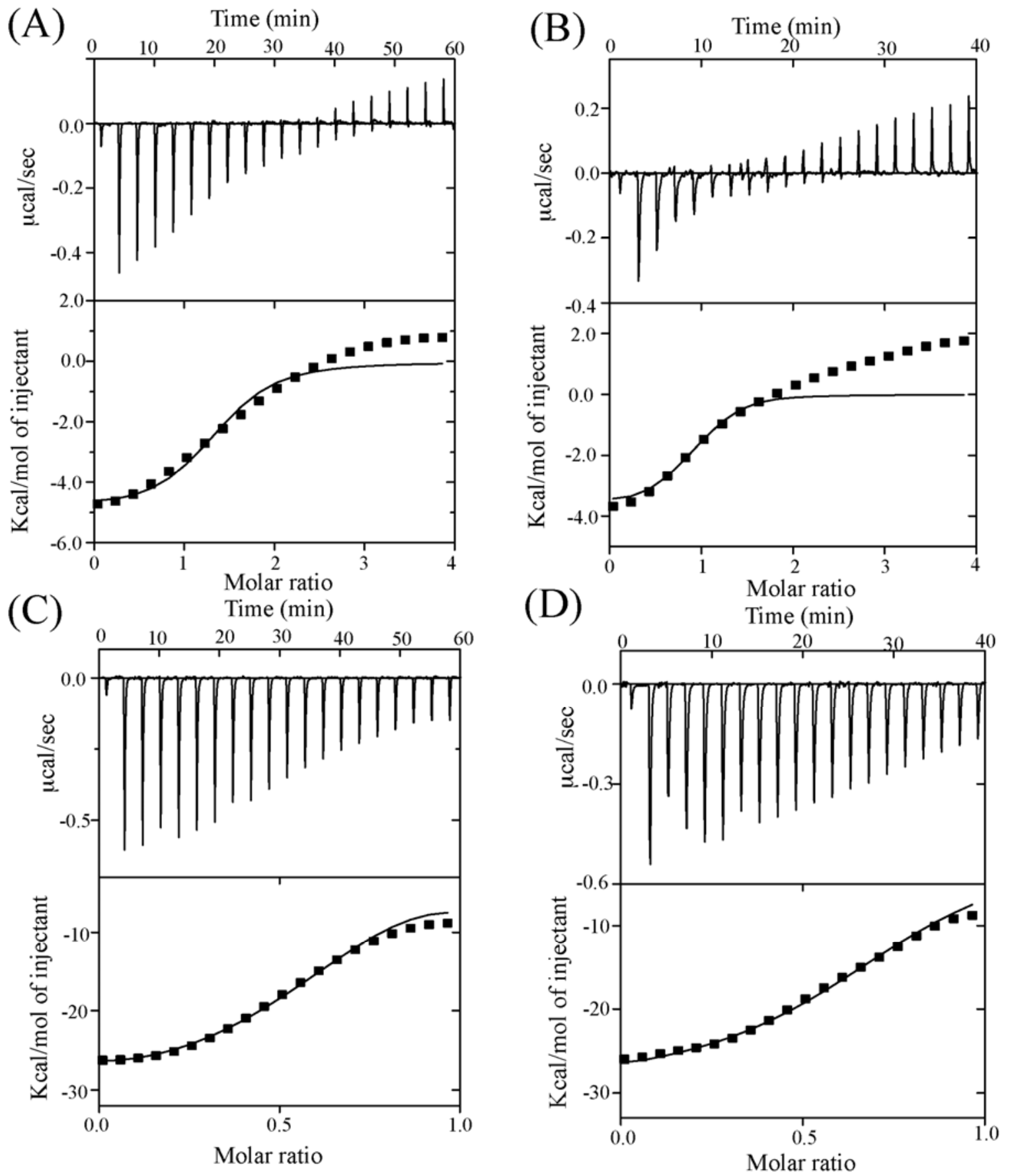

Figure 3.4-6: ITC thermograms of (A) RRW12, (B) KKW12, (C) RRL12 and (D) RR12 with LPS micelles at $25^{\circ} \mathrm{C}$.

The binding parameters for designed peptides with LPS micelles were determined using isothermal titration calorimetry at $25^{\circ} \mathrm{C}$. The binding reaction was found to be 
exothermic as revealed with downward peaks arising from each injection of peptide into LPS micelles (Figure 3.4-6). This discloses the dominance of ionic interactions because of the presence of more number of Arg residues.

Table 3.4-4: Calculation of thermodynamic parameters for the binding of designed peptides with LPS micelles at $25^{\circ} \mathrm{C}$ in the absence of salt.

\begin{tabular}{|c|c|c|c|c|}
\hline & RRW12 & KKW12 & RRL12 & RR12 \\
\hline $\mathrm{K}_{\mathrm{a}}\left(\mu \mathrm{M}^{-1}\right)$ & 0.53 & 1.18 & 0.44 & 0.65 \\
\hline$\Delta \mathrm{H}\left(\mathrm{kcal} . \mathrm{mol}^{-1)}\right.$ & -4.9 & -3.6 & -2.9 & -2.9 \\
\hline $\mathrm{T} \Delta \mathrm{S}\left(\mathrm{kcal}^{\mathrm{mol}} \mathrm{l}^{-1} \mathrm{deg}^{-1}\right)$ & 2.9 & 4.6 & 4.8 & 5.0 \\
\hline$\Delta \mathrm{G}\left(\mathrm{kcal} . \mathrm{mol}^{-1}\right)$ & -7.8 & -8.2 & -7.7 & -7.9 \\
\hline $\mathrm{K}_{\mathrm{d}}(\mu \mathrm{M})$ & 1.8 & 0.8 & 2.2 & 1.5 \\
\hline
\end{tabular}

The binding constants were derived from the fitting of injection points to one set of binding sites. RRW12 peptide saturates LPS micelles at about $15^{\text {th }}$ injection (Figure 3.4-6, A). On the other hand, KKW12 peptide brings about the saturation at around $5^{\text {th }}$ injection itself (Figure 3.4-6, B). The upward projection of peaks indicates the presence of extra peptide after complete saturation of LPS. The dissociation constant $\left(\mathrm{K}_{\mathrm{d}}\right)$ values represent the extent of binding. The binding of all of the peptides with LPS micelles seems to be very tight as revealed by low $\mathrm{K}_{\mathrm{d}}$ values (Table 3.4-4).

\subsubsection{Intrinsic tryptophan fluorescence:}

Tryptophan serves as an excellent intrinsic fluorescent probe to detect the localization of peptide in aqueous and micelle environment. In the absence of LPS micelles and $\mathrm{NaCl}$, the intrinsic fluorescene of tryptophan was found to be 360-354 nm indicating maximum exposure (Table 3.4-5) (Figure 3.4-7). However in the presence of $\mathrm{NaCl}$ the exposure of tryptophan residue slightly increased to $362-354 \mathrm{~nm}$. 
(A)
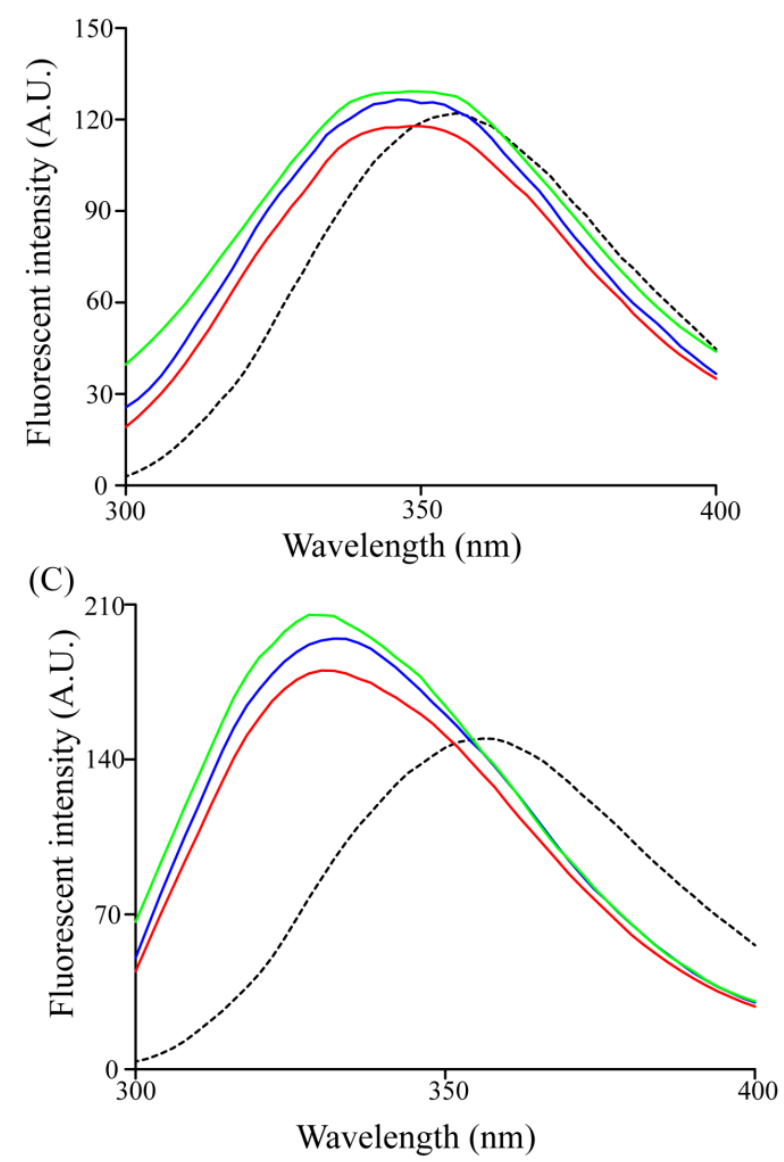

(B)

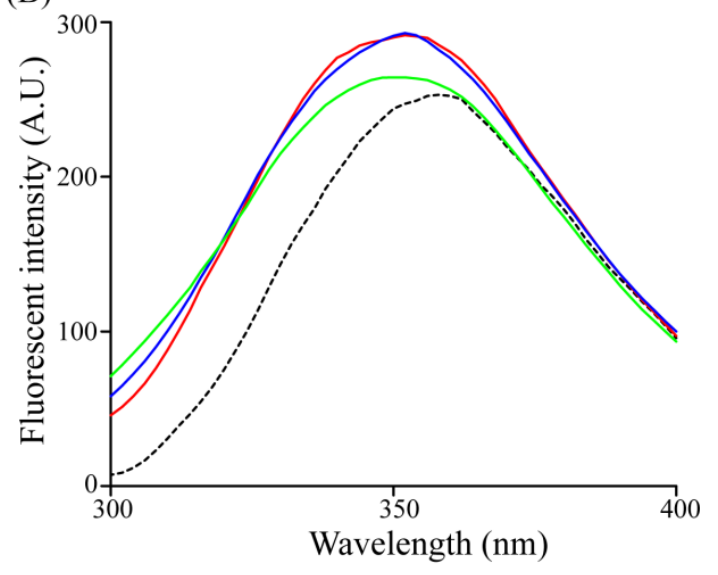

Figure 3.4-7: Intrinsic tryptophan fluorescence measurement of designed peptides (A) RRW12, (B) KKW12 and (C) RRL12, in the absence (black dotted lines) and presence of $3 \mu \mathrm{M}$ (red), $5 \mu \mathrm{M}$ (blue) and $10 \mu \mathrm{M}$ (green) LPS micelles in $10 \mathrm{mM} \mathrm{PO}_{4}$ buffer.

Table 3.4-5: Emission maximum of Trp fluorescence ( $\lambda$ max) of peptides, in the aqueous environment (0 LPS) and in LPS micelles in $10 \mathrm{mM} \mathrm{PO}_{4}$ buffer alone and also in the presence of $150 \mathrm{mM}$ and $300 \mathrm{mM} \mathrm{NaCl}$.

\begin{tabular}{|l|l|l|r|l|l|l|}
\hline \multirow{2}{*}{} & \multicolumn{4}{l}{$\lambda \max (0$ LPS $)$} & \multicolumn{2}{l|}{$\lambda \max (20 \mu \mathrm{M}$ LPS $)$} \\
\cline { 2 - 8 } & $\mathrm{mM}$ & $1 . \mathrm{m}$ & $\tilde{\mathrm{m}}$ & $\mathrm{mM}$ & $1 . \mathrm{m}$ & $\mathrm{m}$ \\
\hline RRW12 & 354 & 356 & 356 & 346 & 348 & 348 \\
\hline KKW12 & 360 & 360 & 362 & 340 & 358 & 354 \\
\hline RRL12 & 354 & 352 & 358 & 326 & 326 & 322 \\
\hline
\end{tabular}


When LPS was added in increasing concentrations, emission maxima of tryptophan experienced a blue shift towards shorter wavelengths (Table 3.4-5) (Figure 3.4-7). This indicates the insertion of peptides into hydrophobic environment. RRL12 peptide where Trp residue is in hydrophobic face, experienced a greater blue shift of about 322-326 nm (Figure 3.4-7, C).

\subsubsection{Acrylamide quenching:}

Acrylamide is a neutral quencher and quenches the fluorescence of tryptophan when it is exposed. The extent of exposure of Trp residue can be assessed using Stern-Volmer constant $\left(\mathrm{K}_{\mathrm{sv}}\right)$.

Table 3.4-6: Quenching of tryptophan residue of the peptides in free and in LPS micelles by acrylamide in $10 \mathrm{mM} \mathrm{PO}_{4}$ buffer alone and also in the presence of 150 $\mathrm{mM}$ and $300 \mathrm{mM} \mathrm{NaCl}$.

\begin{tabular}{|l|l|l|l|l|l|l|}
\hline \multirow{2}{*}{} & \multicolumn{2}{|l|}{$\mathrm{K}_{\text {sv }}$ (free) } & \multicolumn{2}{l|}{$\mathrm{K}_{\text {sv }}($ LPS $)$} \\
\cline { 2 - 8 } & $\mathrm{mM}$ & $1 . \mathrm{m}$ & $\mathrm{m}$ & $\mathrm{mM}$ & $1 . \mathrm{m}$ & $\mathrm{m}$ \\
\hline RRW12 & 27.9 & 17.6 & 17.5 & 6.3 & 7.2 & 7.5 \\
\hline KKW12 & 23.6 & 18.0 & 19.8 & 7.5 & 5.6 & 5.8 \\
\hline RRL12 & 27.4 & 30.2 & 24.1 & 4.7 & 5.2 & 5.6 \\
\hline
\end{tabular}

As can be seen, the $\mathrm{K}_{\mathrm{sv}}$ values of peptides in the absence of LPS micelles was very high when compared to the $\mathrm{K}_{\mathrm{sv}}$ values in the presence of LPS micelles (Table 3.4-6). This further proves the incorporation of peptides into hydrophobic environment of LPS. As a result of which, the Trp residue would be buried inside and unavailable for the quencher to quench its fluorescence. 


\subsubsection{Circular Dichroism spectroscopy:}
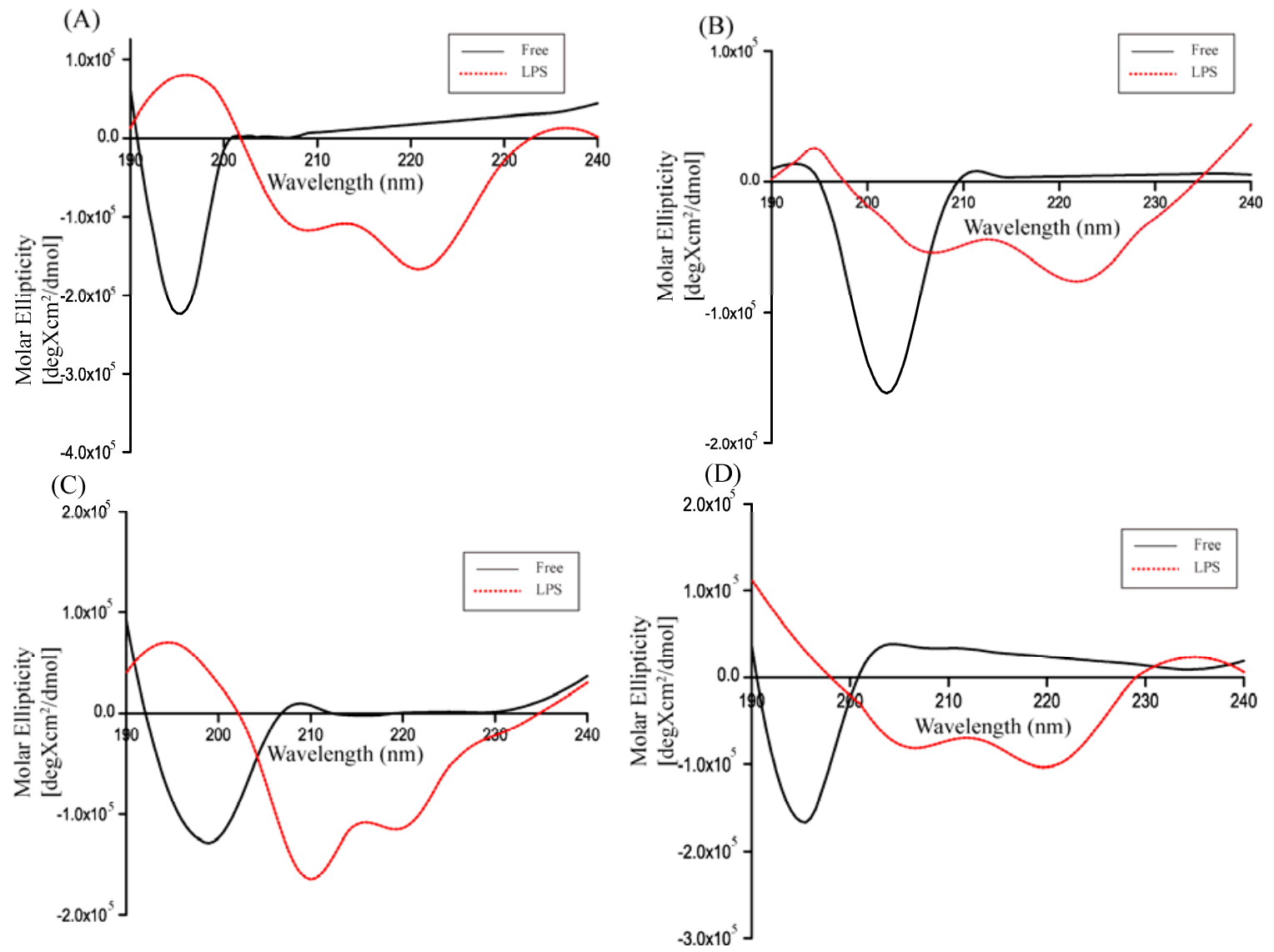

Figure 3.4-8: Far UV CD measurements of (A) RRW12, (B) KKW12, (C) RRL12 and (D) RR12 in the aqueous state (black) and in the presence of LPS (red dots).

The global fold of peptides in the presence of LPS micelles was determined using Far UV CD measurements. In aqueous state, all of the peptides exhibited minima around $195 \mathrm{~nm}$ (Figure 3.4-8). This peak is characteristic of random coil conformation. This explains that the peptides do not fold into specific conformation in buffer and exist as random coils. Addition of $30 \mu \mathrm{M}$ of LPS to the peptides brings about change in its CD profile. Presence of two minima at $208 \mathrm{~nm}$ and $222 \mathrm{~nm}$ and maxima around $195 \mathrm{~nm}$ are indicative of helical conformations (Figure 3.4-8).

\subsubsection{Discussion:}

In order to develop a pharmaceutically active antimicrobial peptide, it has to be active at physiological salt concentration. Even though the antimicrobial peptides adopt 
variety of conformations from alpha helical, beta sheets, beta hairpins to extended structures, most of the antimicrobial peptides that are membrane active are found to be salt susceptible [182]. Despite its broad spectrum of activity, Human $\beta$-defensin 1 was found to be salt sensitive in cystic fibrosis patients [188]. Research on the development of salt resistant antimicrobial peptides accounts for more than a decade. Recent developments include dimerization of broad spectrum designed AMP [189], replacement of aminoacid residues with bulky aminoacid $\beta$-naphthylalanine [142] , addition of helix stabilizing motifs [190] or engineer salt resistant motif from ribosomally synthesized AMPs to synthetic peptides [31]. In all of these developments, design of peptides was targeted to provide a larger hydrophobic surface than their primitive parent peptides. It is for the same reason that the peptides which are dimerized or oligomerized with one or more disulfide linkages conferred salt resistance as in the case of protegrins [34] and bactenecins [183].

Apart from these changes, it was also noted that simple replacements of aminoacid residues of a broad spectrum active AMP can result in considerable change in their salt susceptibility. Studies on a library of peptides based on Trp rich antimicrobial peptide PEM-2 revealed that a simple mutation of $\mathrm{W} 5 \mathrm{~K} / \mathrm{A} 9 \mathrm{~W}$ has rendered the peptide salt resistant [115]. However increase in hydrophobicity increases hemolytic activity i.e. it abolishes cell selectivity. It has been found that such problem can be overcome by the addition of more positive charges in hydrophilic side of the peptide [115].

In this aspect, peptides with appropriately placed Arg, Leu and Trp residues in helical wheel were designed and simple replacements were also carried out to generate a series of salt resistant antimicrobial peptides. All of the designed peptides, RRW12, KKW12, RRL12 and RR12 were found to exhibit broad spectrum of activity against all of the pathogens tested. Interestingly, these peptides were found to be salt resistant showing unaltered or negligible changes in bacterial killing activity. These peptides, irrespective of its arrangement of residues, exhibited excellent membrane disrupting activity also. In the context of LPS, all of the designed salt resistant AMPs showed satisfactory perturbation of LPS micelles. Isothermal titration calorimetry results 
indicate that the peptides bind to LPS very tightly and the reactions were exothermic. The global fold of the peptides in the presence of LPS was found to be alpha helical. Collectively these results suggest that the aminoacid residues in the salt resistant AMPs are aptly knitted to form helical pattern. Arg and Trp residues are placed in such a manner that they involve in cation-pi interaction. The much needed larger hydrophobic surface for salt resistance would then be generated with the cation-pi interaction with the surrounding Leu and Ile residues. 


\subsection{Conclusion summary}

Development of antibiotic resistance has become a widespread threat than any other infectious diseases and the pace at which bad bugs that are resistant to broad range of drugs developing is alarming the researchers to generate new antimicrobials. Antimicrobial availability task force (AATF) has identified Acinetobacter baumannii, beta lactamase producing Enterobacteriaceae and methicillin resistant Staphylococcus aureus as some of the bad bugs [191]. Among the new drugs that are under clinical trials, majority of the drugs are found to be active against gram positive bacteria and very few drugs derived from cephalosporins and carbapenems are active against gram negative bacilli [191]. Lipopolysaccharides (LPS), an integral glycolipid present in the outer layer of outer membrane of gram negative bacteria are found to play an important role in determining the permeability and activity of hydrophobic compounds [69]. Eventhough LPS exists as bilayer structure in outer membrane, the higher order structure of lipidA provides rigidity to the membrane just as cholesterol in mammalian membranes [70-73]. Apart from this permeability hinderance, LPS also induces inflammatory responses that leads to septic shock [64].

The identification of cationic antimicrobial peptides (AMPs) to exhibit broad spectrum of antimicrobial activity shed light on the development of agents that can provide alternative to antibiotics. AMPs from natural origin also serve as template for the synthesis of effective antimicrobials targeted towards specific organism also [14]. Not all antimicrobial peptides can neutralize free LPS in the blood stream. Hence special attention is needed towards the development of antimicrobial antiendotoxic agent. Towards such improvement, we developed $\beta$-boomerang peptides (YI12) that are found to exhibit efficient antimicrobial and antiendotoxic activity. Structureactivity correlation studies of YI12 peptides also revealed a structured LPS binding motif (WKRKRF) [150]. Among the YI12 peptides studied, YI12WF exhibited more efficient antibacterial and antiendotoxic activity. Atomic resolution studies of YI12WF revealed an amphipathic conformation of the peptide upon membrane interaction that resolves amphiphilic cationic residues on one side and hydrophobic 
aromatic lock formed of W4-F9 on the other side [150]. YI12WF peptide was further characterized by dimerization and lipidation.

Cysteine analogs of YI13WFC showed promised increase in antibacterial and LPS neutralization activity. Among the different acyl chain lengths tested, $\mathrm{C} 4$ chain was found to be optimum for YI13WFC. Atomic resolution structure of C4YI13WFC in aqueous media displayed conserved amphipathic structure with aromatic Trp-Phe lock. It is envisioned that the presence of disulphide bridge folds the peptide in such a way that all of the polar residues are segregated on one side with W4-F12 aromatic lock on the other side. When this peptide was titrated with different concentrations of LPS, resonances arising from the interaction of sugar moieties of lipidA with F12 residue were clearly seen. Thus it is concluded that the dimeric cationic solvent exposed residues identify the negatively charged LPS layer and the hydrophobic aromatic lock brings about the disintegration of LPS molecules. The presence of acylation in N-terminus further stabilizes this interaction.

SAR studies of the hybrid peptides LG21, FG21, KG20 and LG21R19A shed important insights in LPS identification and detoxification at the molecular level of those peptides aggregating on LPS layer. The structure of hybrid peptide LG21 in DPC micelles revealed alpha helical conformation with cation-pi interaction between W15 and R19. When R19 was mutated to Ala, the cation-pi interaction was lost with loss of activity. Hence it is conceived that the basic residues in the $\beta$-boomerang part gets electrostatically attracted towards the negatively charged LPS followed with the outer membrane and inner membrane disruption by leucine side chains of temporin part in N-terminal.

Structure-activity correlation studies on cysteine deleted analogs of PG-1 and salt resistant alpha helical peptides revealed the importance of arginine and lysine residues in imparting the ionic interactions between the peptides and outer membrane of gram negative bacteria. Overall, the peptides designed in this work provide valuable insights towards the development of antibacterial antiendotoxic agents. 


\subsection{Future perspectives:}

The antimicrobial peptides designed in this study can be utilized for further modifications like inclusion of non natural aminoacids or addition of d-enantiomeric forms of aminoacids to bring about protease resistant activity. The hybrid peptides and salt resistant peptides can be further analysed for serum resistant activity and in vivo septic shock models. 


\section{Appendix 1:}

List of unambiguously assigned NOEs from RR14 and RR11 tr-NOESY spectra in LPS at $298 \mathrm{~K}$ and pH 4.5.

\begin{tabular}{|c|c|c|c|}
\hline & RR14 & & RR11 \\
\hline $\begin{array}{l}12 \text { VAL QG11 } \\
12 \text { VAL QG21 } \\
11 \text { VAL HB } \\
14 \text { ARG QB } \\
14 \text { ARG QG } \\
4 \text { ARG QG } \\
4 \text { ARG QB } \\
10 \text { PHE QB } \\
10 \text { PHE QB } \\
10 \text { PHE HA } \\
10 \text { PHE QD } \\
10 \text { PHE QD }\end{array}$ & $\begin{array}{l}4 \text { ARG HA } \\
4 \text { ARG HA } \\
5 \text { LEU HA } \\
3 \text { GLY } \\
3 \text { GLY H } \\
13 \text { GLY HA } \\
13 \text { GLY HA } \\
6 \text { TYR QD } \\
6 \text { TYR QE } \\
6 \text { TYR H } \\
6 \text { TYR QD } \\
6 \text { TYR QE }\end{array}$ & $\begin{array}{l}5 \text { ARG QG } \\
6 \text { ARG QG } \\
5 \text { ARG QB } \\
6 \text { ARG QB } \\
5 \text { ARG QD } \\
11 \text { ARG HA } \\
3 \text { TYR HA } \\
4 \text { ARG HA } \\
9 \text { VAL HG21 } \\
9 \text { VAL HG11 } \\
9 \text { VAL HG21 } \\
9 \text { VAL HG11 } \\
4 \text { ARG QB } \\
9 \text { VAL HG21 } \\
9 \text { VAL HG21 } \\
9 \text { VAL HG21 } \\
9 \text { VAL HG11 } \\
9 \text { VAL HG11 } \\
9 \text { VAL HG11 }\end{array}$ & $\begin{array}{l}3 \text { TYR QD } \\
3 \text { TYR QD } \\
3 \text { TYR QE } \\
3 \text { TYR QE } \\
3 \text { TYR QE } \\
3 \text { TYR H } \\
11 \text { ARG H } \\
8 \text { VAL H } \\
3 \text { TYR QE } \\
3 \text { TYR QD } \\
3 \text { TYR H } \\
3 \text { TYR H } \\
8 \text { VAL H } \\
7 \text { PHE QD } \\
7 \text { PHE QZ } \\
7 \text { PHE QE } \\
7 \text { PHE QD } \\
7 \text { PHE QZ } \\
7 \text { PHE QE }\end{array}$ \\
\hline
\end{tabular}




\section{BIBLIOGRAPHY}

1. Davies, J. and Davies, D. Origins and Evolution of antibiotic resistance. Microbiology and Molecular Biology Reviews, 2010. 74: 417-433.

2. Abraham, E.P. and Chain, E. An enzyme from bacteria able to destroy penicillin. Reviews of infectious diseases, 1940. 10: 677-678.

3. Vollimer, S. RTP panels address rogues gallery of multidrug-resistant bacteria, in Science in the triangle 2011: North Carolina.

4. Boucher, H.W., Talbot, H.G., Benjamin jr, K.D., Bradley, J., Guidos, R.J., Jones, R.N., Murray, B.E., Bonomo, R.A., Gilbert, D. $10 X 20$ Progressdevelopment of new drugs active against Gram negative Bacilli: an update from the infectious diseases society of America. Clinical Infectious Diseases, 2013. 56: $1685-1694$.

5. Freiden, D.T. Threat report 2013, Centers for disease control and prevention: Atlanta.

6. Andersson, D.I. and Hughes, D. Antibiotic resistance and its cost: is it possibe to reverse resistance? Nature Reviews Microbiology, 2010. 8 : 260.

7. Neu, H.C. Overview of mechanisms of bacterial resistance. Diagnositc Microbiology and Infectious disease, 1989. 12: p. 109-116.

8. Ganz, T. and Lehrer, R.I. Antimicrobial peptides of vertebrates. Current opinion in Immunology, 1998. 10: p. 41-44.

9. Fennell, J.F., SHipman, W.H. and Cole, L.J. Antibacterial action of a bee venom fraction (Melittin) against a penicillin-resistant Staphylococcus and other microorganisms. 1967.

10. Steiner, H., Hultmark, D., Engstrom, A., Bennich, H. and Boman, H.G. Sequence and specificity of two antibacterial proteins involved in insect immunity. Nature, 1981. 292: p. 246-248.

11. Lehrer, R.I., Lichtenstein, A.K. and Ganz, T. Defensins: Antimicrobial and cytotoxic peptides of mammlian cells. Annual review of Immunology, 1993. 11: p. $105-128$.

12. Zasloff, M. Magainins, a class of antimicrobial peptides from Xenopus skin: Isolation, characterization of two active forms and partial cDNA sequence of a 
precursor. Proceedings of National Academy of Sciences, 1987. 84: p. 54495453.

13. Hancock, R.E.W. and Lehrer, R.I. Cationic peptides: a new source of antibiotics. Trends in Biotechnology, 1998. 16: p. 82-88.

14. Nguyen, L.T., Haney, E.F. and Vogel, H.J. The expanding scope of antimicrobial peptide structures and their modes of action. Trends in Biotechnology, 2011. 9: p. 464-472.

15. Marcos, J.F., Munoz, A., Perez-Paya, E., Misra, S. and Lopez-Garcia B, Identification and rational design of novel antimicrobial peptides for plant protection. The Annual review of Phytopathology, 2008. 46: p. 273-301.

16. Jenssen, H., Hamill, P. and Hancock, R.E.W. Peptide antimicrobial agents. Clincial Microbiology Reviews, 2006. 19: p. 491-511.

17. Cezard, C., Silva Pires, V., Mullie, C., and Sonnet, P. Antibacterial peptides: A review, in Science against microbial pathogens:communicating current research adn technological advances, A.Mendez-Vilas, Editor 2011, Formatex research center: Spain. p. 926-937.

18. Tossi, A., Sandri, L. and Giangaspero A. Amphipathic alpha helical antimicrobial peptides. Biopolymers (Peptide Science), 2000. 55: p. 4-30.

19. Wieprecht, T., Apostovlov, O., Beyermann, M. and Seelig J. Thermodynamics of the alpha helix-coil transition of amphipathic peptides in a membrane environment: implications for the peptide-membrane binding equilibrium. Journal of Molecular Biology, 1999. 294: p. 785-794.

20. Gesell, J., Zasloff, M. and Opella, S.J. Two-dimensional 1H NMR experiments show that the 23-residue magainin antibiotic peptide is an alpha helix in dodecylphosphocholine micelles, sodium dodecylsulfate micelles and trifluoroethanol/water solution. Journal of Biomolecular NMR, 1997. 9: p. 127135.

21. Matsuzaki, K. Magainins as paradigm for the mode of action of pore forming polypeptides. Biochimica et Biophysica Acta, 1998. 1376: p. 391-400. 
22. Matsuzaki, K. Why and how are peptide-lipid interactions utilized for self defense? Magainins and tachyplesins as archetypes. Biochimica et Biophysica Acta, 1999. 1462: p. 1-10.

23. Hultmark, D., Steiner, H., Rasmuson, T., and Boman, H.G. Insect immunity: Purification and properties of three inducible bactericidal protiens from hemolymph of immunized pupae of hyalophora cecropia. European journal of Biochemistry, 1980. 106: p. 7-16.

24. Inagaki, F., Shimada, I., Kawaguchi, K., Hirano, M., Tersawa, I., Ikura, T. and Go N. Structure of Melittin bound to perdeuterated dodecylphosphocholine micelles as studied by two-dimensional NMR and distance geometry calculations. Biochemistry, 1989. 28: p. 5985-5991.

25. Bhunia, A., Domadia, P.N. and Bhattacharjya, S. Structural and thermodynamic analyses of the interaction between melittin and lipopolysaccharide. Biochimical et biophysica Acta, 2007. 1768: p. 3282-3291.

26. Bhunia, A., Ramamoorthy, A. and Bhattacharjya, S. Helical Hairpin Structure of a Potent Antimicrobial Peptide MSI-594 in Lipopolysaccharide Micelles by NMR Spectroscopy. Chemistry-An European Journal, 2009. 15: p. 2036-2040.

27. Bhunia, A., Domadia, P.N., Torres, J., Hallock, K.J., Ramamoorthy, A. and Bhattacharjya S. NMR structure of Pardaxin, a pore forming antimicrobial peptide in lipopolysaccharide micelles. The Journal of Biological Chemistry, 2010. 285: p. 3883-3895.

28. Boman, H.G. Peptide antibiotics and their role in innate immunity. Annual reviews of Immunology, 1995. 13: p. 61-92.

29. Epand, R.M. and Vogel, H.J. Diversity of antimicrobial peptides and their mechanisms of action. Biochimica et Biophysica Acta, 1999. 1462: p. 11-28.

30. Bhattacharjya, S. De novo Designed Lipopolysaccharide Binding Peptides: Structure Based Development of Antiendotoxic and Antimicrobial Drugs. Current Medicinal Chemistry, 2010. 17: p. 3080-3093.

31. Saravanan, R., Xi, L., Lim, K., Mohanram, H., Peng, L., Mishra, B., Basu, A., Lee, J.M., Bhattacharjya, S. and Leong, S.S.J. Design of short membrane selective antimicorbial peptides containing tryptophan and arginine residues for 
improved activity, salt resistance and biocompatibility. Biotechnology and Bioengineering, 2013. 111: p. 37-49.

32. Saravanan, R., Mohanram, H., Joshi, M., Domadia, P.N., Torres, J., Ruedl, C. and Bhattacharjya, S. Structure, activity and interactions of the cysteine deleted analog of tachyplesin-1 with lipopolysaccharide micelle:Mechanistic insights into outer membrane permeabilization and endotoxin neutralization. Biochimica et Biophysica Acta, 2012. 1818: p. 1613-1624.

33. Ramamoorthy, A., Thennarasu, S., Tan, A., Gottipati, K., Sreekumar, S., Hey, D.L., An, F.Y.P., and Shelburne, C.E. Deletion of all cysteines in tachyplesin-1 abolishes hemolytic activity and retains antimicrobial activity and lipopolysaccharide selective binding. Biochemistry, 2006. 45: p. 6529-6540.

34. Harwig, S.S.L., Waring, A., Yang, H.J., Cho, Y., Tan, L. and Lehrer, R.I. Intramolecular disulfide bonds enhance the antimicrobial and lytic activities of protegrins at physiological sodium chloride concentrations. European journal of biochemistry, 1996. 240: p. 352-357.

35. Epand, R.M. and Vogel, H.J. Diversity of antimicrobial peptides and their mechanisms of action. Biochimica et Biophysica Acta, 1999. 1462: p. 11-28.

36. Fehlbaum, P., Bulet, P., Chernysh, S., Briand, J.P., Roussel, J.P., Letellier, L., Hetru, C. and Hoffmann, J.A. Structure-activity analysis of thanatin, a 21 residue inducible insect defense peptide with sequence homology to frog skin antimicrobial peptides. Proceedings of National Academy of Sciences, 1996. 93: p. 1221-1225.

37. Wallace, B.A. Structure of Gramicidin A. Biophysical Journal, 1986. 49: p. 295306.

38. Brogden, K.A. Antimicrobial peptides: Pore formers or metabolic inhibitors in bacteria? Nature Reviews Microbiology, 2005. 3: p. 250.

39. Sengupta, D., Leontiodou, H., Mark, A.E. and Marrink, S.J. Toroidal pores formed by antimicrobial peptides show significant disorder. Biochimica et Biophysica Acta, 2008. 1778: p. 2308-2317.

40. Shai, Y. Mode of action of membrane active antimicrobial peptides. Biopolymers (Peptide Science), 2002. 66: p. 236-248. 
41. Dong, N., Ma, Q., Shan, A., Lv, Y., Hu, W., Gu, Y., and Li, Y. Strand length dependent antimicrobial activity and membrane active mechanism of arginine and valine rich beta hairpin like antimicrobial peptides. Antimicrobial agents and chemotherapy, 2012. 56: p. 2994-3003.

42. Subbalakshmi, C. and Nagaraj, S. Mechanism of antimicrobial action of Indolicidin. FEMS Microbiology letters, 1998. 160: p. 91-96.

43. Park, C.B., Yi, K., Matsuzaki, K., Kim, M.S., and Kim, S.C. Structure-activity analysis of buforin-II, a histone H2-A derived antimicrobial peptide: The proling hinge is responsible for the cell penetrating ability of buforin-II. Proceedings of National Academy of Sciences, 2000. 97: p. 8245-8250.

44. Allende, D. and Mcintosh, T.J. Lipopolysaccharides in bacterial membranes act like cholesterol in eukaryotic plasma membranes in providing protection against Melittin-induced bilayer lysis. Biochemistry, 2003. 42: p. 1101-1108.

45. Verkleij, A.J., Zwaal, R.F.A., Roelofsen, B., Comfurius, P., Kastelijn, D.and van Deenen, L.L.M. The asymmetric distribution of phospholipids in the human red cell membrane. A combined study using phopholipases and freeze-etch electron microscopy. Biochimica et Biophysica Acta, 1973. 323: p. 178-193.

46. Brender, J.R., McHenry, A.J. and Ramamoorthy, A. Does Cholesterol play a role in the bacterial seletivity of antimicrobial peptides? Frontiers in Immunology, 2012. 3: p. 1-4.

47. Matasuzaki, K., Sugishta, K.I., Ishibe, N., Ueha, M., Nakata, S., Miyajima, K. and Epand,R.M. Relationship of membrane curvature to the formation of pores by Magainin 2. Biochemistry, 1998. 37: p. 11856-11863.

48. de Kruijff, B. Lipids beyond the bilayer. Nature, 1997. 386: p. 129-130.

49. Haney, E.F., Nathoo, S., Vogel, H.J. and Prenner, E.J. Induction of non-lamellar lipid phases by antimicrobial peptides: a potential link to mode of action. Chemistry and Physics of Lipids, 2010. 163: p. 82-93.

50. Epand, R.M., Shai, Y., Segrest, J.P. and Anantharamaiah, G.M. Mechanism for the modulation of membrane bilayer properties by amphipathic helical peptides. Biopolymers (Peptide Science), 1995. 37: p. 319-338. 
51. Hallock, K.J., Lee, D. and Ramamoorthy, A. MSI-78, an analogue of the magainin antimicrobial peptides disrupts lipid bilayer structure via positive curvature strain. Biophysical Journal, 2003. 84: p. 3052-3060.

52. Powers, J.P., Tan, A., Ramamoorthy, A. and Hancock, R.E.W. Solution structure and interaction of the antimicrobial polyphemusins with lipid membranes. Biochemistry, 2005. 44: p. 15504-15513.

53. Matsuzaki, K., Sugishita, K.I., Fujii, N. and Miyajuma,K. Molecular basis for membrane selectivity of an Antimicrobial peptide, Magainin 2. Biochemistry, 1995. 34: p. 3423-3429.

54. Oren, Z. and Shai, Y. From carpet mechanism to de-novo designed diastereomeric cell-selective antimicrobial peptides. Peptides, 2001. 22: p. 1629-1641.

55. Nunn, R.S. and Needham, D. Elastic deformation and failure of lipid bilayer membranes containing cholesterol. Biophysical Journal, 1990. 58: p. 997-1009.

56. Zasloff, M. Antimicrobial peptides of multicellular organisms. Nature, 2002. 415: p. 389-395.

57. Asthana, N. ,Yadav, S.P. and Ghosh, J.K. Dissection of antibacterial and toxic activity of Melittin: A leucine zipper motif plays a crucial role in determining its hemolytic activity but not antibacterial activity. The Journal of Biological Chemistry, 2004. 2004: p. 55042-55050.

58. Saravanan, R., Bhunia, A. and Bhattacharjya, S. Micelle-bound structures and dynamics of the hinge deleted analog of melittin and its diastereomer: Implications in cell selective lysis by D-amino acid containing antimicrobial peptides. biochimical et biophysica Acta, 2010. 1798: p. 128-319.

59. McInnes, C., Kondejewski, L.H., Hodges, R.S. and Sykes,B.D. Development of the structural basis for antimicrobial and hemolytic activities of peptides based on Gramicidin $S$ and design of novel analogs using NMR spectroscopy. The journal of Biological Chemistry, 2000. 275: p. 14287-14294.

60. Chen, Y., Guarnieri, M.T., Vasil, A.I., Vasil, M.L., Mant, C.T. and Hodges, R.S. Role of peptide hydrophobicity in the mechanism of action of alpha helical 
antimicrobial peptides. Antimicrobial agents and chemotherapy, 2007. 51: p. 1398-1406.

61. Wimley, W.C. Describing the mechanism of antimicrobial peptide action with the interfacial activity model. ACS Chemical Biology, 2010. 5(10): p. 905-917.

62. Mayers, D.L., Lerner, S.A., Ouellette,M. and Sobel,J.D. Antimicrobial drug resistance:Mechanisms of Drug resistance, D. L.Mayer, Editor 2009, Springer.

63. Bhattacharjya, S. De novo Designed Lipopolysaccharide Binding Peptides: Structure Based Development of Antiendotoxic and Antimicrobial Drugs. Current Medicinal Chemistry, 2010. 17(27): p. 3080-3093.

64. Rittirsch, D., Flierl, M.A. and Ward, P.A. Harmful molecular mechanisms in sepsis. Nature Reviews Immunology, 2008. 8: p. 776-787.

65. National vital Statistics report, 2009. 58.

66. Park, B.S., Song, D.H., Kim, H.M., Choi, B.S., Lee, H. and Lee, J.O. The structural basis of lipopolysacchride recognition by the TLR4-MD-2 Complex. Nature, 2009. 458: p. 1191-1196.

67. Cohen, J. The Immunopathogenesis of sepsis. Nature, 2002. 420: p. 885-891.

68. Raetz, C.R. and Whitfield, C. LIPOPOLYSACCHARIDE ENDOTOXINS. Annual review of Biochemistry, 2002. 71: p. 635-700.

69. Vaara, M. and Nikaido, H. Molecular basis of bacterial outer membrane permeability. Microbiological Reviews, 1985. 49: p. 1-32.

70. Barnickel, G., Bradaczek, H., Naumann, D., Rietschel, E.T., Giesbrecht, P. and Labischinski, H. High state of order of isolated bacterial lipopolysaccharide and its possible contribution to the permeation barrier property of the outer membrane. Journal of Bacteriology, 1985. 162: p. 9-13.

71. Hancock, R.E.W. Alterations in outer membrane permeability. Annual reviews of Microbiology, 1984. 38: p. 237-264.

72. Matsuura, M. Structural modifications of bacterial lipopolysaccharide that facilitate Gram negative bacteria evasion of host innate immunity. Frontiers in Immunology, $2013.4: 109$.

73. Karibian, D. and Caroff, M. Structure of bacterial lipopolysaccharide. Carbohydrate research, 2003. 338: p. 2431-2447. 
74. Snyder, S., Kim, D. and Mcintosh, T.J. Lipopolysaccharide bilayer structure: Effect of Chemotype, core mutations, divalent cations and temperature. Biochemistry, 1999. 38: p. 10758-10767.

75. Papo, N. and Shai, Y. A Molecular Mechanism for Lipopolysaccharide Protection of Gram-negative Bacteria from Antimicrobial Peptides*. Journal of Biological Chemistry, 2005. 280: p. 10378-10387.

76. Rosenfeld, Y., Sahl, H. and Shai, Y. Parameters involved in antimicrobial and endotoxin detoxification activities of antimicrobial peptides. Biochemistry, 2008. 47: p. 6468-6478.

77. Gutsmann, T.M., Mueller, M., Caroll, S.F., Mackenzie, R.C., Wiese, A. and Seydel, U. Dual role of lipopolysaccharide binding protein in neutralization of LPS and enhancement of LPS induced activation of mononuclear cells. Infection and Immunity, 2001. 69: p. 6942-6950.

78. Schromm, A.B., Brandenburg, K., Loppnow, H., Moran, A.P., Koch, M.H., Rietschel, E.T. and Sydel, U. Biological activities of lipopolysaccharides are determined by the shape of their lipidA portion. European Journal of Biochemistry, 2000. 267: p. 2008-2013.

79. Mueller, M., Brandenburg, K., Dedrick, R., Schromm, A.B. and Seydel, U. Phospholipids inhibit lipopolysaccharide(LPS) induced cell activation: A role for LPS-binding protein. The Journal of Immunology, 2011. 174: p. 1091-1096.

80. Brown, K.L. and Hancock, R.E.W. Cationic host defense (antimicrobial) peptides. Current Opinion in Immunology, 2006. 18: p. 24-30.

81. Marra, M.N., Wilde, C.G., Griffith, J.E., Snable, J.L. and Scott, R.W. Bactericidal/permeability increasing protein has endotoxin neutralizing ability. The Journal of Immunology, 1990. 144: p. 662-666.

82. Elsbach, P. The bactericidal/permeability increasing protein in antibacterial host defence. Journal of Leukocyte biology, 1998. 64: p. 14-18.

83. Beamer, L.J., Caroll, S.F. and Eisenberg, D. The BPI/LBP family of proteins: a structural analysis of conserved regions. Protein Science, 1998. 7: p. 906-914.

84. Tobias, P.S., Soldau, K., Iovine, N.M., Elsbach, P. and Weiss, J. Lipopolysaccharide binding proteins BPI and LBP form different types of 
complexes with LPS. The Journal of Biological Chemistry, 1997. 272: p. 1868218685.

85. Domingues, M.M., Castanho, M.A.R.B. and Santos, N.C. rBPI21 Promotes Lipopolysaccharide Aggregation and Exerts Its Antimicrobial Effects by (Hemi)fusion of PG-Containing Membranes. PLos One, 2009. 4(12): p. 8385-.

86. Velkov, T., Thompson, P.E., Nation, R.L. and Li, J. Structure-activity relationships of Polymyxin antibiotics. Journal of Medicinal Chemistry, 2010. 53: p. 1898-1916.

87. HsuChen, C.C. and Feingold, D.S. The mechanism of Polymyxin B action and selectivity towards biologic membranes. Biochemistry, 1973. 12: p. 2105-2111.

88. Stein, A. and Raoult, D. Colisitin: An antimicrobial for the 21st century. Clinical Infectious Diseases, 2002. 35: p. 901-902.

89. Srimal, S., Surolia, N., Balasubramanian, S. and Surolia, A. Titration calorimetric studies to elucidate the specificity of the interactions of polymyxin $B$ with lipopolysaccharides and lipidA. Biochemical Journal, 1996. 315: p. 679686.

90. Howe, J., Andra, J., Conde, R., Iriarte, M., Garidelm P., Koch, M.H.J., Gutsmann, T., Moriyon, I. and Brandenburg, K. Thermodynamic analysis of the lipopolysaccharide dependent resistance of Gram negative bacteria against polymyxin B. Biophysical Journal, 2007. 92: p. 2796-2805.

91. Pristovsek, P. and Kidric, J. Solution structure of Polymyxins B and E and effect of binding to lipopolysaccharide: An NMR and molecular modeling study. Journal of Medicinal Chemistry, 1999. 42: p. 4604-4613.

92. Mares, J., Kumaran, S., Gobbo,M. and Zerbe, O. Interactions of Lipopolysacchride and Polymyxin studied by NMR spectroscopy. Journal of Biological Chemistry, 2009. 284(17): p. 11498-11506.

93. Ried, C., Wahl, C., Miethke, T., Wellnhofer, G., Landgraf, C., Mergener, J.S. and Hoess, A. High affinity endotoxin-binidng and neutralizing peptides based on the crystal structure of recombinant Limulus anti-lipopolysaccharide factor. The Journal of Biological Chemistry, 1996. 271: p. 28120-28127. 
94. Hoess, A., Watson, S., Siber, G.R. and Liddington, R. Crysal structure of an endotoxin neutralizing protein from horseshoe crab, Limulus anti-LPS factor at 1.5A resolution. The EMBO Journal, 1993. 12: p. 3351-3356.

95. Pristovsek, P., Feher, K., Szilagyi, L. and Kidric, J. Structure of a synthetic fragment of the LALF protein when bound to lipopolysaccharide. Journal of Medicinal Chemistry, 2005. 48: p. 1666-1670.

96. Schuerholz, T., Brandenburg, K. and Marx, G. Antimicrobial peptides and their potential application in inflammation and sepsis. Critical Care, 2012. 16: p. 1-8.

97. Kowalski, I., Kaconis, Y., Andra, J., Olazaran, I.R., Gutsmann, T., Martinez de Tejada, G. and Brandenburg, K. Physicochemical and biological characterization of anti-endotoxin peptides and their influence on lipid properties. Protein and Peptide Letters, 2010. 17: p. 1328-1333.

98. Tomansinsig, L. and Zanetti, M. The Cathelicidins- structure, funtion and evolution. Current Protein and Peptide Science., 2005. 6: p. 23-34.

99. Uzzell, T., Stolzenberg, E.D., Shinnar, A.E. and Zasloff, M. Hagfish intestinal antimicrobial peptides are ancient cathelicidins. Peptides, 2003. 24: p. 16551667.

100. Saravanan, R. and Bhattacharjya, S. Oligomeric structure of a cathelicidin antimicrobial peptide in dodecylphosphocholine micelle determined by NMR spectroscopy. Biochimical et Biophysica Acta, 2010. 1808: p. 369-381.

101. Xiao, Y., Dai, H., Bommineni, Y.R., Soulages, J.L., Gong, Y.X., Prakash, O. and Zhang, G. Structure-activity relationships of fowlicidin-1. a cathelicidin antimicrobial peptide in chicken. FEBS journal, 2006. 273: p. 2581-2593.

102. Bhunia, A., Mohanram, H. and Bhattacharjya, S. Lipopolysacchride bound structures of the active fragments of Fowlicidin-1, a cathelicidin family of antimicrobial and antiendotoxic peptide from chicken, determined by transferred nuclear overhauser effect spectroscopy. Peptide Science, 2008. 92: p. 9-22.

103. Wang, G. Structures of human host defense Cathelicidin LL-37 and its smallest antimicrobial peptide KR-12 in lipid micelles. The Journal of Biological Chemistry, 2008. 283: p. 32637-32643. 
104. Tack, B.F., Sawai, M.V., Kearney, W.R., Robertson, A.D., Sherman, M.A., Wang, W., Hong, T., Boo, L.M., Wu,H., Waring, A.J. and Lehrer, R.I. SMAP-29 has two LPS-binding sites and a central hinge. European Journal of Biochemistry, 2002. 269: p. 1181-1189.

105. Nagaoka, I., Tamura, H. and Hirata,M. An antimicrobial cathelicidin peptide, Human CAP18/LL37, suppresses neutrophil apoptosis via the activation of formyl-peptide receptor like 1 and P2X7. The Journal of Immunology, 2006. 176: p. 3044-3052.

106. Durr, U.H.N., Sudheendra, U.S. and Ramamoorthy, A. LL37, the only human member of the cathelicidin family of antimicrobial peptides. Biochimica et Biophysica Acta, 2006. 1758: p. 1408-1425.

107. Skerlavaj, B., Benincasa, M., Risso, A., Zanetti, M. and Gennaro, R. SMAP-29: a potent antibacterial and antifungal peptide from sheep leucocytes. FEBS Letters, 1999. 463: p. 58-62.

108. Bhunia, A., Saravanan, R., Mohanram, H., Mangoni, M.L. and Bhattacharjya, S. NMR structures and interactions of Temporin-1Tl and Temporin-1Tb with Lipopolysaccharide micelles. Mechanistic insights into outer membrane permeabilization and synergisitc activity. The Journal of biological chemistry, 2011. 286: p. 24394-24406.

109. Rosenfeld, Y, Barra, D., Simmaco, M, Mangoni, M.L. and Shai, Y. A synergism between Temporins towards gram negative bacteria overcomes resistance imposed by the lipopolysaccharide protective layer. Journal of Biological Chemistry, 2006. 281(39): p. 28565-28574.

110. Brogden, N.K. and Brogden, K.A. Will new generations of modified antimicrobial peptides improve their potential as pharmaceuticals? International Journal of Antimicrobial Agents, 2011. 38: p. 217-225.

111. Aoki, W. and Ueda, M. Characterisation of Antimicrobial peptides toward the development of Novel antibiotics. Pharmaceuticals, 2013. 6: p. 1055-1081.

112. Fjell, C.D., Hiss, J.A., Hancock, R.E.W. and Schneider, G. Designing antimicrobial peptides: form follows function. Nature reviews Drug Discovery, 2012. 11: p. 37-51. 
113. Rosenfeld, Y., Lev, N. and Shai, Y. Effect of the Hydrophobicity to Net Positive Charge Ratio on Antibacterial and Anti-Endotoxin Activities of Structurally Similar Antimicrobial Peptides. Biochemistry, 2010. 49: p. 853-861.

114. Shai, Y. From Innate Immunity to de-novo designed Anti-microbial peptides. Current Pharmaceutical design, 2002. 8: p. 715-725.

115. Yu, H.Y., Yip, B.S., Tu, C.H., Chen, H.L., Chu, H.L., Chih, Y.H., Cheng, H.T., Sue, S.C. and Cheng, J.W. Correlations between membrane immersion depth, orientation and salt resistance of tryptophan rich antimicrobial peptides. Biochemica et Biophysica Acta, 2013. 1828: p. 2720-2728.

116. Boman, H.G., Wade, D., Boman, I.A., Wahlin, B. and Merrifield, R.B. Antibacterial and antimalarial properties of peptides that are cecropin-melittin hybrids. FEBS Letters, 1989. 259: p. 103-106.

117. Pasupleti, M., Schmidtchen, A., Chalupka, A., Ringstad, L. and Malmsten, M. End-Tagging of ultra short antimicrobial peptides by W/F stretches to facilitate bacterial killing. PLos One, 2009.

118. Serrano, G.N., Zhanel, G.G. and Schweizer, F Antibacterial activity of ultrashort cationic lipo-beta peptides. Antimicrobial agents and chemotherapy, 2009. 53: p. 2215-2217.

119. Findlay, B., Zhanel, G.G. and Schweizer, F. Cationic amphiphiles, a new generation of antimicrobials inspired by the natural antimicrobial peptide scaffold. Antimicrobial agents and chemotherapy, 2010. 54(4049-4058).

120. Andra, J., Lohner, K., Blondelle, S.E., Jerala, R., Moriyon, I., Koch, M.H., Garidel,P. and Brandenburg, K. Enhancement of endotoxin neutralization by coupling of a C12-alkyl chain to a lactoferricin derived peptide. Biochemical Journal, 2005. 385: p. 135-143.

121. Majerle,A., Kidric, J. and Jerala, R. Enhancement of antibacterial and lipopolysacchride binding activities of a human lactoferrin peptide fragment by the addition of acyl chain. Journal of antimicrobial chemotherapy, 2003. 51: p. 1159-1165.

122. Zwytick, D., Pabst, G., Abuja, P.M., Jilek, A., Blondelle, S.E., Andra, J., Jerala, R., Monreal, D., Martinez de Tejada, G. and Lohner, K. Influence of N-acylation 
of a peptide derived from human lactoferricin on membrane selectivity. Biochimica et Biophysica Acta, 2006. 1758: p. 1426-1435.

123. Mangoni, M.L., Epand, R.F., Rosenfeld, Y., Peleg, A., Barra, D., Epand, R.M. and Shai, Y. Lipopolysaccharide, a Key Molecule Involved in the Synergism between Temporins in Inhibiting Bacterial Growth and in Endotoxin Neutralization. Journal of Biological Chemistry, 2008. 283: p. 22907-22917.

124. Lockwood, N.A., Haseman, J.R., Tirrell, M.V. and Mayo, K.H. Acylation of SC4 dodecapeptide increases bactericidal potency against Gram positive bacteria, including drug resistant strains. Biochemical Journal, 2004. 378: p. 93-103.

125. Etzerodt, T., Henriksen, J.R., Rasmussen, P., Clausen, M.H. and Andresen, T.L. Selective acylation enhances membrane charge sensitivity of the antimicrobial peptide Mastoporan-X. Biophysical Journal, 2011. 100: p. 399-409.

126. Moellering, R.C.J. Antimicrobial Synergism: An elusive concept. The Journal of Infectious diseases, 1979. 140: p. 639-641.

127. Westerhoff, H.V., Zasloff, M., Rosner, J.L., Hendler, R.W., de Waal, A., VazGomes, A., Jongsma, P.M., Riethorst, A. and Juretic, D. Functional synergism of the magainins PGLa and magainin-2 in Escherichia coli, tumor cells and liposomes. European Journal of Biochemistry, 1995. 228: p. 257-264.

128. Strandberg, E., Tremouilhac, P., Wadhwanti, P. and Ulrich, A.S. Synergistic transmembrane insertion of the heterodimeric PGLa/magainin 2 complex studied by solid-state NMR. Biochimica et Biophysica Acta, 2009. 1788: p. 1667-1679.

129. Shin, S.Y., Kang, J.H. and Hahm, K.S. Structure-antibacterial, antitumor and hemolytic activity relationships of cecropin A-magainin 2 and cecropin Amelittin hybrid peptides. Journal of Peptide Research, 1999. 53: p. 82-90.

130. Ferre, R., Melo, M.N., Correia, A.D., Feliu, L., Bardaji, E. and Planas, M. Synergistic effects of the membrane actions of Cecropin-Melittin antimicrobial hybrid peptide BP100. Biophysical Journal, 2009. 96: p. 1815-1827.

131. Torcato, I.M., Huang, Y.H., Franquelim, H.G., Gaspar, D., Craik, D.J., Castanho, M.A.R.B. and Henriques, S.T. Design and characterisation of novel antimicrobial peptides, R-BP100 and RW-BP100, with activity against Gram 
negative and Gram positive bacteria. Biochimica et Biophysica Acta, 2013. 1828: p. 944-955.

132. Dhople, V. and A. Krukemeyer, Ramamoorthy, A., The human beta-defensin-3, an antibacterial peptide with multiple biological funtions. Biochimica et Biophysica Acta, 2006. 1758: p. 1499-1512.

133. Jung, S., Mysliwy, J., Spudy, B., Lorenzen, I., Reiss, K., Gelhaus,C., Podschun, R., Leippe, M. and Grotzinger, J. Human beta defensin 2 and beta defensin 3 chimeric peptides reveal the structural basis of the pathogen specificity of their parent molecules. Antimicrobial agents and chemotherapy, 2011. 55: p. 954960.

134. Dathe, M., Nikolenko, H., Klose, J. and Bienert,M. Cyclization increases the antimicrobial activity and selectivity of arginine and tyrptophan containing hexa peptides. Biochemistry, 2004. 43: p. 9140-9150.

135. Unger, T. , Oren, Z. and Shai, Y. The effect of cyclization of Magainin2 and Melittin analogues on structure, funtion and model membrane interactions: Implications to their mode of action. Biochemistry, 2001. 40: p. 6388-6397.

136. Gobbo, M., Biondi, L., Filira, F., Gennaro, R., Benincasa, M., Scolaro, B. and Rocchi, R. Antimicrobial peptides: Synthesis and antibacterial activity of linear and cyclic Drosocin and Apidaecin $1 b$ analogues. Journal of Medicinal Chemistry, 2002. 45: p. 4494-4504.

137. Ramamoorthy, A., Thennarasu, S., Tan, A., Gottipati, K., Sreekumar, S., Hey, D.L., An, F.Y.P., and Shelburne, C.E. Deletion of all cysteines in tachyplesin-1 abolishes hemolytic activity and retains antimicrobial activity and lipopolysaccharide selective binding. Biochemistry, 2006. 45: p. 6529-6540.

138. Varkey, J. and Nagaraj, R. Antibacterial activity of human neutrophil defensin HNP-1 analogs without cysteines. Antimicrobial agents and chemotherapy, 2005. 49: p. 4561-4566.

139. Schmidtchen, A., Pasupuleti, M., Morgelin, M., Davoudi, M., Alenfall, J., Chalupka, A. and Malmsten, M. Boosting antimicrobial peptides by hydrophobic oligopeptide end tags. The Journal of Biological Chemistry, 2009. 284: p. 17584-17594. 
140. Pasupleti, M., Schmidtchen, A., Chalupka, A., Ringstad, L. and Malmsten, M. End-Tagging of ultra short antimicrobial peptides by W/F stretches to facilitate bacterial killing. PLos One, 2009.

141. Serrano, G.N., Zhanel, G.G. and Schwiezer, F. Antibacterial activity of ultrashort cationic lipo beta peptides. Antimicrobial agents and chemotherapy, 2009. 53: p. 2215-2217.

142. Yu, H.Y., Tu, C.H., Yip, B.S., Chen, H.L., Cheng, H.T., Huang, K.C., Lo, H.J., Cheng, J.W. Easy strategy to incrase salt resistance of antimicrobial peptides. Antimicrobial agents and chemotherapy, 2011. 55: p. 4918-4921.

143. Haney, E.F. and Vogel, H.J. NMR of antimicrobial peptides. Annual reports on NMR spectroscopy, 2009. 65: p. 1-51.

144. Haney, E.F., Hunter, H.N., Matsuzaki, K., Vogel, H.J. Solution NMR studies of amphibian antimicrobial peptides: Linking structure to function? Biochimica et Biophysica Acta 2009. 1788: p. 1639-1655.

145. Japelj, B., Pristovsek, P., Majerle, A. and Jerala, R. Structural origin of endotoxin neutralization and antimicrobial activity of a Lactoferrin-based peptide. The Journal of Biological Chemistry, 2005. 280: p. 16955-16961.

146. Domadia, P.N., Bhunia, A., Ramamoorthy, A. and Bhattacharjya, S. Structure, Interactions, and Antibacterial Activities of MSI-594 Derived Mutant Peptide MSI-594F5A in Lipopolysaccharide Micelles: Role of the Helical Hairpin Conformation in outer-Membrane permeabilization. Journal of American Chemical Society, 2010. 132: p. 18417-18428.

147. Gottler, L.M. and Ramamoorthy, A. Structure, membrane orientation, mechanism and funtion of Pexiganan-A highly potent antimicrobial peptide designed from magainin. Biochimica et Biophysica Acta, 2009. 1788: p. 16801686.

148. Ferguson, A.D., Welte, W., Hofmann, E., Lindner, B., Holst, O., Coulton, J.W. and Diederichs, K. A conserved structural motif for lipopolysaccharide recognition by procaryotic and eucaryotic proteins. Structure, 2000. 8: p. 585592. 
149. Bhattacharjya, S., Domadia, P.N., Bhunia, A., Malladi, S. and David, S. HighResolution Solution Structure of a Designed Peptide Bound to Lipopolysaccharide: Transferred Nuclear Overhauser Effects, Micelle Selectivity, and Anti-Endotoxic Activity. Biochemistry, 2007. 46: p. 5864-5874.

150. Bhunia, A., Mohanram, H., Domadia, P.N., Torres, J. and Bhattacharjya, S. Designed Beta-Boomerang Antiendotoxic and Antimicrobial Peptides :STRUCTURES AND ACTIVITIES IN LIPOPOLYSACCHARIDE. Journal of Biological Chemistry, 2009. 284: p. 21991-22004.

151. Tam, J.P., Wu, C.R., Liu, W. and Zhang, J.W. Disulfide bond formation in peptides by Dimethyl sulfoxide. Scope and applications. Journal of American Chemical Society, 1991. 113: p. 6657-6662.

152. Avrahami, D. and Shai, Y. Conjugation of a Magainin Analogue with Lipophilic Acids Controls Hydrophobicity, Solution Assembly, and Cell Selectivity. Biochemistry, 2002. 41: p. 2254-2263.

153. Tsubery, H., Ofek, I., Cohen, S. and Fridkin, M. N-terminal modifications of Polymyxin B nonapeptide and their effect on antibacterial activity. Peptides, 2001. 22: p. 1675-1681.

154. Japelj, B., Zorko, M., Marjerle, A., Pristovek, P., Sanchez-Gomez, S., Matinez de Tejada, G., Moriyon, I., Blondelle, S.E., Brandenberg, K., Andra, J., Lohner, $\mathrm{K}$. and Jerala, R. The acyl group as the central element of the structural organization of antimicrobial lipopeptide. Journal of American Chemical Society, 2007. 129: p. 1022-1023.

155. Liu, L.P. and Deber, C.M. Guidelines for Membrane protein engineering derived from De novo designed model peptides. Biopolymers (Peptide Science), 1998. 47: 41.

156. Makovitzki, A., Avrahami, D. and Shai, Y. Ultrashort antibacterial and antifungal lipopeptides. Proceedings of National Academy of Sciences, 2006. 103(43): p. 15997-16002.

157. Shalev, D.E., Rotem, S., Fish, A. and Mor, A. Consequences of N-Acylation on structure and Membrane binding properties of Dermaseptin derivative K4-S4(1-13). Journal of Biological Chemistry, 2006. 281(14): p. 9432-9438. 
158. Haney, E.F. and Vogel, H.J. NMR of antimicrobial peptides. Annual reports on NMR spectroscopy, 2009. 65.

159. Fujitani, N., Kouno, T., Nakahara, T., Takaya, K., Osaki, T., Kawabata, S.I., Mizuguchi, M., Aizawa, T., Demura, M., Nishimura, S.I. and Kawano, K. The solution structure of horseshoe crab antimicrobial peptide tachystatinB with an inihibitory cystine-knot motif. Journal of Peptide science, 2007. 13: p. 269-279.

160. Zweytick, D., Deutsch, G., Andra, J., Blondelle, S.E., Vollmer, E., Jerala, R. and Lohner, K. Studies on Lactoferricin-derived Escherichia coli membrane active peptides reveal differences in the mechanism of $\mathrm{N}$-acylated versus Nonacylated peptides. Journal of Biological Chemistry, 2011. 286(24): p. 21266-21276.

161. Rosenfeld, Y, Barra, D., Simmaco, M, Mangoni, M.L. and Shai, Y. A synergism between Temporins towards gram negative bacteria overcomes resistance imposed by the lipopolysaccharide protective layer. Journal of Biological Chemistry, 2006. 281(39): p. 28565-28574.

162. Papo, N. and Shai, Y. A moleuclar mechanism for lipopolysaccharide protection of Gram negative bacteria from antimicrobial peptides. The Journal of Biolgical Chemistry, 2005. 280: p. 10378-10387.

163. Abbassi, F., Lequin, O., Piesse,C., Goasdoue,N., Foulon,T., Nicolas,P. and Ladram,A. Temporin-SHf, a new type of Phe-rich and hydrophobic ultrashort antimicrobial peptide. The Journal of Biolgical Chemistry, 2010. 285: 16880.

164. Li, X., Li, Y., Han, H., Miller, D.W. and Wang, G. Solution structures of Human LL-37 fragments and NMR based identification of a minimal membrane targeting Antimicrobial and Anticancer region. Journal of American chemical society, 2006. 128.

165. Grieco, P., Luca, V., Auriemma, L., Carotenuto, A., Saviello, M.R., Campiglia, P., Barra, D., Novellinoa, E. and Mangoni, M.L. Alanine scanning analysis and structure-function relationships of the frog-skin antimicrobial peptide temporin1Ta. Journal of Peptide Science, 2011. 17: p. 358-365.

166. Makovitzki, A., Avrahami, D. and Shai, Y. Ultrashort antibacterial and antifungal lipopeptides. Proceedings of National Academy of Sciences, 2006. 103(43): p. 15997-16002. 
167. Sims, P.J., Waggoner, A.S., Wang, C.H. and Hoffman, J.F. Studies on the mechanism by which cyanine dyes measure membrane potential in Red blood cells and Phosphatidylcholine vesicles. Biochemistry, 1974. 13(16): p. 33153330.

168. Chan, D.I., Prenner, E.J. and Vogel, H.J. Tryptophan and arginine rich antimicrobial peptides: Structures and mechanisms of action. Biochimica et Biophysica Acta, 2006. 1758: p. 1184-1202.

169. David S.Wishart, C.G.B., Arne Holm, Robert S.Hodges, Brian D.Sykes, 1H,13C and $15 N$ random coil NMR chemical shifts of the common aminoacids. Investigations of nearest-neighbor effects. Journal of Biomolecular NMR, 1995. 5: p. 67-81.

170. Berjanskii, M.V., Neal, S. and Wishart, D.S. PREDITOR: a web server for predicting protein torsion angle constraints. Nucleic acids research, Web server issue, 2006. 34: p. W63-69.

171. Laskowski, R.A., Rullmann, J.A., MacArthur, M.W., Kaptein, R. and Thornton, J.M. AQUA and PROCHECK NMR: programs for checking the quality of protein structures solved by NMR. Journal of Biomolecular NMR, 1996. 8: $\mathrm{p}$. 477-496.

172. Ramamoorthy, A. and Bhattacharjya, S Multifunctional host defense peptides: functional and mechanistic insights from NMR structures of potent antimicrobial peptides. The FEBS journal, 2009. 276: p. 6465-6473.

173. Mishra, B. and Wang, G. Ab initio design of potent anti-MRSA peptides based on batabase filtering technology. Journal of American Chemical Society, 2012. 134: p. 12426-12429.

174. Kokryakov, V.N., Harwig, S.S.L., Panyutich, E.A., Shevchenko, A.A., Aleshina, G.M., Shamova, O.V., Korneva, H.A. and Lehrer, R.I. Protegrins: leukocyte antimicrobial peptides that combine features of corticostatic defensins and tachyplesins. FEBS, 1993. 327: p. 231-236.

175. Fahrner, R.L., Dieckmann, T., Harwig, S.S.L., Lehrer, R.I., Eisenberg, D. and Feigon, J. Solution structure of protegrin-1, a broad spectrum antimicrobial peptide from porcine leukocytes. Chemistry and Biology, 1996. 3: p. 543-550. 
176. Langham, A.A., Khandelia, H. and Kaznessis, Y.N. how can a beta sheet peptide be both a potent antimicrobial and harmfully toxic? Molecular dynamics simulations of Protegrin-1 in micelles. Biopolymers (Peptide Science), 2005. 84: p. 219-231.

177. Bolintineanu, D.S. and Kaznessis, Y.N. Computational studies of protegrin antimicrobial peptides: a review. Peptides, 2011. 32: p. 188-201.

178. Steinberg, D.A., Hurst, M.A., Fujii,C.A., Kung,A.H., Ho,J.F., Cheng,F.C., Loury,D.J. and Fiddes, J.C., Protegrin-1:a broad spectrum, rapidly microbicidal peptide with invivo activity. Antimicrobial agents and chemotherapy, 1997. 41: p. 1738-1742.

179. Koradi, R., Billeter, M. and Wuthrich, K. MOLMOL: a program for display and analysis of macromolecular structures. Journal of molecular graphics, 1996. 14: p. 51-55.

180. Mani, R., Waring, A.J., Lehrer, R.I. and Hong, M. Membrane disruptive abilities of beta hairpin antimicrobial peptides correlate with conformation and activity: A 31P and 1H NMR study. Biochimica et Biophysica Acta, 2005. 1716: p. 1118.

181. Thomas, A., Meurisse, R., Charloteaux, B. and Brasseur, R. Aromatic side-chain interactions in proteins. I.Main structural features. Proteins-structure,Funtion and Genetics, 2002. 48: p. 628-634.

182. Friedrich, C., Scott, G.M., Karunaratne, N.,Yan,H. and Hancock R.E.W. Saltresistant alpha helical cationic antimicrobial peptides. Antimicrobial agents and chemotherapy, 1999. 43: p. 1542-1548.

183. Lee, J.Y., Tang, S.T., Lee, S.K., Jung, H.H., Shin, S.Y., Hahm, K.S. and Kim, J.I. Salt-resistant homodimeric bactenecin, a cathelicidin derived antimicrobial peptide. The FEBS journal, 2008. 275: p. 3911-3920.

184. Lai, J.R., Huck, B., Weisblum, B. and Gellman, S.H. Design of non cysteine containing antimicrobial beta hairpins: Structure-activity relationship studies wiht linear Protegin-1 analogues. Biochemistry, 2002. 41: p. 12835-12842. 
185. Li, X., Saravanan, R., Kwak, S.K., Leong, S.S.J. Biomolecular engineering of a human beta defensin model for increased salt resistance. Chemical Engineering Science, 2013. 95: p. 128-137.

186. Nityakalyani Srinivas, P.J., Bernhard J. Ueberbacher, Martina Werneburg, Katja Zerbe, Jessica Steinmann, Benjamin Van der Meijden, Francesca Bernardini, Alexander Lederer, Ricardo L. A. Dias, Pauline E. Misson, Heiko Henze, Ju rg Zumbrunn, Frank O. Gombert, Daniel Obrecht, Peter Hunziker, Stefan Schauer, Urs Ziegler, Andres Ka ch, Leo Eberl, Kathrin Riedel, Steven J. DeMarco, John A. Robinson, Peptidomimetic antibiotics target outer-membrane biogenesis in Pseudomonas aeruginosa. Science, 2010. 327: p. 1010-1013.

187. Saravanan, R., Xi, L., Lim, K., Mohanram, H., Peng, L., Mishra, B., Basu, A., Lee, J.M., Bhattacharjya, S. and Leong, S.S.J. Design of short membrane selective antimicorbial peptides containing tryptophan and arginine residues for improved activity, salt resistance and biocompatibility. Biotechnology and Bioengineering, 2013. 111: p. 37-49.

188. Goldman, M.J., Anderson, M.G., Stolzenberg, E.D., Prasadkari, U., Zasloff, M. and Wilson, M.J. Human beta defensin-1 is a salt sensitive antibiotic in lung that is inactivated in cystic fibrosis. Cell, 1997. 88: p. 553-560.

189. HaiNan, Y. and Shai, Y. Effect of disulphide bond position on salt resistance and LPS neutralizing activity of alpha helical homodimeric model antimicrobial peptides. BMB reports, 2011. 44: p. 747-752.

190. Park, I.Y., Cho, J.H., Kim, K.S., Kim, Y.B., Kim, M.S. and Kim, S.C. Helix stability confers salt resistance upon helical antimicrobial peptides. The Journal of Biological Chemistry, 2004. 279: p. 13896-13901.

191. Talbot, G.H., Bradley, J., Edwards, J.E., Gilbert, D., Scheld, M. and Bartlett, J.G., Bad bugs need drugs: An update on the development pipeline form the antimicrobial availability task force of the infectious diseases society of America. Clinical infectious diseases, 2006. 42: p. 657-668. 